\title{
Microstructural Examination to Aid in Understanding Friction Bonding Fabrication Technique for Monolithic Nuclear Fuel
}

Karen L. Shropshire

April 2008

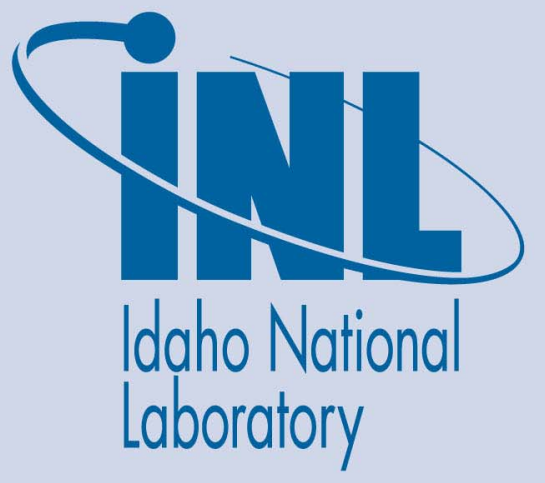

The INL is a U.S. Department of Energy National Laboratory operated by Battelle Energy Alliance 


\title{
Microstructural Examination to Aid in Understanding Friction Bonding Fabrication Technique for Monolithic Nuclear Fuel
}

\author{
Karen L. Shropshire
}

April 2008

\begin{abstract}
Idaho National Laboratory
Idaho Falls, Idaho 83415
\end{abstract}





\title{
MICROSTRUCTURAL EXAMINATION TO AID IN UNDERSTANDING FRICTION BONDING FABRICATION TECHNIQUE \\ FOR MONOLITHIC NUCLEAR FUEL
}

by

Karen L. Shropshire

\begin{abstract}
A thesis
submitted in partial fulfillment

of the requirements for the degree of

Master of Science in the Department of Nuclear Science and Engineering
\end{abstract}

Idaho State University

April 2008 


\section{DISCLAIMER}

This information was prepared as an account of work sponsored by an agency of the U.S. Government. Neither the U.S. Government nor any agency thereof, nor any of their employees, makes any warranty, express or implied, or assumes any legal liability or responsibility for the accuracy, completeness, or usefulness of any information, apparatus, product, or process disclosed, or represents that its use would not infringe privately owned rights. References herein to any specific commercial product, process, or service by trade name, trademark, manufacturer, or otherwise, does not necessarily constitute or imply its endorsement, recommendation, or favoring by the U.S. Government or any agency thereof. The views and opinions of authors expressed herein do not necessarily state or reflect those of the U.S. Government or any agency thereof. 


\section{ACKNOWLEDGEMENTS}

The author gratefully acknowledges this work's funding, supplied by the Department of Energy's Global Threat Reduction Initiative, through the Reduced Enrichment for Research and Test Reactors program at the Idaho National Laboratory; appreciation to Karen Moore for helping to open the door for my participation in this research opportunity; thank you to Dr. Jan-Fong Jue, for his patient training on sample preparation and examination techniques; gratitude to Dr. Douglas Burkes for teaching me about monolithic fuel and the friction bonding process, and his guidance and consultation on my research and results; and appreciation to my graduate advisor in the Idaho State University College of Engineering, Dr. Mary Lou DunzikGougar, for her flexibility and staunch support of my graduate studies.

Any opinions contained in this document are solely the author's and do not represent the opinions or positions of the Department of Energy, Battelle Energy Alliance, Idaho National Laboratory, or Idaho State University. 


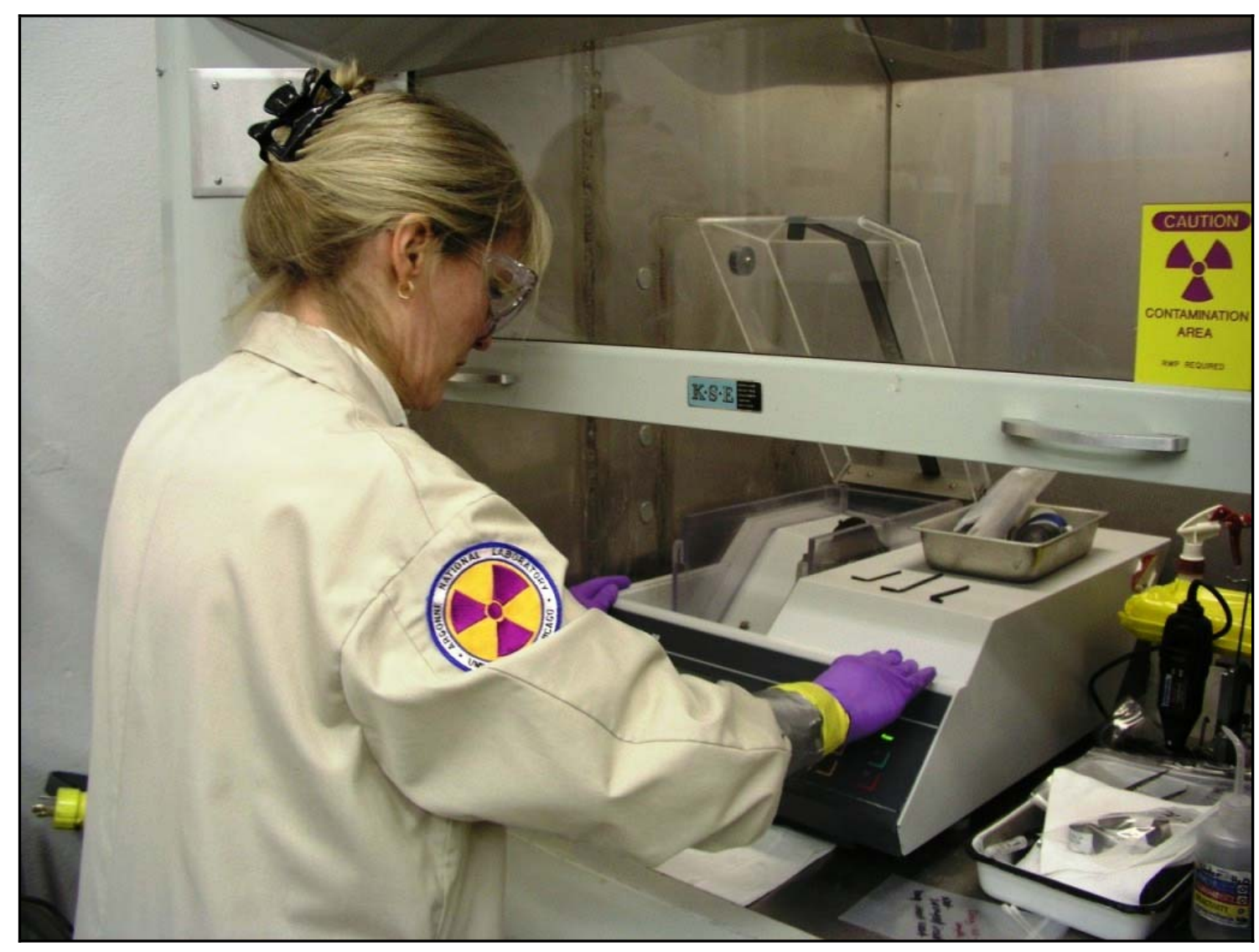




\begin{abstract}
$\underline{\text { Abstract }}$
Monolithic nuclear fuel is currently being developed for use in research reactors, and friction bonding (FB) is a technique being developed to help in this fuel's fabrication. Since both FB and monolithic fuel are new concepts, research is needed to understand the impact of varying FB fabrication parameters on fuel plate characteristics. This thesis research provides insight into the FB process and its application to the monolithic fuel design by recognizing and understanding the microstructural effects of varying fabrication parameters (a) FB tool load, and (b) FB tool face alloy. These two fabrication parameters help drive material temperature during fabrication, and thus the material properties, bond strength, and possible formation of interface reaction layers. This study analyzed temperatures and tool loads measured during those FB processes and examined microstructural characteristics of materials and bonds in samples taken from the resulting fuel plates.
\end{abstract}

This study shows that higher tool load increases aluminum plasticization and forging during FB, and that the tool face alloy helps determine the tool's heat extraction efficacy. The study concludes that successful aluminum bonds can be attained in fuel plates using a wide range of FB tool loads. The range of tool loads yielding successful uranium-aluminum bonding was not established, but it was demonstrated that such bonding can be attained with FB tool load of 48,900 N (11,000 lbf) when using a FB tool faced with a tungsten alloy. This tool successfully performed FB, and with better results than tools faced with other materials.

Results of this study correlate well with results reported for similar aluminum bonding techniques. This study's results also provide support and validation for other nuclear fuel development studies and conclusions. Recommendations are offered for further research. 


\section{TABLE OF CONTENTS}

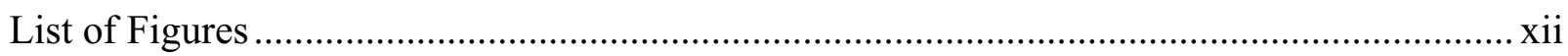

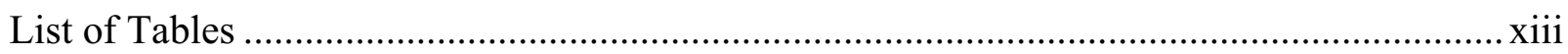

List of Acronyms and Abbreviations ..................................................................................

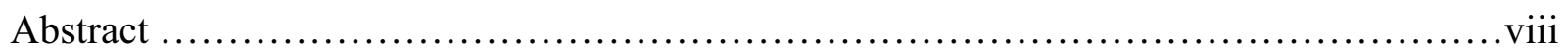

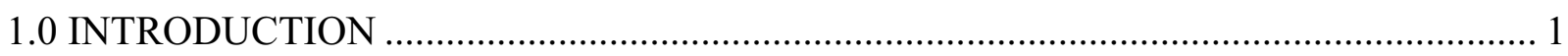

1.1 Reduced Enrichment for Research and Test Reactors (RERTR) Program Mission........ 1

1.2 Monolithic Fuel Design ................................................................................... 3

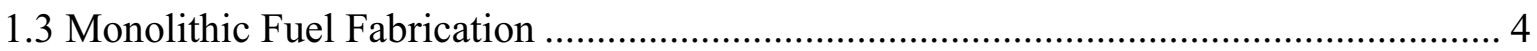

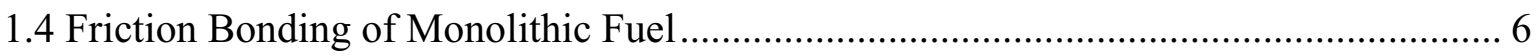

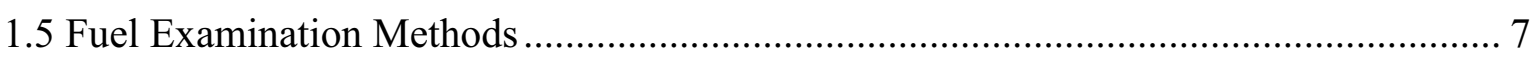

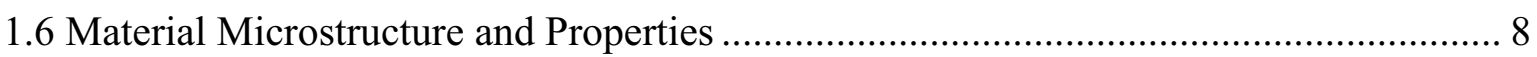

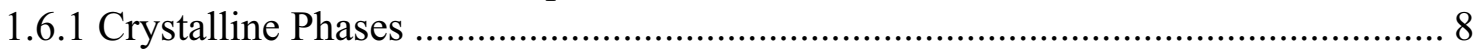

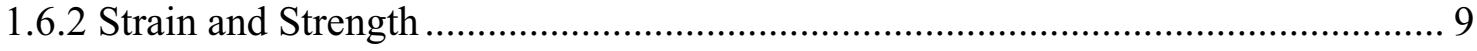

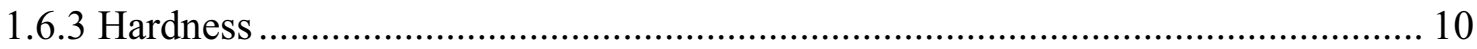

1.6.4 Temperature Effects ……………................................................................. 11

2.0 LITERATURE SEARCH: STATE OF THE ART IN FRICTION BONDING AND ITS APPLICATION TO FUEL PLATE FABRICATION .................................................... 13

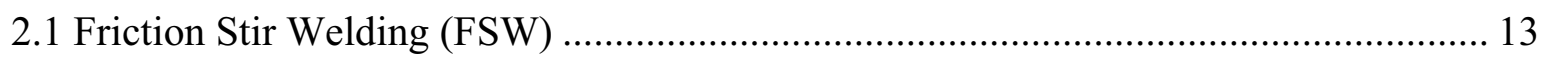

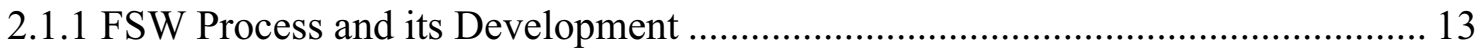

2.1.2 Microstructure after Friction Stir Welding....................................................... 16

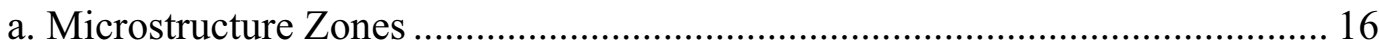

b. Grain Sizes, Shapes, and Directions …………………................................. 17

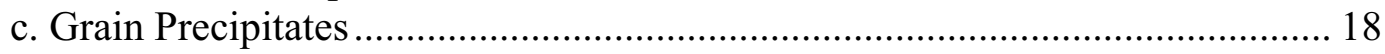

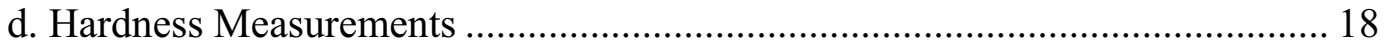

e. Strength and Formability......................................................................... 19

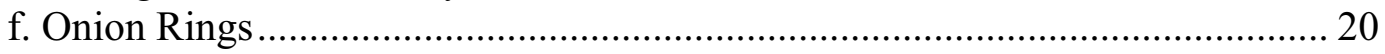

2.1.3 Effects of Fabrication Parameters ....................................................................... 21

a. Complex Process Mechanisms During Friction Stir Welding …………............ 21

b. Fabrication Parameters' Effects on Microstructure ............................................ 22

c. Fabrication Parameters' Effects on Macrostructure........................................... 24

2.2 RERTR Program's Monolithic Fuel and FB Fabrication .......................................... 24

2.2.1 Overview of RERTR Fuel Development Program.......................................... 24

a. RERTR Program's Irradiation Experiments to Date ...................................... 26

b. RERTR Program's Plans for Future Irradiation Experiments ........................... 27

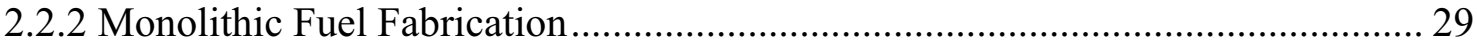

a. Foil Production to Prepare for Monolithic Fuel Fabrication.............................. 29

b. Fuel Fabrication by Transient Liquid Phase Bonding (TLPB) ......................... 30

c. Fuel Fabrication by Hot Isostatic Pressing (HIP) ............................................. 31

d. Fuel Fabrication by Friction Bonding (FB) ............................................... 31

2.2.3 Monolithic Fuel Examination Findings.............................................................. 37

a. Findings of Irradiation-Performance Evaluations of Monolithic Fuel.............. 37

b. Findings of Pre-Irradiation Examinations of Monolithic Fuel .......................... 39 


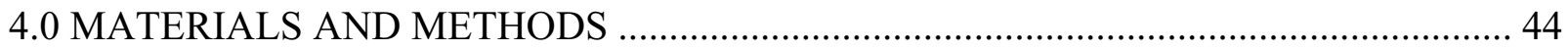

4.1 General Plate Fabrication and Sample Preparation ............................................. 44

4.2 Friction Bonding, Preparation and Examination of Aluminum Samples Fabricated with

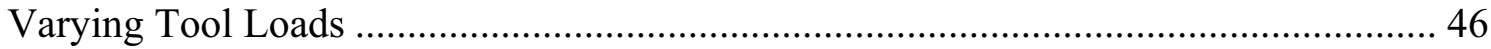

4.3 Friction Bonding, Preparation, and Examination of DU10Mo Samples Fabricated with

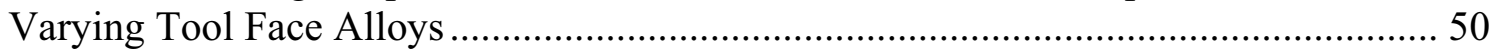

4.3.1 General Friction Bonding Process on DU10Mo Fuel Plate Sets ....................... 50

4.3.2 Samples from Specific Locations within DU10Mo Fuel Plates ......................... 56

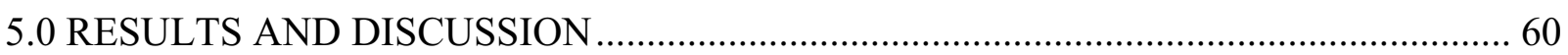

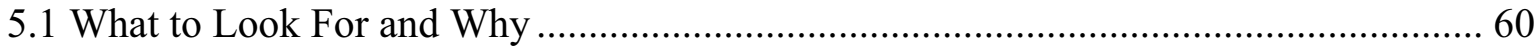

5.2 Results and Discussion of Examination of Aluminum Samples Fabricated with Varying

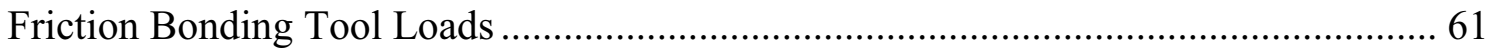

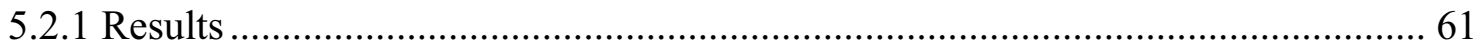

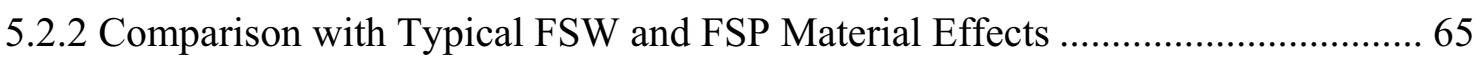

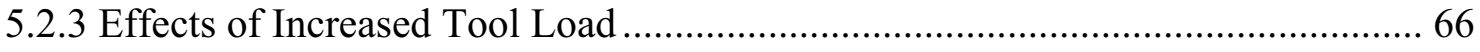

5.2.4 Microstructure Differences depending on Location in Sample .......................... 67

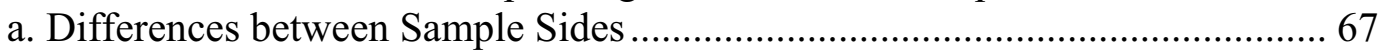

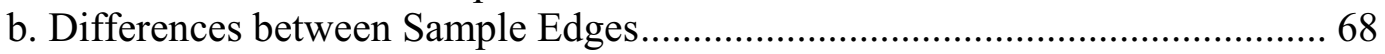

5.3 Results and Discussion of Friction Bonding and Examination of DU10Mo Samples

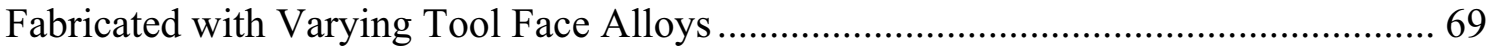

5.3.1 Analysis of General Friction Bonding Process on DU10Mo Fuel Plate Sets ...... 69

a. Heat Removal Efficiency of Tool Face Alloys ............................................. 70

b. Load and Temperature Trends during the Friction Bonding Process ............... 72

5.3.2 Analysis of Samples from Specific Locations within DU10Mo Fuel Plates ....... 73

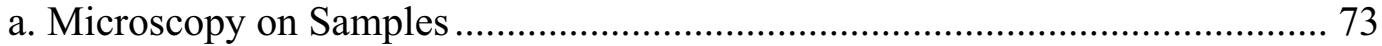

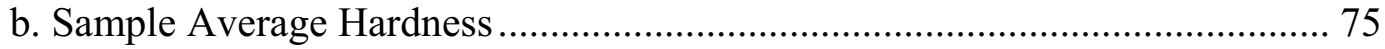

c. Location-Specific Tool Temperatures and Sample Hardness .......................... 78

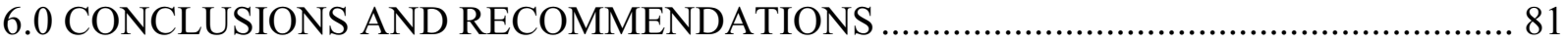

6.1 Conclusions from this Thesis Study ............................................................... 81

6.1.1 Conclusions from Examination of Aluminum Samples Fabricated with Varying

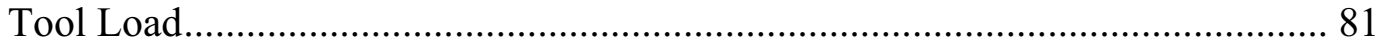

6.1.2 Conclusions from Friction Bonding and Examination of DU10Mo Samples

Fabricated with Varying Tool Face Alloys................................................... 81

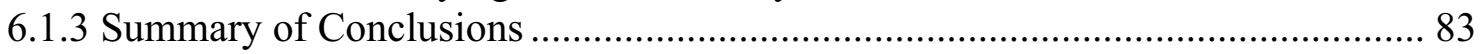

a. Conclusions Regarding Tool Load ............................................................. 83

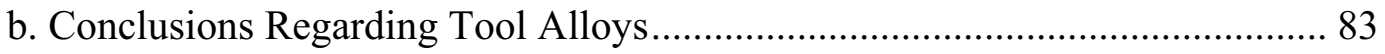

c. Conclusions Regarding the Friction Bonding Process.................................. 83

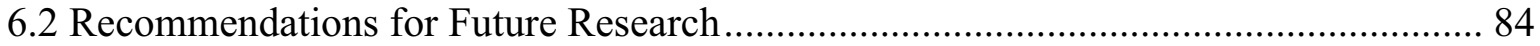

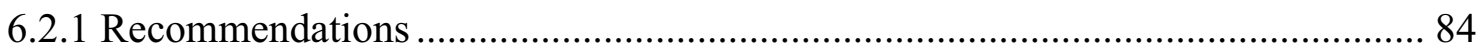

6.2.2 Summary of Recommendations ............................................................ 85

a. Further Examination of These Samples and Fuel Plates............................... 85

b. Research on Friction Bonding Temperatures .......................................... 85

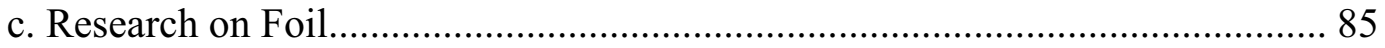


APPENDIX A - Literature Search on RERTR Fuel Material Phenomena and Challenges

A.1 Introduction

A.2 Uranium-Molybdenum Material Phases

A.3 Interface Reaction Layers

A.3.1 Reaction Layer Formation and Effects

A.3.2 Reaction Layer Formed During Fabrication of Monolithic Fuel

A.3.3 Reaction Layer Formed During Irradiation of Monolithic Fuel

A.4 References

APPENDIX B - Photos of Fuel Plate Fabrication, Sample Preparation, and Examination Equipment

B-1 FASB Rolling Machine

B-2 Current Friction Bonding Machine at FASB

B-3 New Friction Bonding Machine at FASB

B-4 Aluminum Plates after Friction Bonding

B-5 FASB Light Table

B-6 Shear in FASB West Radiation Hood

B-7 FASB High-Speed Saw

B-8 Sample Mounting Epoxy Kit

B-9 EML Sample Polishing Stations

B-10 EML Sample Etching Stations

B-11 EML Computer, Optical Microscopes, and Microhardness Tester

APPENDIX C - Microphotos of One-Sided, Single-Pass Friction Bonded Aluminum Samples

C-1 Microphotos of Sample Fabricated with $35.6 \mathrm{kN}$ (8,000 lbf) Target FB Tool Load

C-2 Microphotos of Sample Fabricated with $44.5 \mathrm{kN}(10,000 \mathrm{lbf})$ Target FB Tool Load

C-3 Microphotos of Sample Fabricated with 53.4 kN (12,000 lbf) Target FB Tool Load

C-4 Microphotos of Sample Fabricated with 62.3 kN (14,000 lbf) Target FB Tool Load

APPENDIX D - Photos of DU10Mo Monolithic Fuel Plate Samples

D-1 Microphotos of Sample Fabricated with FB Tool Face of Steel-A36

D-2 Microphotos of Sample Fabricated with FB Tool Face of Aluminum-Bronze Alloy

D-3 Microphotos of Sample Fabricated with FB Tool Face of Anviloy

APPENDIX E - Friction Bonding Sample Hardness Measurements with Statistics 


\section{$\underline{\text { List of Figures }}$}

Figure 1.1 Monolithic fuel concept......

Figure 1.2 FB tool. The pin extends slightly beyond the shoulder, but not beyond the thickness of the aluminum plate cladding.....

Figure 1.3 Friction Bonding (FB) is used to make fuel plates for the RERTR program........... 6

Figure 1.4 More deformation requires lower temperatures for recrystallization to occur......... 12

Figure 2.1 Schematic drawing of friction stir welding .................................................. 14

Figure 2.2 During FSW: (a) material flow patterns, and (b) processing regions .................... 14

Figure 2.3 Sample cross-sections showing microstructural zones in weld regions................. 16

Figure 2.4 Nugget zone onion rings are apparent in all planes of FSW samples................... 20

Figure 2.5 Cross-sectioned DU10Mo foils prepared at the INL ........................................ 29

Figure 2.6 Bottom face of the FB tool. The tool interior is cooled with ethylene glycol......... 32

Figure 2.7 Two of the raster patterns used for overlapping FB passes during monolithic fuel fabrication at the INL............................................................................. 33

Figure 2.8 Fabrication challenges associated with undesirable FB temperatures. .................. 34

Figure 2.9 Radiographs of FB tungsten particle tracer plates....................................... 35

Figure 2.10 UT scans of fuel plate sets fabricated in staggered arrangement ........................ 36

Figure 2.11 The tool's advancing side is hotter than the retreating side. .............................. 37

Figure 2.12 Debond-UT scan of FB fuel plate set, fabricated using tool face of Steel-A36.... 39

Figure 2.13 X-ray radiograph of FB fuel plate set, fabricated using tool face of Steel-A36.... 39

Figure 2.14 Bend testing is performed on a sample of aluminum-aluminum cladding........... 40

Figure 2.15 Test apparatus used to perform pull tests on fuel plate samples........................ 41

Figure 2.16 Results of mechanical pull tests on monolithic fuel........................................ 41

Figure 4.1 One-sided, single-pass FB process, showing direction of FB tool movement and rotation, with resulting orientation of advancing and retreating edges.................... 47

Figure 4.2 Aluminum plates bonded with one-sided, single pass FB, with varying tool load .. 47

Figure 4.3 Mounted cross-sectioned samples of one-sided, single pass FB aluminum plates,

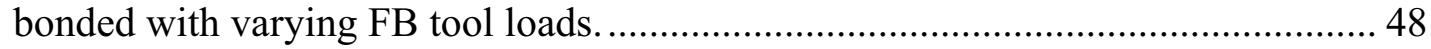

Figure 4.4 Location of Vickers Hardness tests on aluminum samples (to scale) ..................... 1

Figure 4.5 Cutaway view of Side 1 (lower figure) and Side 2 (upper figure) of a surrogate fuel plate, fabricated with a multiple-pass FB process to bond DU10Mo foil inside aluminum Al-6061 cladding.

Figure 4.6 Photos of marked plates, debond-UT scans, and x-ray radiographs for the three plate sets fabricated by friction bonding. 53

Figure 4.7 Mounted cross-sectioned samples of FB DU10Mo monolithic fuel plates, bonded with FB tools faced with different alloys. 
Figure 4.8 Orientation of DU10Mo fuel plate samples mounted in clips.

Figure 4.9 Location of Vickers Hardness tests on cross-sectioned DU10Mo surrogate fuel

sample surfaces (to scale).

Figure 5.1 Hardness measurements on aluminum samples fabricated with one-sided, singlepass $\mathrm{FB}$, with varying FB tool load.

Figure 5.2 Hardness measurements on aluminum samples fabricated with one-sided, singlepass FB, with various FB tool loads.

Figure 5.3 From 50X photos stitched together: Etched samples of two layers (to scale) of Al6061(T6) aluminum joined by one-sided, single-pass FB with varying tool load.... 64

Figure 5.4 Temperature and tool load data illustrative of fluctuations observed during FB of DU10Mo monolithic fuel plate sets.

Figure 5.5 Relative tool work input and coolant heat output during each FB pass

Figure 5.6 Microphotos (50X) stitched together: three cross-sectioned samples (to scale), from surrogate fuel plates fabricated with multiple-pass FB to bond DU10Mo foil inside Al-6061 cladding. 74

Figure 5.7 Hardness measurements on foil surfaces of three cross-sectioned samples............ 76

Figure 5.8 Hardness measurements on aluminum surfaces of three cross-sectioned samples .. 77

\section{$\underline{\text { List of Tables }}$}

Table 2.1 Typical grain characteristics in various zones of a FSW weld region.................... 17

Table 4.1 Samples taken from plates created to represent varied FB fabrication parameters... 44

Table 4.2 Material properties of FB tool face alloys. ......................................................... 51

Table 4.3 Fabrication parameters for surrogate fuel foil and FB plates 52

Table 5.1 Vickers Hardness measurements across aluminum samples fabricated with onesided, single-pass FB.

Table 5.2 Estimates of bonding and grain size, shape, and direction in one-sided, single-pass FB aluminum samples fabricated with varying FB tool loads.

Table 5.3 Average FB tool load and temperature, and tool coolant maximum temperature change, measured during each FB pass on DU10Mo monolithic fuel plate sets fabricated with varying FB tool face alloys.

Table 5.4 Vickers Hardness (HV) measurements across samples from DU10Mo monolithic plates fabricated with differing FB tool face alloys.

Table 5.5 FB tool load and temperature, measured at specific sample locations during FB of DU10Mo monolithic fuel plates fabricated with differing FB tool face alloys.

\section{$\underline{\text { List of Acronyms and Abbreviations }}$}

AFIP ATR Full-size plate In center flux trap Position 
Aluminum

A16061 Aluminum alloyed with magnesium and silicon specific to the aluminum industry's "6061" designation:

\begin{tabular}{|c|c|c|c|c|c|}
\hline Component & Wt. \% & Component & Wt.\% & Component & Wt.\% \\
\hline $\mathrm{Al}$ & $95.8-98.6$ & $\mathrm{Mg}$ & $0.8-1.2$ & $\mathrm{Si}$ & $0.4-0.8$ \\
\hline $\mathrm{Cr}$ & $0.04-0.35$ & $\mathrm{Mn}$ & Max0.15 & $\mathrm{Ti}$ & Max0.15 \\
\hline $\mathrm{Cu}$ & $0.15-0.4$ & Other,each & Max 0.05 & $\mathrm{Zn}$ & $\operatorname{Max} 0.25$ \\
\hline $\mathrm{Fe}$ & Max0.7 & Other,total & $\operatorname{Max} 0.15$ & & \\
\hline
\end{tabular}

Al6061-T4 Aluminum 6061 solution heat treated at above $529^{\circ} \mathrm{C}$, quenched, and then naturally aged at room temperature to allow minimal formation of hardening precipitates, such that the aluminum reaches $1 / 2$ its maximum hardness

A16061-T6 Aluminum 6061 solution heat treated at above $529^{\circ} \mathrm{C}$, quenched, and then annealed at above room temperature to cause formation of hardening precipitates, such that the aluminum reaches $3 / 4$ its maximum hardness

Anviloy A commercially available tungsten alloy

ASM American Society for Materials (Materials Information Society)

ASTM American Society for Testing and Materials

ATR Advanced Test Reactor (located at the INL)

AWS American Welding Society

BAl Boron-Aluminum alloy

BCC Body-Centered Cubic crystalline structure

BR2 Belgian Reactor 2

BU Burn Up, the percent of a nuclear fuel's 235U that is consumed by fission

CEA Commissariat à l'Energie Atomique (in France)

CERCA Compagnie pour l'Etude et la Réalisation de Combustibles Atomiques (in France)

CNEA Comisión Nacional de Energía Atómica (in Argentina)

$\mathrm{Cr} \quad$ Chromium

$\mathrm{Cu} \quad$ Copper

DOE United States Department of Energy

DU Depleted Uranium, i.e. uranium containing less than the natural percentage of ${ }^{235} \mathrm{U}$

DUMo Depleted Uranium - Molybdenum alloy

Efficiency Used in this thesis to indicate relative efficiency of the tool's heat removal from the $\mathrm{FB}$ process, calculated here as: Efficiency $=\mathrm{Q} / \mathrm{W}$

EML Electron Microscopy Laboratory, located at MFC at the INL

FASB Fuels and Applied Science Building

FB Friction Bonding

$\mathrm{Fe} \quad$ Iron 
FSP Friction Stir Processing

FSW Friction Stir Welding

GTRI Global Threat Reduction Initiative

HAZ Heat Affected Zone (in FSW welds)

HB Hardness measured with the Brinell Hardness procedure, or reported on the Brinell Hardness scale

HEU High-Enriched Uranium, $\geq 20 \%{ }^{235} \mathrm{U}$

HFIR High Flux Isotope Reactor (at Oak Ridge, Tennessee)

HIP Hot Isostatic Pressing

HRA Hardness measured with the Rockwell-A Hardness procedure, or reported on the Rockwell-A Hardness scale

$\mathrm{H}_{\mathrm{V}} \quad$ Hardness measured with the Vicker's Hardness procedure, or reported on the Vickers Hardness scale

IAEA International Atomic Energy Agency

INL Idaho National Laboratory

IRIS An irradiation device used to hold experiments in the OSIRIS reactor

LEU Low-Enriched Uranium, $<20 \%{ }^{235} \mathrm{U}$

MFC Materials and Fuels Complex (located at the INL)

$\mathrm{Mg} \quad$ Magnesium

MIT Massachusetts Institute of Technology

Mn Manganese

Mo Molybdenum

MURR Missouri University Research Reactor

$\mathrm{Nb} \quad$ Niobium

NIST National Institute of Standards and Technology

NRC Nuclear Regulatory Commission

OSIRIS A French research reactor named after Osiris, the Egyptian god of the dead

PIE Post-Irradiation Examination

Q Used in this thesis to represent relative heat output from the friction bonding tool coolant, calculated here as: $\mathrm{Q}=\operatorname{Max} \Delta \mathrm{T}$ x Coolant Specific Heat x 1.5 x Pass Time Duration x Coolant Flow Rate

RERTR Reduced Enrichment for Research and Test Reactors

RPM Revolutions Per Minute

SEM Scanning Electron Microscopy

Si Silicon

$\Delta \mathrm{T} \quad$ Maximum change in FB tool coolant temperature between the tool inlet and outlet 
TEM Transmission Electron Microscopy

Ti Titanium

TLPB Transient Liquid Phase Bonding

TMAZ Thermo-Mechanical Affected Zone (in FSW welds)

TTI Transformation Technologies Incorporated, a company specializing in FSW equipment

TUM Technical University of Munich, Germany

U Uranium

${ }^{235} \mathrm{U} \quad$ Uranium-235, an isotope with only 235 protons in its nucleus rather than a stable amount of 238 protons

U10Mo Uranium alloyed with 10wt\% Molybdenum

U7Mo Uranium alloyed with 7wt\% Molybdenum

UMo Uranium - Molybdenum alloy

UT Ultrasonic Testing

W Used in this thesis to represent relative work input by friction bonding, calculated here as: $\mathrm{W}=$ Average Load $\mathrm{x}$ Traverse Speed $\mathrm{x}$ Pass Time Duration

Zn Zinc

$\mathrm{Zr} \quad$ Zirconium 
Microstructural Examination to Aid in Understanding

Friction Bonding Fabrication Technique

for Monolithic Nuclear Fuel 



\section{Microstructural Examination to Aid in Understanding Friction Bonding Fabrication Technique for Monolithic Nuclear Fuel}

\section{INTRODUCTION}

New nuclear fuel designs and fabrication techniques are under development by the Reduced Enrichment for Research and Test Reactors (RERTR) program at the Idaho National Laboratory (INL). "Monolithic" fuel is a new design under development, and one alternate bonding fabrication technique being investigated to make monolithic fuel is friction bonding (FB). This is a new variation of friction stir welding (FSW), a metal bonding technique practiced since about 1991, but never before used in nuclear fuel fabrication. Monolithic fuel plates have been tested in only a few reactors to date, and FB has not been performed anywhere other than the INL.

To help better understand this new FB process and its potential application to monolithic fuel production, surrogate fuel plates were fabricated with varying FB fabrication parameters. The topic of this thesis study is the microstructural examination of two sets of unirradiated surrogate FB monolithic fuel plate samples. The first sample set was taken from plates fabricated using various FB tool loads to prepare one-sided, single-pass FB aluminum cladclad plates. The second sample set was taken from fuel plates fabricated by using FB tool faces made of different metal alloys. Samples were destructively taken from these plates and examined, with a focus on microstructural differences between samples in each set. This was new and original research, not performed anywhere prior to this study.

These examination results are now available to researchers for comparison with existing data on similar monolithic fuel samples made with other fabrication techniques, or with the same fabrication techniques on different manufacturing equipment. Results can also be compared with post-irradiation examination data on the real fuel. Results will help define future areas of research, and can help determine whether and how FB can be used to fabricate nuclear fuel plates. If monolithic fuel fabricated by FB is recommended for use in reactors, these examination results will be part of the qualification package submitted to the Nuclear Regulatory Commission (NRC) for validation of the fuel design.

To aid understanding of this study topic, this Introduction section provides general background information on the RERTR program, monolithic fuel design and fabrication techniques, fuel examination methods, and material microstructure and properties. Section 2 includes more detailed information on the current state of the art of FSW and FB fabrication and RERTR fuel development efforts. Subsequent sections provide the thesis objective, the experimental materials and methods, results, conclusions, and recommendations. References and appendices are included at the end of the report.

\subsection{Reduced Enrichment for Research and Test Reactors (RERTR) Program Mission}

Research reactors around the world have very different fuel designs from those of power reactors. One significant difference is that research reactor fuel is more highly enriched in the isotope uranium-235 $\left({ }^{235} \mathrm{U}\right)$, and thus provides higher power density over a much smaller volume in comparison to power reactors. "Enriched" uranium has been processed such that the weight-percent composition of the isotope ${ }^{235} \mathrm{U}$ is higher than is found in natural uranium, which typically has only $0.72 \%{ }^{235} \mathrm{U}$. Research reactor fuel enrichment is often above $20 \%$ ${ }^{235} \mathrm{U}$, and can range to greater than $90 \%{ }^{235} \mathrm{U}$, much higher than that found in power reactors fuels, which typically have $3-6 \%{ }^{235} \mathrm{U}$ enrichment. 
Reactor fuels are fabricated in only a few countries ${ }^{1}$, and thus must be shipped across the world. The quantity of research reactor fuel produced and shipped worldwide is much less than that of power reactor fuel. However, because their ${ }^{235} \mathrm{U}$ enrichment is much higher, the shipment and use of research reactor fuels pose greater associated nuclear weapons proliferation risks; bombs can be made from highly enriched uranium.

Uranium can be separated relatively easily from other materials in the fuel plates by chemical separation processes. If the uranium is already highly enriched in ${ }^{235} \mathrm{U}$, it could then be used directly to make a nuclear bomb. However, if the uranium has a low percent of ${ }^{235} \mathrm{U}$, it must be further processed to enrich its ${ }^{235} \mathrm{U}$ content before it could be used to make a bomb. The enrichment process is a difficult procedure requiring expertise, capital equipment, and time, and poses a deterrent for nuclear weapons development. Therefore fuels with lower ${ }^{235} \mathrm{U}$ enrichment pose less risk for proliferation of nuclear weapons.

In response to a call and strategy by the International Atomic Energy Agency (IAEA) to reduce worldwide proliferation risk, the international and national nuclear communities and their agencies have set a goal of substantially reducing enrichment levels in research reactor fuels. In order to reduce international traffic of high-enriched uranium (HEU, $\geq 20 \%{ }^{235} \mathrm{U}$ ) used to fuel research reactors around the world, the IAEA and the United States are encouraging and applying political pressure for all nations to change their research reactor operations and licensing to use fuel of low-enriched uranium (LEU, $<20 \%{ }^{235} \mathrm{U}$ (Clark and Briggs 2004)). As reactor-owning nations around the world are stepping up to the challenges, the fuel-producing nations have likewise committed to changing the fuel design. The United States has been pushing this ambitious proposal for reduced fuel enrichment, and is now under international and national political pressure to show good faith and meet its commitments in turn.

As part of the U.S Department of Energy's (DOE's) Global Threat Reduction Initiative (GTRI), the RERTR program at the INL was started in 1978 to support the commitment to develop LEU fuel designs for research reactors, and develop the technology needed to allow conversion of these reactors to the new fuel. (Clark and Briggs 2004) The most challenging aspect is developing LEU fuels that can provide the same power density as the most highly enriched fuels currently used in research reactors. To accomplish this, the same amount and volume density of ${ }^{235} \mathrm{U}$ is required in the reactor. Therefore the new fuel must contain the same amount of ${ }^{235} \mathrm{U}$, but the ${ }^{235} \mathrm{U}$ must be integrally mixed with much more ${ }^{238} \mathrm{U}$ to comprise an overall lower percent. So, the new fuel as a whole must contain much more uranium, and therefore is heavier. However, any reactor fuel conversions must be done without significant penalty in reactor performance or safety and involve, at most, minimal geometry changes in the fuel element or core design. (Clark, Meyer and Hayes 2002)

Initial RERTR program development efforts and introduction of LEU fuel types allowed about 40 reactors to be converted to or originally built using LEU fuel. However, the RERTR fuel development work halted in 1989, and there remained many reactors that were still not able to convert to LEU fuel because their ${ }^{235} \mathrm{U}$-density requirements were not met by the LEU fuels developed to that point. Thus the RERTR advanced fuel development program began in 1995. (Clark, Meyer and Hayes 2002) The goal of the RERTR advanced fuel development program is to complete the conversion of all targeted research reactors by 2014. (DeMint, et al. 2007)

\footnotetext{
${ }^{1}$ Research reactor nuclear fuel is supplied by Argentina, France, Russia, and the United States.
} 
One higher-density fuel design introduced by the RERTR advanced fuel program is the dispersion fuel concept. Dispersion fuel consists of the fuel in a powder form dispersed in an aluminum matrix that is clad between two aluminum cover plates. (Clark and Briggs 2004) The dispersion fuel design appears very promising for providing the ${ }^{235} \mathrm{U}$ density required for successful conversion of all remaining HEU research reactors to LEU fuel, except for a few (28 worldwide, including five in the U.S. ${ }^{2}$ ) that need even higher ${ }^{235} \mathrm{U}$-density. Collaboration with industry is underway to ensure the dispersion fuel technology is commercially available from multiple fuel vendors at completion of the qualification effort. (D. M. Wachs, D. D. Keiser, et al. Sept 2007)

The very highest ${ }^{235} \mathrm{U}$-density LEU fuel can be provided by a design with fuel meat in the form of uranium foil encased in aluminum (Al) cladding, as illustrated in Figure 0.1. This foil form is described as "monolithic" fuel, distinguished from conventional fuel designs where the uranium is typically dispersed in a non-uranium matrix to form the fuel meat. The solid monolithic foil clearly offers a higher density of uranium than dispersion fuels can. However, knowledge gained during dispersion fuel development has been usefully applied to the monolithic fuel development effort. When development is successfully complete, monolithic fuels will be available if desired to provide the ${ }^{235} \mathrm{U}$-density needed for conversion of all research reactors, including the remaining few research reactors using very high HEU.

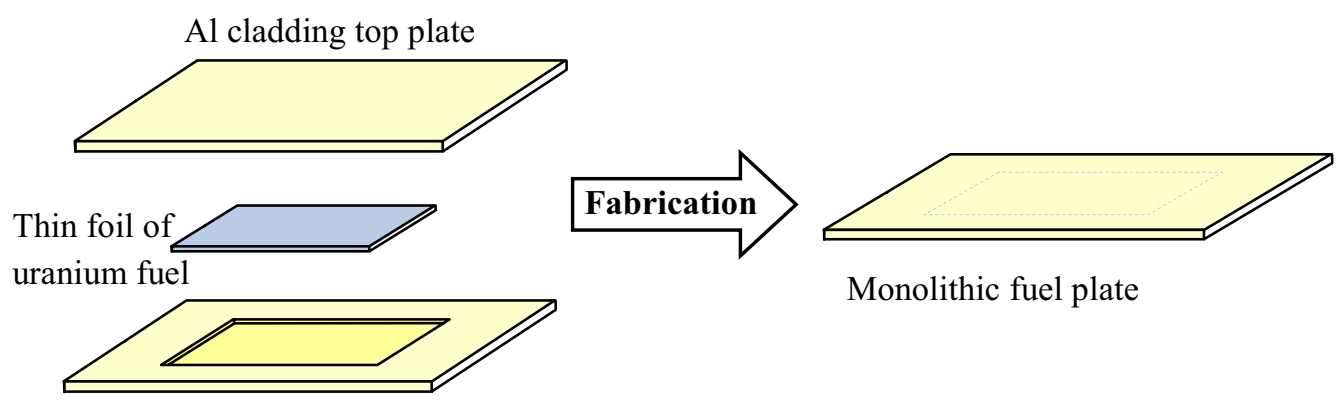

Al cladding bottom plate with milled pocket

Assembly

Figure 0.1 Monolithic fuel concept.

\subsection{Monolithic Fuel Design}

Monolithic fuel has several advantages. As mentioned, the foil form provides a high density uranium fuel, yielding enough $\mathrm{U}^{235}$ per volume to meet reactor power requirements even when the fuel is LEU. The single-piece fuel foil also provides a considerably lower interface area between fuel and aluminum than occurs in dispersion fuel, which has both aluminum alloy (A1-6061) cladding and aluminum matrix material. The lower interface area allows less uranium-aluminum chemical reaction. Formation of a chemical reaction layer at the

\footnotetext{
${ }^{2}$ United States reactors requiring fuel with higher ${ }^{235} \mathrm{U}$ density than dispersion LEU fuel can provide are: the Missouri University Research Reactor (MURR), the Massachusetts Institute of Technology (MIT) reactor, the National Institute of Standards and Technology (NIST) reactor near Washington DC, the High Flux Isotope Reactor (HFIR) at Oak Ridge National Lab in Tennessee, and the Advanced Test Reactor (ATR) at the INL.
} 
interface is detrimental, in part because it creates a thermal barrier with resulting rise in fuel temperature. This elevated fuel temperature decreases fuel performance and promotes even more reaction layer formation, exacerbating the problem. In addition, gaseous products from the fission of ${ }^{235} \mathrm{U}$ tend to collect in the reaction layer material; this weakens the interface and can lead to fuel-aluminum bond delamination or breakaway swelling [(Clark and Briggs 2004) and (Burkes, Keiser and Miller, et al. January 2007a)]. More information about the interface reaction layer can be found in Appendix A.

The highest priorities in monolithic fuel design and fabrication are to provide: (1) correct

${ }^{235} \mathrm{U}$ density to maintain the same reactor power with LEU enrichment levels; (2) good bond strength between the aluminum cladding layers so the fuel meat is well-contained within the aluminum and cannot migrate elsewhere in the reactor; (3) good bond strength between the aluminum cladding and uranium fuel meat itself so that thermal conductivity is maintained across the bond; this includes minimal reaction layer formation at the interface between the fuel and aluminum, as such an interface layer degrades thermal conductivity and bond strength at that location; (4) homogeneous material phase and grain properties throughout the fuel to assure uniform production of gaseous fission products and minimal fuel swelling; and (5) easy implementation of a fabrication method with least complexity and capital outlay.

\subsection{Monolithic Fuel Fabrication}

The composition and dimensions of monolithic fuel are designed to meet the first priority. Whether the remaining priorities are met depends largely on the monolithic fuel bonding fabrication technique. One of the biggest challenges associated with monolithic fuel is development of a suitable technique to encase and bond the foil inside cladding metal without introducing undesirable changes in material properties. The only fuel cladding material used in United States research reactors is aluminum alloy Al-6061, which provides sufficient strength, good thermal conductivity, and good transparency to neutrons. Significant differences between the aluminum cladding and the uranium foil ductility and thermal properties make conventional roll-bonding techniques inadequate for bonding the foil inside the cladding; roll bonding does not provide correct relative thicknesses of foil and aluminum, and causes unacceptable tearing and intermittent bonding of the foil within the cladding.

Three alternate fabrication techniques for monolithic fuel have been investigated by the INL RERTR Program and are being evaluated relative to the above priorities. Variations within each of these techniques have been explored with different temperatures, pressures, and additives [(Clark, Jue, et al. October 2006), (Keiser, Jue and Clark 2006), and (Clark, Moore, et al. 2007)].

- TLPB (Transient Liquid Phase Bonding) - This metal bonding technique uses a thin foil interlayer between surfaces of components to be joined. The assembly is heated to melt the foil, which diffuses into the component substrates and induces melting; the liquid solidifies as mixing and homogenization occurs and the mixture's liquidus temperature rises.

Because a TLPB fuel plate cladding lost its bond integrity and released fuel material into the test reactor's coolant during irradiation in 2006, that form of the TLPB fabrication technique is no longer under consideration. Modified applications are being considered. (Clark and Briggs 2004)

- HIP (Hot Isostatic Pressing) - This process subjects the fuel plate assembly to both high heat and evenly applied high pressure in an argon gas containment vessel. The simultaneous application of high temperature and pressure eliminates internal voids and creates bonds through plastic deformation, creep, and diffusion bonding. The HIP 
fabrication technique has been used since 1964 in nuclear fuel manufacture. It shows good promise in producing monolithic fuel plates with consistent dimensions and material properties, and a strong development program is underway. However, the high heat necessary for the HIP process promotes formation of a U-Al reaction layer. If HIP is performed at lower temperatures to avoid this phenomenon, it must be run for longer times; the high temperatures, interface layer formation, and long fabrication times are relative disadvantages of the HIP process, as is the capital cost for the huge HIP equipment that would be necessary for fabrication of full sized fuel plates. (Clark and Briggs 2004)

- FB (Friction Bonding) - This metal bonding technique, a new variation of friction stir welding (FSW), utilizes a circular tool with a shoulder and a small center pin as illustrated in Figure 1.2. When the tool is rotated and applied to the surface of components to be joined, its center pin penetrates into the metal, while its rotating shoulder rubs on the top metal surface, and presses down with a forging action. The resulting mechanical deformation and frictional heat create temperatures that are less than the metals' melting temperatures, but still sufficient to create physical (not chemical) bonds between the metal components. The key to friction welding in general is that no molten material is generated, the weld being formed in the solid state. The FSW process was developed by TWI, Ltd. at Cambridge University in England in 1991(Thomas, et al. 1991), but has never before been used to make nuclear fuel.

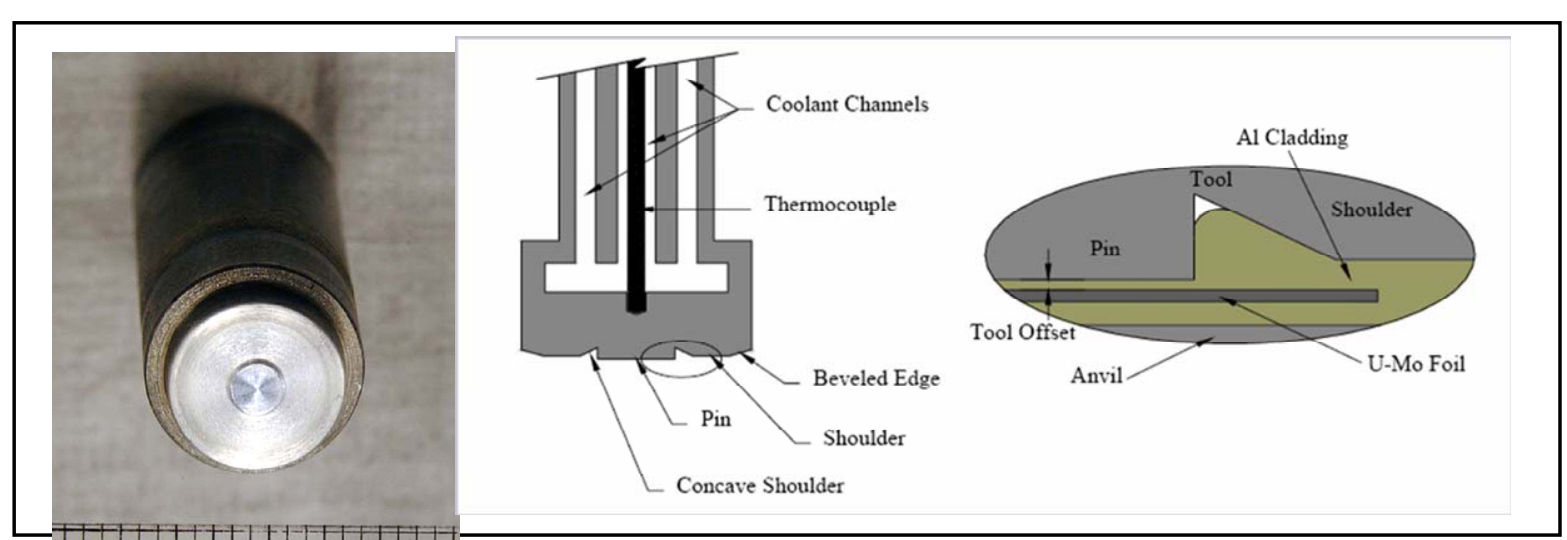

Figure 0.2 FB tool. The pin extends slightly beyond the shoulder, but not beyond the thickness of the aluminum plate cladding.

[(Clark, Hallinan, et al.9May 2006) and (Dixon, Burkes and Medvedev 2007)]

Uranium (U) mixed with molybdenum (Mo) is a promising fuel alloy for research reactors, since addition of 7-12 $\mathrm{wt} \%$ Mo stabilizes the fuel microstructure and reduces U-Al reaction layer formation and swelling (Clark, Jue, et al. October 2006). In monolithic fuel fabrication, the UMo alloy is cast and rolled into foil, annealed, and clad in Al-6061 via one of the above fabrication techniques. After that the plate is inspected, subjected to irradiation, and then examined. Monolithic fuel was first fabricated by roll-bonding, and irradiated as part of the RERTR-4 test series in 2001. The RERTR-6 experiment in 2005 was the first irradiation of monolithic fuel (made by FB) where the foil occupied the full fuel meat volume in the miniplate (Finlay, Wachs and Hofman 2006). FB monolithic fuel plates have been included in each subsequent RERTR test series, including the current RERTR-9 fuel plates. With each test series, surrogate fuel plates are typically fabricated with the same fabrication process as the actual fuel plates, but with depleted uranium (DU) in place of enriched-uranium fuel. These surrogates are not irradiated, but are archived for future reference and analysis. This thesis study included examination of samples taken from surrogate plates representing the RERTR9A experiment. 


\subsection{Friction Bonding of Monolithic Fuel}

The RERTR Program's friction bonding (FB) technique is illustrated in Figure 1.3. Photos of the associated FB equipment and resulting bonded plates can be found in Appendix B Figures B-2 and B-4, respectively. At the INL, FB fuel is fabricated at the Fuels and Applied Science Building (FASB) at the Materials and Fuels Complex (MFC). First two pieces of aluminum cladding plate are stacked with a fuel foil nested in a pocket between them. Next, the FB tool is rotated and its center pin is plunged into the surface of the top aluminum plate until the tool shoulder comes to rest on the plate's top surface. The pin does not extend through the top plate, but pressure and frictional heat between the rotating tool and the aluminum cause the latter to soften without reaching the melting point. This softening allows traversing of the tool through the top aluminum plate, during which a solid phase bond is formed with the layer of metal below it forged by the downward pressure exerted from the tool. Multiple overlapping traversing passes are made until the entire plate surface has been subjected to the FB process. Then the assembly is turned over and the FB process is repeated on the opposite side; this assures the foil and cladding are well bonded on both sides, and also that material and grain properties are symmetrical across the fuel plate cross-section. Compared with HIP, potential advantages of the FB technique for monolithic nuclear fuel fabrication include lower initial capital cost for fabrication equipment, relatively faster production time, fewer hightemperature effects on material properties, and minimal formation of a U-Al reaction layer.

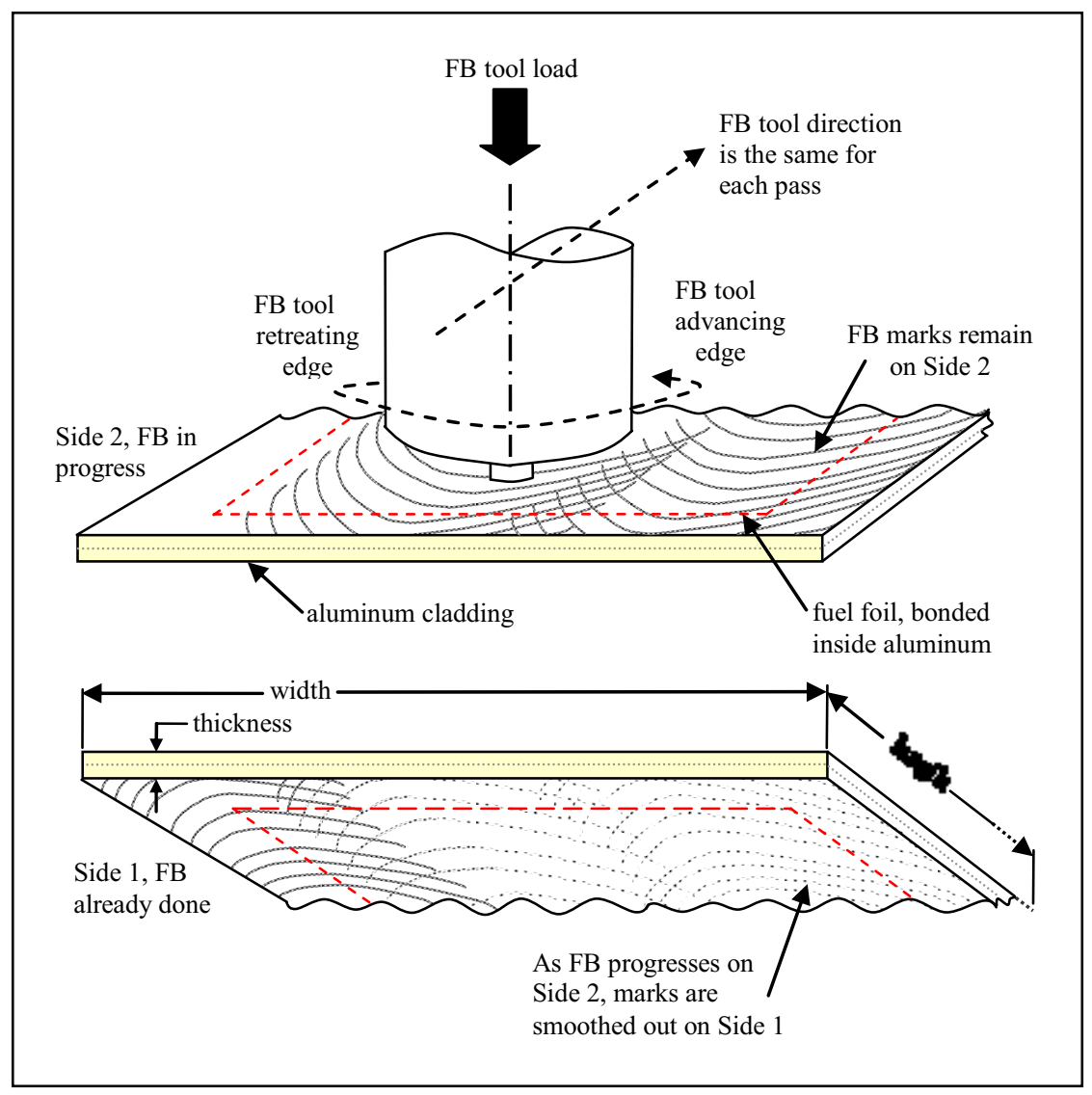

Figure 0.3 Friction Bonding (FB) is used to make fuel plates for the RERTR program by performing multiple-pass FB, first on Side 1 (lower figure) and then Side 2 (upper figure) of a fuel plate, to bond fuel foil inside aluminum Al-6061 cladding. 
Fuel plate external dimensions are prescribed by reactor designs, while internal dimensions and composition are driven by the reactor power level requirements and enrichment goals. However, some FB operational parameters may be varied during fuel fabrication. These parameters include the load applied to the tool face, the tool face material, and rotational and traversing speeds. Prior to this thesis study, the effects on fuel properties of varying the FB fabrication parameters were unknown, particularly with respect to bonding and grain structure. Thus optimal parameter values or ranges of values were also unknown.

\subsection{Fuel Examination Methods}

Several examination methods can be used to characterize and evaluate fuel plates, although not all methods have been used on all plates. Since bond quality - for both fuel-cladding and cladding-cladding bonds - is crucial to successful performance of a fuel plate, it is an important part of fuel plate evaluations. The RERTR program's fuel examination methods can be classified as either irradiation-performance evaluation or pre-irradiation examination methods:

Irradiation-Performance Evaluation Methods

- Modeling of irradiation performance uses finite element analysis and analytical solutions relating to both thermal and thermo-mechanical aspects of the fuel-cladding interface. This method can investigate a debond's impact on fuel meat temperature and stress behavior, thus supporting determination of acceptable debond size and geometry [(Wachs, Burkes, et al. Oct 2006b) and (Burkes, Keiser and Miller, et al. January 2007a)].

- Post-irradiation examination (PIE) involves both non-destructive measurement of plate thickness and destructive examination of microstructure via metallography ${ }^{3}$ of cross-sectioned fuel plates. The PIE reveals interface reaction layer development, changes in grain size, material swelling, fission gas bubble formation, and bond delamination (Finlay, Wachs and Hofman 2006).

- Pre-irradiation Examination Methods

- Ultrasonic Testing (UT) is routinely performed on every fuel plate. It is non-destructive and provides information on bond quality in an efficient manner. It can determine whether debonds are present (via 'debond-UT') and the location of the foil inside the fuel plate (via 'minclad-UT') (Clark, Jue, et al. October 2006).

- $\quad$ Radiography inspection of fuel plates is another non-destructive method. Every fuel plate is x-rayed to identify the boundaries of the fueled region, to detect fuel outside those boundaries, and to measure fuel density with respect to standard specimens. It does not indicate debonds.

- Mechanical tensile or shear testing:

- Bend testing of cladding-cladding bonds is performed by clamping a thin section of the non-fueled region of the plate into a fixture and incrementally bending it one direction over a small radius for $90^{\circ}$, back for $180^{\circ}$, then returning it to its original orientation. If any visual delamination is noted, the bond is considered unacceptable. Cladding-cladding bonds created by all three bonding fabrication methods have successfully passed the bend testing (Clark, Jue, et al. October 2006).

\footnotetext{
${ }^{3}$ Microstructural examination of metal samples is often termed "metallography."
} 
- Instrumented tensile testing of a bond interface is performed by pull testing a fuel plate specimen so that its two cladding surfaces, on either side of a surrogate fuel foil sandwiched between, are pulled directly apart from each other (i.e. in the normal rather than shear direction). This is similar to the American Society for Testing and Materials test method given in (ASTM 2006a). [(Burkes, Keiser and Miller, et al. January 2007a) and (Burkes, Keiser and Wachs, et al. March 2007)].

- Microstructural examination is performed via metallography of cross-sectioned surrogate fuel plates, focusing on the cladding-cladding and fuel-cladding interfaces. Many of the same parameters are inspected as during the PIE microstructure examination: interface reaction layer development, material swelling, grain size, hardness testing, and bond delamination (Keiser, Jue and Burkes 2007).

Over the years, the above examinations have been conducted on various combinations of actual and surrogate fuel plates. Some records are available on fabrication techniques and parameters but there are gaps in the data, and therefore incomplete understanding of the impact that some changes in fabrication parameters have on fuel plate characteristics. For example, it is not clear whether some bond phenomena detected during PIE occurred as a result of irradiation or from the fabrication process. As fuel development work evolved, it became appropriate to adopt a more rigorous approach to compile archive and fabrication data. Starting in 2006 such data were gathered on the RERTR-8 and RERTR-9 test series; the microstructural examination performed by this thesis study is part of that data gathering and analysis. During the ensuing time, RERTR-8 fuel plates have been irradiated and PIE is underway, while RERTR-9 fuel plates are currently undergoing irradiation.

\subsection{Material Microstructure and Properties}

(Flinn and Trojan 1975)

To understand the goals and results of microstructural examination, it is helpful to understand some fundamentals of material properties. Many of these properties are driven by the material microstructure, even from an atomic level.

\subsubsection{Crystalline Phases}

A solid material crystal is composed of atoms arranged in an orderly recurring geometric lattice, repeated continuously throughout the crystal. Common metals typically have crystals with relatively simple lattice structures, while uranium has crystals with more complicated lattice structures: orthorhombic (termed " $\alpha$-uranium"), tetragonal (" $\beta$-uranium”), or bodycentered cubic ("BCC" or " $\gamma$-uranium"). A material solid state with homogeneous lattice structure throughout is called a solid phase, e.g. $\gamma$-phase, $\beta$-phase, and $\alpha$-phase for uranium. Different phases exist under different conditions of pressure and temperature. When the conditions change, the phase can change, with resulting material volume changes.

Pure uranium at room temperature exists in $\alpha$-phase, a crystal lattice with different properties in the $\mathrm{X}, \mathrm{Y}$, and $\mathrm{Z}$ axes because of different recurring patterns of the atoms on each axis. When heated the uranium expands in the $\mathrm{X}$ and $\mathrm{Z}$ directions and shrinks in the $\mathrm{Y}$ direction; heating and cooling of $\alpha$-phase uranium can lead to drastic dimensional changes and gross distortions of the metal. Thus, pure uranium in $\alpha$-phase is not used as a fuel, but only in alloys or compounds. (DOE 1993) Uranium used in monolithic fuel is in $\gamma$-phase and is alloyed with molybdenum; more information about this is available in Appendix A. 
NOTE: A revision to this report section (1.6.1 Crystalline Phases) was offered by Dr. Thomas Hartmann ${ }^{4}$ to provide clarification in the crystallographic sense:

Crystalline solid matter is characterized by its far-ordered atomic arrangement in the crystal structure. Atoms occupy lattice positions of the crystal lattice which is defined by symmetry operations in regard to the realized space group. As a result the highly symmetric atomic arrangements in crystalline solid materials are far-ordered and the structural motifs are repeated continuously through the crystals. In most metals rather high-symmetric structural arrangements are realized, and space groups of highest symmetry content in the cubic and hexagonal crystal systems are preferred. Uranium however is polymorph and as a function of temperature crystallizes in three different allotropic forms: (1) $\alpha-\mathrm{U}$ in the temperature range $20^{\circ} \mathrm{C}$ to $668^{\circ} \mathrm{C}$ is orthorhombic, (2) $\beta$-U in the temperature range $668^{\circ} \mathrm{C}$ to $775^{\circ} \mathrm{C}$ is tetragonal, and $(3) \gamma-\mathrm{U}$ in the temperature range $775^{\circ} \mathrm{C}$ to $1135^{\circ} \mathrm{C}$ is cubic with a body-centered-cubic (bcc) structure type. Phase transformation between the allotropic forms of uranium is associated with minor changes in volume and density. Hereby $\alpha-U$ has a density of $19.04 \mathrm{~g} / \mathrm{cm}^{3}$ (at 25 ${ }^{\circ} \mathrm{C}$ ) while $\beta-U$ and $\gamma-U$ have densities of $18.11 \mathrm{~g} / \mathrm{cm}^{3}$ (at $720{ }^{\circ} \mathrm{C}$ ) and $18.06 \mathrm{~g} / \mathrm{cm}^{3}$ (at $805^{\circ} \mathrm{C}$ ), respectively.

Pure uranium at room temperature exists as orthorhombic $\alpha$-uranium with lattice parameters $a=2.854 \AA, b=5.869 \AA$, and $c=4.955 \AA$, and physical properties that will depend on crystallographic orientation of the material. When heating $\alpha$-uranium, lattice parameters $a$ and $b$ will expand while lattice parameter $c$ contracts. In operations where $\alpha$-uranium undergoes alternating heating and cooling cycles, stress fractioning and gross distortion of the metal are observed. Thus, pure uranium in the $\alpha$-modification is not used as a fuel, but only in alloys or compounds.

\subsubsection{Strain and Strength}

Material subjected to a stress will deform; the amount of deformation relative to its original size is called "strain." If the material returns to its original dimensions when the load is removed, the deformation is "elastic" strain. However, any amount of permanent deformation is "plastic" strain. A material's strength at any certain temperature is defined by the amount of stress it can withstand without occurrence of plastic strain.

A row of missing atoms in a crystal lattice is termed a "dislocation." Atoms near a dislocation are not firmly held in place, and can more easily move along the dislocation, creating a "slip plane." When materials are cold-worked, e.g. reshaped by rolling, millions of dislocations are formed. As more slip occurs, the dislocations interact, pile up and dislocation tangles form. This makes it more and more difficult for further slip to take place. When metal is cold-worked, it is reshaped by plastic strain, and the material does not return to its original dimensions. The cold-working process uses up all the slip planes and dislocation sites where easy slip could occur, and when the load is removed, there is no change in this situation. So when a load is later reapplied, no plastic strain is possible until a stress level is reached that is higher than the stress previously applied during the cold-work; thus materials with more dislocations are stronger.

\footnotetext{
${ }^{4}$ Per personal communication with Dr. Thomas Hartmann on April 18, 2008.
} 
A material grain is the space occupied by a continuous crystal lattice, even if the lattice contains numerous dislocations. A grain is merely a crystal without smooth faces because its growth was impeded by contact with another grain or a restraining surface. Grain boundaries are areas of high dislocation density and entanglements and are impediments to slip. When cold-working occurs and dislocations form, new grain boundaries can also form, resulting in finer (smaller) grains.

\subsubsection{Hardness}

Hardness measurements depend on resistance to plastic deformation. The hardness of a material is higher after cold working because dislocation density is high and the sites for easy slip have been used up. Thus there is good correlation between hardness and strength.

In addition to dislocation density, another microstructural characteristic that can make alloyed materials (such as Al 6061) much stronger and harder is the presence of finely dispersed alloy precipitates within the grains. Precipitation hardening occurs when atom spacing in the precipitate is close to a spacing in the matrix. A region of strained material then surrounds the precipitate particle, making it difficult for slip to occur in this region, and resulting in increased strength and hardness.

Hardness is not an intrinsic material property defined in terms of fundamental units. Rather, a hardness value is the result of a measurement taken per a defined procedure, which usually measures the depth or area of an indentation left by an indenter of a specific shape, with a specific force applied for a specific time. The three principal standard test methods are Vickers, Brinell, and Rockwell. (GordonEngland 2008)

The Vickers hardness test method indents the test material with a diamond indenter, in the form of a right pyramid with a square base and an angle of $136^{\circ}$ between opposite faces, subjected to a load, normally applied for 10 to 15 seconds. The area of the sloping surface of the indentation is calculated based on the diagonals of the indentation. The Vickers hardness $\left(\mathrm{H}_{\mathrm{V}}\right)$ is the quotient of the load $(\mathrm{kgf})$ divided by the indentation area $\left(\mathrm{mm}^{2}\right)$. (GordonEngland 2008)

Vickers microhardness measurements were taken on material samples as part of this thesis study. The term "microhardness" usually refers to indentations made with loads not exceeding $1 \mathrm{kgf}(2.2 \mathrm{lbf})$. The procedure for Vickers microhardness testing uses the same diamond pyramid, and is very similar to the standard Vickers hardness test except that it is done on a microscopic scale with higher precision instruments. The surface being tested generally requires a metallographic finish; the smaller the load used, the higher the surface finish required. Precision microscopes are used to measure the indentations; these usually have a magnification of around $500 \mathrm{X}$ and measure to an accuracy of $\pm 0.5 \mu \mathrm{m}$. (GordonEngland 2008)

Advantages of the Vickers hardness test method are that extremely accurate readings can be taken, and just one type of indenter is used for all types of metals and surface treatments. The Vickers method is very adaptable and precise for testing the softest and hardest materials under varying loads. (GordonEngland 2008) 


\subsubsection{Temperature Effects}

The term "cold" in cold-working is relative. It means working at a temperature that does not alter the strain, strength and hardness characteristics produced by the work. In other words, cold work causes atoms to move and dislocation tangles to form, and this effect can be removed by working at higher temperatures.

We do not always want maximum strength and hardness, because as the hardness increases the ductility decreases. If the metal is heated, e.g., annealed in a furnace, the added energy allows atoms in severely strained regions to regroup and move to unstrained positions, dislocations and slip planes can disappear, and the metal can thus be restored to its original strength and ductility. This is adjustment to strain on a microscopic scale, and the overall formed shape does not change dimensions.

Temperature effects on cold-worked material can be considered in three stages, in order of increasing temperature:

- In the "recovery" temperature range, stresses are relieved in the most severely slipped regions and dislocations move to lower energy positions. Hardness and strength may not change greatly, but corrosion resistance is improved.

- In the "recrystallization" range, grains deformed by plastic strain are replaced by a new set of undeformed grains that nucleate and grow until the deformed grains have been entirely consumed. The new grains have low dislocation density, and so recrystallization is usually accompanied by a reduction in the strength and hardness of a material and a simultaneous increase in the ductility. Recrystallization is driven by the material's temperature; at moderate to high temperatures recrystallization occurs readily, particularly in materials such as aluminum and nickel. "Dynamic" recrystallization occurs when the nucleation and growth of new grains takes place during the deformation process rather than afterwards as part of a separate heat treatment. Recognizing the effects of dynamic recrystallization is helpful to understanding microstructure observed in FSW or FB samples.

- In the "grain growth" range, grains continue to enlarge as the temperature is raised further. Material with larger grains has less number of grain boundaries and therefore lower dislocation density. So hardness and strength are typically decreased and ductility increased. Thus, if a cold-worked part is heat treated, the metal softens as a function of temperature and time because the grain size becomes larger.

In short, cold-worked material with small grains is typically stronger, harder, and less ductile than material with larger grains due to the higher number of grain boundaries with an associated high density of dislocations. However, when the material undergoes heat treatment, recrystallization causes the old grains to be replaced by new stress-free crystals which provide lower strength and hardness than the original material. There is an interesting relation between the amount of previous cold work and the grain size of the recrystallized material; a greater amount of cold-work yields more nuclei for the new grains, and the resulting grain size is smaller. Related to this, the greater the amount of deformation due to previous cold work, the more energy is stored in the deformed grains, therefore, the lower the temperature needed for recrystallization to occur, as shown in Figure 0.4. 


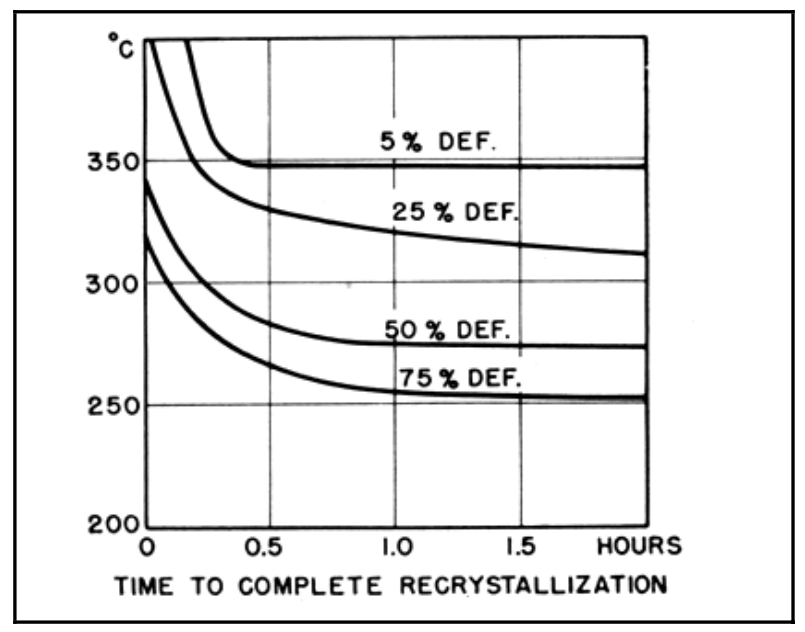

Figure 0.4 More deformation requires lower temperatures for recrystallization to occur [(Schneider 2002), from (Hultren 1952)].

In addition to temperature effects on dislocations, slip planes, and grain size, temperature also impacts the hardening precipitates. When a hard material is heated to an elevated temperature, the hardening precipitates easily dissolve into the matrix; if the material is then quenched, its low-precipitate condition is "frozen," and the final material is thus softer than the original. While precipitates want to dissolve at high temperatures, they conversely want to form and exist at lower temperatures. Therefore, dissolved precipitates in a quenched material will often reform slowly at room temperature (termed "natural aging"), or more quickly for a time spent at higher-than-room temperature (termed "tempering"), and hardness and strength will be at least partially recovered. 


\subsection{LITERATURE SEARCH: STATE OF THE ART IN FRICTION BONDING AND ITS APPLICATION TO FUEL PLATE FABRICATION}

Friction Stir Welding (FSW) has been performed since 1991 (Thomas, et al. 1991), and innumerable studies have been reported investigating many aspects and process variants. One FSW process variant is FB, currently under development at the INL and not yet performed anywhere else, so literature discussing FB has been generated primarily at the INL. This section discusses the state of the art of FSW aspects which seem most pertinent to understanding the FB process being developed at the INL, and especially helpful in understanding examination results observed in this study. It also discusses the state of the art of monolithic fuel development and FB.

\subsection{Friction Stir Welding (FSW)}

FSW is a relatively new solid-state welding process, particularly suited to aluminum alloys. Aspects which seem most pertinent to understanding the FB process being developed at the INL, and especially helpful in understanding examination results observed in this study, include what is known to happen to aluminum during FSW, i.e. it plasticizes and is extruded and mixed across the bond line. FSW changes the material microstructure grain size, direction, precipitate distribution, hardness, and strength. Different combinations of process parameters cause various thermal conditions, evidenced by differences in the microstructure and efficacy of bonding.

\subsubsection{FSW Process and its Development}

An excellent state of the art review of FSW technology is provided by (Mishra and Ma 2005), and the process is illustrated in Figure 2.1. FSW utilizes a non-consumable rotating shouldered pin tool, of harder material than the workpieces being welded, and whose pin length is slightly less than the weld depth required. The rotating pin makes contact and is plunged into the joint line between two metal plates - typically either butted together or overlapping - until the tool shoulder is in intimate contact with the work surface. Heat is generated due to both friction and extrusion that occurs between the rotating welding tool and the plate material. This heat causes the latter to plasticize (soften without reaching the melting point) in the immediate region of the work pieces, and allows traversing of the tool along the weld line. As the rotating tool is moved forward in the direction of welding, plasticized material flows around the tool, coalesces, and is forged behind the tool where it cools and forms the solid-state weld. Figure 2.2 illustrates material flow patterns and the processing regions that occur during FSW. [(Gould and Feng 1998), (Marzoli, et al. 2005), (Mishra and Ma 2005), (W. J. Arbegast 2007), (Chen 2007), (Querin, Davis and Schneider 2007), and (TWI 2007)]

During FSW, one edge of the tool shoulder both rotates and traverses in the same direction; this edge is termed the "advancing" edge. The other edge rotates in a direction opposite to, i.e., retreating from, the traversing direction; this edge is called the "retreating" edge. As the FSW tool rotates, it moves the workpiece plasticized material from one side to another. The advancing edge works hardest, as the speed of the tool shoulder edge relative to the aluminum workpiece is faster on the advancing side than it is on the retreating side. (Burkes, Hallinan and Shropshire, et al. 2008) 


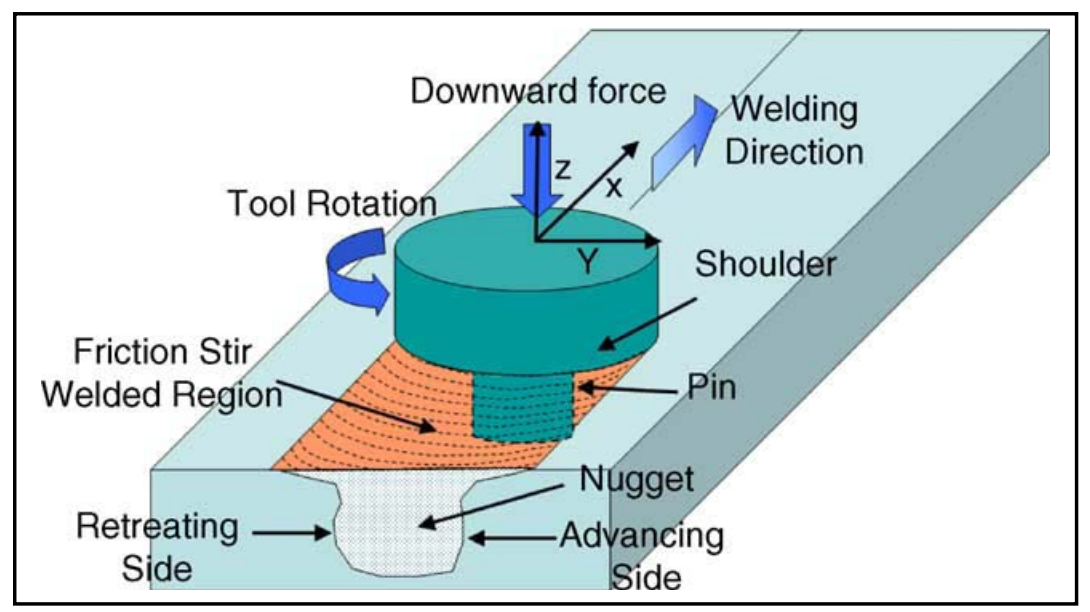

Figure 2.1 Schematic drawing of friction stir welding (Mishra and Ma 2005)

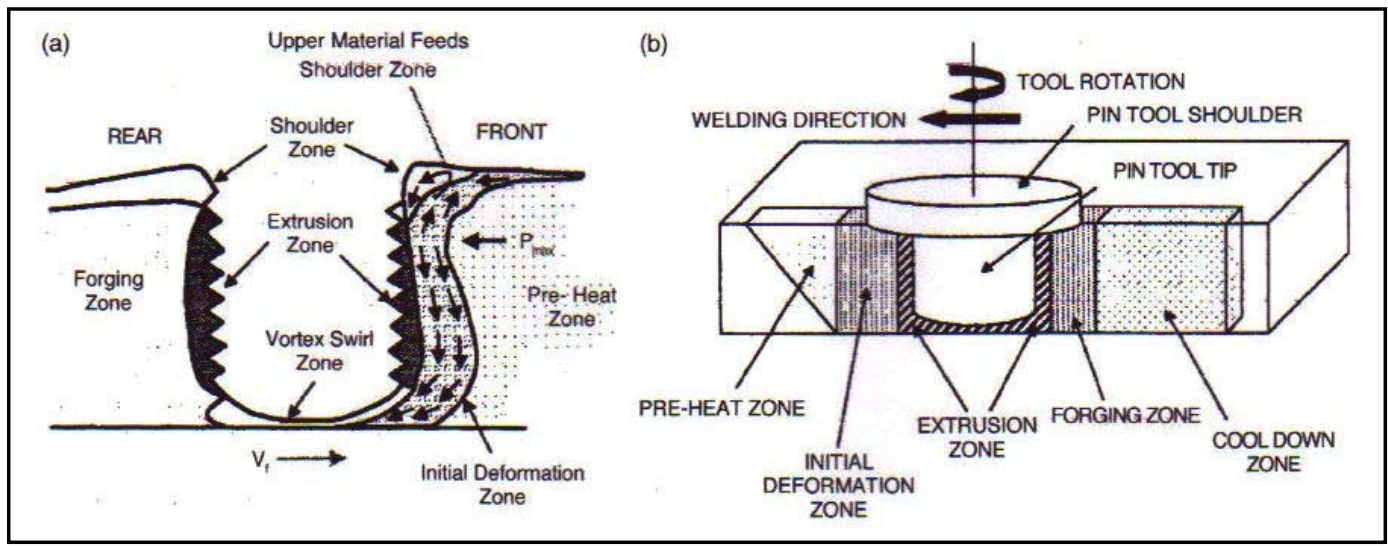

Figure 2.2 During FSW: (a) material flow patterns, and (b) processing regions [(Mishra and Ma 2005) and (W. J. Arbegast 2003)]. (Note that the two diagrams show opposite FSW directions.)

At no time during the solid-state FSW process does the joint reach a liquid state. ${ }^{5}$ In contrast to conventional (fusion) welding processes, FSW therefore results in much lower distortion and residual stresses owing to the low heat input characteristic of the process. Dimensional stability of the assembled structure is maintained, and the joint itself can have mechanical properties more compatible with the base material than those obtained using conventional welding methods. [(Marzoli, et al. 2005) and (Jin, et al. 2001)]

FSW can even join dissimilar and thermally unweldable metals. The FSW between two such materials shows a chaotic and intercalated blend so thoroughly mixed it is actually alloy at many locations. [(Marzoli, et al. 2005) and (Murr, et al. 1998)]

FSW was invented and patented by TWI, Ltd. in 1991 (Thomas, et al. 1991), and was implemented in U.S. aerospace and automotive markets in about 1995 (W. J. Arbegast 2007). The simplicity and applicability of FSW to even dissimilar materials poses a wide range of

\footnotetext{
${ }^{5}$ Aluminum Al6061-T6 has solidus temperature of $582^{\circ} \mathrm{C}$, and liquidus temperature of $652^{\circ} \mathrm{C}$ (St-Georges, et al 2007).
} 
manufacturing prospects. (Liu, et al. 1997) However, its use has not significantly spread to other industrial applications, and its industrial implementation in the U.S. is therefore lagging behind that of overseas industries. Barriers to more extensive FSW implementation in the U.S. include (W. J. Arbegast 2007):

- Lack of industry standards and specifications

- Lack of accepted design guidelines and allowable limits

- Lack of an informed workforce

- The high cost of capital equipment relative to standard welding techniques.

In 1998 the AWS D17J Subcommittee began development of a draft FSW specification (AWS No. D17.3:200X) to help address these issues. Acceptance and release is anticipated in the near term. In the meantime, most FSW users have developed internal specification for application to their products. (W. J. Arbegast 2007)

Although industrial implementation of FSW is slow in the U.S., significant research efforts are ongoing. From 1995 through 2006 over 50 U.S. patents were issued in FSW. Pin tool designs have evolved from those originally developed by TWI to unique designs for thick section, lap joint, high temperature, and fast travel speed joining. Some research efforts include (W. J. Arbegast 2007):

- In 2005, DKSS-GmbH (a German company) reported successful FSW at welding speeds in excess of $20 \mathrm{~m} / \mathrm{min}(780 \mathrm{inch} / \mathrm{min})$ in thin gauge Al butt joints.

- UofMo-Columbia is evaluating Electrically Enhanced Friction Stir Welding (EEFSW) where additional heat is applied by resistance heating through the pin tool

- UofWisconsin is developing Laser Assisted FSW (LAFSW) of aluminum lap joints where a laser is trained ahead of the pin tool to preheat the material

- Under a collaborative research program between the Army Research Lab (ARL) and the SDSMT Advanced Materials Processing \& Joining Center (AMP), a variety of FSW technologies are being developed, including complex curvature FSW, dissimilar alloy FSW, and low cost fixturing and tooling

- Interactive databases of FSW properties and processing parameters is under development by the Center for Friction Stir Processing (CFSP), which was established in 2004 with the mission of performing research, developing design guidelines and allowable limits, training, and transferring the FSW technology to a broader industrial base.

A newly developed variation of the FSW method is friction stir processing (FSP), wherein a short-pin or pinless non-consumable rotating tool is pressed onto a work piece surface and traversed for some desired length as is done in FSW. The pressure and friction from the rotating FSP tool induces intense local shear deformation and heating in the material surface, resulting in refinement of its microstructure. The microstructural modifications provided by FSP may be desirable for enhancement of mechanical properties or homogenization of precipitates in various aluminum alloys and composites. [(McNelley, et al. 2007), (Mishra and Ma 2005), and (Woo, et al. 2007)]

Tool design has been a most competitive and undisclosed area of FSW and FSP, which has made it difficult to compare data generated from the different tool designs. It is apparent, however, that small changes in tool design and process parameters result in significant changes in aluminum weld characteristics such as weld material grain growth and corrosion properties. [(Lumsden, Pollock and Mahoney 2005), (Nelson 2005), and (Mishra and Ma 2005)] No tool material other than steel was mentioned in the references, and no information was found regarding research with varying tool materials. 


\subsubsection{Microstructure after Friction Stir Welding}

\section{a. Microstructure Zones}

[(Sato, Kokawa, et al. 2001),(Sato, Urata and Kokawa 2002), (Liu, et al. 2004), (Mishra and Ma 2005), (Lumsden, Pollock and Mahoney 2005), (Pao, et al. 2005), (W. J. Arbegast 2005), (Marzoli, et al. 2005), (Cai, et al. 2005), and (Woo, et al. 2007)]

Several microstructural zones are evident in material subjected to FSW (see Figure 2.3). Through the length of its traverse, the FSW process typically creates a nugget zone with very fine grains resulting from dynamic recrystallization. In the plane perpendicular to the FSW path, nugget zone grains appear equiaxed. Depending on operational parameters, tool geometry, temperatures, and work piece materials, various nugget zone shapes have been observed and can be classified into two types: an elliptical-shaped nugget, and a basin-shaped nugget that widens near the upper surface. With the same tool geometry, different nugget zone shapes can be produced by changing processing parameters.

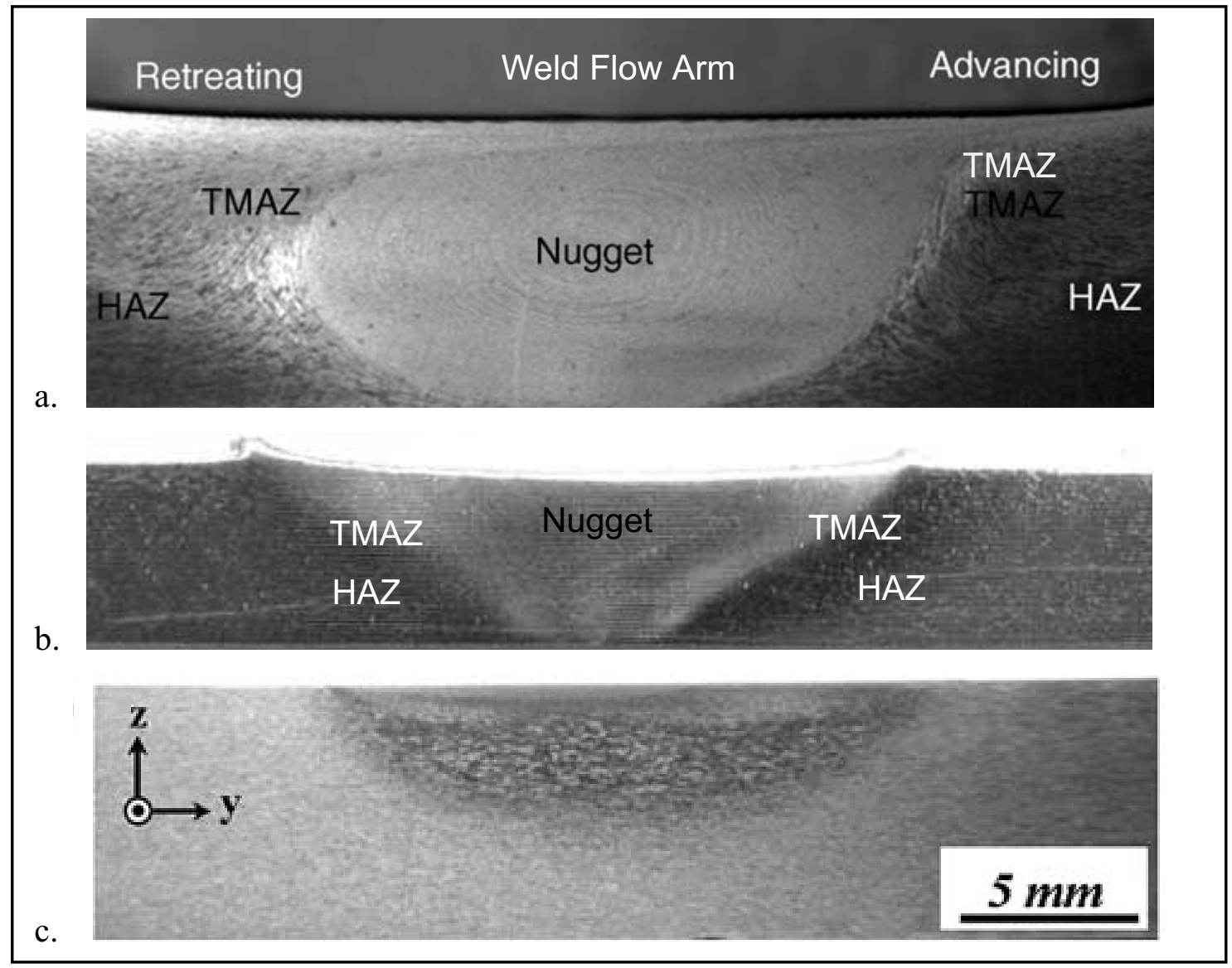

Figure 2.3 Sample cross-sections showing microstructural zones in weld regions.

a. Elliptical-shaped nugget zone in A17075-T651, created by FSW with 400 RPM, $51 \mathrm{~mm} / \mathrm{min}$ [after (Mishra and Ma 2005)]; b. Basin-shaped nugget zone in Al6063-T5, created by FSW with $800 \mathrm{RPM}, 360 \mathrm{~mm} / \mathrm{min}$ [after (Sato, Urata and Kokawa 2002)]; c. Weld region in Al6061-T6, created by FSP with pinless tool at 1250 RPM, $280 \mathrm{~mm} / \mathrm{min}$ (Woo, et al. 2007). 
Immediately outside the nugget zone is the thermo-mechanical affected zone (TMAZ), where friction heating and plastic flow during FSW creates a combination of deformed grains, recovered grains, and fine recrystallized grains, including subgrains containing a low density of dislocations. The grains are oriented in the direction of plastic deformation, around the nugget zone. Another zone that is sometimes present is the "flow-arm" region, which exists at the surface where material is dragged across the top of an elliptical-shaped nugget zone by the back side of the tool shoulder, i.e., after the pin has formed the weld and as the tool moves past. If this zone is present, its microstructure appears similar to that of the TMAZ, but with very fine grain size.

Outside the TMAZ is the heat affected zone (HAZ), where material is heated but not mechanically deformed. Here the grain structure is not disturbed and resembles base metal, although it can contain coarser precipitates and lower dislocation density. These microstructure changes can significantly alter the weld region's mechanical, fatigue, and corrosion properties. Differences are often apparent between zone locations on either side (advancing vs. retreating) of the nugget zone.

\section{b. Grain Sizes, Shapes, and Directions}

Base material of plate A16061-T6 typically has grains of about $100 \mu \mathrm{m}$ average diameter, elongated to about $200 \mu \mathrm{m}$ in the direction of the plate's rolled fabrication, and about $25 \mu \mathrm{m}$ diameter perpendicular to that [(Liu, et al. 1997), (Woo, et al. 2007), and (Chen 2007)]. After FSW, the entire weld region has grains with much different characteristics (always smaller) than grains in the base material, although the ranges of these characteristics can vary considerably depending on the aluminum composition, the FSW operational parameters, and the FSW zone.

The different FSW zones are easily distinguished by differences in grain characteristics such as size, shape, and direction, although various studies report quite a range of characteristics. Table 2.1 lists grain characteristics typical for the various zones.

Table 2.1 Typical grain characteristics in various zones of a FSW weld region.

\begin{tabular}{|c|c|c|c|}
\hline FSW Zone & $\begin{array}{c}\text { Avg. Grain } \\
\text { Diameter } \\
(\mu \mathrm{m})\end{array}$ & $\begin{array}{c}\text { Grain Shape } \\
\text { in Sample Plane }\end{array}$ & Grain Direction \\
\hline $\begin{array}{l}\text { Base Material } \\
\text { (e.g. Al6061-T6) }\end{array}$ & 25 & Equiaxed $^{\mathrm{b}}$ & None ${ }^{b}$ \\
\hline Nugget Zone & $1-10$ & Equiaxed $^{\mathrm{a}}$ & None $^{a}$ \\
\hline TMAZ \& Weld Arm & $5-20$ & Elongated & Around nugget zone \\
\hline HAZ & 25 & Equiaxed $^{b}$ & None ${ }^{b}$ \\
\hline \multicolumn{4}{|c|}{$\begin{array}{l}\text { a. FSW nugget zone grains typically appear equiaxed in the plane of the cross-section. However, grains in the } \\
\text { FSW region, including the nugget zone, are in fact elongated in the direction of the workpiece length, i.e. } \\
\text { with their direction closely associated with the direction of deformation and strain (Cai, et al. 2005). } \\
\text { b. Grains in the base material plate are elongated in the rolling direction. The rolling direction is usually } \\
\text { perpendicular to the sample plane, so base material grains appear equiaxed in the sample plane; otherwise } \\
\text { they appear elongated. HAZ grains have the same shape and direction as base material grains. }\end{array}$} \\
\hline
\end{tabular}


If a weld arm zone is not present, then grains near the top of the FSW region are usually larger sized than grains in the TMAZ or the nugget zone center because temperatures are highest at the surface, promoting more grain growth. Grain size typically decreases with distance on either side of the weld centerline and further away from the surface from top to bottom, corresponding roughly to temperature variations within the entire weld region.

Independent of the base material size and morphology, the nugget zone grains are very fine, approaching subgrain size due to dynamic recystallization. With increasing plate thickness, the temperature difference between the top and bottom of the weld increases, resulting in increased difference in grain size. The grains are typically smallest at the bottom of the FSW nugget zone. At the weld's edge, grains are larger, of uneven size and orientation, with the mid-plate's maximum grain size found in the HAZ of the advancing side. [(Benavides, et al. 1999), (Sato, Kokawa, et al. 2001), (Liu, et al. 2004), (Cai, et al. 2005), (Huneau, et al. 2005), (Mishra and Ma 2005), (Nelson 2005), (Chen 2007), and (Querin, Davis and Schneider 2007)]

\section{c. Grain Precipitates}

The FSW's rapid thermal cycle creates complex precipitate phenomena in the FSW region. In general, there are fewer and larger precipitates in the FSW region than are in the parent material, but they are of more varying types and distributions. Frictional heating from the tool shoulder drives dissolution of strengthening needle-shaped precipitates ( $\left.\beta^{\prime \prime}\right)$ from the aluminum nugget zone and TMAZ, which causes these zones to soften. During subsequent weeks the precipitates reform and some hardness is regained; this process is termed "natural aging." On the other hand, softening in the HAZ is caused by dissolution of the needle-shaped $\beta$ " precipitates and growth of rod-shaped $\beta^{\prime}$ precipitates, and is not followed by increased hardness via natural aging. [(Liu, et al. 1997) and (Woo, et al. 2007)]

(Sato, Urata and Kokawa 2002) observed that their sample made with the lowest RPM had the smallest grains and the least amount of post-FSW hardness increase due to natural aging. It was speculated that the low amount of natural aging was due to the high volume of "precipitate free zone" in the small grains relative to larger sized grains.

Depending on tool design, the nucleation and coarsening of precipitates during FSW can produce a sensitized microstructure in high-strength Al alloys. In some alloys, the sensitized microstructure is more susceptible than the parent alloy to intergranular corrosion, stress corrosion cracking, and pitting corrosion. (Lumsden, Pollock and Mahoney 2005)

\section{d. Hardness Measurements}

Base material of plate Al6061-T6 has hardness of 85 to $110 \mathrm{H}_{\mathrm{V}}$ [(Liu, et al. 1997) and (Jin, et al. 2001)], typically reported at $107 \mathrm{H}_{\mathrm{V}}$ [(Keiser, Jue and Burkes 2007) and (ASM 1990)]. After FSW, the entire weld region is softer than the base material even though its grains are considerably smaller and generally more equiaxed. ${ }^{6}$ For instance, (Liu, et al. 1997) measured hardness soon after FSW on Al6061-T6 at Hv $=55$ (near the top of the weld region) to 65 (near the bottom), with grains of about $10 \mu \mathrm{m}$. This phenomenon of reduced hardness is typical of plastic deformation and dynamic recrystallization, as the shear strain imposed by the FSW process reduces dislocation density in the material structure. In addition, of equal or greater impact depending on the aluminum composition, is that after FSW there are generally fewer and larger precipitates in the entire weld region than in the base material. (Liu, et al. 1997)

\footnotetext{
${ }^{6}$ The softer material caused by FSW is in contrast with typical material characteristics; usually materials with smaller, equiaxed grains are harder.
} 
Precipitation-hardenable Al alloys (e.g., the Al6000 series) have hardness most impacted by frictional heating during FSW, because the volume fraction, size, and distribution of their strengthening precipitates are remarkably influenced by thermal effects (Sato, Kokawa, et al. 2001).

Studies of 5000-series aluminum give examples of variations in hardness across the entire weld region: (Chen 2007) found the TMAZ hardness to be $16-30 \%$ greater than that in the nugget zone, due to more FSW work strain and grain refining in the nugget zone. Hardness was higher near top and bottom surfaces than in the center, attributed to higher magnesium precipitates in these regions than the mid-sections. Hardness fluctuations in the nugget zone and TMAZ were attributed to local variation in $\mathrm{Mg}$ content resulting from material transport during FSW process. Similarly, (Pao, et al. 2005) found substantial decreases in dislocation density in the HAZ and the nugget zone, with uniformly lower hardness in those regions.

In fact, hardness profiles across the friction stir weld often reveal that the strength is not homogeneous and reduces to a minimum in the HAZ. Three-dimensional heat flow and kinetics modeling of re-solution and subsequent re-precipitation supports this understanding of the HAZ phenomenon. (Jin, et al. 2001)

Natural aging is the precipitation-hardening phenomenon that occurs at room temperature after solution-heat-treatment processing. (Woo, et al. 2007) The extent of this phenomenon apparently depends on both the aluminum composition and the FSW processing parameters. In 7000-series aluminum, complete hardness recovery is typical: for example, (Marzoli, et al. 2005) reports that within one week after FSW, the hardness of A17005 had recovered to only slightly less than that of the base material, and within one month the hardness was completely recovered. However, (Woo, et al. 2007) reports that, after FSP on Al6061 samples, aging yielded increased hardness (but not full recovery) of TMAZ and the nugget zone due to reprecipitation, while the HAZ hardness remained unchanged.

Other studies also demonstrate the relationship between heat, precipitates, and hardness: [(Sato, Kokawa, et al. 2001) and (Sato, Urata and Kokawa 2002)] found that aging by postFSW heat treatment raised the hardness in Al6063 samples to roughly the same hardness as the base material. The hardness increase due to aging was less in samples that had been created with low-RPM FSW, probably due to relatively large precipitate-free volumes in those samples' small-sized grains. These results help confirm that hardness depends strongly on precipitate distribution, and slightly on grain size.

(Woo, et al. 2007) found that softening occurred even when FSP was performed with no pin. This supports the theory that frictional heating alone is sufficient to cause microstructural softening, without the severe plastic deformation (and additional heating) by the stirring pin during FSW. It is interesting to note that on samples made with pinless FSP, i.e. low deformation, natural aging occurred only near the top surface; when FSP was performed with a small tool pin, a more homogeneous natural aging process occurred throughout the thickness of the FSP plate.

Studies show that post-FSW heat treatment (annealing) substantially homogenizes FSW grain size and hardness, and improves mechanical properties of material in the FSW region [(Querin, Davis and Schneider 2007) and (Bhanumurthy, Kumbhar and Sharma 2007)].

\section{e. Strength and Formability}

Many studies indicate that strength of the FSW region is comparable to or even greater than that of the base material. (Marzoli, et al. 2005) reports that in tensile tests, FSW 
specimens behaved the same as the base material, demonstrating a joint efficiency of about $100 \%$. And (Chen 2007) reports that in tensile tests, all samples failed in the base material rather than in the FSW, except where there was a severe weld undercut notch.

In addition to being stronger in tensile tests, FSW and FSP specimens were also much more flexible than the base material specimens in bending tests; both Al-7075 and Al-6061 plates were bent without cracking well beyond what can be achieved without FSP (Mahoney, et al. 2005) and (Marzoli, et al. 2005)]. This makes sense, as the fine, soft grains in the FSW region should be beneficial to the weld ductility. (Chen 2007)

\section{f. Onion Rings}

Contrasting bands are commonly observed within the nugget zone of FSW cross-sections (see Figure 2.3.a); these bands are termed "onion rings" due to their concentric appearance. Although the mechanism resulting in the formation of these rings is unclear, they apparently result from heat generation and material movement during FSW. Onion rings seem to be formed by the combined rotation and forward motion of the tool; their spacing is dependent on the amount of forward movement of the tool in one rotation. During FSW the tool extrudes metal around to the retreating side. As the tool's RPM is increased, the rings' sharpness fades on the advancing side and increases on the retreating side of the nugget zone. As the tool's traverse speed is increased, the onion rings vanish, even when the ratio of the rotational speed to the traverse speed is held constant [(Querin, Davis and Schneider 2007), (Marzoli, et al. 2005), and (Krishnan 2002)]. No information was found on the impact of changed FSW tool load on onion ring formation.

Orientation imaging microscopy (OIM) examination shows that generally onion rings are apparent in all dimensions of FSW samples. The rings' complex patterns illustrate material flow patterns in intercalated vortices, both parallel and perpendicular to the tool axis. The rings' concentric appearance in typical FSW samples' cross-sections is due to a geometrical effect, like taking a section through a stack of semicylinders, with ring spacing wider at the center and narrower at the edge (see Figure 2.4). [(Krishnan 2002) and (Sato, Kokawa, et al. 2001)]

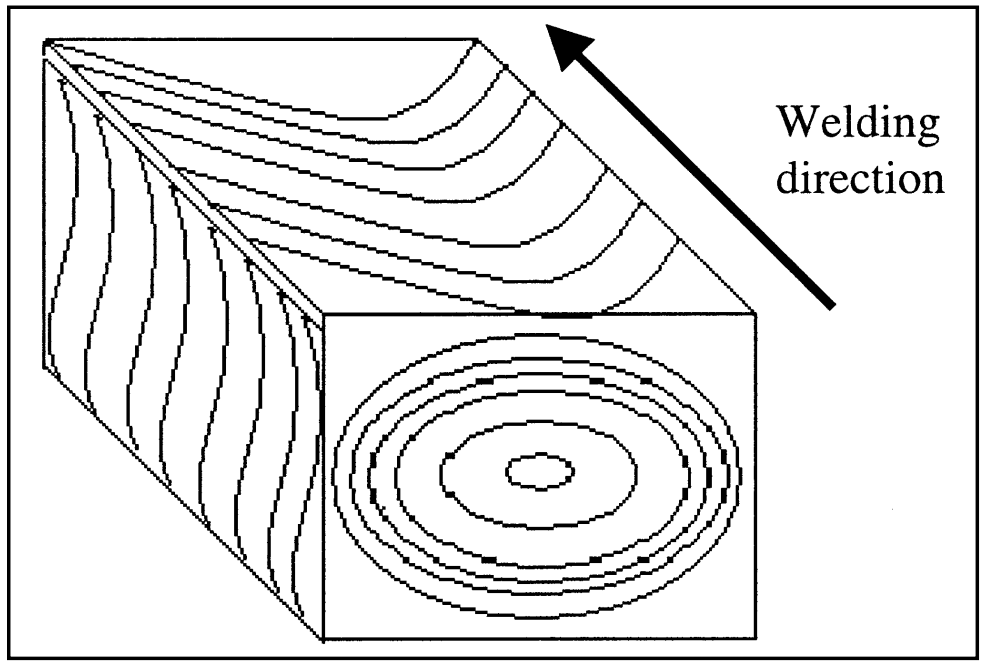

Figure 2.4 Nugget zone onion rings are apparent in all planes of FSW samples. (Krishnan 2002) 
It is thought that the rings are visually apparent due to their grain size variations and their differences in orientation of recrystallized grains [(Querin, Davis and Schneider 2007) and (Fonda, Bingert and Colligan 2004)]. Examinations show variation in grain size, hardness, and concentration of base metal impurities from one ring to the next. The rings' spacing and clarity depend on tool speed during FSW. But regardless of speed, alternating rings in FSW samples of A12024 and A12524 had grain size 10-25\% smaller, with hardness 3-7\% higher. (Yang, et al. 2004)

It is generally accepted that, however they are formed, onion rings seem to be benign, with no significance on weld properties or quality (Krishnan 2002). However, studies where flaws were deliberately embedded in FSW samples show that the initial flaw growth is strongly affected by presence of the banded microstructure (Yang, et al. 2004).

\subsubsection{Effects of Fabrication Parameters}

FSW has been described as an extrusion-die process, where the extrusion zone is located under the tool shoulder and around the pin. The volume of material flowing through the extrusion zone per revolution is a function of processing parameters, tool geometry, temperature, and material flow stress. Changes in or different combinations of these variables can result in different FSW material characteristics, at both the microstructure and macrostructure levels. (W. J. Arbegast 2005)

\section{a. Complex Process Mechanisms During Friction Stir Welding}

No single publication has comprehensively described the relationships between the different processing parameters such as tool RPM and traverse speed, and how these parameters affect key process conditions, such as temperature distribution and material conditions at the welding tool (Colligan 2007). This is because relationships between all the variables are fairly complex.

(Gould and Feng 1998) has a good description of FSW heating and plasticizing mechanisms:

1. AS FSW begins, heating between the tool and the workpiece is first defined by sliding friction.

2. Quickly, however, the contacting surfaces heat sufficiently to allow some instantaneous bonding, with subsequent shearing or tearing, characterized by a rapidly rising coefficient of friction. For FSW purposes, enhanced heat generation can be said to occur through "metallurgical coupling" between the tool and the workpiece. During this period the "friction peak" is observed, with magnitude difficult to characterize since this stage is so rapid. This stage results in considerable generation of heat, which allows material to increasingly plasticize.

3. The increasing thickness of the plastic layers then results in a final transition, during which bonding and shearing is replaced by a continuous working and recovery of the material.

During this time, the temperature profiles become relatively stable, resulting in an "equilibrium" condition.

In the first two stages of the process, aluminum is deposited onto the working surfaces of the tool. The plasticized region is then defined between this deposited material and the substrate itself. This is consistent with observations of aluminum deposits on the working surface of FSW tools. (Gould and Feng 1998) 
Multiple analytic models have been developed to help understand the effects of varying FSW fabrication parameters on heat generation and transfer, material movements, and resulting weld microstructure. Models have been correlated with empirical examinations of FSW microstructure and overall weld quality, and considerable insight has resulted. Each model has been developed to depict a specific set of FSW conditions. But because of the complex nature of heat generation and material movement during FSW, and the many variables involved, no model has been developed which describes all or even most FSW conditions. [(St-Georges, et al. 2007), (Kalya, et al. 2007), (W. J. Arbegast 2005), (Krishnan 2002), and (Gould and Feng 1998)]

(Vugrin, Schmucker and Staniek 2005) noted root flaws, i.e. areas of partial bonding, were caused by oxidized surfaces not sufficiently cleaned prior to the welding. However, other studies have found that FSW with long and threaded pins is "self-cleaning," and relatively insensitive to cleanliness of the workpiece surface (Burkes 2008).

\section{b. Fabrication Parameters' Effects on Microstructure}

The impact of the FSW process on grain characteristics is complicated because the resulting temperature, strain, and strain-rate all influence recrystallization and grain growth within the FSW region. Increased tool rotation or traverse speed increase the material strain and strain rates, causing more and faster material deformation, which results in smaller recrystallized grains in accordance with the general principles for recrystallization. Conversely, high temperatures resulting from the friction and deformation tend to promote post-FSW annealing with associated grain growth. (Benavides, et al. 1999) With increased deformation rate and higher temperatures, the material plasticizes, resulting in lower strain and strain-rate, and less heat production.

In short, drivers for FSW microstructure depend on fabrication parameters that provide different combinations of strain, strain rates, and temperature. Such fabrication parameters and their impact on these variables have been the subject of many studies in efforts to understand their effects, thresholds, and combinations that yield optimum grain characteristics. [(Gould and Feng 1998), (Benavides, et al. 1999), (Sato, Urata and Kokawa 2002), (Yang, et al. 2004),(Adams-Hughes, et al. 2005), (W. J. Arbegast 2005), (Cai, et al. 2005), (Marzoli, et al. 2005), (Mishra and Ma 2005), (Nelson 2005),(Colligan 2007), (Kalya, et al. 2007), (Oberembt, et al. 2007), (Querin, Davis and Schneider 2007), (St-Georges, et al. 2007), and (Woo, et al. 2007)]

However complex the relationships are between FSW processing parameters and resulting thermal conditions, it is known that FSW fabrication process parameters that move material and drive temperatures correlate strongly with resulting grain characteristics of the various weld region zones (Benavides, et al. 1999). Studies agree that the peak temperature of the FSW/FSP thermal cycle is the dominant factor in determining the recrystallized grain size, even more so than the degree of deformation [(Kalya, et al. 2007), (Cai, et al. 2005), and (Mishra and Ma 2005)]. Regardless of the specific heating mechanism, heating rates scale roughly with surface speed of the rotating tool. Due to its smaller diameter, pin surface speed is much less than that of the shoulder, so there is substantially less heating at the pin compared to the shoulder. FSW bonding actually occurs as the pin interacts with material pre-heated and plasticized by the shoulder. This pre-heated material is apparently subjected to continuous extrusion by the tool pin, facilitating a bond. (Gould and Feng 1998) Thus small changes in tool design and process parameters result in significant changes in weld microstructure (Nelson 
2005). In fact, even with the same tool geometry, different nugget zone shapes can be produced by changing processing parameters. (Mishra and Ma 2005).

FSW heat generation and material movement involve rapid transients and steep gradients in strain, strain rate, and temperature. Peak temperatures experienced by the workpiece material have not been reliably determined by direct measurement during experiments, although peak FSW temperatures occurring at the top of aluminum workpieces have been reported at from 250 to $480^{\circ} \mathrm{C}$, with most above $400^{\circ} \mathrm{C}$. Of course these peak temperature values vary depending on the aluminum composition, tool geometry, and processing parameters. [(McNelley, et al. 2007), (Adams-Hughes, et al. 2005), (Liu, et al. 1997), (Benavides, et al. 1999), (Sato, Urata and Kokawa 2002), and (Jin, et al. 2001)] About half-way through the plate's thickness, the maximum grain size is consistently found on the advancing side of the HAZ; therefore it is evident that different temperatures occur at the advancing and the retreating sides of the tool (Cai, et al. 2005).

As stated above, FSW peak temperature is the dominant factor in determining grain size. In addition it is commonly held that tool rotation speed and traverse rate are the key parameters that determine the temperature of the FSW process (Kalya, et al. 2007). When desired, it appears that an optimum combination of tool rotation speed and traverse rate for generating the finest grain size can be found when other variables (e.g., alloy, workpiece geometry, and tool geometry) are held constant. [(W. J. Arbegast 2005), (Cai, et al. 2005), (Marzoli, et al. 2005), (Mishra and Ma 2005), (Colligan 2007), and (Querin, Davis and Schneider 2007)]

Of the tool RPM and traverse speed parameters, the former is the stronger temperature driver. Increased tool RPM yields increased grain size, assumed due to heating from the higher speed's increased friction heat. As RPM is increased, temperature increases up to some threshold (dependent on aluminum composition and tool shoulder diameter), after which the temperature does not get much higher. [(Sato, Urata and Kokawa 2002), (Jin, et al. 2001)] Reduced tool RPM yields lower temperatures and finer grains, with associated enhanced material properties (Adams-Hughes, et al. 2005). This resulting smaller grain size is consistent with the general rules for recystallization, where recrystallized grain size decreases with the increased strain or strain-rate that occurs at reduced temperature (Benavides, et al. 1999).

FSW tool traverse rate plays a lesser role in driving the temperature; increased FSW tool traverse speed often but not always causes increased grain size for a given tool RPM (AdamsHughes, et al. 2005). In fact, a stronger correlation with weld heating is evidenced by the ratio of RPM/traverse speed than just the variable of traverse speed itself. Slow FSW, i.e., either low RPM or low ratio, results in micro-fine grains, while medium- and fast-FSW (with either high RPM or high ratio) have higher temperatures and produce much larger grain size [(Querin, Davis and Schneider 2007), (Yang, et al. 2004), and (Mishra and Ma 2005)]. A combination of both high RPM and high ratio yields "hot" welds, while low RPM and low ratio yields "cold" welds (Gould and Feng 1998).

Different FSW speeds result in different hardness of the FSW region (Sato, Urata and Kokawa 2002). Fast FSW yields the highest and most uniform nugget zone hardness, thought to be due to the combination of higher temperature and faster quenching (shorter transient) allowing some precipitate hardening capability via natural aging. Slow FSW yields lower but still uniform hardness in both the HAZ and nugget zone, with little or no natural aging. (Yang, et al. 2004) Even though the hardness values differ depending on FSW speed, the shape of hardness profiles across the FSW region are the same except for width (Sato, Urata and Kokawa 2002). 
On each side of the nugget zone, differences are apparent in grain sizes, orientation, hardness, and onion ring appearance [(Cai, et al. 2005) and (Querin, Davis and Schneider 2007)], implying that the advancing and retreating edges impart differing amounts of strain rate and heat into the material. This makes sense, as the speed of the tool shoulder edge relative to the aluminum workpiece is faster on the advancing side than it is on the retreating side. Thus the advancing edge is working harder against the aluminum than the retreating edge is. (Burkes, Hallinan and Shropshire, et al. 2008)

Not many studies have investigated the impact of the vertical tool load on microstructure. However, (W. J. Arbegast 2005) determined that the resultant force R, comprised of components in $\mathrm{x}, \mathrm{y}$, and $\mathrm{z}$ directions (due to tool rotation, transverse movement, and vertical tool load), is a good predictor or indicator of weld quality. And (Gould and Feng 1998) found agreement between empirical data and results predicted by a heat flow model where the vertical tool load had a linear effect on the calculated heating.

\section{c. Fabrication Parameters' Effects on Macrostructure}

Regardless of the specific mechanism of material heating by any combination of processing parameters, FSW is possible when thermal conditions are established that offer the potential for plasticity through the depth of the workpiece thickness. (Gould and Feng 1998) investigated the extreme ends of the range of thermal conditions during "hot" and "cold" FSW. It was observed that if overheating and melting occur at the workpiece surface, thin layers of aluminum are extruded from the surface, causing material expulsion and flash formation. On the other hand, the effects of insufficient heating during cold FSW were readily apparent by void formation and a running pore (i.e., lack of bonding) along the bond line. This occurs because of an insufficient amount of plasticized material around the tool pin. [(Gould and Feng 1998) and (W. J. Arbegast 2005)]

But when processing parameters are selected that create thermal conditions between these two extremes, then successful FSW can occur. Processing parameters can be determined to attain both desired microstructure characteristics and to optimize desired macro-scale performance (W. J. Arbegast 2005). In fact, (Marzoli, et al. 2005) concludes that since highquality welds can be obtained for a wide range of FSW parameter combinations, the FSW process has the robustness required for industrial applications.

\subsection{RERTR Program's Monolithic Fuel and FB Fabrication}

Friction stir welding (FSW) or friction stir processing (FSP) simply hasn't been done the way it's being done now at the INL. Therefore a new term - friction bonding (FB) - has been adopted for the INL's variant process. Because FB is currently under development at the INL and not yet performed anywhere else, literature discussing FB has been generated primarily at the INL. This section discusses the state of the art of the RERTR fuel development program and its irradiation experiments, monolithic fuel fabrication methods, the FB process and related studies, and findings of monolithic fuel examinations. Additional relevant information regarding uranium fuel phase structures and interface chemical reaction layers is available in Appendix A.

\subsubsection{Overview of RERTR Fuel Development Program}

The goal of the RERTR advanced fuel development program is to complete the conversion of all targeted research reactors by 2014. (DeMint, et al. 2007) In order to convert a reactor 
using HEU to one using uranium of lower enrichment, the amount of ${ }^{235} \mathrm{U}$ must be about the same after the conversion. This requires the new fuel, made of uranium with lower percent of ${ }^{235} \mathrm{U}$, to have substantially higher total uranium. Any reactor fuel conversions must be done without significant penalty in reactor performance or safety and involve, at most, minimal geometry changes in the fuel element or core design. (Clark and Briggs 2004) Two fuel designs are currently under development by the RERTR program: "dispersion" fuel and "monolithic" fuel.

Dispersion fuels have been an important focus area for RERTR fuel development since the mid-1990's, and much has been learned and incorporated into their design. Some aspects of dispersion fuel development and examination results are important to understanding fabrication, irradiation performance, and examination results of monolithic fuel, especially regarding uranium-aluminum interaction and behavior of alloy phases: ${ }^{7,8}$

- When the assembly is subjected to high temperatures for a time, chemical interaction occurs at the interface between the uranium and aluminum. This interface reaction layer causes the fuel to swell, and reduces heat transfer out of the fuel during irradiation. The resulting higher temperatures promote the chemical reaction and the layer formation, thus exacerbating the adverse cycle. Alloying the $\mathrm{Al}$ with $\mathrm{Si}$, and the $\mathrm{U}$ with $\mathrm{Mo}$, helps to reduce formation of this reaction layer.

- During irradiation, gas fission products are created within the uranium fuel, causing the fuel to swell even more. Increased irradiation yields more such fission products. These tend to form into minute bubbles that migrate toward the aluminum-uranium interface layer. There they coalesce into larger bubbles that weaken the bond and reduce heat transfer out of the fuel. Maintaining the uranium in $\gamma$-phase helps to reduce bubble formation and migration, and adding $\mathrm{Si}$ to the $\mathrm{Al}$ and $\mathrm{Mo}$ to $\mathrm{U}$ helps reduce bubble coalescence at the interface layer.

- Irradiation and elevated temperatures cause phase changes in the uranium alloy that decrease its effectiveness in blocking bubble formation and migration.

- Elevated temperatures also cause hardening precipitates normally found in the Al6061T6 to dissolve into the aluminum matrix, leaving the aluminum softer, more ductile, and less strong. In addition, aluminum precipitates migrate into the uranium fuel matrix (Keiser, Jue and Clark 2006). It is as yet unclear what effect, if any, these precipitates have in the uranium, but so far foils with aluminum precipitates in them exhibit good irradiation performance (Keiser, Robinson and Finlay 2007).

Although it is more challenging to fabricate, monolithic fuel has several advantages relative to dispersion fuel. The fuel alloy foil allows uranium densities far in excess of those found in dispersion fuel. Thus it may be the only path to converting many of the high power reactors currently targeted by the GTRI; of 106 reactors currently identified for conversion to LEU, it is expected that 28 require monolithic fuel [(Wachs, Ambrosek, et al. Oct 2006a) and (DeMint, et al. 2007)]. Another advantage is that the single-piece monolithic fuel foil also contains a significantly lower interface area between the fuel and the aluminum in the plate, reducing the total surface area of the fuel available to react with aluminum and thus limiting the amount of detrimental uranium-aluminum interaction that can occur. In addition,

\footnotetext{
${ }^{7}$ Topics of uranium-aluminum interface reaction and behavior of uranium-molybdenum alloy phases are discussed in more detail in Appendix A.

${ }^{8}$ Information is summarized from (Clark, Meyer and Hayes 2002), (Clark and Briggs 2004), (Wachs, Ambrosek, et al. Oct 2006a), (Clark, Moore, et al. 2007), (D. M. Wachs, D. D. Keiser, et al. Sept 2007), and (Kim, et al. 2007).
} 
monolithic fuel moves the interface to a cooler region of the fuel plate, where thermal conditions are less conducive to reaction layer formation. [(Clark and Briggs 2004) and (Keiser, Jue and Clark 2006)]

Understanding a fuel's performance in a reactor radiation environment is of course an essential part of the RERTR Program's fuel development efforts. Therefore multiple irradiation experiments have been conducted and are planned for the future. Each experiment has differed in its fuel design or was subjected to differing reactor conditions such as power density, temperature, length of irradiation, and percent of the fuel's ${ }^{235} \mathrm{U}$ that was fissioned (also termed "burn up" or "BU"). Information gathered from these experiments has and will determine the fuel development path.

\section{a. RERTR Program's Irradiation Experiments to Date}

All RERTR experiments irradiated in the Advanced Test Reactor (ATR) at the INL have included dispersion fuel, which is well on its way to qualification. Monolithic fuel was introduced later, and is now the focus of intense research. The following is a summary ${ }^{9}$ of the RERTR experiments to date, plus a few full-sized fuel experiments in the OSIRIS reactor in France:

- RERTR-1\&2: In 1997-1998, dispersion fuel was irradiated in the ATR at low power density and temperature, with peak fuel temp $<100^{\circ} \mathrm{C}$. RERTR-1 $=\sim 40 \%$ BU; RERTR-2 $=\sim 70 \% \mathrm{BU}$. Fission gas bubble formation was found to be inversely proportional to the Mo content of the fuel.

- RERTR-3: In 1999, dispersion fuel was irradiated in the ATR at high power density and temperature, to $\sim 40 \% \mathrm{BU}$. There was greater than expected fuel-matrix interaction, with amounts inversely proportional to the Mo content of the fuel.

- RERTR-4\&5: In 2001-2002, dispersion and monolithic fuels were irradiated in the ATR, with RERTR-4 experiencing moderate power density and temperature to $\sim 80 \% \mathrm{BU}$, and RERTR-5 experiencing moderately high power density and temperature, to $\sim 50 \% \mathrm{BU}$. The two monolithic fuel plates in RERTR-4 were fabricated by roll bonding U10Mo foil in Al6061 cladding. The foils were $12 \mathrm{~mm}$ diameter disks, $0.3 \mathrm{~mm}$ thick, which broke into pieces during the roll bonding process.

- RERTR-6: In 2005, dispersion and monolithic fuels were irradiated in the ATR at moderate power density and temperature, to $\sim 45 \% \mathrm{BU}$. This was the first irradiation of mini-plate monolithic fuel; contained ten monolithic mini-plates, all fabricated by FB, with various percentages of alloyed Mo. Promising overall performance of monolithic fuel.

- RERTR-7: In 2005-2006, dispersion and monolithic fuels were irradiated in the ATR at moderately high power density and temperature, to $\sim 80 \% \mathrm{BU}$. Contained monolithic mini-plates (fabricated by TLPB and FB) with various percentages of alloyed Mo. The TLPB plates cracked and one released fuel particles into the reactor during operation. PIE was completed in 2007.

\footnotetext{
${ }^{9}$ Information is summarized from (Clark, Meyer and Hayes 2002), (Clark and Briggs 2004), (Jarousse, Lemoine, et al. 2006), (Wachs, Ambrosek, et al. Oct 2006a), (Finlay, Wachs and Hofman 2006), (Clark, Jue, et al. October 2006), (Burkes, Hallinan and Clark, et al. January 2007b), (Clark, Moore, et al. 2007), (D. M. Wachs, D. D. Keiser, et al. Sept 2007), (Finlay, Wachs and Robinson, et al. 2007), (Burkes, Hallinan and Wight, et al. September 2007), (Jarousse, Lemoine, et al. 2007), and (Wachs April 2007).
} 
- RERTR-8: In 2006-2007, dispersion and monolithic fuels were irradiated in the ATR at moderately high power density and temperature, to $\sim 75 \%$ BU. Contained monolithic mini-plates (fabricated by HIP and FB), with a range of Mo content, and one plate with a layer of $\mathrm{BAl}$ attached to the foil to demonstrate incorporation of burnable poison. Irradiation was completed in February 2007, and PIE is underway.

- RERTR-9: In 2007, dispersion and monolithic fuels were irradiated in the ATR at moderately high power density and temperature to $75 \%$ BU. Contained monolithic miniplates (fabricated by HIP and FB with various fabrication parameters and configurations), with a range of Mo content and different types of diffusion barriers. Irradiation was completed in 2007, and PIE is underway.

- IRIS-3: In 2005, full-sized dispersion fuel was irradiated in the OSIRIS reactor at moderate power density and temperature. Fuel with $0.3 \%$ Si matrix material was removed ahead of schedule due to excessive swelling at $\sim 38 \%$ BU. Fuel with $2.1 \% \mathrm{Si}$ matrix material performed well and completed the test cycle, to $\sim 48 \% \mathrm{BU}$. PIE is currently underway.

- IRIS-TUM: In 2005-2006, full-sized dispersion fuel was irradiated in the OSIRIS reactor at moderate power density and temperature. Fuel with both $0.3 \% \mathrm{Si}$ and $2.1 \% \mathrm{Si}$ matrix materials seemed to perform well and completed the test cycle (fuel particles were fabricated by grinding rather than atomization). PIE is underway.

\section{b. RERTR Program's Plans for Future Irradiation Experiments}

The RERTR program fuel development effort is still faced with challenges on several levels. Working within budget and schedule constraints, the program's technical challenges include providing fuels of varying shapes and sizes as required by various reactors, assuring safe encapsulation of the fuel with good irradiation and thermal performance, identifying materials and fabrication techniques that best support these efforts, and providing fuel performance validation to support NRC review and qualification of the fuel. Future plans for fuel fabrication tests and examinations will help move the program toward achieving these goals. Plans are to fabricate fuel plates of multiple sizes and designs, using various fabrication techniques, parameters, equipment, and materials; perform characterization via ultrasound, mechanical testing, microstructural examinations, modeling, and post-irradiation examination; correlate pre-irradiation \& post-irradiation observations; and better understand relationships between processes, properties, and microstructure. The final selected fuel designs and validation data will be submitted to the NRC in late 2010, starting the qualification and approval process to allow the fuels' use in research reactor operation. [(Burkes, Keiser and Miller, et al. January 2007a), (D. M. Wachs, D. D. Keiser, et al. Sept 2007), and (D. M. Wachs, D. D. Keiser, et al. 2008)]

A key part of the fuel development is irradiation testing on fuels which explore various design and fabrication aspects such as: larger (half-sized and full-sized) plates; plates fabricated with different equipment, at different facilities, or with new tooling designs; and fuels that incorporate materials such as $\mathrm{Mo}, \mathrm{Nb}, \mathrm{Zr}$, and $\mathrm{Si}$ barriers, either co-rolled with the foil or applied as thermal sprays. The following is a summary ${ }^{10}$ of some plans for future fuel irradiation experiments:

\footnotetext{
${ }^{10}$ Information is summarized from (Jarousse, Lemoine, et al. 2006), (Wachs, Ambrosek, et al. Oct 2006a), (Clark, Jue, et al. October 2006), (D. M. Wachs, D. D. Keiser, et al. Sept 2007), (Burkes, Hallinan and Wight, et al.
} 
- RERTR-10: In 2008, monolithic fuel (both HIP and FB) will be irradiated in the ATR at high power density and temperature for one reactor cycle. Fuel features such as diffusion barriers, burnable poisons, mechanically constrained plates, and thicker foils will be investigated.

- RERTR-11: In 2009, dispersion fuel will be irradiated in the ATR with experimental features as needed to help complete the database of information on dispersion fuels.

- RERTR-12: In 2010, monolithic fuel will be irradiated in the ATR with experimental features as needed to help complete the database of information on monolithic fuels.

- AFIP-1: In 2008, full-sized (for ATR) dispersion fuel, dubbed the AFIP (스R Full-size plate In center flux trap Position) will be irradiated in the ATR at moderately high power density and temperature to $\sim 70 \% \mathrm{BU}$. The fuel has been fabricated at BWXT, Inc. in Virginia, and is currently awaiting shipment to the INL.

- AFIP-2: In 2008, ATR-full-sized monolithic fuel will be irradiated in the ATR at moderately high power density and temperature to $\sim 70 \% \mathrm{BU}$. The monolithic plates will be FB plates fabricated at INL.

- AFIP-3: In 2008, ATR-full-sized monolithic fuel, to be irradiated in the ATR at moderately high power density and temperature to $\sim 70 \%$ BU. The monolithic plates will be HIP fabricated at BWXT in Virginia, using foils produced at DOE's Y-12 facility in Tennessee.

- AFIP-4: In 2008, ATR-full-sized monolithic fuel, to be irradiated in the ATR at moderate power density and temperature. The monolithic plates will include deliberate debonds between the foil and cladding to help understand the impact of debonds on the fuel during irradiation. In addition, features such as involute shape, density zoning, and burnable poisons might be tested.

- BR2: In about 2010, full-sized dispersion and monolithic fuel plates and/or fuel element assemblies will be irradiated in the BR2 reactor in Belgium.

- IRIS-4: In 2008, full-sized dispersion fuel plate, to be irradiated in the French OSIRIS reactor. Dispersion fuel particles will be oxidized-atomized.

- IRIS-5B: In 2008-2009, full-sized monolithic fuels, most likely FB, will be irradiated in the OSIRIS reactor. Includes two FB plates from the INL, and two plates fabricated under a new, proprietary welding process to be provided under a 2005 agreement between the French governmental agency CEA (Commissariat à l'Energie Atomique), the TUM (Technical University of Munich, Germany), and the French public multinational industrial conglomerate AREVA-CERCA (AREVA-owned Compagnie pour l'Etude et la Réalisation de Combustibles Atomiques).

- Other as-yet unspecified future irradiation tests will be performed to help evaluate advanced fuel designs (e.g., foils with graded profiles, shaped fuel, and incorporation of burnable poisons) as they are developed for special purpose reactors like HFIR and ATR, and as needed to help complete the database of information on full-sized fuels and fuel element assemblies.

September 2007), (Jarousse, Lemoine, et al. 2007), (Wachs April 2007), (D. M. Wachs, D. D. Keiser, et al. 2008), and (Burkes 2008). 


\subsubsection{Monolithic Fuel Fabrication}

As explained, monolithic fuels are will make possible the conversion of research reactors to LEU fuel, especially the highest-power reactors currently targeted by the GTRI (including HFIR, ATR, MURR, MIT, and NIST in the United States). Some of these reactors will require a complex fuel shape with tailored fuel grading and the incorporation of burnable poisons. (Wachs, Ambrosek, et al. Oct 2006a) However, traditional roll-bonding techniques are inadequate for fabrication of even the basic monolithic fuel form, as the monolithic foil tends to break apart during rolling, leading to voids \& potential blister sites (Clark, Meyer and Hayes 2002). So implementation of monolithic fuel is dependent on the development of suitable fabrication methods. Goals for monolithic fuel bonding methods include (Clark and Briggs 2004):

- Impart little or no reduction into the plate assembly to avoid tearing the fuel foil

- Achieve good adhesion both in the cladding-cladding bond and in the cladding-fuel bond

- Can be scaled-up and implemented with as little complexity and capital outlay as possible, suitable as a commercially viable manufacturing method.

Several techniques for monolithic fuel fabrication have been pursued, including TLPB, HIP, and FB. Investigation of alloys and diffusion barriers are incorporated into all the fabrication techniques. The final method will be selected in early 2009 (D. M. Wachs, D. D. Keiser, et al. 2008), and might even be based on a combination of the three techniques.

\section{a. Foil Production to Prepare for Monolithic Fuel Fabrication}

Foil production operations at the INL are conducted inside an argon-atmosphere glovebox. Uranium and molybdenum are arc-melted together in the desired proportions and the mixture is poured and cast into coupons. These are rolled to make monolithic fuel foils; a number of different combinations of rolling steps have been used.

Initially, the cast coupons were cold-rolled to $90 \%$ reduction, resistance-annealed in argon at $925^{\circ} \mathrm{C}$, and sheared to size. Cross-sections of such foils are shown in Figure 2.5. But cold work stresses often caused cracks during rolling, and cracks sometimes developed later even after the annealing step. [(Clark, et al. 2003), (Clark and Briggs 2004), and (Clark, Moore, et al. 2007)]

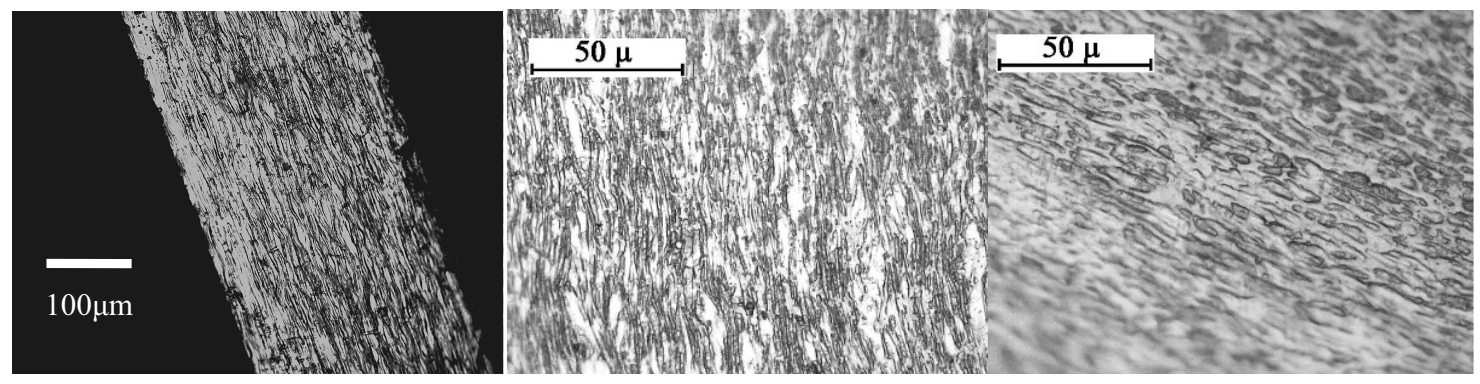

Figure 2.5 Cross-sectioned DU10Mo foils prepared at the INL

by cold-rolling to $90 \%$ reduction, resistance-annealing at $925^{\circ} \mathrm{C}$ (as measured subjectively using an optical pyrometer) and cooling to ambient temperature within a minute. Note the foils exhibit the classic elongated grain structure formed in rolled metals [(Keiser, Jue and Clark 2006), and (Clark, Hallinan, et al. May 2006)]. 
An improved practice (used to make the foils for this thesis study) is to encapsulate the coupon in a carbon steel can lined with yttria to prevent sticking, and hot rolled at $650^{\circ} \mathrm{C}$ to $85 \%$ reduction. Then it is decanned, cleaned of yttria, and cold-rolled to attain the remaining $15 \%$ reduction to the target thickness (typically $0.025 \mathrm{~cm}$ ). The final step is resistanceannealing in argon at $925^{\circ} \mathrm{C}$ for a few seconds to relieve residual stresses prior to shearing to the desired size. This foil production technique yields a success rate of over $90 \%$ for both minisized ( $8.25 \mathrm{~cm}$ long x $1.9 \mathrm{~cm}$ wide) and full-sized foils $(51 \mathrm{~cm}$ long $\mathrm{x} 4 \mathrm{~cm}$ wide). Production of a graded foil (i.e., with foil thickness changing across the width and length as is needed for some reactors, e.g., HFIR) was successfully demonstrated using this technique: a $40 \mathrm{~cm} \times 2.3$ $\mathrm{cm}$ U10Mo foil was rolled to wedge shape with thickness of $0.0068 \mathrm{~cm}$ on one edge and $0.0363 \mathrm{~cm}$ on the other. [(Clark, et al. 2003), (Burkes, Keiser and Wachs, et al. March 2007), and (Clark, Moore, et al. 2007)]

A foil-production technique that immerses as-cast ingots into a salt bath, hot rolls them to near thickness, and cold rolls them to final thickness has also been explored at the DOE's Y-12 facility in Tennessee. In addition, AREVA-CERCA and CNEA (Argentina's Comisión Nacional de Energía Atómica) are experimenting with hot-rolling UMo foils pre-clad with Al or Zr. (D. M. Wachs, D. D. Keiser, et al. Sept 2007)

In support of RERTR program goals, efforts are underway at Y-12 to develop \& validate a production-oriented, monolithic UMo fuel fabrication process adaptable for potential implementation in a manufacturing environment. As part of that effort, rolling of DUMo foils to the desired size has successfully been accomplished via hot rolling (with heat treatment) to an intermediate thickness and cold-rolling to the final thickness. Effects of carbon and other impurities in the uranium are under evaluation. Techniques are being developed to apply $\mathrm{Nb}$ and $\mathrm{Zr}$ diffusion barriers to the foils via physical vapor deposition (PVD); these will be evaluated at the INL. (DeMint, et al. 2007)

\section{b. Fuel Fabrication by Transient Liquid Phase Bonding (TLPB)}

(Clark and Briggs 2004) explains that TLPB relies on a very thin eutectic-forming interlayer foil material to diffuse into the bonding interfaces and join the materials together. By insertion of a suitable material between the cladding plates, and application of heat, a eutectic liquid phase is formed in this interface. This temporary liquid phase spreads across the interface, diffuses into the cladding and forms a metallurgical bond that joins the two plates together. TLPB with silicon was used to fabricate fuel plates irradiated in the RERTR-7A experiment. Silicon and aluminum form a eutectic at $573^{\circ} \mathrm{C}$, significantly lower than the melting point of aluminum $\left(652^{\circ} \mathrm{C}\right)$.

One TLPB method used at the INL involves thermal spray of a silicon powder plasma onto the aluminum (Burkes 2008). Another TLPB method blends silicon powder with ethanol and glycerin to facilitate application of the Si interlayer. A thin film of this mixture is applied, allowed to air dry, and then the ethanol and glycerin are "burned out" using a hot plate. The aluminum plates are assembled with the silicon powder in the interface. The assembly is loaded into a hot press and heated to $590^{\circ} \mathrm{C}$ under load. The temperature and pressure are maintained for up to 30 minutes, after which the plate is removed from the press. (Clark and Briggs 2004)

Four monolithic fuel plates were fabricated with Si TLPB and irradiated in the RERTR-7A experiment. Their uranium-aluminum interfaces showed very stable behavior prior to

irradiation. However, during irradiation two of the fuel plates cracked, and one leaked $50 \%$ of its fuel mass into the ATR coolant. [(Clark, Jue, et al. October 2006) and (D. M. Wachs, D. D. 
Keiser, et al. Sept 2007)] The crack was thought to be caused by Si embrittlement and weakening of the Al-Al bond, where significant Si-rich precipitates were observed during PIE on other RERTR-7A TLPB plates that did not fail. [(Finlay, Wachs and Robinson, et al. 2007) and (Robinson and Finlay 2007)]

No TLPB plates have been irradiated since that incident, but experiments are continuing with the use of thermal sprays in HIP and FB fuel plate assemblies (avoiding the Al-Al interface). This works well with the HIP process, but it was determined that FB temperatures are not high enough for long enough to promote the eutectic Si-Al diffusion. Therefore an AlSi spray used in a FB fuel plate assembly must be well homogenized prior to application. (Burkes, Hallinan and Wight, et al. September 2007) The AFIP-2 FB fuel plates include one made with a pure Si thermal spray (Swank, Haggard and Burkes 2008).

\section{c. Fuel Fabrication by Hot Isostatic Pressing (HIP)}

In this sold-state process, heat and pressure are applied to the process piece in an isostatic manner via a compressed gas. The applied heat and pressure act to achieve diffusion and bonding across the interface. For HIP fabrication of monolithic fuel at the INL, surface oxide removal from the bond interfaces prior to fuel plate assembly is critical. Up to six mini-plate assemblies are then encased in separate chambers in a steel can. The can is evacuated with a four-hour dwell time at $315^{\circ} \mathrm{C}$ to off-gas the fuel plates' bond surfaces, and then seal-welded. It is pressurized with argon and held at $583^{\circ} \mathrm{C}$ for 3 hours. Experiments are underway to explore HIP with lower temperatures and shorter dwell times. [(Burkes, Keiser and Wachs, et al. March 2007), (Clark and Briggs 2004), (Clark, Moore, et al. 2007), (D. M. Wachs, D. D. Keiser, et al. Sept 2007)]

Tests show that HIP is fully successful in bonding the fuel plates' aluminum cladding, with no remnants of a bond line between the aluminum at these conditions. However, significant reaction layer forms at the U-Al interface; fuel made of U7Mo is virtually consumed, while the U10Mo has a smaller but still significant reaction layer. Studies are underway to optimize HIP temperatures, pressures, and hold times, to minimize the U-Al interface reaction layer and maximize bonding. Research also continues on providing the interface with diffusion barriers such as $\mathrm{Zr}$ foils or Si thermal sprays. Three mini-sized monolithic fuel plates, one with U10Mo fuel and the other two with U12Mo fuel, were fabricated with HIP and irradiated in RERTR-8. PIE results are not yet available. [(Clark, Jue, et al. October 2006), (D. M. Wachs, D. D. Keiser, et al. Sept 2007)]

AFIP-3 will include full-sized HIP monolithic fuel plates. Because the INL HIP equipment cannot accommodate full-sized plates, HIP fabrication of full-sized plates will be performed at BWXT in Lynchburg, VA. (Jue, Clark and Nilles 2008)

\section{d. Fuel Fabrication by Friction Bonding (FB)}

When investigations began into using FSW at the INL for monolithic fuel fabrication, the term friction stir welding (FSW) was used to describe that development effort. However the term friction bonding (FB) was recently adopted for the INL process instead (Burkes, Hallinan and Wight, et al. September 2007). FB differs from traditional FSW in several ways: its goal is to physically bond - but to avoid stirring and mixing - two dissimilar materials; the FB pin is much shorter and relatively wider, and the plates are much thinner, so it does not truly form a nugget zone as does typical FSW; and the FB process is performed sequentially in overlapping passes, on both sides of the plate. Issues of concern for nuclear fuel that are not typically of concern for FSW applications include: weakness of the FB bonds, which are of a much 
different nature than welded bonds; formation and implications of a chemical reaction layer at the U-Al bond interface; and the complex shapes needed for some fuel plates. Conversely, the strength of the actual materials is not of as much concern, since they are generally strong enough to withstand dynamic in-reactor forces and contain the fuel, and obviously much stronger than the FB bond.

A conventional milling machine (shown in Figure B-2 of Appendix B) is used to fabricate monolithic FB fuel plates at the INL, although a new TTI machine (shown in Figure B-3) will be implemented soon. To prepare for the FB process, two pieces of Al6061-T6 plate material are assembled on top of each other, with a fuel foil nested in a pocket between them. This fuel plate assembly is clamped to an underlying anvil mounted on the milling machine's movable table. To perform FB, the tool (illustrated in Figure 2.6) is rotated and the pin in the center of the FB tool is plunged into the top surface of the fuel plate assembly. The pin does not extend past the top aluminum plate, but frictional heat between the wear resistant welding tool and the aluminum causes the latter to soften without reaching its melting point. This softening allows traversing of the tool through the top aluminum plate, during which plasticized material is transferred from the advancing edge of the rotating tool to the retreating edge of the tool. The plasticized aluminum forms a solid phase intermolecular bond with the layer of metal below it, forged by the downward pressure exerted from the tool. Multiple overlapping traversing passes are made until the entire plate surface has been subjected to the FB process, after which the assembly is turned over and the FB process is repeated on the opposite side. This assures the foil and cladding are well bonded on both sides, and also that material and grain properties are symmetrical across the fuel plate cross-section. The raster pattern typically used for the overlapping FB passes is shown in Figure 2.7. The FB process typically leaves a scalloped finish on the surface of the plate due to aluminum movement from the advancing to the retreating side of the tool. This is unsuitable for reactor use without further processing, so this surface is smoothed and the plate is reduced to final thickness by mechanical polishing. [(Clark and Briggs 2004), (Keiser, Jue and Clark 2006),(Clark, Hallinan, et al. May 2006),(Burkes, Keiser and Miller, et al. January 2007a), (Burkes, Hallinan and Clark, et al. January 2007b), (Clark, Moore, et al. 2007), (Burkes, Keiser and Wachs, et al. March 2007), (D. M. Wachs, D. D. Keiser, et al. Sept 2007), and (Burkes, Hallinan and Wight, et al. September 2007)]

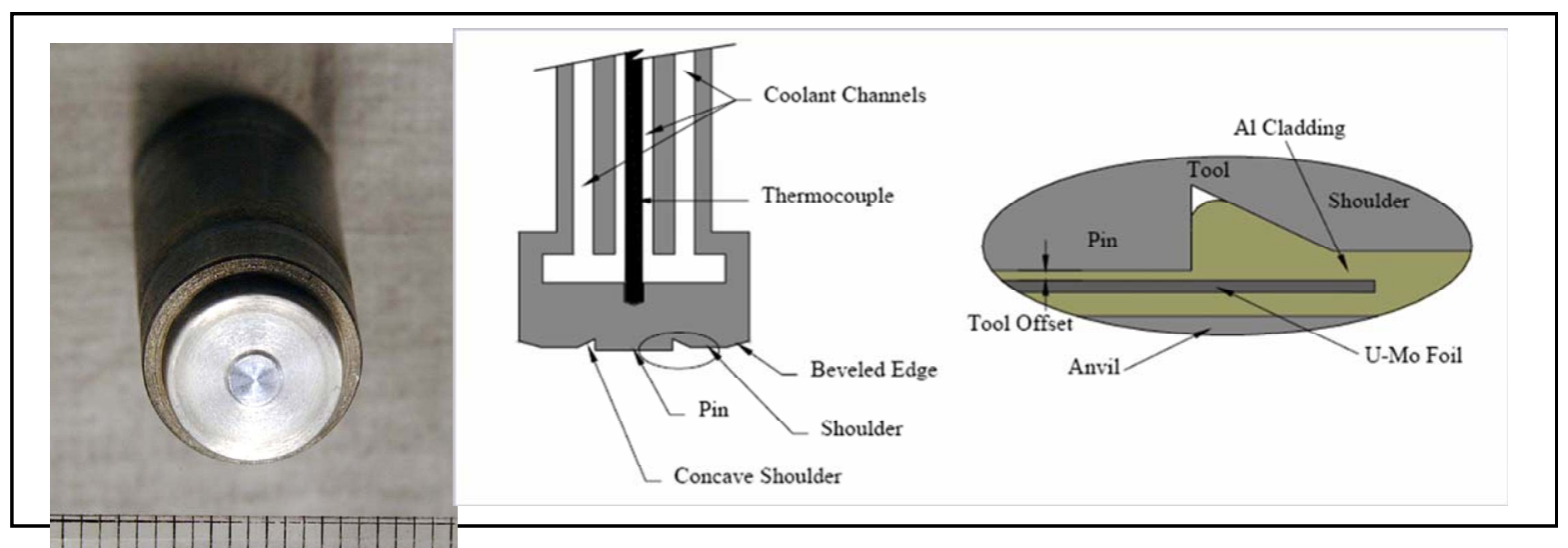

Figure 2.6 Bottom face of the FB tool. The tool interior is cooled with ethylene glycol during FB operations, and the thermocouple in the tool face interior is bathed in the coolant. The tool's pin extends slightly beyond the shoulder, which has beveled edges.

Shoulder $=3.81 \mathrm{~cm}(1.50 \mathrm{in})$ diameter, $0.714 \mathrm{~cm}(0.281 \mathrm{in})$ high. $P$ in $=1.59 \mathrm{~cm}(0.626 \mathrm{in})$ diameter, $0.0381 \mathrm{~cm}$ (0.0150 in) long. [(Clark, et al. 2003), (Clark, Hallinan, et al. May 2006), and (Dixon, Burkes and Medvedev 2007)] 
At the beginning of each FB pass, an increase in the tool's downward load is necessary as the rotating tool is first plunged into the elastic aluminum. The tool and aluminum temperatures quickly increase, and as the aluminum becomes plastic, the tool load stabilizes. (Burkes, Hallinan and Clark, et al. January 2007b) Early in FB process development efforts, it became apparent that FB with the initial tool design yielded too-high aluminum temperatures and unsuccessful fuel plate FB. So the new tool design shown in Figure 2.6 and the cooling system shown in Figure B-2.c in Appendix B were implemented during 2006, allowing a cooling flow of ethylene glycol to remove heat from the tool and anvil during the FB process. [(Clark, Moore, et al. 2007), (Clark, Hallinan, et al. May 2006), and (Dixon, Burkes and Medvedev 2007)]

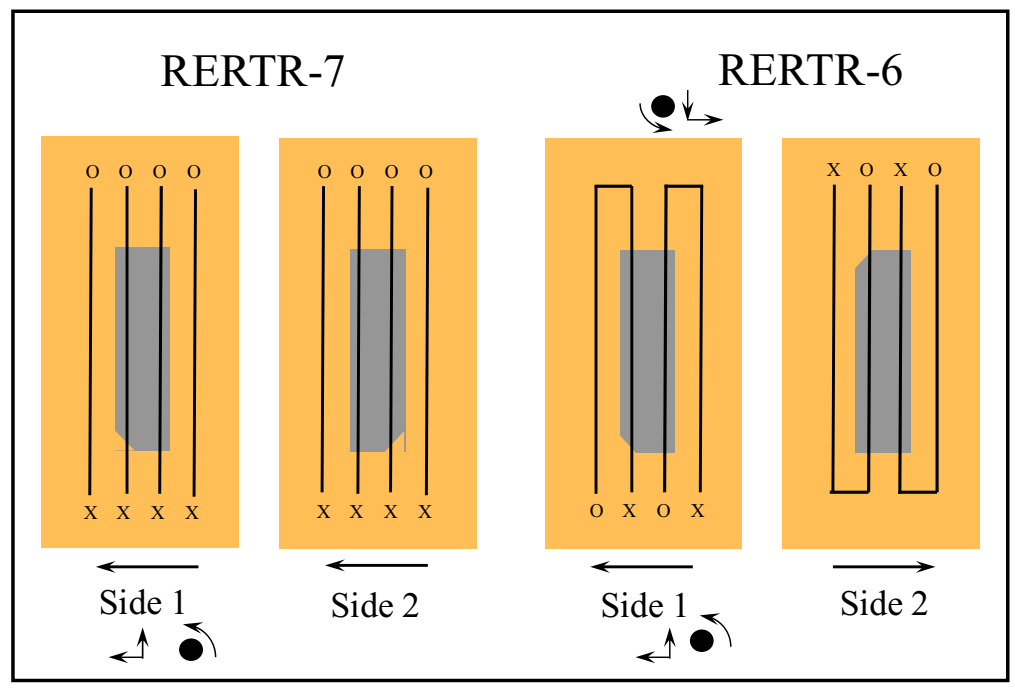

Figure 2.7 Two of the raster patterns used for overlapping FB passes during monolithic fuel fabrication at the INL. The raster pattern used for the RERTR-7 (and subsequent) plates takes more time but causes less plate distortion during the FB process than the raster pattern used for RERTR-6 plates. (Burkes, Keiser and Miller, et al. January 2007a)

FB monolithic fuel was first irradiated in the RERTR-6 experiment, and has been included in every RERTR irradiation experiment since, with various iterations of changes in the FB fabrication equipment, procedure, and fuel materials. The first full-sized monolithic plates planned for irradiation testing, in the AFIP and IRIS-5 experiments, are being fabricated by FB at the INL (Clark, Jue, et al. October 2006). Parametric evaluations (including this thesis study) of temperature and tool effects are ongoing in an effort to understand and improve the FB process and resulting fuel plate quality [(Burkes, Keiser and Wachs, et al. March 2007) and (D. M. Wachs, D. D. Keiser, et al. Sept 2007)]. Thermocouple measurements taken during a 2007 FB test, using a tungsten alloy "Anviloy" tool face, indicated plate interface temperatures of $310-360^{\circ} \mathrm{C}$ under the advancing edge of the tool, $270-360^{\circ} \mathrm{C}$ under the pin, and $110-200^{\circ} \mathrm{C}$ under the retreating edge of the tool. (Burkes, Hallinan and Shropshire, et al. 2008) Figure 2.8 shows the effect of too-high or too-low plate surface temperatures during FB. These correspond with "hot" and "cold" FSW welds discussed previously. 


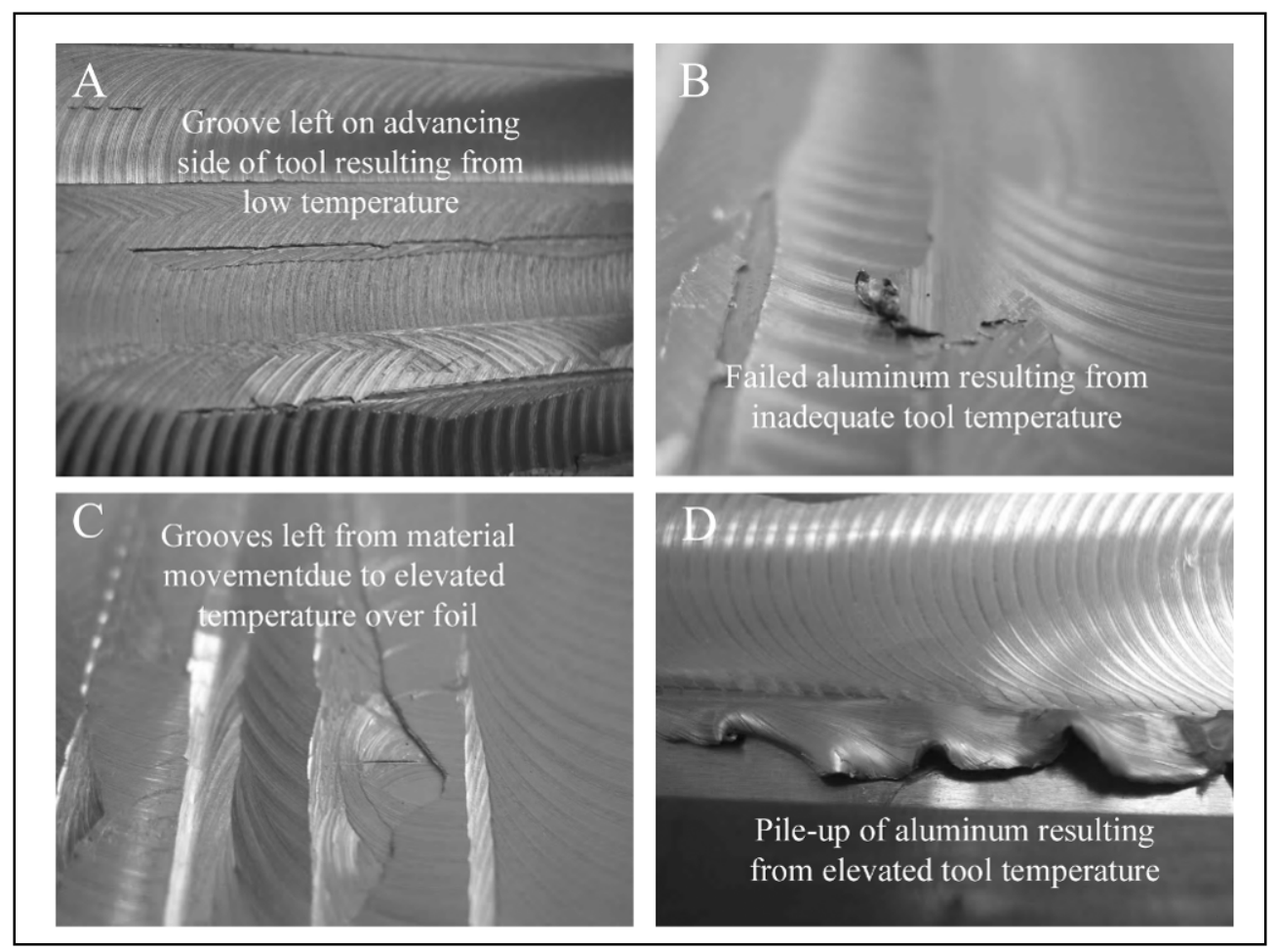

Figure 2.8 Fabrication challenges associated with undesirable FB temperatures. Photographs A and B show impacts of too low a temperature, while photographs $\mathrm{C}$ and $\mathrm{D}$ show impacts of too high a temperature. (Burkes, Hallinan and Shropshire, et al. 2008)

Studies and improvements to the FB process are ongoing:

- Experiments are being conducted with different FB tool face materials. FB processing seems easier with an Anviloy tool material (Clark, Moore, et al. 2007), and samples exhibit good bond strength during pull tests (Burkes, Keiser and Miller, et al. January 2007a). (This thesis study provides further research on FB fuel plates made with various tool materials.)

- (Burkes, Hallinan and Shropshire, et al. 2008) reports tungsten tracer tests conducted in order to investigate lateral material movement during FB. A pocket $45.7 \mathrm{~cm}$ (18 in) long $\mathrm{x}$ $0.635 \mathrm{~cm}(0.25 \mathrm{in})$ wide $\mathrm{x} 50.8 \mu \mathrm{m}$ (0.002 in) deep was milled in the bottom aluminum plate on each of four assemblies. The pocket was filled and leveled with -325 mesh $(<44$ $\mu \mathrm{m}$ diameter) tungsten particles. The intent was for the tungsten particles to simulate a fuel foil, and show movement of the fuel should the monolith foil be disturbed. A single FB pass with one of four target loads was made on each assembly on one side only. Assemblies were then subjected to radiography where materials of different density, i.e. aluminum compared to tungsten, are visible.

Figure 2.9 shows the radiographs of the tungsten tracer test plates fabricated with different FB tool loads. The radiographs show the location of tungsten particles, clearly concentrated around their initial placement. Selected sections of the radiographs have been expanded to clearly show any potential particle movement, shown on the right of the figure. A target applied load of $35.6 \mathrm{kN}(8,000 \mathrm{lbf})$ appears to produce the most particle movement swept towards the tool's advancing side, shown on the lower side of the figure, while the $44.5 \mathrm{kN}(10,000 \mathrm{lbf})$ target applied load produces the least particle movement out 
of the four tests. The $53.4 \mathrm{kN}(12,000 \mathrm{lbf})$ target applied load shows some particle displacement creating a "halo" towards the advancing side of the tool. This effect is enhanced with the $62.3 \mathrm{kN}(14,000 \mathrm{lbf})$ target applied load, in addition to a halo appearing on the tool's retreating side, shown on the upper side of the figure.

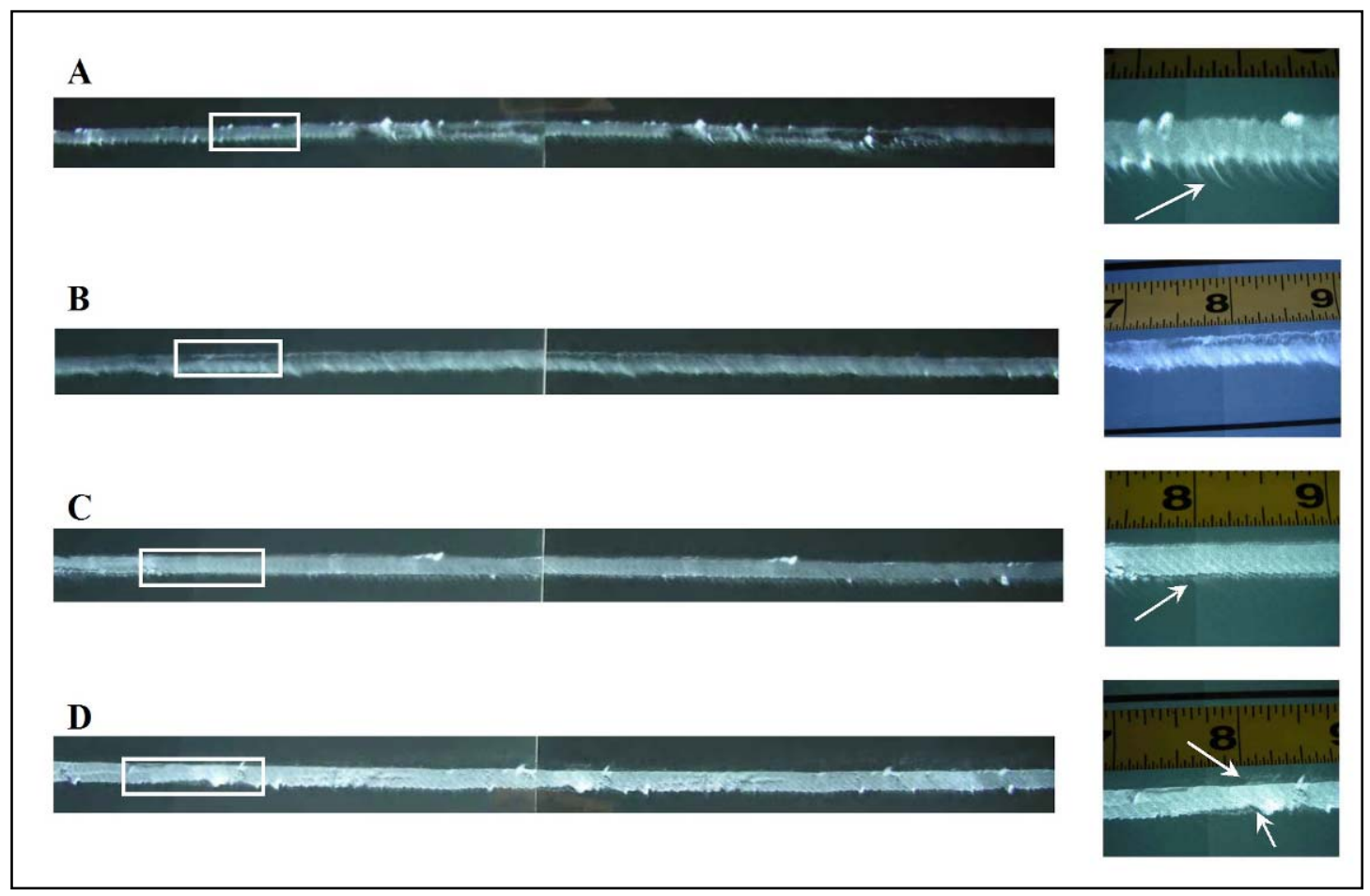

Figure 2.9 Radiographs of FB tungsten particle tracer plates.

Each plate was fabricated with a different FB tool load: A. $35.6 \mathrm{kN}(8,000 \mathrm{lbf})$, B. $44.5 \mathrm{kN}(10,000 \mathrm{lbf})$, C. $53.4 \mathrm{kN}(12,000 \mathrm{lbf})$ and D. $62.3 \mathrm{kN}(14,000 \mathrm{lbf})$. Overall plate radiographs are provided on the left while the insets on the right provide a closer view of the tracer particles. Arrows point to particles that significantly moved outside their original position. The scale for the inset images is in inches $(1 \mathrm{in}=2.54 \mathrm{~cm})$. (Burkes, Hallinan and Shropshire, et al. 2008)

- During efforts by the French to fabricate monolithic fuel plates, FB did not produce acceptable bonding, so they have design efforts underway on a new tool design with changed shoulder and pin diameters. Meantime, they are also investigating a proprietary "new welding technique." (Jarousse, Lemoine, et al. 2006)

- To make FB mini-plate assemblies, a set of multiple foils is typically assembled between two aluminum plates and FB is performed on the entire set. Individual fuel plates can then be cut from the set. A recent change in this FB mini-plate fabrication practice was implemented. Starting with the RERTR-9B fuel plates, the foil arrangement between the aluminum plates was changed to a straight-line arrangement rather than staggered, so that all the foils are clamped down together. Prior to this change, more deflection of foils occurred when they were in the staggered arrangement during FB as illustrated in Figure 2.10. In fact, more such deflection occurred with an Anviloy FB tool face than with one of steel. (Burkes, Hallinan and Wight, et al. September 2007) 


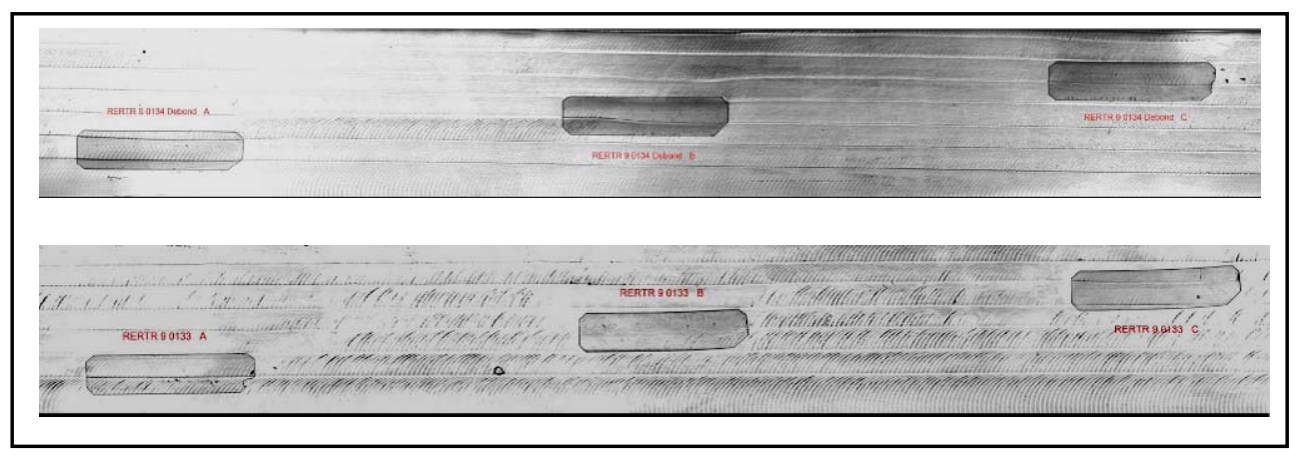

Figure 2.10 UT scans of fuel plate sets fabricated in staggered arrangement using FB tool face of Steel-A36 (top) and Anviloy (bottom). (Burkes, Hallinan and Wight, et al. September 2007)

- A 3-dimensional heat transfer model recently developed at the INL predicts temperature distributions during FB as a function of fabrication parameters such as tool traverse speed, RPM, and load. The model includes the tool, aluminum plate, and steel anvil, but no foil. Advancing and retreating edge effects are simulated by inputting different material properties on each side. The model yields a good match between predicted relative temperatures and actual measurements.

Figure 2.11.A shows photographs of an aluminum plate with one FB pass performed on one side. Observation of the bond shift evident on the plate's bottom side (right photo) indicates that more bonding has occurred on the advancing side, therefore a higher temperature must have existed on that side during the FB process. As the tool rotates, the advancing side is forced in opposition to the new plate material. It carries with it some aluminum material that is already hot, plus it is moving into cold material where viscosity and friction forces are greater and the tool rotation will create more heat. The retreating side also carries hot material with it, but it moves in the same direction as the plate, so its frictional heat is less. The result is an asymmetrical temperature distribution across the bond centerline. The advancing side should therefore exhibit a higher temperature than the retreating side, causing the bond to form farther from the tool center as shown in Figure 2.11.A. Consistent with this interpretation of the bond shift, the model in fact predicts the advancing side of the tool is hotter than either the retreating side or the pin, as is evident from its output illustrated in Figure 2.11.B. (Dixon, Burkes and Medvedev 2007) 


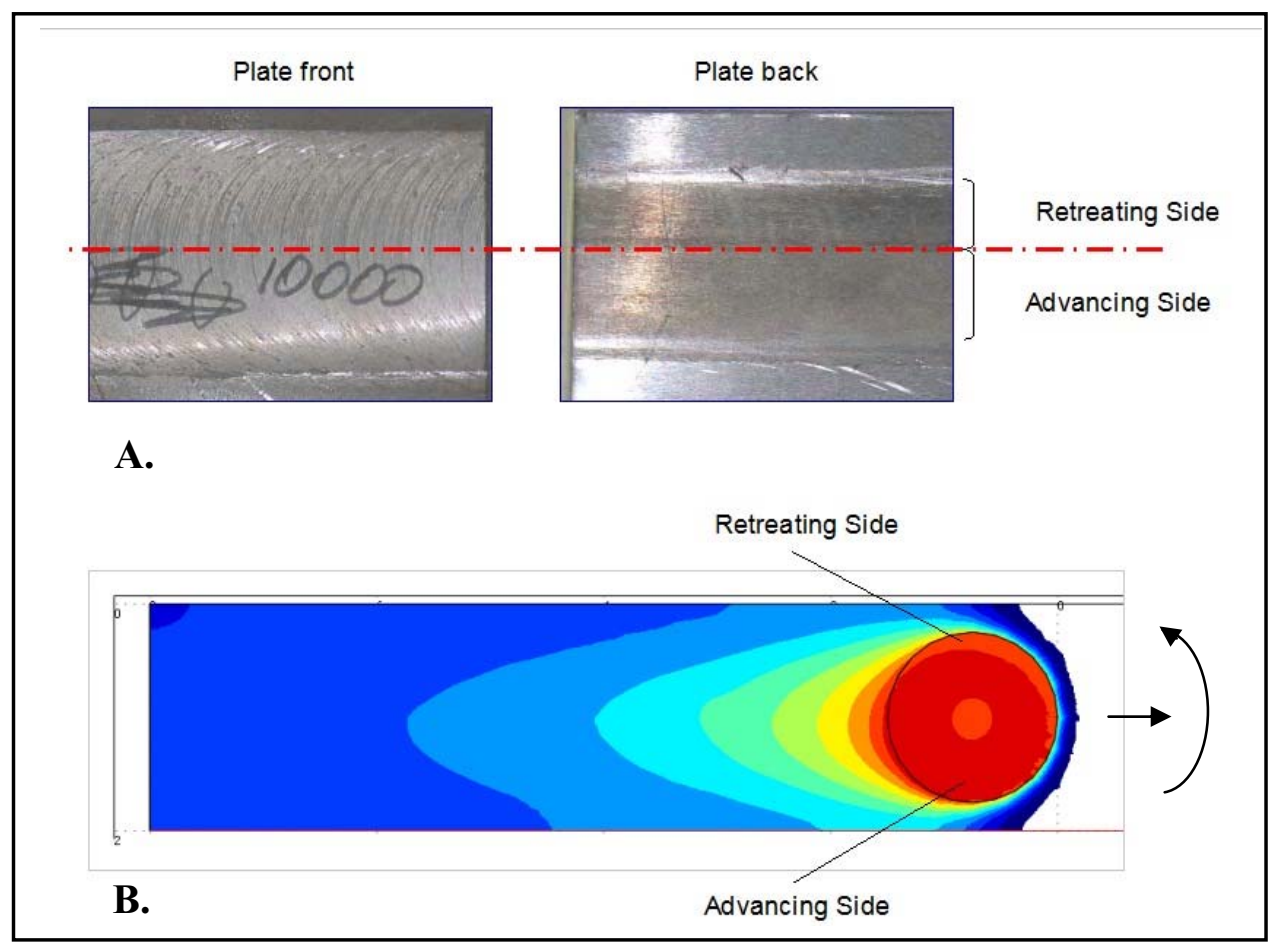

Figure 2.11 The tool's advancing side is hotter than the retreating side.

A. Photographs of a FB plate top side (left) and bottom side (right) showing a shift in the bond line toward the advancing side. B. The heat transfer model developed to simulate the FB process creates a thermal profile of the tool and surrounding material, showing the pin and retreating edge are cooler than the advancing edge, thus confirming the experimental observation. (Dixon, Burkes and Medvedev 2007)

\subsubsection{Monolithic Fuel Examination Findings}

The thesis Introduction section outlines examination methods used at the INL to characterize and evaluate fuel plates. The examinations have several purposes including increased insight into fuel performance in the reactor, and gaining understanding of different fabrication processes or parameters and their impacts on fuel quality. If available, preirradiation data gathered to better comprehend relationships between processing, fuel plate properties, and microstructure are compared with post-irradiation observations to understand radiation effects on the fuel. Ideally, an ensemble of information is collected and analyzed (Burkes, Keiser and Miller, et al. January 2007a). This section discusses findings of some of the monolithic fuel examinations, especially on FB fuel plates. Examination methods can be classified as either irradiation-performance evaluation or pre-irradiation examination methods.

\section{a. Findings of Irradiation-Performance Evaluations of Monolithic Fuel}

- Modeling of irradiation performance - Several models have been developed to help predict fuel plate behavior during irradiation. One such code is PLATE (Plate Lifetime Accurate Thermal Evaluator), which shows success in predicting thermo-chemical performance of dispersion fuels. A version is under development for monolithic fuels, where thermomechanical performance is also an important aspect. So far a flat-plate monolithic fuel 
model has been produced: it applies thermal expansion stresses to evaluate consequent shear forces and strains at the fuel-cladding interface. The flat-plate model of monolithic plates has been used with application of various reactor power densities and operating temperatures, to evaluate shear and normal stresses due to debonds of various sizes, shapes, and gaps. Of the evaluated geometries, it was determined that the worst stress would be $240 \mathrm{MPa}(35,000 \mathrm{psi})$ normal stress occurring on a plate with a $0.100 \mathrm{~cm}^{2}\left(0.155 \mathrm{in}^{2}\right)$ rectangular-shaped debond of $10 \mu \mathrm{m}$ gap, at $45,000 \mathrm{~W} / \mathrm{cm}^{3}$ power density. Future PLATE development will explore hydraulic loads, buckling stresses, arc-shaped plates, and highsilicon fuel materials. The model will be used to help determine maximum acceptable size of debonds and minimum acceptable strength of fuel-cladding interface. [(Wachs, Burkes, et al. Oct 2006b) and(Burkes, Keiser and Miller, et al. January 2007a)]

(Kim, et al. 2007) describes another model which predicts gas bubble swelling in fuel plates during irradiation. Originally developed for dispersion fuels, it has now been revised and updated with data from monolithic fuels, and is now more accurate.

- Post-irradiation examination (PIE) - The first irradiation of monolithic mini-plates was conducted in RERTR-6 at moderate power and burnup levels, with ten plates all fabricated by FB with a steel-faced tool. PIE showed that the monolithic concept was feasible and yielded encouraging results but indicated that the interface between the foil and the cladding was likely to present problems; evidence of incipient bubble formation in the interaction layer and delamination between the foil and the cladding were observed. The delamination correlated with the initial mechanical pull testing results (discussed below under pull-testing), which indicated weak bond strength in a similarly-fabricated surrogate FB plate. [(Finlay, Wachs and Hofman 2006) and (Finlay, Wachs and Robinson, et al. 2007)]

Monolithic fuel has been included in all RERTR reactor experiments since RERTR-6, but PIE data are only available up through RERTR-7, which included monolithic plates fabricated by FB and TLPB at the INL, and by rolling in Zircaloy cladding by CNEA. With availability of PIE data from RERTR-7, it was possible to evaluate the behavior of the monolithic fuel plates at much higher fission densities and burnup levels. [(Finlay, Wachs and Robinson, et al. 2007) and (Robinson and Finlay 2007)]

Certainly one of the most significant and consistent PIE findings for both dispersion and monolithic fuel plates has been the presence of reaction layers at the UMo-Al interface, discussed in more detail in Appendix A. As the amount of burnup increases, so does the amount of fuel swelling, due to more formation of fission gas products and increased reaction layer thickness. Swelling of monolithic fuel relative to burnup appears to be about the same as for dispersion fuel. Occasionally PIE has found gaps due to delamination at the interface; evidence indicated that this delamination occurred after irradiation (such evidence was the presence of a reaction layer, which could not have formed unless the fuel and aluminum were in contact). The delamination was probably due to stress when the sample was cut, but whenever it occurred it indicates a weakened interface bond. PIE also reveals that all the observed irradiation effects - swelling, gas bubbles, reaction layer, and delamination - are mitigated by increased Mo content in the uranium and increased Si in the aluminum as explained in Appendix A. [(Wachs, Ambrosek, et al. Oct 2006a), (Finlay, Wachs and Hofman 2006), (Finlay, Wachs and Robinson, et al. 2007), and (Robinson and Finlay 2007)]

During RERTR-7 irradiation, one of the TLPB plates cracked open and released $50 \%$ of its fuel into the reactor coolant. PIE showed that a similar failure was imminent on another 
TLPB plate. The crack was thought to be caused by Si embrittlement of the Al-Al bond. [(Finlay, Wachs and Robinson, et al. 2007) and (Robinson and Finlay 2007)] No TLPB plates have been irradiated since RERTR-7.

PIE has typically included measurement of plate thickness and metallography of crosssectioned fuel plates. Efforts are underway to expand the current PIE process at the INL to include mechanical pull-testing in future. (Burkes, Keiser and Miller, et al. January 2007a)

\section{b. Findings of Pre-Irradiation Examinations of Monolithic Fuel}

- $\quad$ Ultrasonic testing (UT) - UT is a non-destructive examination method performed on fuel plates submerged in a shallow water tank. The ultrasonic waves pass through materials or are reflected at interfaces of materials of differing densities. Debond-UT scans of fuel plates are examined to determine whether acceptable bonding has occurred between the fuel foil and cladding on all regions. An example of a debond-UT scan of a FB fuel plate set is given in Figure 2.12. Light [white] areas suggest acceptable bonding between the AlAl cladding. Gray areas show acceptable bonding between the foil-cladding interfaces. Dark-colored regions over the gray foils suggest either a debond between the foil and cladding, or an inclusion or impurity in the fuel foil. [(Burkes, Keiser and Wachs, et al. March 2007) and (Clark, Jue, et al. October 2006)] Texture of FB scallops in the aluminum and folds in the bent foils are visible in the UT scans. Dark specks in the white regions represent either debris of thermocouples destroyed in the FB process, or pieces of fuel broken and scattered into the aluminum during the FB process. Note that a UT scan indicates the presence of bond, but does not indicate the strength of the bond. It is possible that a weak bond will break during the process of cutting a sample from the plate.

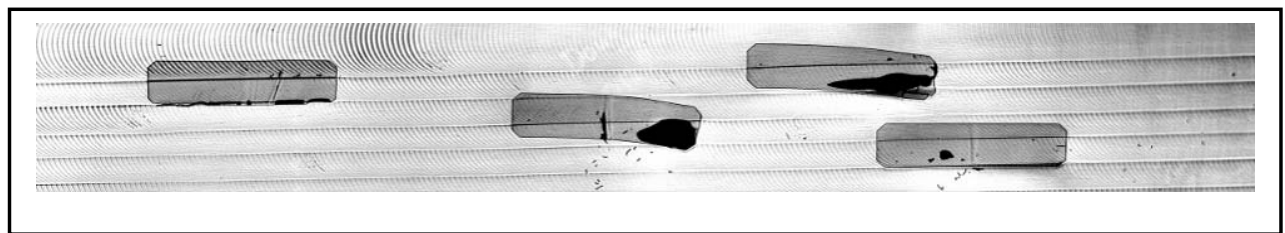

Figure 2.12 Debond-UT scan of FB fuel plate set, fabricated using tool face of Steel-A36.

- $\quad$ Radiography $-X$-ray radiography is another non-destructive examination method. It is performed on every fuel plate to identify the boundaries of the fueled region, to detect fuel outside those boundaries, and to measure fuel density with respect to standard specimens. Figure 2.13 shows an example radiograph of a FB plate set. A radiograph does not indicate bond or debond, but clearly shows locations of fuel foils and other fuel pieces. Fuel particles can be easily distinguished from other debris (e.g., broken thermocouples) by their brighter appearance on the radiograph.

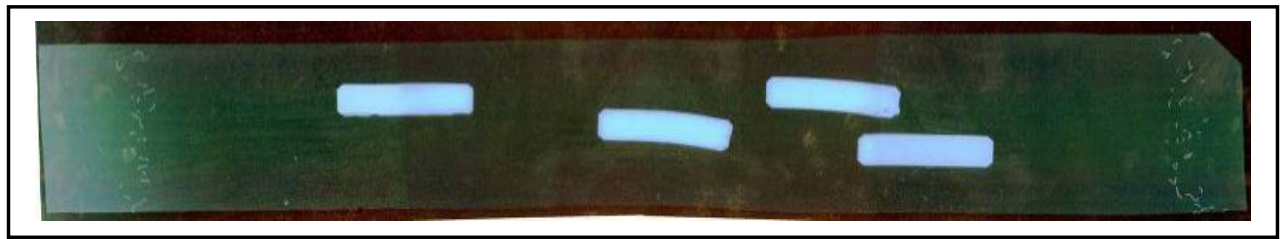

Figure 2.13 X-ray radiograph of FB fuel plate set, fabricated using tool face of Steel-A36. 
- Mechanical testing:

- Bend testing - Figure 2.14 shows steps in performance of a bend test. If any visual delamination is noted, the bond is considered unacceptable (Burkes, Keiser and Miller, et al. January 2007a). Pre-irradiated samples of Al-Al cladding bonds created by all three monolithic bonding fabrication methods have successfully passed the bend testing (Clark, Jue, et al. October 2006). UMo-Al bonds were not bend tested.

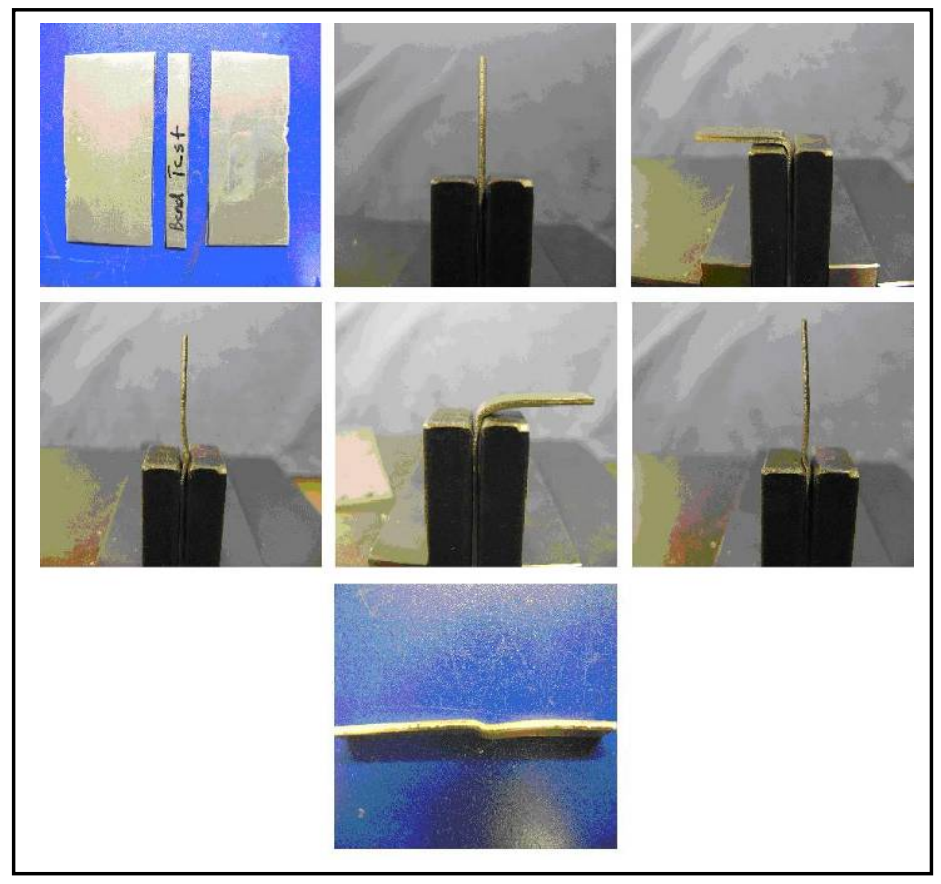

Figure 2.14 Bend testing is performed on a sample of aluminum-aluminum cladding by incrementally bending it one direction over a small radius for $90^{\circ}$, back for $180^{\circ}$, then returning it to its original orientation.(Burkes, Keiser and Miller, et al. January 2007a)

- Pull testing -The fuel-cladding interface is planar, and forces exerted on the interface due to thermal expansion and fuel swelling manifest primarily as stresses normal to the interface plane (Burkes, Keiser and Miller, et al. January 2007a). Therefore, tensile pull tests normal to the interface plane are one important indicator of bond strength. Figure 2.15 shows the test apparatus used to perform pull tests in room temperature at quasiconstant strain rates, on fuel plate samples fabricated by TLPB, HIP, and FB. Each test specimen was a square approx $0.876 \mathrm{~cm}(0.345 \mathrm{in})$ on edge, cut using a low-speed saw, and epoxied onto the test platens. (Burkes, Keiser and Wachs, et al. March 2007) Samples were prepared as described by (Burkes, Keiser and Miller, et al. January 2007a):

- $\mathrm{TLPB}-6.89 \mathrm{MPa}(1,000 \mathrm{psi})$ at $590^{\circ} \mathrm{C}$ for $15 \mathrm{~min}$

- $\mathrm{HIP}-103 \mathrm{MPa}(15,000 \mathrm{psi})$ at $580-\mathrm{C}$ for $90 \mathrm{~min}$

- FB - $406 \mathrm{RPM}, 1.5 \mathrm{~cm} / \mathrm{sec}(37 \mathrm{in} / \mathrm{min})$, - Steel tool face, with load later estimated at roughly $18 \mathrm{kN}(4,000 \mathrm{lbf})$

- Anviloy tool face at $44.5 \mathrm{kN}(10,000 \mathrm{lbf})$ target load

Figure 2.16 shows the pull test results. The samples showing the greatest bond strengths are those that were fabricated by HIP and by FB with the Anviloy tool face; in fact the epoxy failed in these tests prior to failure of the samples' bonds. The FB 
sample made with the steel tool face performed the worst. The difference in the FB samples' pull test results has lead to the conclusion that, for FB samples, there is a dependence of the bond strength at the foil/cladding interface on the tool face material employed in performing the actual FB process. (Keiser, Jue and Burkes 2007)

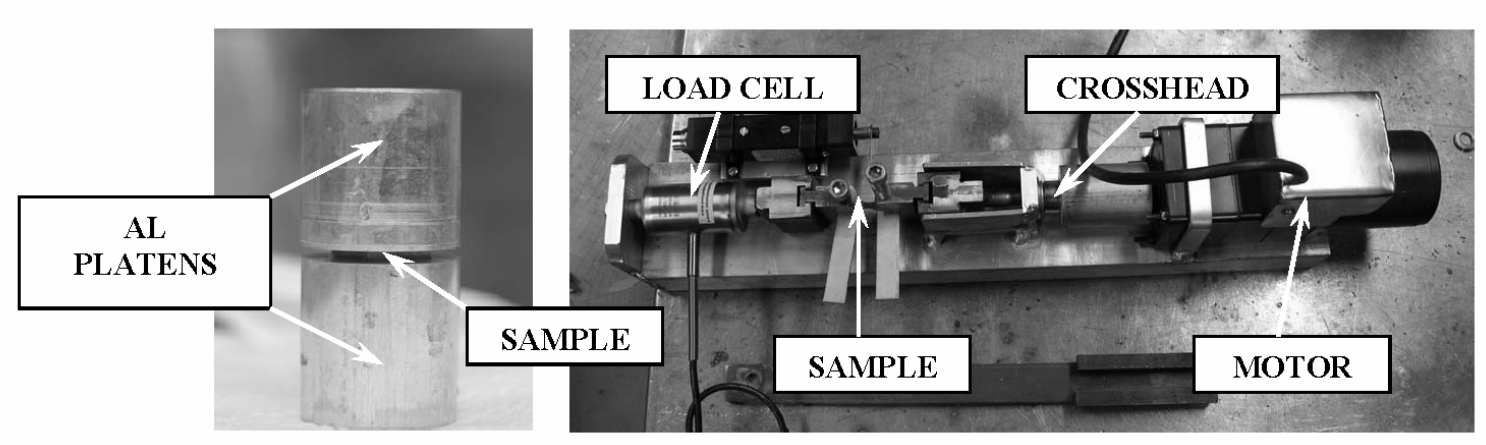

Figure 2.15 Test apparatus used to perform pull tests on fuel plate samples. (Burkes, Keiser and Wachs, et al. March 2007)

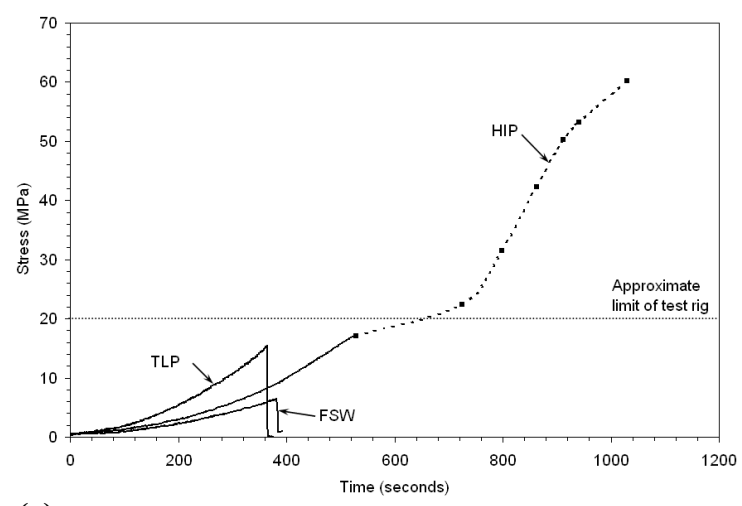

(a)

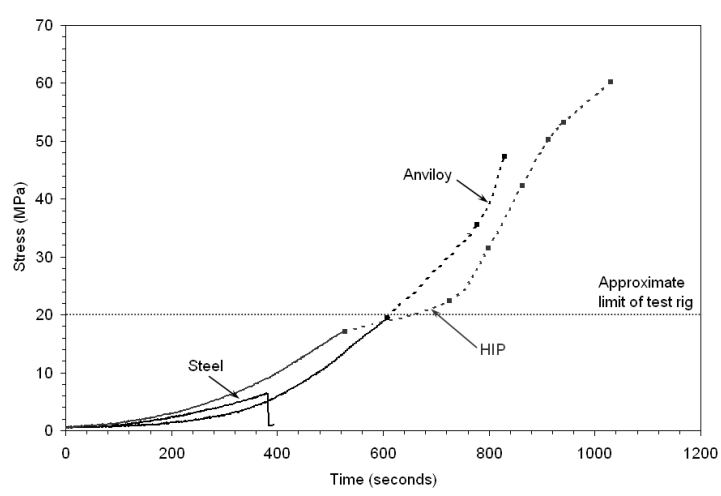

(b)

Figure 2.16 Results of mechanical pull tests on monolithic fuel.

(a) Results of initial pull test series with samples that were prepared by TLPB, HIP, and FB with a steel tool; (b) Results of a later pull test on a sample prepared by FB with an Anviloy tool, shown together with some previous results for comparison. [(Burkes, Hallinan and Wight, et al. September 2007) and (Burkes, Keiser and Miller, et al. January 2007a)]

- Microstructural examination - Metallography of cross-sectioned surrogate fuel plates inspect many of the same parameters as during the PIE microstructure examination: interface reaction layer development, material swelling, grain size, hardness testing, and bond delamination (Keiser, Jue and Burkes 2007). Samples cut from the fuel plates are mounted and etched to exhibit the fuel cross-sections and their microstructure, examined via electron or optical microscopy, and sometimes microhardness tested.

Such examination observed that the FB fuel aluminum cladding exhibits homogeneously fine grain structure with no sign of recrystallization structure, and interpreted this as typical of FSW TMAZ regions. Meantime, the UMo foil retains its classic as-rolled elongated grain structure even after the FB process. [(Keiser, Jue and Clark 2006) and (Clark, Hallinan, et al. May 2006)] 
Description of chemical reaction layers at the UMo-Al interface observed during metallography of monolithic fuel samples fabricated with TLPB, HIP and FB are available in Appendix A.

Bond evaluation during microstructural examination includes estimating the percent bonding by visual examination of grain growth across a bond line throughout a sample, viewed at 50X magnification. A rule-of-thumb for adequate bonding is considered to be $50 \%$ percent minimum.

The FB uranium-aluminum bonds are thought to be physical (intermolecular) rather than chemical (intramolecular). However, the RERTR Program has not yet investigated the nature of the bonding mechanism. ${ }^{11}$

The development of fabrication processes that yield high strength bonds, and of fuel systems that resist the degradation of those bonds, are of primary importance. Bonds are nondestructively examined by UT, and destructively examined by cutting samples from fuel plates and evaluating them in a number of ways. The UT, radiography, mechanical testing, and microstructural exams, together with PIE data, create an ensemble of information about the fuel plates. Of primary interest are those data relating to bond strength and integrity. [(Clark and Briggs 2004), (Burkes, Keiser and Wachs, et al. March 2007), and (D. M. Wachs, D. D. Keiser, et al. Sept 2007)]

\footnotetext{
${ }^{11}$ Investigation into the nature of FB's molecular bonding is outside the scope of this thesis research.
} 


\subsection{THESIS RESEARCH OBJECTIVE}

The objective of this thesis research is to provide insight into the FB process and its application to the monolithic fuel design by recognizing and understanding the microstructural effects of varying fabrication parameters (a) FB tool load, and (b) FB tool face alloy.

As previously discussed, monolithic nuclear fuel is still under development, as is the FB process. Neither FB nor FSW have been used in any fuel fabrication or nuclear application before the RERTR program. Research is needed because there is an incomplete understanding of the impact that some changes in FB fabrication parameters have on monolithic fuel plate characteristics. A previously unexplored and needed contribution to such research is provided by this thesis study's microstructural examination of materials and bonds in unirradiated FB plates fabricated using varied FB tool loads and tool face materials. It is expected that these parameters help drive material movement and temperature during fabrication, and thus the material properties, thermal expansion and residual stresses, and formation of interface reaction layers. The RERTR program is just starting to understand these drivers and their effects - this study contributes to that understanding by recognizing symptoms of differences in the microstructure that result when the plates are FB fabricated with variation in these parameters.

Innumerable steps are required to develop and validate a new design for nuclear fuel. This research is significant because it provides some of those steps; it promotes understanding of the impacts of varying FB tool loads and tool face materials, and thus gives insight to the applicability of the FB process to fabrication of monolithic fuel. Based on this research, a possible range of FB tool loads, and a specific FB tool face material can be selected and applied for future monolithic fuel fabrication. Examination results are now available to researchers for comparison with existing or future data on similar samples. Such samples will come from irradiated fuel where PIE data are available, or will represent fuel made with other FB machines, or with known differences in other FB fabrication parameters. These research results and future comparisons will ultimately help determine whether and how FB can be used to fabricate fuel plates, specifically the acceptable range of FB tool load and the preferred FB tool face material. If the RERTR program recommends reactor use of FB monolithic fuel, these examination results will contribute toward validation, qualification, and NRC licensing of that fuel design. 


\subsection{MATERIALS AND METHODS}

\subsection{General Plate Fabrication and Sample Preparation}

Microstructural examinations reported in this study were performed on samples taken from cross-sectioned unirradiated FB plates. The plates were created to represent varied FB fabrication parameters: (a) tool loads, and (b) tool face materials. Table 4.1 provides a list of the samples.

Table 4.1 Samples taken from plates created to represent varied FB fabrication parameters. (a) Two sheets of aluminum cladding friction bonded together using varying FB tool loads; and (b) DU10Mo fuel foil encased in aluminum cladding, all friction bonded together with varying FB tool face materials.

\begin{tabular}{|c|c|c|c|}
\hline \multicolumn{2}{|c|}{ Sample Group } & \multicolumn{2}{|c|}{ Operational Parameter } \\
\hline Material & Sample ID & $\begin{array}{c}\text { (a) } \\
\text { Target FB Tool Load } \\
\text { N (lbf) }\end{array}$ & $\begin{array}{c}\text { (b) } \\
\text { FB Tool Face } \\
\text { Material }\end{array}$ \\
\hline \multirow{4}{*}{$\begin{array}{l}\text { (a) Aluminum }{ }^{\text {a }} \text { cladding } \\
\text { (no fuel foil) }\end{array}$} & $8 \mathrm{~K}$ & $35,600(8,000)$ & \multirow{4}{*}{ Anviloy } \\
\hline & $10 \mathrm{~K}$ & $44,500(10,000)$ & \\
\hline & $12 \mathrm{~K}$ & $53,400(12,000)$ & \\
\hline & $14 \mathrm{~K}$ & $62,300(14,000)$ & \\
\hline \multirow{3}{*}{$\begin{array}{l}\text { (b) } \mathrm{DU} 10 \mathrm{Mo}^{\mathrm{b}} \text { fuel foil } \\
\text { encased in aluminum } \\
\text { cladding }\end{array}$} & Steel & \multirow{3}{*}{$48,900(11,000)$} & Steel-A36 \\
\hline & Al-Bronze & & Al-Bronze \\
\hline & Anviloy & & Anviloy \\
\hline
\end{tabular}

Plates were fabricated and samples were selected, prepared, and examined as described by the following general steps. Details specific to each sample group are described in subsequent sections.

- $\quad$ FB plate fabrication - All the plates were FB fabricated by the same operator using the Kearney \& Trekker milling machine in the FASB facility at MFC. The tool RPM and traverse speeds were automatically held constant by the milling machine, and the tool load was manually adjusted throughout the fabrication to maintain the target load as nearly as possible. The tool load was continuously displayed on-screen, and typically varied during any one pass. The operator manually adjusted the tool load not only to meet the target, but also in response to the visual appearance of the aluminum plate's surface quality during FB. For example, if he observed more material extrusion than should occur, he would reduce the load regardless of whether it went below the target. When the material surface had smoothed back out, he would promptly increase the load. Because there is an inherent time lag between adjusting the load and the hysteresis effects, manual load control is somewhat an art. Therefore even though a target was identified for the FB tool load, the actual load sometimes varied considerably: the variation was typically within less than $\pm 10 \%$ for the aluminum sample group and within roughly $\pm 20 \%$ for the DU10Mo sample group. 
The tool load was measured concurrent with the FB process on all the plates, as was temperature inside the tool by a thermocouple immersed in ethylene-glycol coolant. Data were collected via a $66.7 \mathrm{kN}$ load cell (HiTec Corporation) and Type K thermocouples (Omega Engineering) transmitting ${ }^{12}$ to a PC operating with Labview Version 7.1 software. The tool load and internal temperature, and the coolant inlet and outlet temperatures, were displayed and recorded during FB fabrication of all the plates. No data were measured or recorded of actual plate temperatures.

- $\quad$ Sample cutting - Locations for cross-sectioned samples were selected and marked on the plates perpendicular to the direction of the FB passes. Samples were destructively cut by shearing, with further cuts made with a high-speed saw if needed to remove edges cleanly.

- Mounting - The samples were mounted per typical metallography practices: with the surface of interest facing downward, each sample was set in a clip to hold the sample upright, and placed inside a phenolic ring of 2.5- or 5.0-cm (1- or 2-inch) diameter. Epoxy was mixed on the spot, poured immediately inside the ring, surrounding and embedding the sample and clip, and set up transparently within a few minutes with no further treatment needed.

- $\quad$ Polishing - Each sample's surface of interest was polished to 1200 grit. This surface finish is necessary both for microhardness test procedure requirements and for adequate visual discernment between microstructures and surface roughness.

- Hardness Testing - Vickers Hardness $\left(\mathrm{H}_{\mathrm{V}}\right)$ measurements were taken at multiple locations on each sample according to standard microhardness measurement procedure [ (ASTM 2006b), (LECO 2002), and (Olympus n.d.)]. Standard deviations were calculated and are included in Appendix E.

- Etching - Each sample's surface of interest was etched in acid solution to make the microstructure more clearly visible. The presence of the fuel foil determined the acid solution composition and etching procedure, which therefore differed for the two sample groups.

- Microscopy - Microstructures of the cross-sectioned samples were examined on an optical microscope, and microphotographs were taken (Olympus n.d.). Sample characteristics expected to be helpful in evaluating the FB process and resulting fuel plates included:

- Sample width and thicknesses, and thickness of component layers

- Bonding or delamination between material layers - percent bonding was estimated by visual examination of grain growth or apparent adhesion across the bond line throughout each sample, viewed at 50X magnification

- Bond phenomena, for example, presence of chemical interaction layers (however, investigation of the nature of FB's molecular bonding cannot be performed using optical microscopy, and is outside the scope of this thesis research)

- Material homogeneity, grain size, and shape

- Material hardness

- Evidence of thermal effects on the materials (might be manifested by changes in any of the above characteristics).

\footnotetext{
${ }^{12}$ For the aluminum sample group, the data were transmitted through an Hp Agilent 34970A data logger. For the DU10Mo sample group, data were transmitted through a NIDAC data acquisition unit.
} 
The author of this thesis report was not present during FB fabrication of these plates, although she was present at multiple occasions of FB fabrication of other plates. She also did not select or cut the aluminum samples representing the varied FB tool loads. She did, however, perform all the other sample activities including selection, marking, cutting, mounting, polishing, etching, hardness testing, microscopic examination, and evaluation of findings.

Plate fabrication and sample cutting described above were performed at the MFC FASB facility. Sample polishing, etching, hardness testing, and microscopy were done in the MFC Electron Microscopy Laboratory (EML). Epoxy-mounting of the DU10Mo samples was accomplished at FASB, while the aluminum samples were epoxy-mounted at EML. Equipment photographs are available in Appendix B.

Cutting, polishing, and etching of all samples were performed by trained personnel (including the author) wearing personal protective equipment (PPE) such as lab coats, safety glasses, and gloves. The DU-containing fuel plates and their samples were stored, inventoried, transported, and handled in accordance with INL nuclear material control practices, and preparation of these samples had to be performed in radioactive hoods. Training, qualification, and work approvals constituted a significant part of the effort in performing this work at the INL, with the goal of a resulting safe work environment of highly trained and knowledgeable personnel. Meeting these INL mandates required additional time and care for the thesis research work.

Details specific to sample selection, preparation, and examination for each sample group are described in the following sections.

\subsection{Friction Bonding, Preparation and Examination of Aluminum Samples Fabricated with Varying Tool Loads}

Sample plates of aluminum only were chosen for testing the first parameter since the absence of the DU foil simplified administrative requirements for performing the work. One pass was performed on only one side of the plate assembly so that the FB process could be more easily understood by clearer differences between both sample edge effects and sample sides.

The four aluminum FB samples were prepared and examined during a five-month period. Each sample was taken from a plate fabricated by joining two layers of aluminum (Al-6061T6) plate together with a single FB pass on one side, as illustrated in Figure 4.1. All plates were fabricated as described above, using an Anviloy-faced FB tool. A different FB tool load was targeted during FB of each plate, with monikers " $8 \mathrm{~K}$," " $10 \mathrm{~K}$," " $12 \mathrm{~K}$," and " $14 \mathrm{~K}$ " subsequently used to identify the samples as noted in Table 4.1. Temperature data measured during FB were not saved, but the tool internal temperature was approximately the same $\left(140^{\circ} \mathrm{C}\right)$ during all the FB passes. See Figure 4.2 for photos of tops and bottoms of aluminum plates bonded with FB passes made at the four different tool loads. 


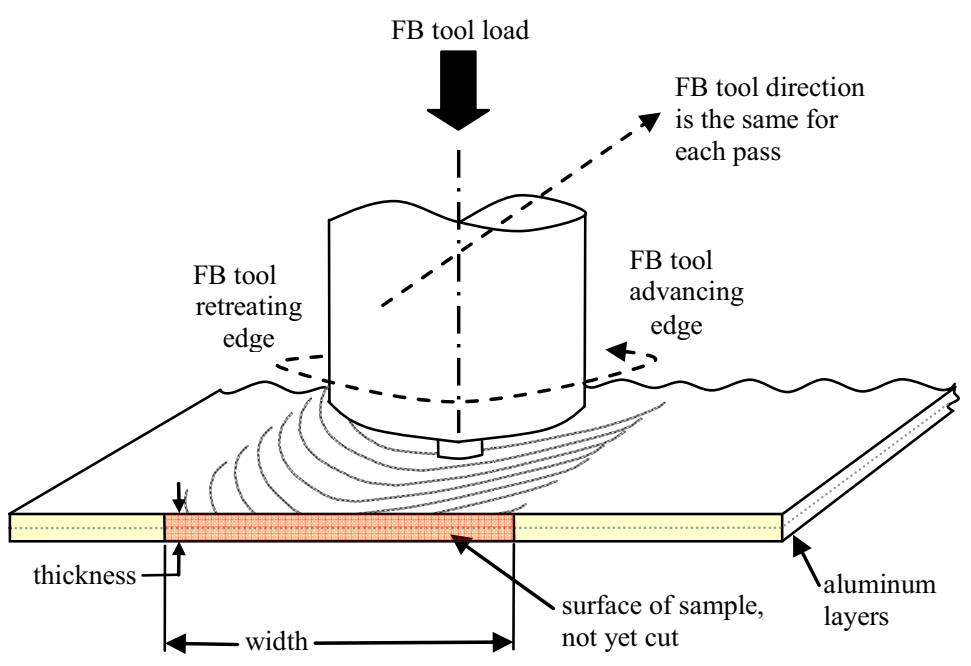

Figure 4.1 One-sided, single-pass FB process, showing direction of FB tool movement and rotation, with resulting orientation of advancing and retreating edges.

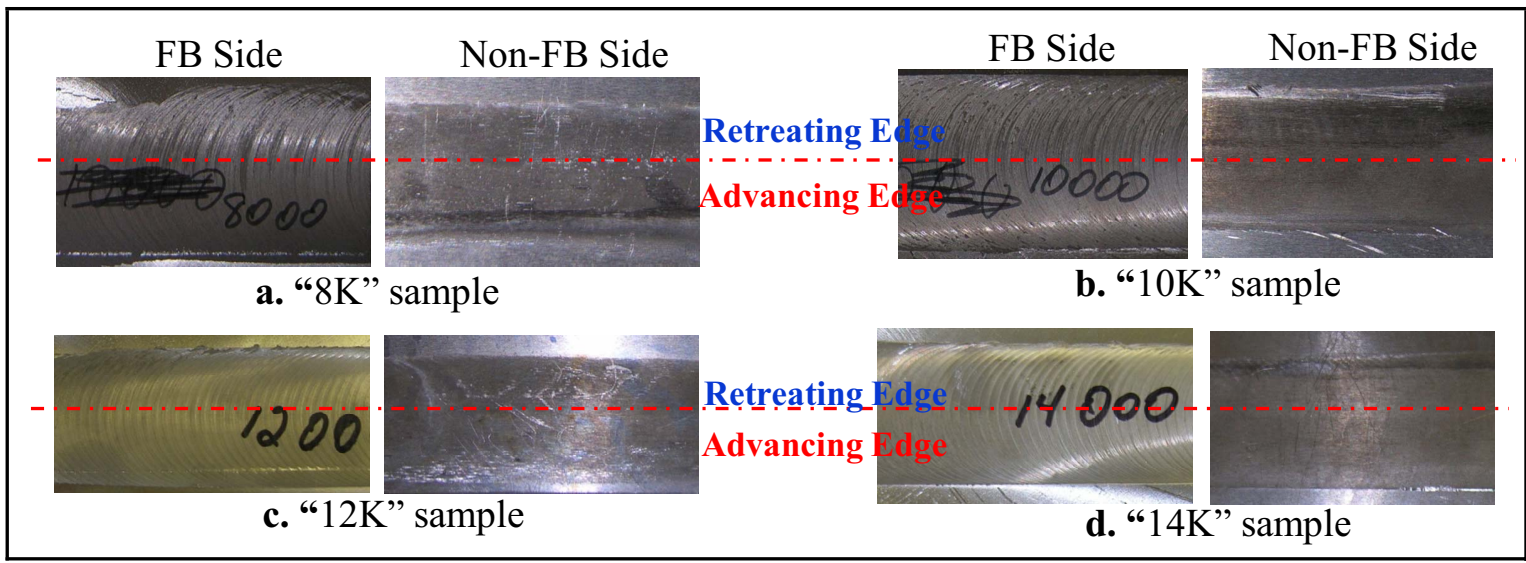

Figure 4.2 Aluminum plates bonded with one-sided, single pass FB, with varying tool load targets of: a. $35,600 \mathrm{~N}(8,000 \mathrm{lbf})$, b. $44,500 \mathrm{~N}(10,000 \mathrm{lbf})$, c. $53,400 \mathrm{~N}(12,000 \mathrm{lbf})$, d. $62,300 \mathrm{~N}$ (14,000 lbf). [after (Burkes, Hallinan and Chapple, et al. January 2008)]. 
After being cut from the plates, the samples were met-mounted as shown in the photo in Figure 4.3. The samples' FB retreating edges were located at the clip end.

The samples were then polished and hardness tested in three or four locations across the sample width on each sample as shown in Figure 4.4. The first hardness test series on each sample was measured at locations approximately in the center of the sample. Since the first location "A" on the $10 \mathrm{~K}$ sample was not in the center, another set of measurements was taken closer to the sample's center. Subsequent measurements were taken on all samples at locations near the retreating edge, advancing edge, and center. At all locations except one, eight hardness measurements were taken across the sample thickness. At the "center" location on the $10 \mathrm{~K}$ sample, only seven hardness measurements were taken.

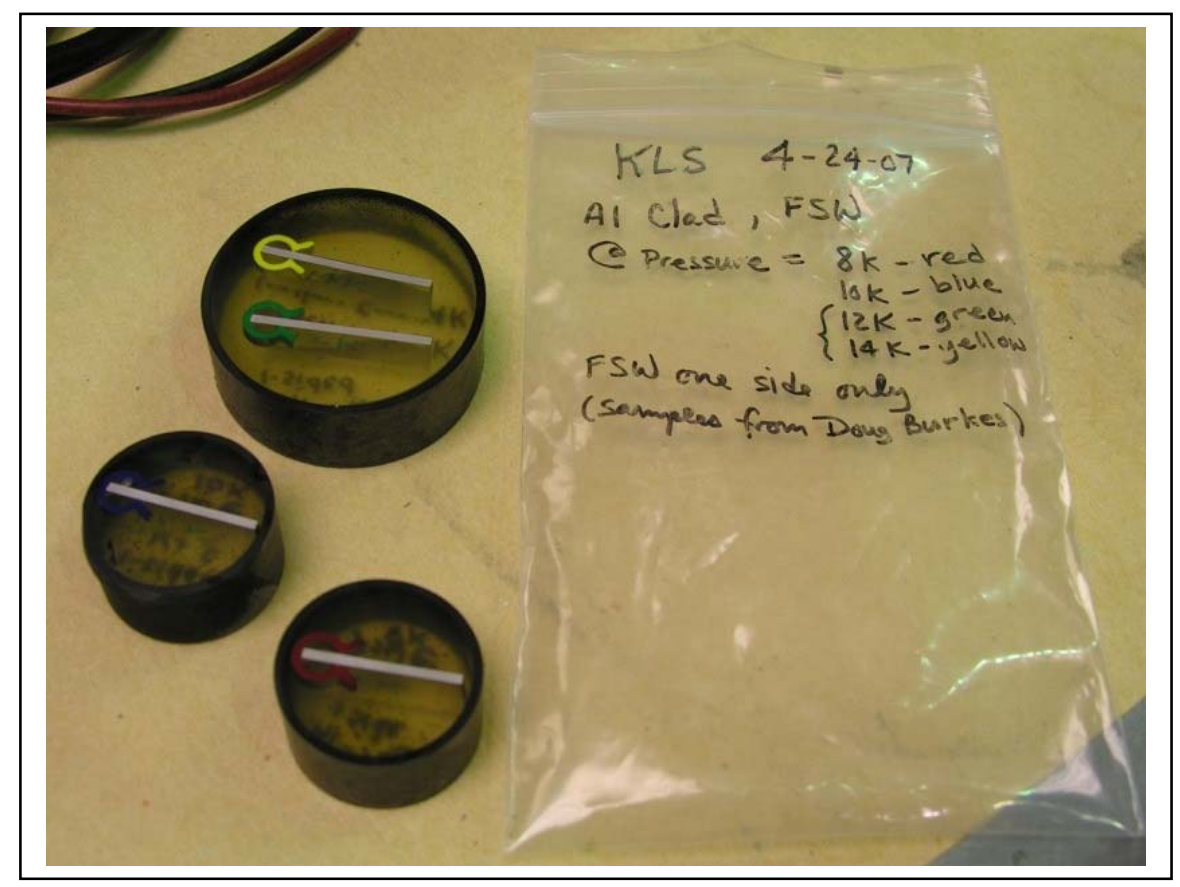

Figure 4.3 Mounted cross-sectioned samples of one-sided, single pass FB aluminum plates, bonded with varying FB tool loads. 


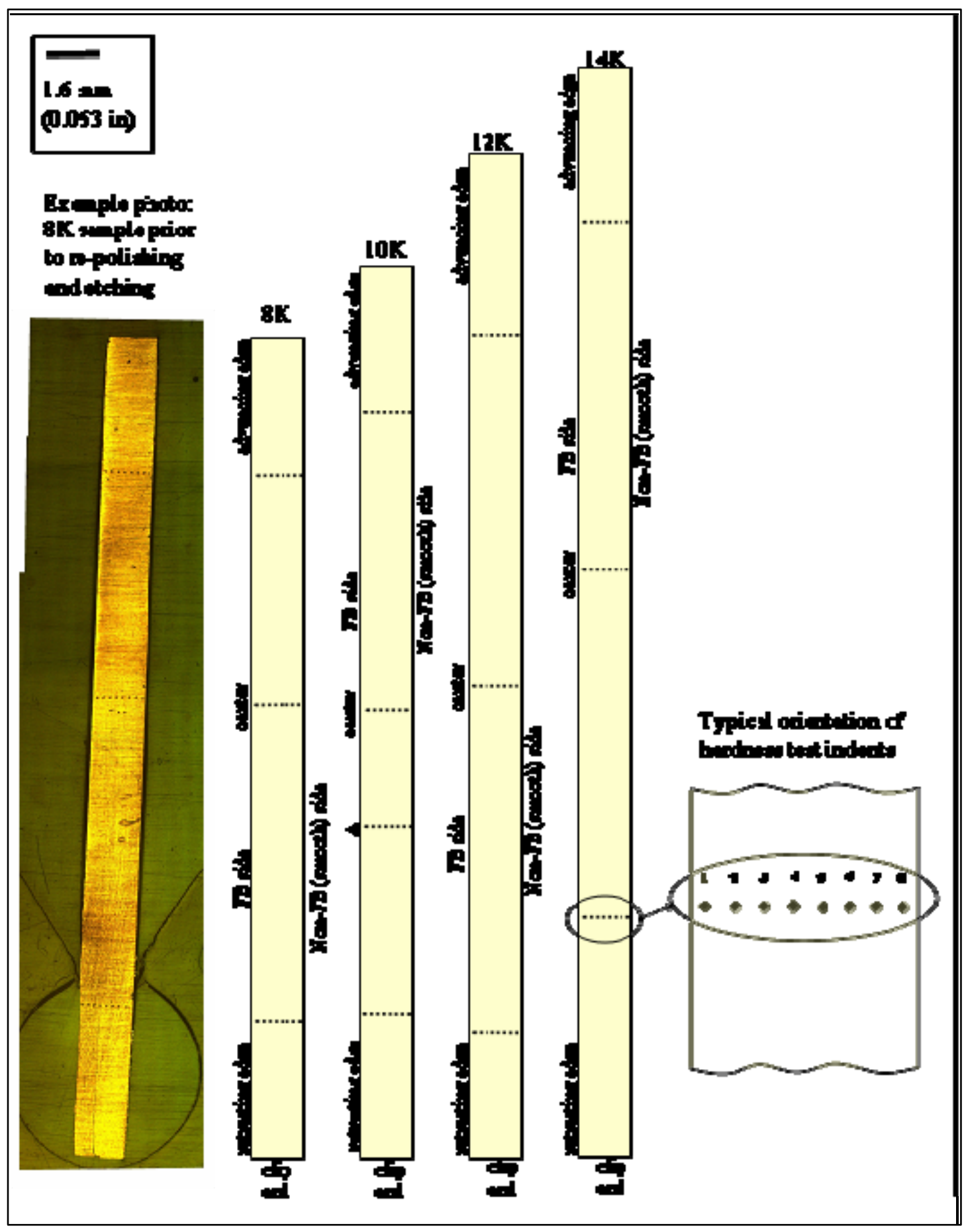

Figure 4.4 Location of Vickers Hardness tests on aluminum samples (to scale) taken from plates fabricated with one-sided, single-pass FB.

Because a few weeks had passed and considerable handling had occurred since they were first polished, the samples were re-polished to assure clean surfaces prior to etching. Hardness was retested in a few locations on the $8 \mathrm{~K}$ and $14 \mathrm{~K}$ samples to confirm hardness consistent with previous measurements. All samples were then etched in a chemical fume hood, with reagent comprised of $190 \mathrm{ml}$ de-ionized water, $5 \mathrm{ml}$ nitric acid, $3 \mathrm{ml}$ hydrochloric acid, and $2 \mathrm{ml}$ hydrofluoric acid. Samples were examined by visual microscopy and microphotographs were 
taken. Grain shape and direction were subjectively and qualitatively determined by visual inspection. Percent bonding was estimated. Grain size was calculated at the center location and clip end location of each sample; these locations were selected because they visually appeared to represent the coarsest and finest grains in the samples, and because they corresponded to locations of some hardness measurements. Average area per grain and the ASTM grain size number "G" were measured on each sample based on the average of two or three standard-area circles in each location, using a planimetric procedure per (ASTM 2004). Note that this method is recommended for equiaxed grain shapes, which these grains did not generally exhibit. Because percent-bonding and grain shape and direction have high variability within each sample, these estimates were based largely on subjective interpretation.

\subsection{Friction Bonding, Preparation, and Examination of DU10Mo Samples Fabricated with Varying Tool Face Alloys}

Fabrication of surrogate foils used in this study was performed per the improved practice described previously. The fuel plate FB was performed as illustrated in Figure 4.5. Two sets of data were gathered and evaluated on FB of DU10Mo fuel plates with varying tool face alloys. The first data set and its evaluation were related to loads and temperatures measured during the general FB process performed on the three entire fuel plate sets. The second was related to three samples, each one taken from a specific location within a fuel plate from each fuel plate set.

\subsubsection{General Friction Bonding Process on DU10Mo Fuel Plate Sets}

Three sets of fuel plates were FB fabricated as generally described in Section 4.1, with FB tool load manually adjusted throughout all their fabrication to maintain a target $48,900 \mathrm{~N}$ $(11,000 \mathrm{lbf})$ load. Each set was fabricated with a different FB tool face alloy: either Steel-A36, an aluminum-bronze alloy, or a tungsten alloy "Anviloy." Some material properties of these alloys are listed in Table 4.2. The plates made with Steel-A36 and Anviloy FB tool faces were archives, representing plates used in the RERTR-9A experiment. All plate sets were comprised of three or four DU10 Mo ${ }^{13}$ foils encapsulated between two layers of aluminum (A1-6061-T6) cladding. Foil and plate fabrication information can be found in Table 4.3. After the FB operations, edges of the bonded plate sets were sheared, and the plate sets were stored. They were later sent for "debond-UT" ultrasonic scans and for radiography. Photos, ultrasonic scans, and radiographs for the three plate sets are provided in Figure 4.6.a, b, and c.

\footnotetext{
${ }^{13}$ DU10Mo is Depleted Uranium alloyed with $10 \mathrm{wt} \%$ Molybdenum. These surrogate fuels were made with DU10Mo foils to represent the RERTR-9A fuel, which had foils of enriched uranium alloyed with $10 \mathrm{wt} \%$ molybdenum to stabilize the uranium $\gamma$-phase, thus reducing U-Al interaction and minimizing fuel swelling, as explained in Appendix A.
} 


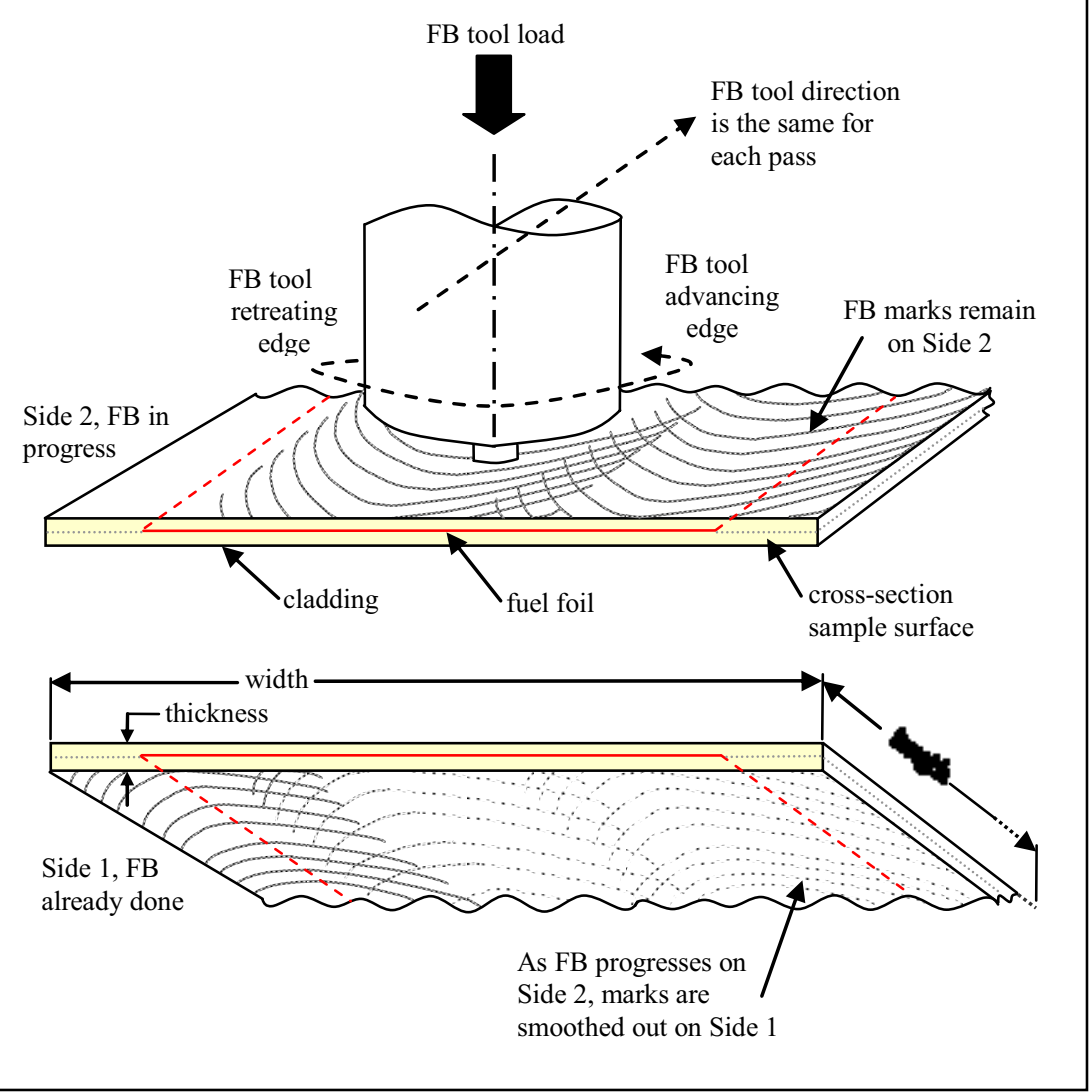

Figure 4.5 Cutaway view of Side 1 (lower figure) and Side 2 (upper figure) of a surrogate fuel plate, fabricated with a multiple-pass FB process to bond DU10Mo foil inside aluminum Al-6061 cladding. FB marks are illustrated on both Side 1 and Side 2, and the cut foil is exposed at the cross-sectioned sample surface.

Table 4.2 Material properties of FB tool face alloys.

\begin{tabular}{|c|c|c|c|c|c|c|}
\hline $\begin{array}{c}\text { FB Tool } \\
\text { Face Alloy }\end{array}$ & $\begin{array}{c}\text { Thermal } \\
\text { Conductivity } \\
\left(\mathbf{W} / \mathbf{m}^{\mathbf{0}} \mathbf{K}\right)^{\mathbf{a}}\end{array}$ & $\begin{array}{c}\text { Specific Heat } \\
\text { Capacity } \\
\left(\mathbf{J}^{\prime} \mathbf{g}^{\mathbf{0}} \mathbf{K}\right)^{\mathrm{a}}\end{array}$ & $\begin{array}{l}\text { Density } \\
\left(\mathrm{g} / \mathrm{cm}^{3}\right)^{\mathrm{a}}\end{array}$ & $\begin{array}{c}\text { Hardness } \\
\left(\text { HRA }^{\mathbf{b}} / \mathbf{H}_{\mathbf{V}}{ }^{c}\right)\end{array}$ & $\begin{array}{l}\text { Machinability } \\
\text { (qualitative) }\end{array}$ & $\begin{array}{c}\text { Wear } \\
\text { Resistance }_{\text {(qualitative) }^{\mathrm{d}}}\end{array}$ \\
\hline Steel-A36 & 47 & 0.418 & $7,850,000$ & $60 / 234$ & 7 & 6 (Mild) \\
\hline $\begin{array}{l}\text { Aluminum } \\
\text {-Bronze }\end{array}$ & 56 & 0.375 & $8,170,000$ & 65 / 297 & 8 & 4 (Poor) $^{\mathrm{e}}$ \\
\hline Anviloy & 167 & 0.142 & $19,376,000$ & $67 / 330$ & 9 & 9 (Very Good) \\
\hline $\begin{array}{ll}\text { a. } & \text { Thermal } \\
& \text { values fr } \\
\text { b. } & \mathrm{HRA}=\mathrm{R} \\
\text { c. } \mathrm{H}_{\mathrm{V}}=\mathrm{Vic} \\
\text { d. } \text { Machinal } \\
\text { being the } \\
\text { e. } \text { The Al-B }\end{array}$ & $\begin{array}{l}\text { Rockwell A-Sca } \\
\text { cker's hardness } \\
\text { bility and wear } \\
\text { e best. }\end{array}$ & $\begin{array}{l}\text { hardness measu } \\
\text { heasurement, def } \\
\text { esistance are rate }\end{array}$ & $\begin{array}{l}\text { nent, defin } \\
\text { d in (ASTN } \\
\text { ased on a s }\end{array}$ & $\begin{array}{l}\text { alues are for the } \\
\text { ase 2007). } \\
\text { in (ASTM 2006 } \\
2006 \mathrm{~b} \text { ). } \\
\text { jective } 1-10 \mathrm{scal} \\
\text { ork hardening, ga }\end{array}$ & $\begin{array}{l}\text { b). } \\
\text { e by the FB ope } \\
\text { alling, and thern }\end{array}$ & $\begin{array}{l}\text { temperature, } \\
\text { tor, with } 10 \\
1 \text { stress. }\end{array}$ \\
\hline
\end{tabular}


Table 4.3 Fabrication parameters for surrogate fuel foil and FB plates from which samples were taken for this study's examinations.

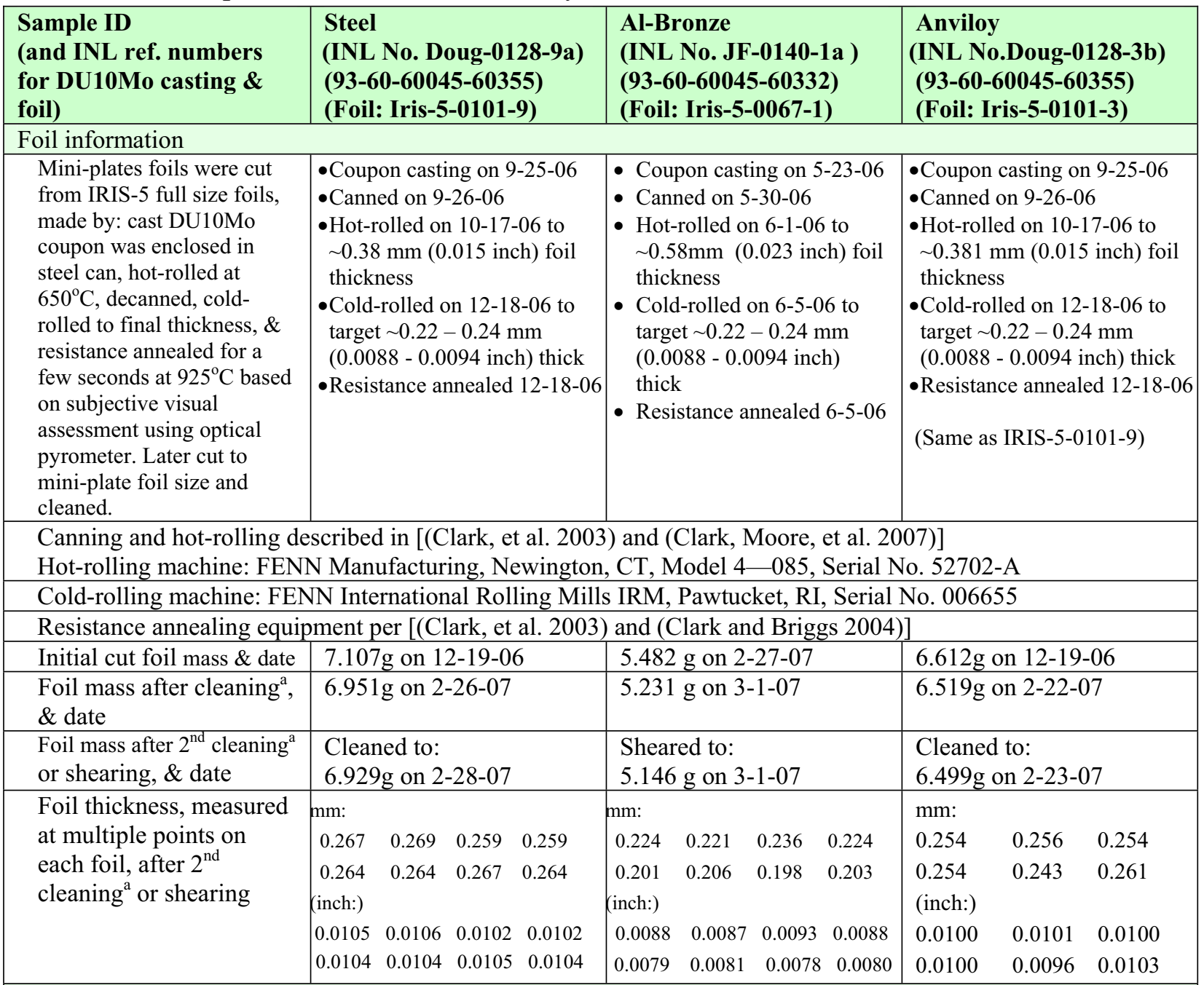

Aluminum plate (Al-6061-T6) cladding information (nominal for typical plates, not specifically measured on these plates)

Nominal thickness of Side $1 \mathrm{Al}$ plate $=0.6 \mathrm{~mm}(0.024$ inch $)$

Nominal thickness of Side $2 \mathrm{Al}$ plate $=1.0 \mathrm{~mm}(0.039 \mathrm{inch})$, with pocket milled for foils

Friction Bonding (FB) information

FB performed on milling machine: $\quad$ Kearney \& Trekker Corp., Milwaukee, WI, Model No. 330,

No.3-30hp DP TF Series, Serial No. 7-8118

FB tool speed $=406$ RPM

FB feed rate $=94 \mathrm{~cm} / \mathrm{min}(37 \mathrm{inch} / \mathrm{min})$

FB target tool load $=48,900 \mathrm{~N}(11,000 \mathrm{lbf})$

\begin{tabular}{|l|l|l|l|}
\hline FB date & $2-28-07$ & $3-1-07$ & $2-23-07$ \\
\hline FB tool face material & Steel-A36 & Aluminum-Bronze & Anviloy \\
\hline Plate Sets & Figure 4.6.b & Figure 4.6.c \\
\hline $\begin{array}{c}\text { Prior to cutting sample: } \\
\text { photo of marked plate, } \\
\text { UT scan, and radiograph }\end{array}$ & Figure 4.6.a & & \\
\hline
\end{tabular}

a. Foil cleaning instructions:

- De-burr the foil as needed, note the sandpaper used.

- Clean the foil using 30-50\% nitric acid until any oxidation has been removed and the foil is clean.

- $\quad$ Rinse the foil with de-ionized water

- Give the foil an ethanol wipe-down.

- $\quad$ Note foil thickness. 


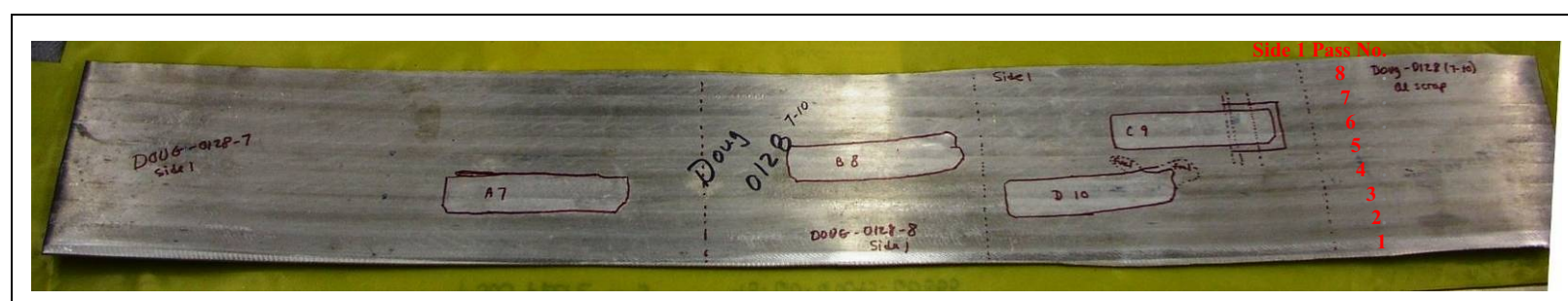

a. Side 1 marked prior to cutting sample

b. UT of Side 2
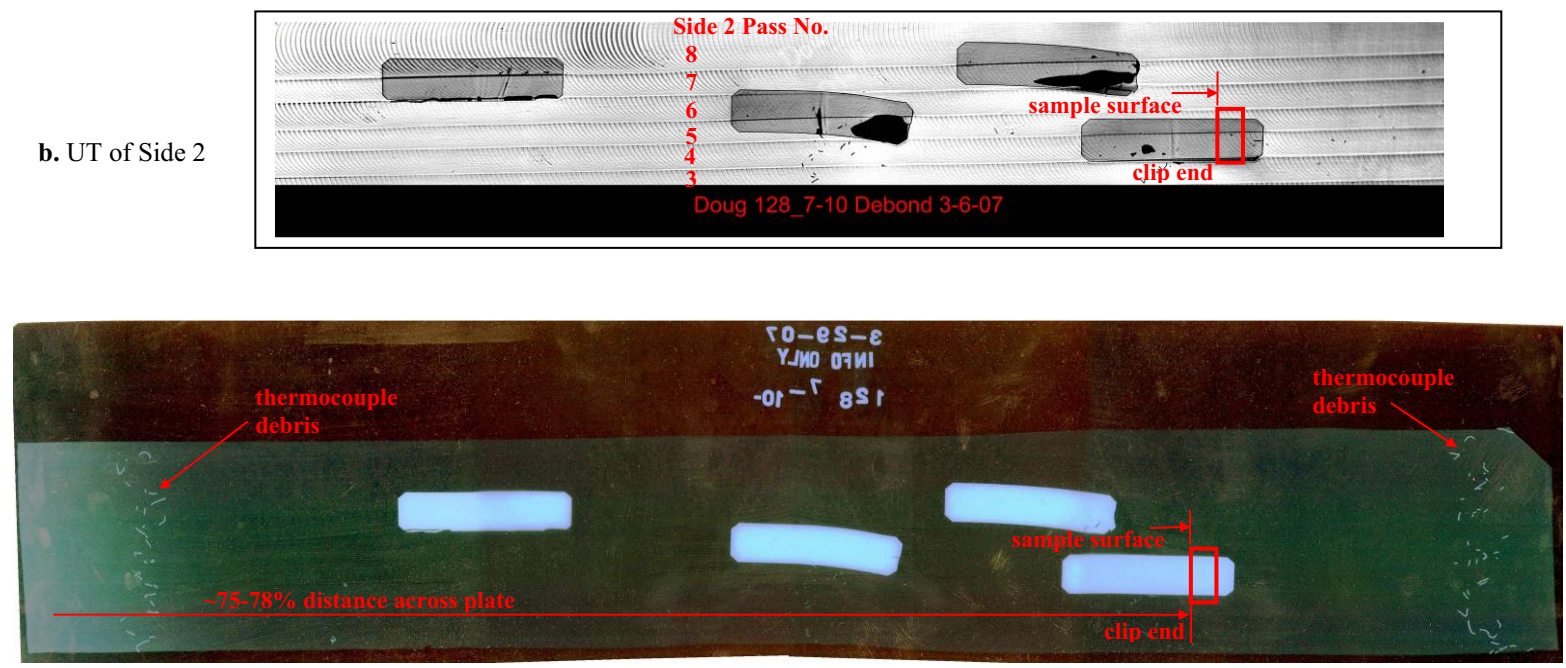

c. Radiograph of Side 2

Figure 4.6 Photos of marked plates, debond-UT scans, and x-ray radiographs for the three plate sets fabricated by friction bonding. a. FB Tool Face of Steel-A36 was used to bond DU10Mo foils inside aluminum Al-6061 cladding. 


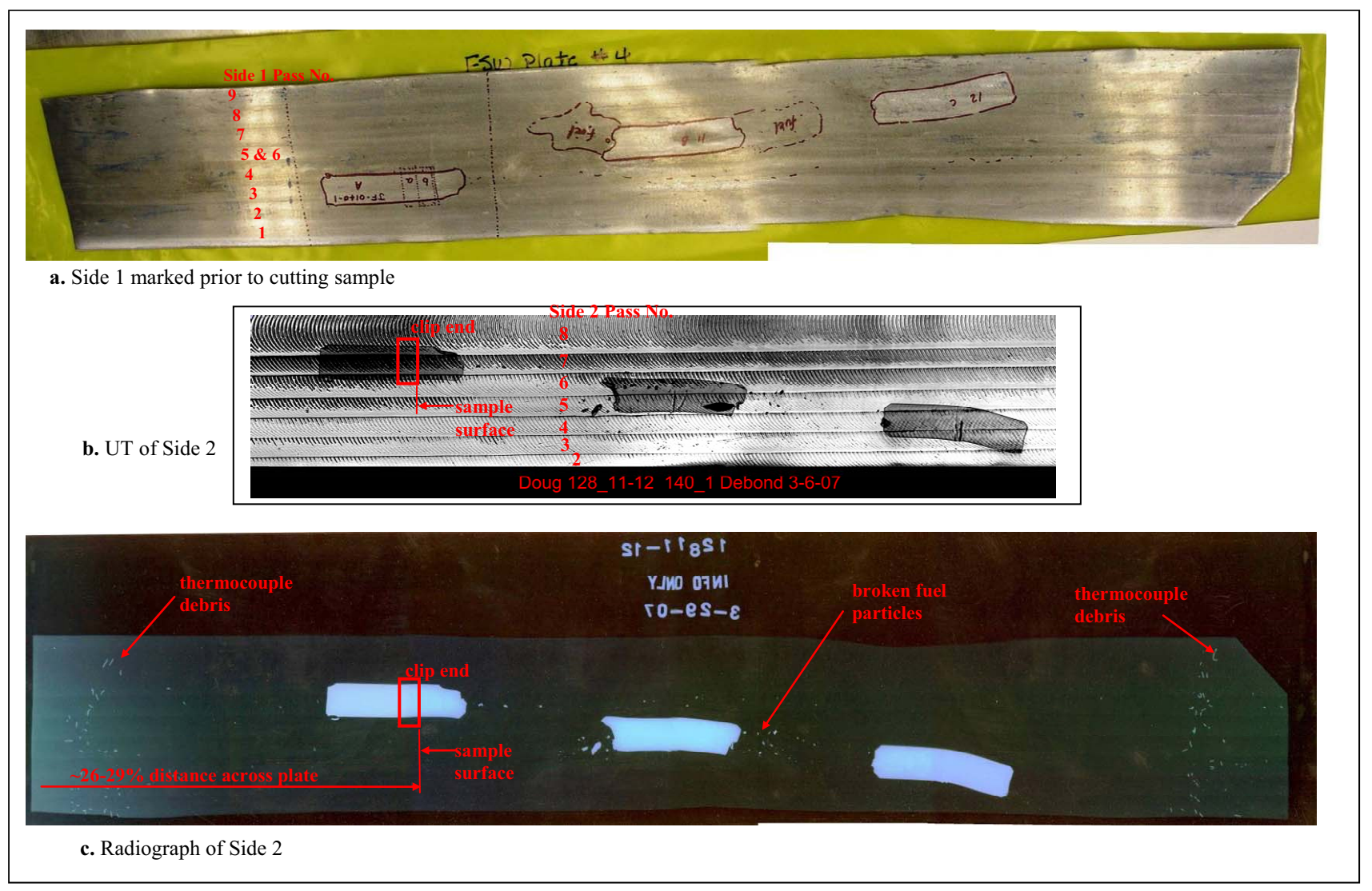

Figure 4.6.b. FB Tool Face of aluminum-bronze alloy was used to bond DU10Mo foils inside aluminum Al-6061 cladding. 


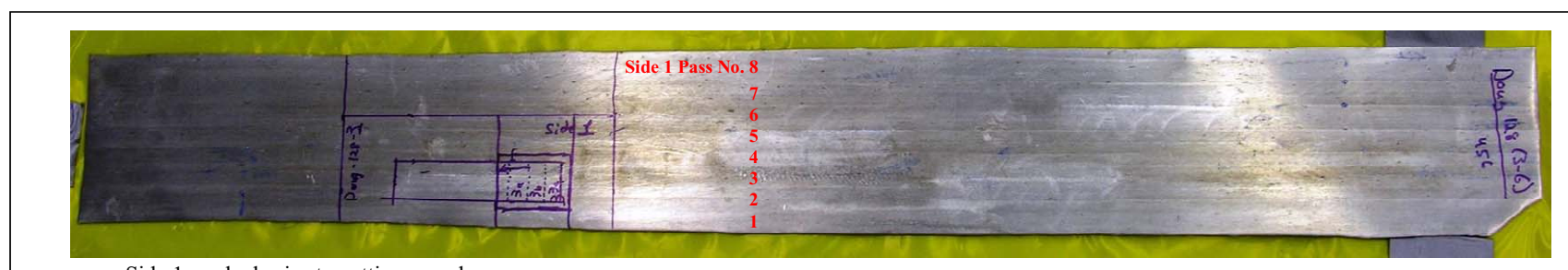

a. Side 1 marked prior to cutting sample

b. UT of Side 2

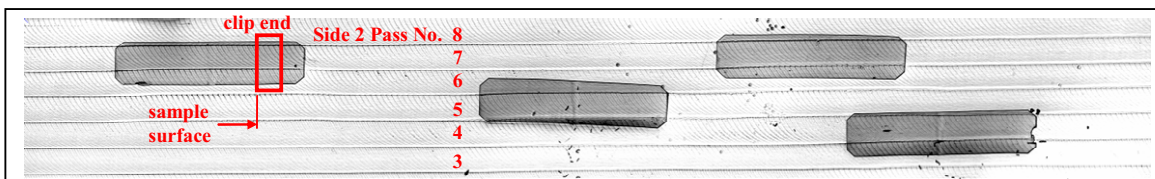

Doug 128_3-6 Debond 3-6-07
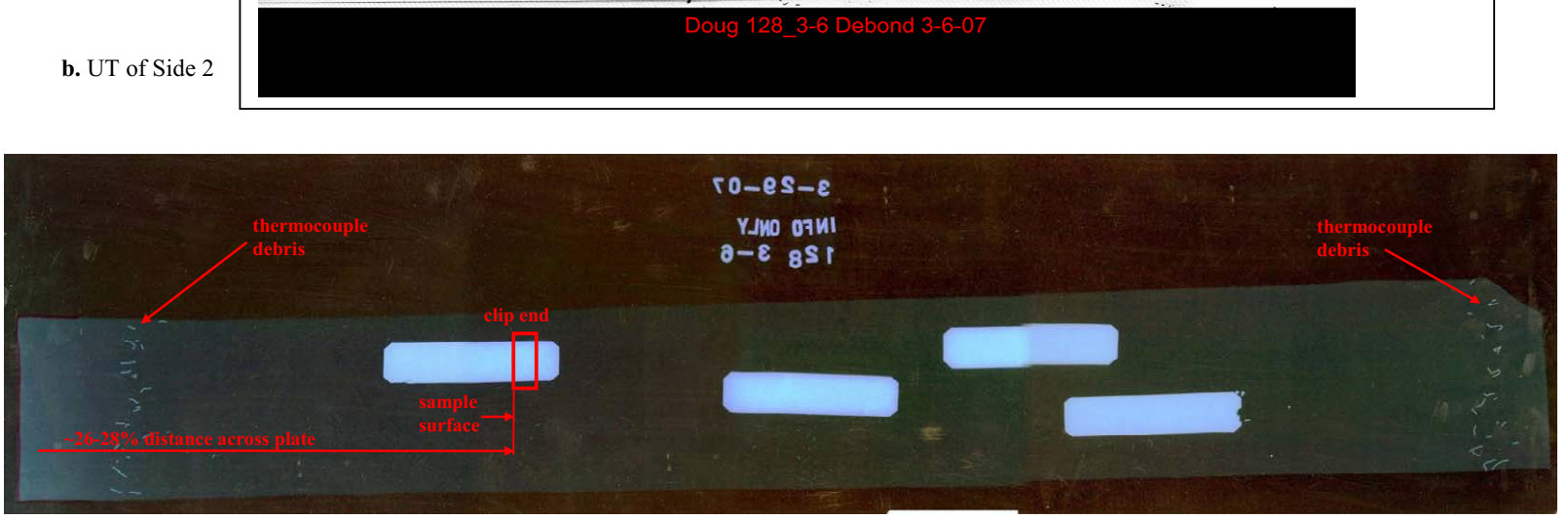

c. Radiograph of Side 2

Figure 4.6.c. Tool Face of tungsten alloy "Anviloy" was used to bond DU10Mo foils inside aluminum Al-6061 cladding. 
Tool load and temperature data gathered during FB fabrication of all the three plate sets were used to estimate relative tool work input (W) and coolant heat output (Q) for each FB pass. The calculated values are relative to each other (rather than absolute values) since some factors were constant for all the calculations and therefore were not included. Calculations were performed as follows:

a. The relative work input (W) calculation was based on the average tool load during each FB pass. Work input from rotation of the tool was neglected for this calculation, since it was constant for FB with all these tools. The calculation was: W = Average Load x Traverse Speed $x$ Pass Time Duration.

b. The relative heat output $(\mathrm{Q})$ calculation was based on the maximum change $(\Delta T)$ between tool coolant inlet and outlet temperatures during each FB pass. Based upon examination of data and curves for all the FB passes, several simplifying assumptions were justified: because the coolant outlet temperature had a similar curve shape with all tool face alloys, the maximum $\Delta \mathrm{T}$ could be used in the calculations rather than the area under the curve; heat removal lasts 1.5 times as long as work input; and an arbitrary constant value of coolant flow was assumed for FB with all the tools. Heat loss through any other avenues (e.g., convection to the air from the plate or tool, or conduction through the plate into the milling machine's anvil) is assumed approximately constant for FB with all these tools, and so was neglected for this calculation. The calculation was: $\mathrm{Q}=\operatorname{Max} \Delta \mathrm{T} \times$ Coolant Specific Heat x 1.5 x Pass Time Duration x Coolant Flow Rate.

c. Relative efficiency of the tool's heat removal from the FB process is the ratio of the relative heat output to relative work input during each FB pass. The calculation was: Efficiency $=\mathrm{Q} / \mathrm{W}$.

These measured data and calculated values for each FB pass on the three plate sets were evaluated to determine the heat removal efficiency of FB tool face alloys or any other correlations or trends that could give general insight into the friction bonding process.

\subsubsection{Samples from Specific Locations within DU10Mo Fuel Plates}

The specific fuel plate desired for sampling was selected from within each surrogate fuel plate set by perusing the debond-UT scan and examining the radiograph on the FASB light table to identify any tearing or broken fuel scattered into the aluminum by the FB process; such locations were avoided. A fuel plate was selected based on the apparent quality of its claddingfoil bond, along with its foil's smoothness and intact and straight edges. Each fuel plate's cross-section sample location was then selected to provide good representation of the claddingfoil and cladding-cladding bonds. Thus, cross section locations were not selected where a foil deformed or tore during FB fabrication, or where broken fuel pieces were nearby. Acceptable and unacceptable foil candidates and potential locations for sample cross-sections are evident in the plate sets shown in Figure 4.6. Whichever plates were selected and sectioned, the remaining portions were stored, to be available for mechanical testing to be performed under a separate characterization effort if desired. From each of the two RERTR-9A archive sets, at least one relatively good quality (foil with minimal deformation or tearing) surrogate plate was left uncut for continued archive storage.

The three FB DU10Mo samples were prepared and examined over a seven-month period. Figure 4.6 shows photographs of each plate set marked prior to cutting the samples. Monikers "Steel," "Al-Bronze," and "Anviloy" were subsequently used to identify the samples as noted in Table 4.1. After being cut from the plates, the samples were met-mounted as shown in Figure 4.7, with orientation depicted in Figure 4.8. 


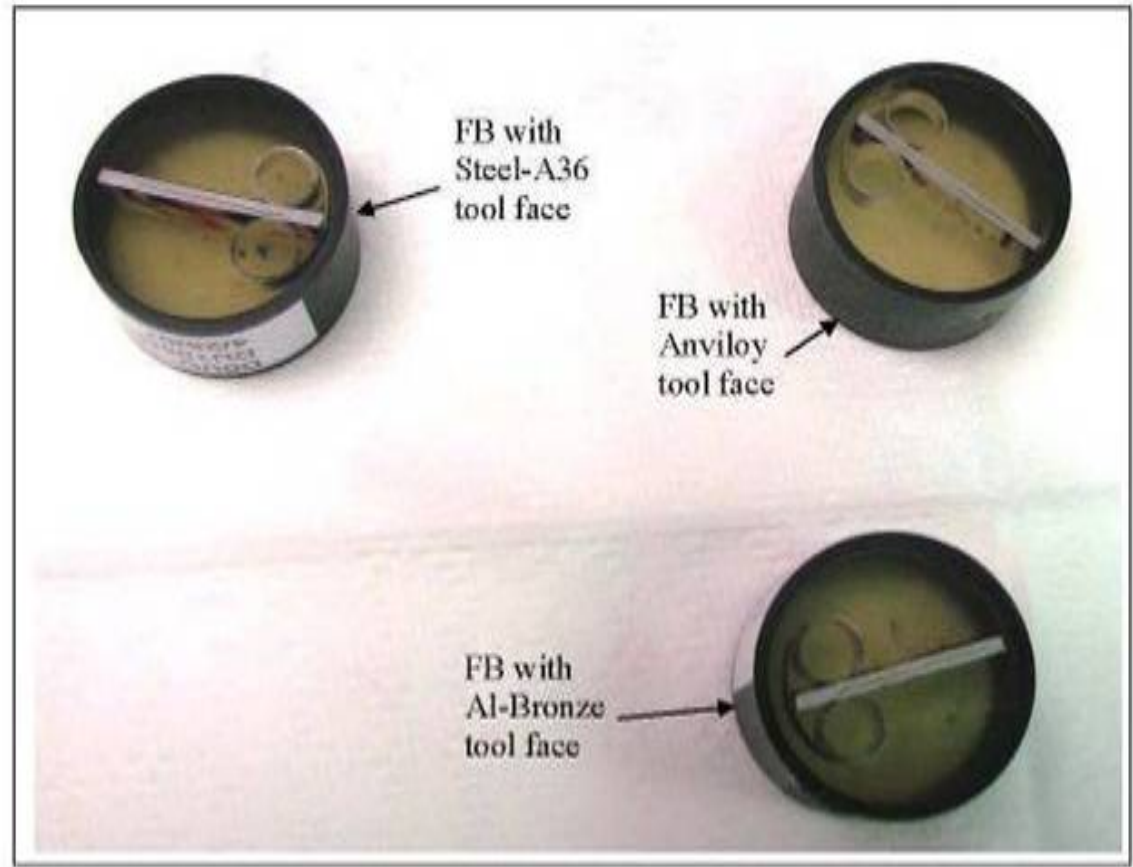

Figure 4.7 Mounted cross-sectioned samples of FB DU10Mo monolithic fuel plates, bonded with FB tools faced with different alloys. 


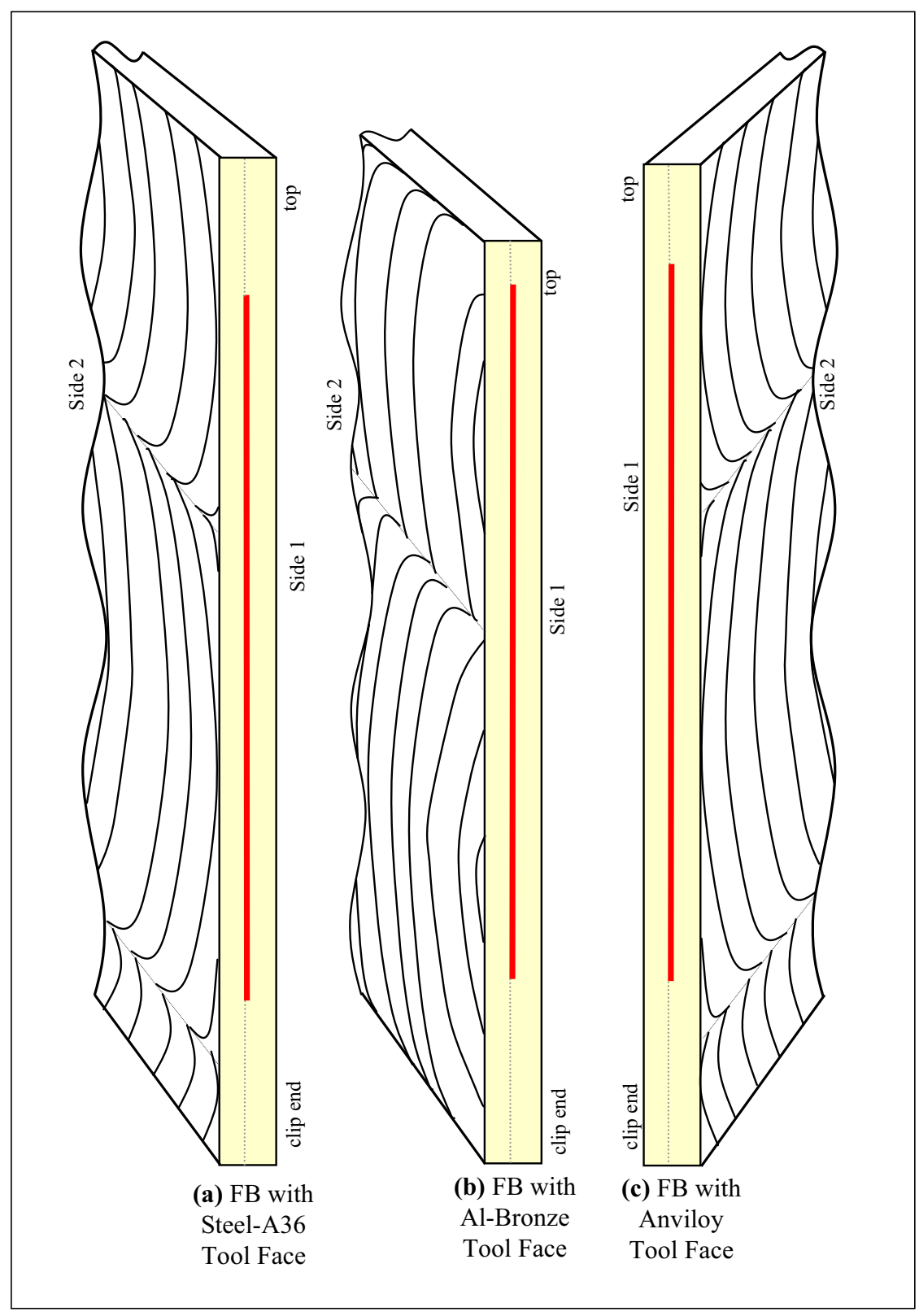

Figure 4.8 Orientation of DU10Mo fuel plate samples mounted in clips.

Note that sample (b) shows the other side of a cut from samples (a) and (c), i.e., its FB direction is opposite. Sample (c) is cut similarly to sample (a), but was rotated $180^{\circ}$ before it was mounted in its clip. FB pass seams are shown for illustration only and do not represent actual locations on each sample. 
The cross-section sample surfaces were polished and electrochemically etched with reagent comprised of $10 \mathrm{wt} \%$ oxalic acid in water, with $3 \mathrm{~V} \mathrm{AC}$ applied to the foil. Microphotographs ${ }^{14}$ were taken (Olympus n.d.) and examined. Grains and bond lines were visually examined and evaluated.

The samples were then re-polished to remove the etching, and hardness measurements were taken across each sample thickness in three cross-wise locations, and along the width of each uranium foil at a total of seven locations. At all cross-wise locations, nine hardness measurements were taken across the sample thickness. See Figure 4.9 for illustration of hardness measurement locations.

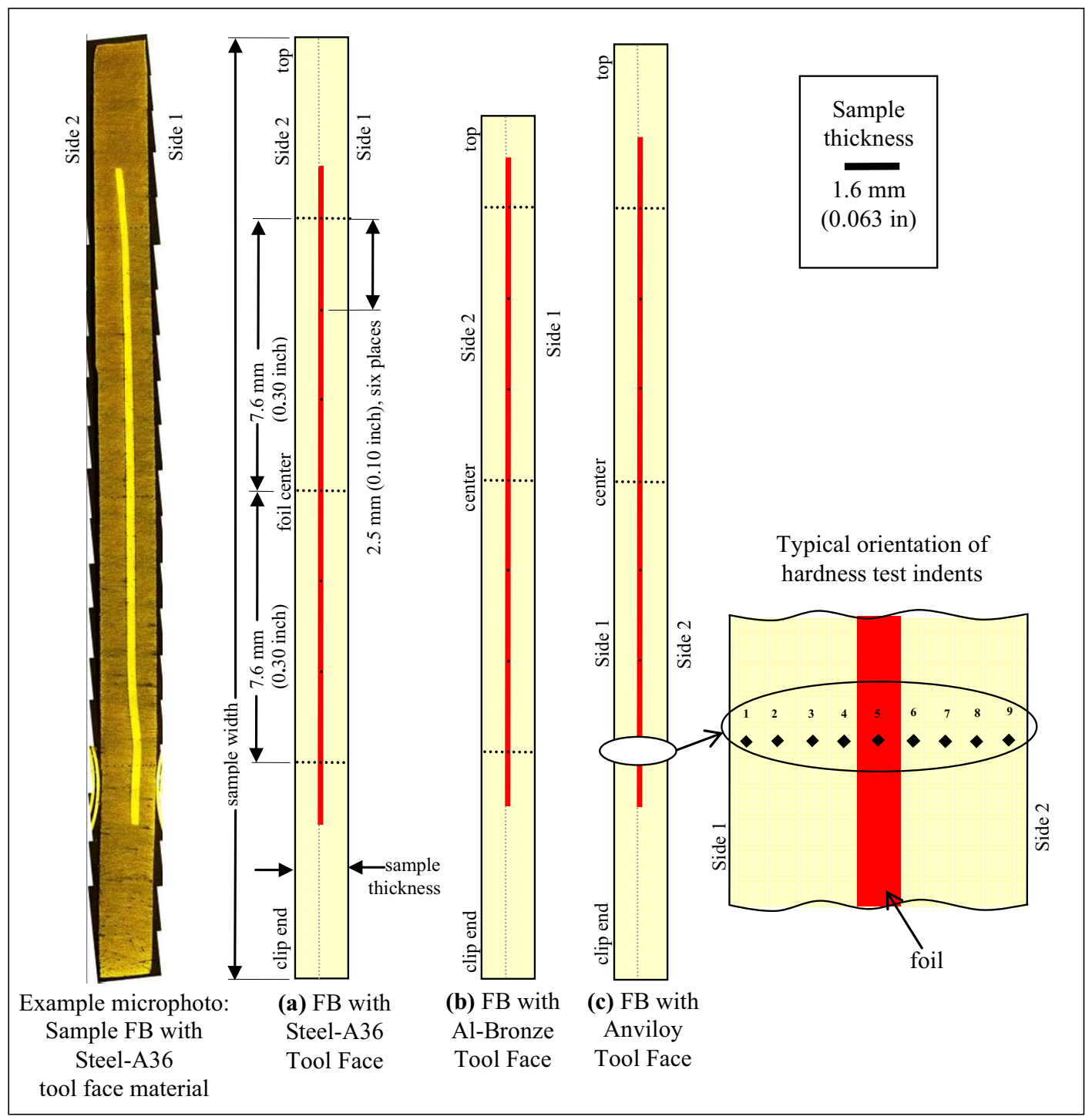

Figure 4.9 Location of Vickers Hardness tests on cross-sectioned DU10Mo surrogate fuel sample surfaces (to scale).

\footnotetext{
${ }^{14}$ Due to microscope optics and photography software, microphotographs of the DU10Mo samples show reversed "mirror" image from actual sample appearance. This section's figures with schematics of the DU10Mo samples depict sample orientation to match the microphotographs.
} 


\subsection{RESULTS AND DISCUSSION}

\subsection{What to Look For and Why}

Sample examination results were evaluated with the goal of understanding what happens during FB, i.e., the aluminum plasticizes, extrudes, and forms bonds, but exactly how does all this occur with the RERTR fuel plate materials and with the FB fabrication parameters used at the INL? What are the resulting changes in material grains, hardness, interface reactions, or other characteristics? Differences or similarities observed between the samples were interpreted as symptoms of effects of FB processing parameters. The samples were examined to observe characteristics such as:

- Sample width and thicknesses, and thickness of component layers

- Bonding or delamination between material layers - percent bonding was estimated by visual examination of grain growth across the bond line throughout each sample, viewed at 50X magnification

- Bond phenomena (for example, presence of chemical interaction layers)

- Material homogeneity, grain size, direction, and shape

- Material hardness

- Evidence of thermal effects on the materials (might be manifested by changes in any of the above characteristics).

No uncertainty analysis was performed on the examination results because there was only one sample to represent each of the varied fabrication parameters, and it is not valid to perform an uncertainty analysis on a sample size of one. However, some statistics on the sets of hardness measurements are reported in Appendix E.

Results of these microstructural examinations were evaluated in light of what is known about microstructure of samples created by FSW and FSP and how those processes compare with FB. FB is a new process, differing significantly from FSW and FSP. For example, FSW's purpose is to bond two adjoining materials by mixing - welding - them together, and FSP's purpose is to change the microstructure and properties of a material surface. By comparison, FB's purpose is to bond - but not mix - two dissimilar materials. Another difference is that FB fuel plates and their components are much thinner than plates used during FSW. The fuel plate layers are so thin and the pin so short, it is thought that no true nugget zone forms. Unlike FSW, the strength of the actual materials aren't of high concern for FB since they are obviously so much stronger than the bond. Instead the FB bond strength is of great concern and is certainly weaker than FSW bonds. Formation of a reaction layer at the U-Al interface is an issue for monolithic fuel fabrication via FB, but no such concern exists for FSW where the joined materials are typically more similar and compatible. The FB process is performed on both sides of the workpieces and in overlapping passes which erase any differing effects of advancing or retreating edges. As previously discussed, it will ultimately be necessary to fabricate FB fuel plate assemblies into fuel shapes other than just a flat plate. This differs from most FSW applications, where flat plates are the norm.

Additional insight to evaluating these examination results was gained from RERTR dispersion fuel studies as well as other RERTR studies of FB monolithic fuel. For example, because chemical interaction has frequently been observed at the foil-cladding interface as described in Appendix A, it was important to inspect this study's samples for evidence of such interaction. Temperature data gathered during prior studies helped in understanding the cause 
of some material properties observed in these samples. Previous experience with centerline bond shift during FB, and with typical symptoms of too-hot or too-cold FB material extrusions were also helpful in evaluating these samples.

\subsection{Results and Discussion of Examination of Aluminum Samples Fabricated with Varying Friction Bonding Tool Loads}

\subsubsection{Results}

These one-sided, single-pass FB aluminum samples were evaluated with knowledge that many characteristics observed here are typically wiped out on an actual fuel plate by multiple FB passes on both sides, or by radiation effects. But useful insight can be gained by evaluating the significance of those characteristics prior to their destruction.

Results of hardness measurements on these samples are listed in Table 5.1, and shown graphically in Figure 5.1 and Figure 5.2. After the samples had been re-polished, hardness measurements were repeated at a few representative locations (included in Table 5.1) These results exhibit repeatability and thus confirm validity of the previous results.

Table 5.1 Vickers Hardness measurements across aluminum samples fabricated with onesided, single-pass FB.

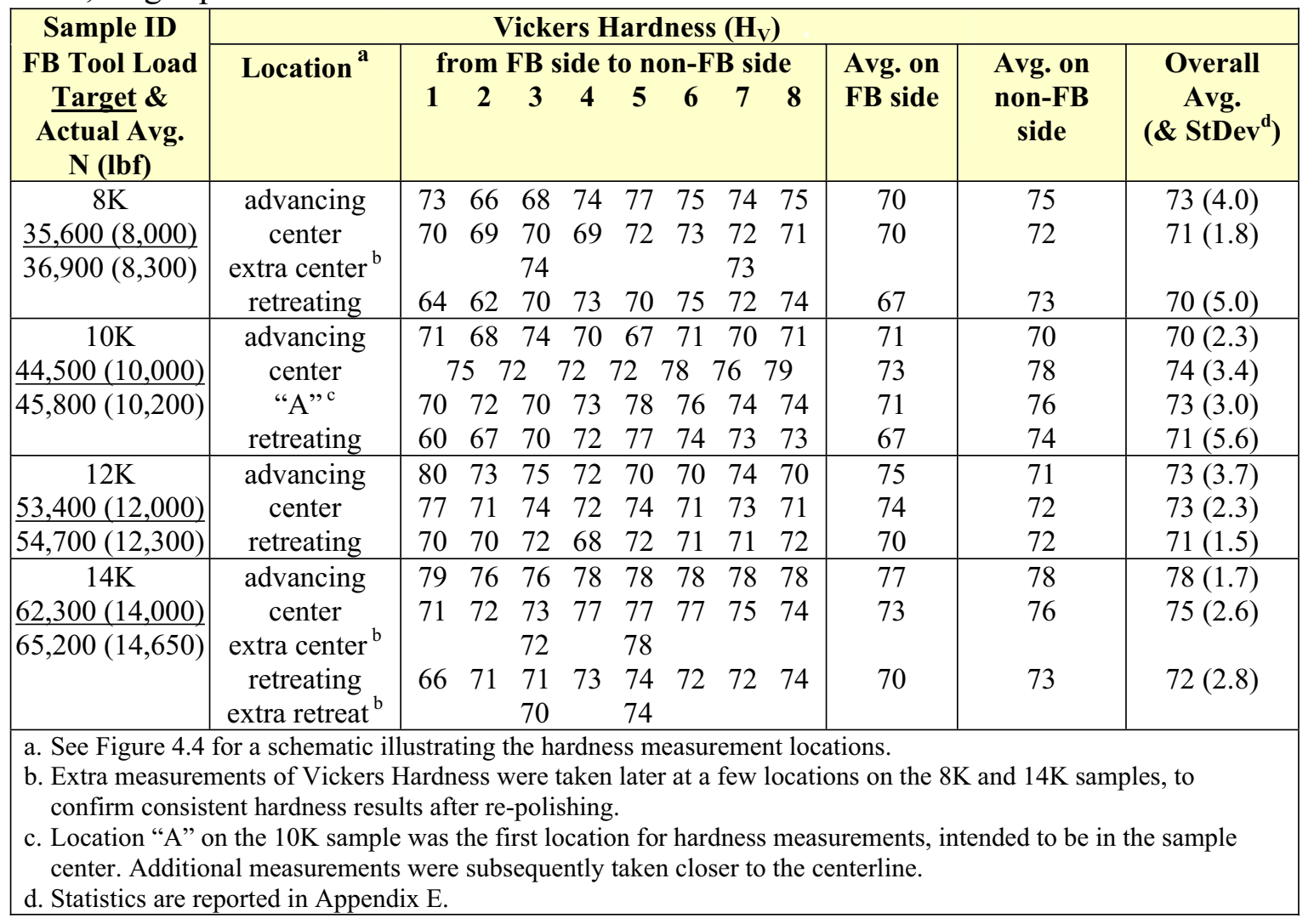



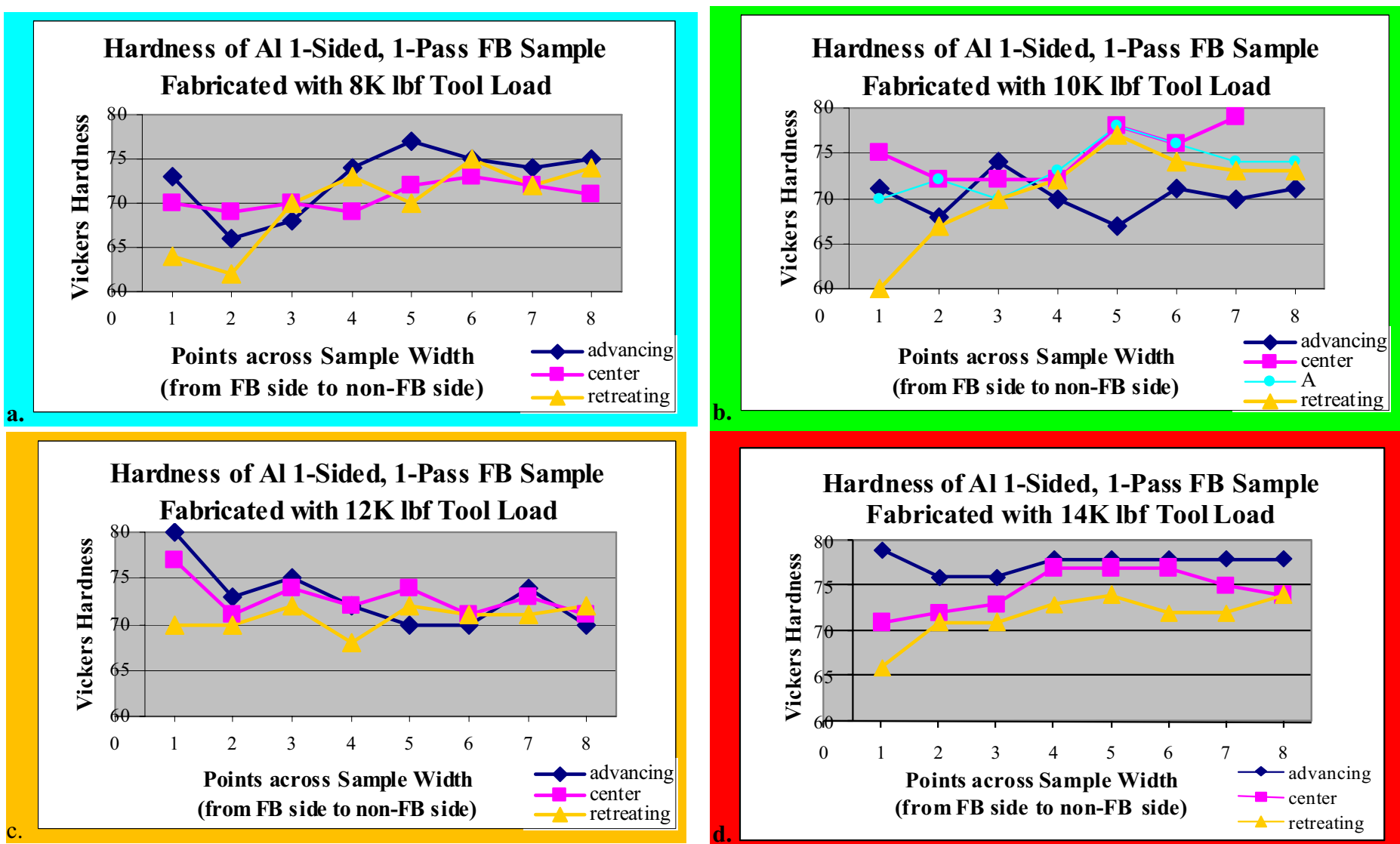

Figure 5.1 Hardness measurements on aluminum samples fabricated with one-sided, single-pass FB, with varying FB tool load of: a. 35,600 N (8,000 lbf), b. 44,500 N(10,000 lbf), c. 53,400 N (12,000 lbf), and d. 62,300 N (14,000 lbf). 

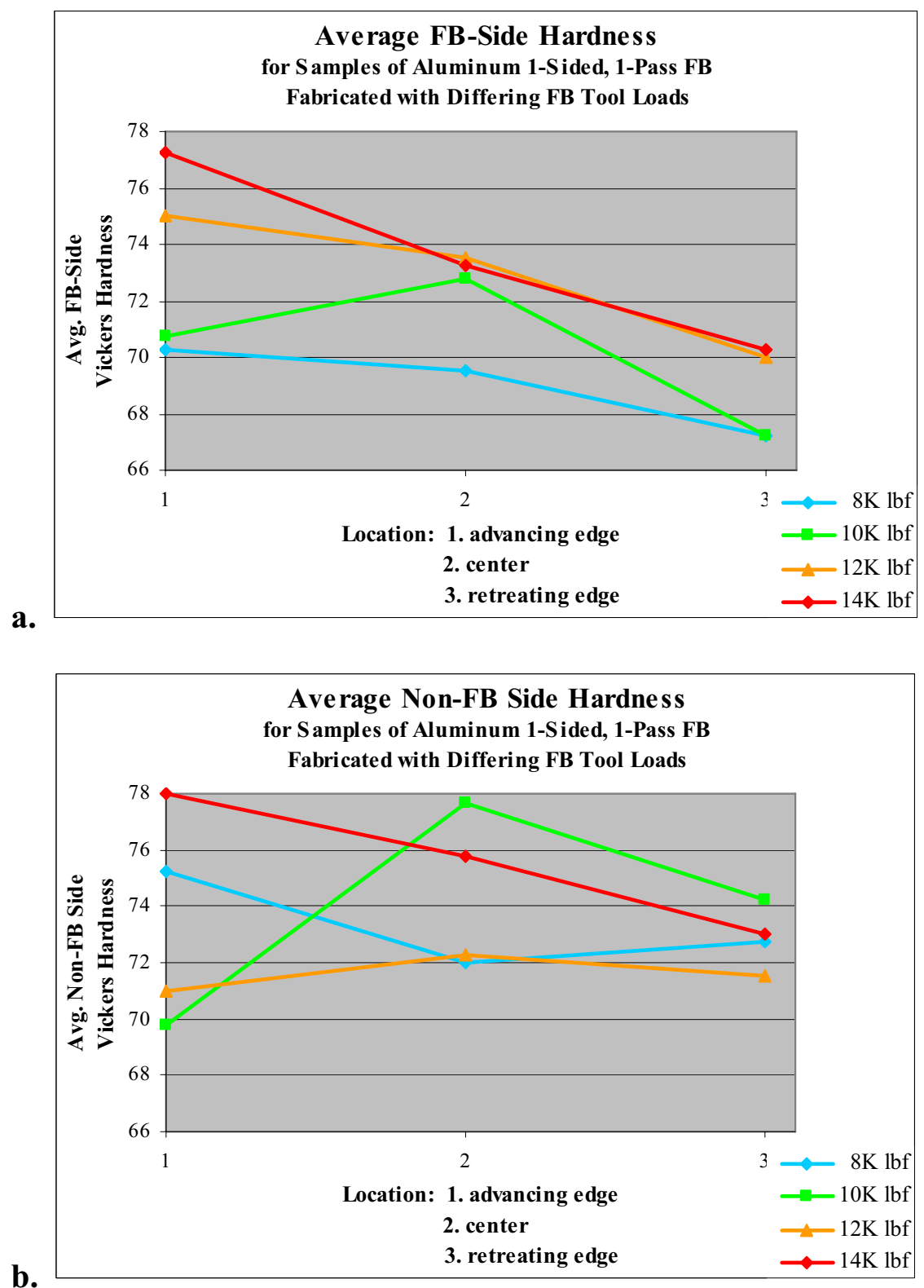

Figure 5.2 Hardness measurements on aluminum samples fabricated with one-sided, single-pass FB, with various FB tool loads.

Average hardness at: a. FB side, and b. Non-FB side. The FB side has more consistent data trends and appears more affected by the FB process than the non-FB side, where data are scattered.

All regions of the four samples were examined and microphotographed at magnifications from $12.5 \mathrm{X}$ to $1000 \mathrm{X}$. Microphotos taken at 50X were compiled into montages showing entire samples, and are provided in Figure 5.3 for all four samples side by side. Additional microphotos are given in Appendix $\mathrm{C}$ to show more detail of a few typical sample locations, chosen near some of the hardness measurement locations to allow possible correlation between the two. Table 5.2 gives bond and grain examination results gathered during microscopy. 


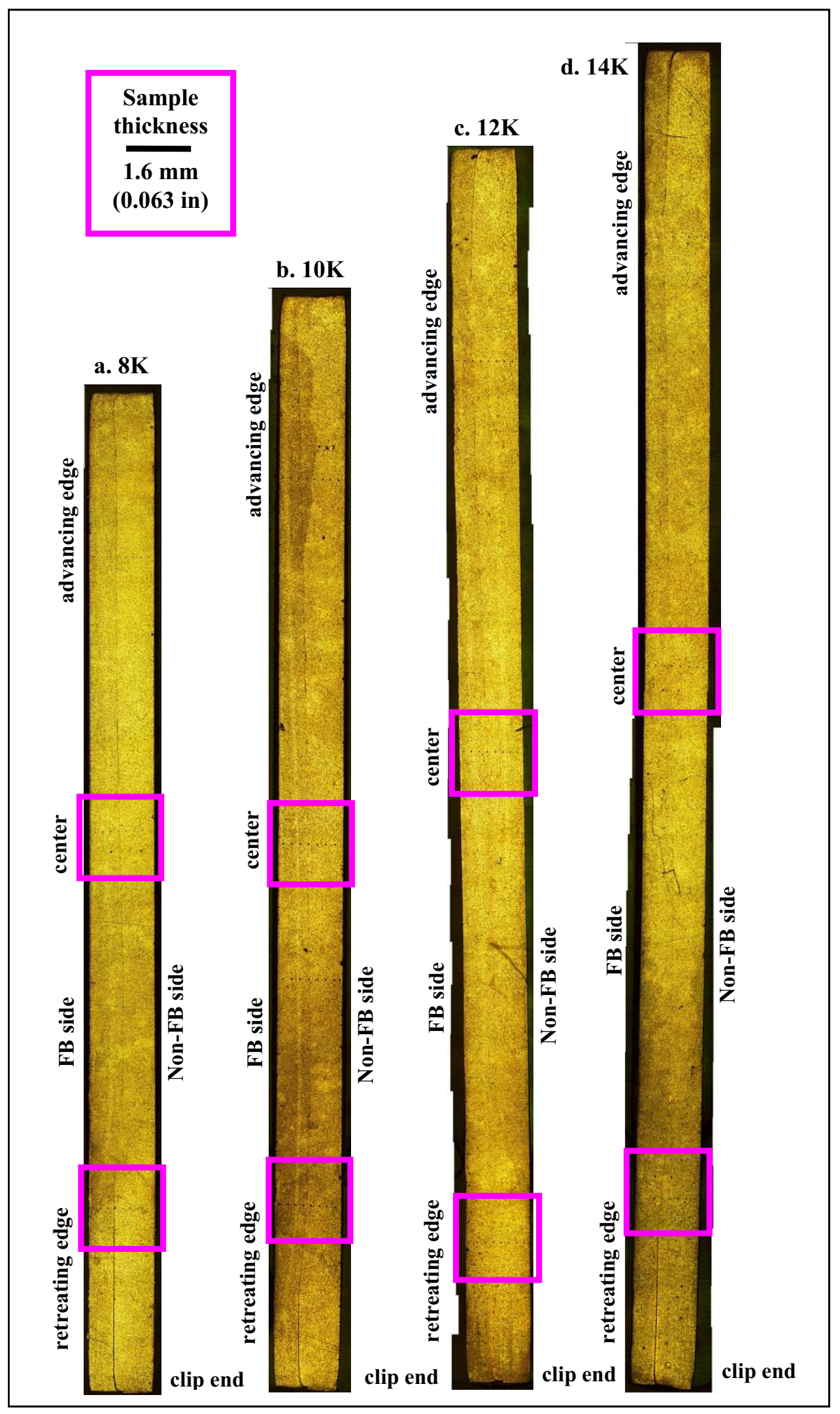

Figure 5.3 From 50X photos stitched together: Etched samples of two layers (to scale) of Al-6061(T6) aluminum joined by one-sided, single-pass FB with varying tool load of: a. $35,600 \mathrm{~N}(8,000 \mathrm{lbf})$, b. $44,500 \mathrm{~N} 10,000$ (lbf), c. $53,400 \mathrm{~N}(12,000 \mathrm{lbf})$, and d. $62,300 \mathrm{~N}(14,000 \mathrm{lbf})$. Additional microphotos of the marked locations are available in Appendix C Figures C-1 to C-4. 
Table 5.2 Estimates of bonding and grain size, shape, and direction in one-sided, single-pass FB aluminum samples fabricated with varying FB tool loads.

\begin{tabular}{|c|c|c|c|c|c|c|}
\hline \multirow[b]{2}{*}{$\begin{array}{l}\text { Target } \\
\text { FB } \\
\text { Tool } \\
\text { Load } \\
\text { N } \\
(\text { lbf) }\end{array}$} & \multirow[b]{2}{*}{$\begin{array}{l}\text { Percent } \\
\text { Bonding } \\
\text { (excluding } \\
\text { unbonded } \\
\text { ends) }\end{array}$} & \multirow[b]{2}{*}{$\begin{array}{l}\text { Location } \\
\text { in Sample }\end{array}$} & \multicolumn{2}{|c|}{ Grain Size ${ }^{a}$} & \multirow[b]{2}{*}{$\begin{array}{c}\text { Grain Shape and } \\
\text { Direction }{ }^{b}\end{array}$} & \multirow[b]{2}{*}{$\begin{array}{c}\text { Photos } \\
\text { Shown in } \\
\text { Figures } \\
\text { No. }\end{array}$} \\
\hline & & & $\begin{array}{l}\text { Avg. Area per } \\
\text { Grain }\left(\mu^{2}\right), \\
\text { measured at } \\
\text { magnification } \\
X\end{array}$ & $\begin{array}{c}\text { ASTM } \\
\text { Grain } \\
\text { Size } \\
\text { Number } \\
\text { G }\end{array}$ & & \\
\hline $\begin{array}{l}35,600 \\
(8,000)\end{array}$ & $70 \%$ & $\begin{array}{l}\text { center } \\
\text { retreating at } \\
\text { FB side } \\
\text { retreating at } \\
\text { non-FB side } \\
\end{array}$ & $\begin{array}{l}170 \\
130 \\
450\end{array}$ & $\begin{array}{l}9.6 \\
9.9 \\
8.2\end{array}$ & $\begin{array}{l}2: 1 \text { elongation, parallel } \\
\text { to sample length } \\
\text { distorted } 2: 1 \text { elongation, } \\
\text { somewhat angled }{ }^{\mathrm{b}} \\
2: 1 \text { elongation, parallel } \\
\text { to sample length } \\
\end{array}$ & $\begin{array}{r}\text { Figure } 5.3 \\
\text { \& App. C } \\
\text { Figure C-1 }\end{array}$ \\
\hline $\begin{array}{c}44,500 \\
(10,000)\end{array}$ & $85 \%$ & $\begin{array}{l}\text { center } \\
\text { retreating at } \\
\text { FB side } \\
\text { retreating at } \\
\text { non-FB side }\end{array}$ & $\begin{array}{r}170 \\
79 \\
380\end{array}$ & $\begin{array}{l}9.5 \\
10.7 \\
8.4\end{array}$ & $\begin{array}{l}\text { distorted } 2: 1 \text { elongation } \\
\text { distorted } 2: 1 \text { elongation, } \\
\text { somewhat angled }{ }^{b} \\
2: 1 \text { elongation, parallel } \\
\text { to sample length }\end{array}$ & $\begin{array}{r}\text { Figure } 5.3 \\
\text { \& App. C } \\
\text { Figure C-2 }\end{array}$ \\
\hline $\begin{array}{c}53,400 \\
(12,000)\end{array}$ & $90 \%$ & $\begin{array}{l}\text { center } \\
\text { retreating at } \\
\text { FB side } \\
\text { retreating at } \\
\text { non-FB side }\end{array}$ & $\begin{array}{l}190 \\
55 \\
220\end{array}$ & $\begin{array}{l}9.4 \\
11.2 \\
9.2\end{array}$ & $\begin{array}{l}\text { 2:1 elongation, parallel } \\
\text { to sample length } \\
\text { distorted } 2: 1 \text { elongation, } \\
\text { angled } \mathrm{b} \\
\text { 2:1 elongation, parallel } \\
\text { to sample length }\end{array}$ & $\begin{array}{r}\text { Figure } 5.3 \\
\text { \& App. C } \\
\text { Figure C-3 }\end{array}$ \\
\hline $\begin{array}{c}62,300 \\
(14,000)\end{array}$ & Over $95 \%$ & $\begin{array}{l}\text { center } \\
\text { retreating at } \\
\text { FB side } \\
\text { retreating at } \\
\text { non-FB side }\end{array}$ & $\begin{array}{c}140 \\
54 \\
160\end{array}$ & $\begin{array}{c}9.8 \\
11.2 \\
9.7\end{array}$ & $\begin{array}{l}\text { 2:1 elongation, angled } \\
\text { distorted } 2: 1 \\
\text { elongation, angled }{ }^{\mathrm{b}} \\
\text { 2:1 elongation, parallel } \\
\text { to sample length }\end{array}$ & $\begin{array}{r}\text { Figure } 5.3 \\
\text { \& App. C } \\
\text { Figure C-4 }\end{array}$ \\
\hline $\begin{array}{l}\text { a. Averag } \\
\text { two or } \\
\text { method } \\
\text { b. Grains } \\
\text { from th } \\
\text { shape, }\end{array}$ & $\begin{array}{l}\text { eating edge } \\
\text { irection. }\end{array}$ & ibit signitic & e distortion & measu & generally exhibit. & $\begin{array}{l}\text { verage of } \\
\text { at this } \\
\text { tly angled } \\
\text { in size. }\end{array}$ \\
\hline
\end{tabular}

\subsubsection{Comparison with Typical FSW and FSP Material Effects}

Evaluation of the samples' hardness measurements and microscopy examination revealed that the FB process produces workpieces exhibiting characteristics similar to those of FSW and FSP. Phenomena are consistent with phenomena observed in previously described FSW and FSP studies, and with microstructural examinations and temperature data gathered during other INL FB studies previously described. Thus, it is clear that understanding of FSW and FSP material movement and temperature effects translates well to the FB process. Since high quality FSW welds can be obtained for a wide range of fabrication parameter combinations, this bodes well for the robustness of the FB process. The conclusion of good correlation between effects of FSW/FSP and those of FB is supported by the following observations.

Average overall hardness measurements range from $70 \mathrm{H}_{\mathrm{V}}$ to $78 \mathrm{H}_{\mathrm{V}}$, about a $30 \%$ decrease from the Al-6061-T6 standard hardness of $107 \mathrm{H}_{\mathrm{V}}$. This decrease is consistent with hardness reduction that typically occurs due to dynamic recrystallization and/or dissolution of precipitates during FSW. The idea of precipitate dissolution is further supported by the fact that these hardness values are comparable to standard hardness of Al6061-T4 $[\mathrm{Hv}=75$ per 
(ASM 1990)], which is naturally aged and so has fewer precipitates than pre-FB Al6061-T6, which is tempered at higher than room temperature. The samples' hardness was measured about 7-10 weeks after FB was performed on the plates, so natural aging had likely occurred and hardness values were higher than they would have been if measured shortly after the FB was performed.

Within all the samples, grains are finer and hardness is generally lower on the FB sides than on the non-FB sides. The fine, soft grains are characteristic of TMAZ or weld arm zones, and are consistent with reduced grain size and hardness that typically occurs due to dynamic recrystallization and dissolution of precipitates during FSW.

Within all the samples, grains are finest and their hardness generally least at the retreatingedge locations; hardness is generally highest at the advancing-edge location. This trend is consistent with previously described reports of lowest temperatures occurring at the retreating edge during FB, and with previous explanation that lower temperatures during FSW typically allow less grain growth and less tempering to occur in the recrystallized grains, so that hardness is lower in these fine grains.

Close examination of Figure 5.3 reveals faint light-colored streaks running along the sample lengths, forming shallow basins that roughly parallel the bond lines. This is most pronounced in the $10 \mathrm{~K}$ sample, but also somewhat visible in other samples. In the $12 \mathrm{~K}$ sample, two streaks are visible, one on each side of the bond line. These basin-shaped streaks, although not formed in concentric rings, might correspond to the benign "onion rings" commonly reported in FSW samples. Alternatively, the streaks could correspond to the slight delineation, possibly a weld-arm manifestation, visible in the very top surface of Figure 2.3.c. Either of these explanations would indicate that the streaks' implications for fuel plate material properties are acceptable.

\subsubsection{Effects of Increased Tool Load}

Samples produced with increased FB tool load exhibit characteristics of increased work input and associated higher temperatures and forging. This conclusion is supported by the following observed impacts of increased tool load.

The FB path gets wider (samples dimensionally are wider) as tool load is increased. This is to be expected since the increased tool load increases the work input, thus causing temperature effects, cold work, forging, and bonding to occur over a wider path. Photo montages in Figure 5.3 are arranged with the retreating edges together, and the increased width shown on the advancing edge. This direction of width growth is suggested by the bond shift illustrated in Figure 2.11.A and Figure 4.2, where the asymmetrical temperature distribution across the FB tool centerline causes more bonding to occur on the hotter advancing edge than on the cooler retreating edge. (Note that "center" labels on Figure 4.4 and Figure 5.3 indicate the sample center, not the FB tool centerline.)

Increased tool load generally corresponds with decreased grain size and increased hardness. This is because the increased work input causes more plastic strain, yielding smaller-sized recrystallized grains. The higher work input also causes higher temperatures that allow more tempering (i.e. reforming of hardening precipitates) within those fine grains. As explained previously, hardness of precipitation-hardenable aluminum alloys (like A16061) depends more strongly on precipitates than on grain size, and the volume fraction, size, and distribution of the precipitates are remarkably affected by temperature (Sato, Kokawa, et al. 2001). 
The bond line between the aluminum plates becomes less visible and percent bonding increases as tool load is increased. The bonding estimates listed in Table 5.2 did not include evaluation at the sample edges, where bonding generally did not occur. Note that bonding is good in all samples, but the bond line remains visible due to typical etching effects at the interface. No evidence of Al-Al interaction layer formation was visible; none was expected at this same-material interface.

Even though the percent bonding varied with the FB tool load, all bonds are considered to be adequate as they are all greater than the RERTR's rule-of-thumb threshold of $50 \%$ bonding. In addition, it is important to realize that the bonding observed in these samples is not representative of the bonding in actual fuel plates, which will have even more bonding due to overlapping FB passes performed on both sides. Therefore bonding observed in all these samples predict successful cladding-cladding bonding for an actual fuel plate using any of the tested FB tool loads.

\subsubsection{Microstructure Differences depending on Location in Sample}

Examination of the previous tables and figures reveals that all the samples exhibit significant differences in phenomena observed between FB sides and non-FB sides and between advancing and retreating edges. This leads to the conclusion that, if homogeneous material properties are desired in fuel plates, then current FB practices should be continued, i.e. overlapping FB passes, and FB performance on both sides of the assembly.

It can also be concluded that aluminum plasticization during FB is a key driver of material properties and sample characteristics. Thus FB process variables that impact aluminum plasticization via material movement and temperature are important to determining those characteristics; differences between sample FB and non-FB sides and between advancing and retreating edges result from differences in these process variables. As noted previously, the effects of heat generation and material movement by the FB process involve many variables and by nature are complex and hard to predict. Thus causes of some of the observed differences are not obvious and are explained here.

\section{a. Differences between Sample Sides}

Hardness and grain phenomena and trends discussed above are exhibited more consistently on the samples' FB sides than on the non-FB sides. The FB side has more grain distortion with softer, finer, and less evenly shaped grains than the non-FB side. These differences are especially pronounced at the retreating-edges, ${ }^{15}$ where grain size and hardness variations across the sample thickness are greatest.

It is noteworthy that FB effects are apparent throughout the sample: even on the non-FB side grains are smaller and softer than base material. This is true everywhere, including at the retreating edge where there isn't even a bond across the aluminum layers. As the FB tool load increased, the grain-size decreased at this location although the aluminum layers were still not bonded. The hardness at this location did not change significantly as tool load increased.

Although temperatures were higher on the FB surface, grains at that location are finer and softer. This is opposite to results of many FSW studies, where the coarsest and hardest grains are found near the workpiece surface where high temperatures yield grain growth and tempering. But in these samples, because of the very small pin extension into the material, the

\footnotetext{
${ }^{15}$ Grains at the retreating-edge locations can be observed in Appendix C Figures C-1.f, C-2.e, C-3.f, and C-4.f.
} 
FB side experienced considerably more work strain than the non-FB side, with effects such as disrupted grain boundaries and finer and softer grains. It appears the higher temperatures at the FB surface were not high enough to counter these effects, i.e., to cause the grains to grow or temper enough to regain size or hardness equal to grains below the surface. Temperatures further from the FB surface were lower, but grains are coarser and generally not as soft because they experienced less work strain effects.

Grains in all samples are consistently elongated with approximate ratio of $2: 1$, although grains at some locations exhibit significant shape distortion. Direction of elongation, presence of distortion, and grain size vary through each sample in similar ways. For example, at the retreating edge in all samples, grains on the non-FB side are consistently parallel to the sample length, and grains on the FB side are distorted and angled from the retreating edge toward the sample center, consistent with TMAZ grain orientation around the weld center. Grains in the sample center are either not strongly angled, or their direction differed randomly from one sample to another; this is consistent with their location closer to the tool centerline.

\section{b. Differences between Sample Edges}

All samples have reduced bond at the advancing edge, and no bond at the retreating edge, probably due to a lower forging effect near the tool edges. ${ }^{16}$ The lack of bonding and presence of the coarsest grains at the retreating-edge non-FB side give evidence that the lowesttemperature and least-affected location in all samples was the retreating-edge non-FB side. This is consistent with temperature data reported in other studies previously described.

All samples have their finest grains and lowest hardness on the retreating-edge FB side. Grain size and hardness variations across the sample thickness are greatest at the retreating edge, with very fine soft grains found at the FB side and coarse harder grains at the non-FB side. Grains at other locations generally appear to be sized between these extremes. ${ }^{17}$

These characteristics at the samples' retreating edge - lack of bonding; soft, distorted, and super-fine grains on the FB side; and a larger variation in grain size across the sample thickness - are all symptoms of the retreating edge's lower temperature compared with the rest of the sample. As previously explained, at lower temperatures the aluminum is less plastic, so FB results in higher strain rate, more disrupted grain boundaries, and therefore finer grains. The softer grains are consistent with (Sato, Urata and Kokawa 2002)'s theory that fine grains inherently have less precipitation volume. In addition, the retreating edge's lower temperatures promote less recrystallization, grain growth, or tempering; this leaves more distorted, finer, and softer grains than other sample locations affected by higher temperatures.

Each sample has almost constant total thickness along its width, consistent with the original total thickness of the two plates together. However, the two aluminum layers, which initially had uniform thickness of $0.6 \mathrm{~mm}(0.024 \mathrm{in})$ on the FB side, and $1.0 \mathrm{~mm}(0.039 \mathrm{in})$ on the nonFB side prior to the FB process, are now changed in relative thickness as revealed by examination of the bond lines. At the retreating edge, the material thicknesses are the same as their initial thicknesses. However, in the region between the retreating edge and the sample center, the thickness of the layer on the FB side increased to about the same thickness as that

\footnotetext{
${ }^{16}$ It was also observed that all sample bond ends are bent to one side, probably due to a swaging effect of the shear during initial sample sizing; sample ends were not trimmed with the high-speed saw prior to mounting in epoxy. This observation is not significant to understanding the FB process.

${ }^{17}$ Although the coarsest grains on the $12 \mathrm{~K}$ sample appear to be located at the non-FB side of the advancing edge, the largest variation in grain size across the sample thickness still occurs at the retreating edge.
} 
on the non-FB side; this region corresponds with the most pronounced (darkest) etching and with the deepest point of the basin-shaped streaks, and is probably near the location of the FB tool centerline. Toward the samples' advancing edges, the aluminum layer on the FB side is decreased to less than its original thickness. This thickness is not as decreased in the $8 \mathrm{~K}$ sample as it is in the other three samples. In the $10 \mathrm{~K}, 12 \mathrm{~K}$, and $14 \mathrm{~K}$ samples the decreased thickness of the advancing-edge FB side layer appears about the same, i.e. not further impacted by increased tool load. These changes in the aluminum layer thicknesses are indicative of the aluminum plasticization that occurs with FB.

\subsection{Results and Discussion of Friction Bonding and Examination of DU10Mo Samples Fabricated with Varying Tool Face Alloys}

Two sets of data were gathered and evaluated on FB of DU10Mo fuel plates with varying tool face alloys. The first data set and its evaluation were related to tool loads and temperatures measured during general FB of the three entire fuel plate sets. The second was related to examination of the three samples, each one taken from a specific location within a fuel plate from each fuel plate set.

Even though the target load was the same $(48,900 \mathrm{~N}=11,000 \mathrm{lbf})$ for all FB passes on these surrogate fuel plates, the actual load varied considerably due to the art of manual control. Actual tool loads and temperatures recorded during FB of these surrogate fuel plates were evaluated and then location-specific samples were examined. These evaluations were performed to gain a more comprehensive understanding of the FB process including effects of multiple passes and FB on both sides of the plate, of the uranium alloy fuel foil's presence, and of differing FB tool face materials.

\subsubsection{Analysis of General Friction Bonding Process on DU10Mo Fuel Plate Sets}

During fabrication of the three fuel plate sets, measurements were continuously taken of the FB tool load and internal temperature, and coolant inlet and outlet temperatures. For example, Figure 5.4 shows data gathered during FB of one side of one plate set, illustrating that load fluctuations during each FB pass are of much greater variability than fluctuations in the resulting temperatures. The average FB tool load and temperature and the maximum change in tool coolant temperature measured during each FB pass on all the plate sets are given in Table 5.3. No plate temperatures were measured or recorded.

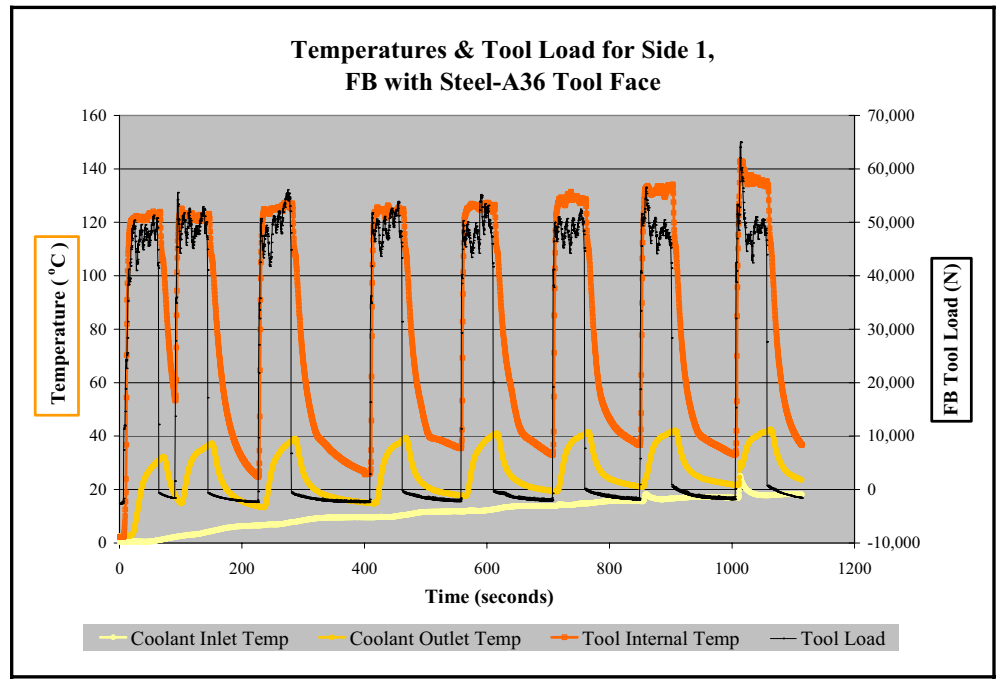

Figure 5.4 Temperature and tool load data illustrative of fluctuations observed during FB of DU10Mo monolithic fuel plate sets. 
Table 5.3 Average FB tool load and temperature, and tool coolant maximum temperature change, measured during each FB pass on DU10Mo monolithic fuel plate sets fabricated with varying FB tool face alloys.

\begin{tabular}{|c|c|c|c|c|c|c|c|c|}
\hline \multirow{2}{*}{$\begin{array}{l}\text { FB } \\
\text { Tool } \\
\text { Face } \\
\text { Alloy }\end{array}$} & \multicolumn{4}{|c|}{ Side 1} & \multicolumn{4}{|c|}{$\begin{array}{l}\text { Side } 2 \\
\end{array}$} \\
\hline & $\begin{array}{l}\text { Pass } \\
\text { No. }\end{array}$ & $\begin{array}{c}\text { Average } \\
\text { FB Tool } \\
\text { Load } \\
(\mathbf{N}) \\
\end{array}$ & \begin{tabular}{|c|} 
Average \\
FB Tool \\
Temperature \\
$\left({ }^{\mathbf{0}} \mathbf{C}\right)$ \\
\end{tabular} & $\begin{array}{c}\text { Tool Coolant } \\
\text { Inlet-Outlet } \\
\text { Maximum } \\
\Delta \text { Temp }\left({ }^{\circ} \mathrm{C}\right) \\
\end{array}$ & $\begin{array}{l}\text { Pass } \\
\text { No. }^{a}\end{array}$ & $\begin{array}{c}\text { Average } \\
\text { FB Tool } \\
\text { Load } \\
(\mathbf{N}) \\
\end{array}$ & $\begin{array}{c}\text { Average } \\
\text { FB Tool } \\
\text { Temperature }^{\text {b }} \\
\left({ }^{0} \mathrm{C}\right) \\
\end{array}$ & $\begin{array}{c}\text { Tool Coolant } \\
\text { Inlet-Outlet } \\
\text { Maximum } \\
\Delta \text { Temp }\left({ }^{\circ} \mathrm{C}\right) \\
\end{array}$ \\
\hline \multirow{8}{*}{$\begin{array}{l}\text { Steel } \\
\text { A36 }\end{array}$} & 1 & 47,477 & 122 & 30.9 & 1 & 46,880 & 137 & 27.8 \\
\hline & 2 & 49,208 & 122 & 32.8 & 2 & 47,671 & 140 & 28.6 \\
\hline & 3 & 49,596 & 125 & 31.1 & 3 & 48,818 & 140 & 28.5 \\
\hline & 4 & 49,271 & 124 & 28.8 & 4 & 48,829 & 140 & 27.9 \\
\hline & 5 & 48,787 & 126 & 28.2 & 5 & 50,534 & 140 & 27.8 \\
\hline & 6 & 48,724 & 128 & 26.7 & 6 & 51,992 & 141 & 27.2 \\
\hline & 7 & 49,030 & 132 & 25.4 & 7 & 50,388 & 143 & 26.3 \\
\hline & 8 & 49,968 & 136 & 24.6 & 8 & 53,769 & 142 & 25.5 \\
\hline \multirow{8}{*}{$\begin{array}{c}\text { Al- } \\
\text { Bronze }\end{array}$} & 1 & 47,536 & 129 & 14.9 & 1 & 47,230 & 138 & 15.7 \\
\hline & 2 & 49,445 & 130 & 17.0 & 2 & 48,999 & 139 & 17.8 \\
\hline & 3 & 50,414 & 131 & 16.9 & 3 & 48,501 & 141 & 18.3 \\
\hline & 4 & 50,805 & 132 & 17.1 & 4 & 49,238 & 141 & 18.5 \\
\hline & 5 & 50,562 & 133 & 16.8 & 5 & 50,468 & 142 & 18.4 \\
\hline & 6 & 52,386 & 135 & 16.4 & 6 & 50,228 & 142 & 18.0 \\
\hline & 7 & 50,722 & 136 & 16.5 & 7 & 46,932 & 141 & 17.7 \\
\hline & 8 & 52,905 & 138 & 16.5 & 8 & 47,830 & 141 & 17.8 \\
\hline \multirow[t]{8}{*}{ Anviloy } & 1 & 48,104 & 131 & 27.6 & 1 & 47,687 & 146 & 25.2 \\
\hline & 2 & 48,837 & 133 & 28.2 & 2 & 49,131 & 145 & 25.9 \\
\hline & 3 & 50,619 & 134 & 28.0 & 3 & 48,241 & 145 & 26.3 \\
\hline & 4 & 51,871 & 135 & 26.6 & 4 & 48,621 & 146 & 25.8 \\
\hline & 5 & 52,837 & 136 & 25.4 & 5 & 48,915 & 145 & 25.0 \\
\hline & 6 & 51,243 & 137 & 23.6 & 6 & 49,262 & 145 & 25.0 \\
\hline & 7 & 51,558 & 140 & 23.4 & 7 & 48,966 & 147 & 24.1 \\
\hline & 8 & 59,415 & 142 & 23.7 & 8 & 48,242 & 146 & 24.3 \\
\hline
\end{tabular}

\section{a. Heat Removal Efficiency of Tool Face Alloys}

As described previously, data recorded during FB of the three plate sets were used to calculate estimated relative tool work input (W), coolant heat output (Q), and the tool's heat removal efficiency (W/Q) for each FB pass; these are illustrated in Figure 5.5.

Evaluation of Figure 5.5 yields the following conclusions regarding relative efficiency of heat extraction by the various tool face alloys:

- The steel tool face exhibited the highest efficiency; it consistently removed the most heat from the FB process even with variable work input. Its internal temperature was consistently lower than that of the other tool face alloys. (It can be noted from Table 4.2 that steel has the lowest thermal conductivity and the highest specific heat with the lowest density.)

- The aluminum-bronze tool face exhibited the lowest efficiency; it removed the least heat from the FB process even with variable work input. It experienced intermediate internal temperatures. (Table 4.2 shows that aluminum-bronze has fairly low thermal conductivity, high specific heat, and low density.) 


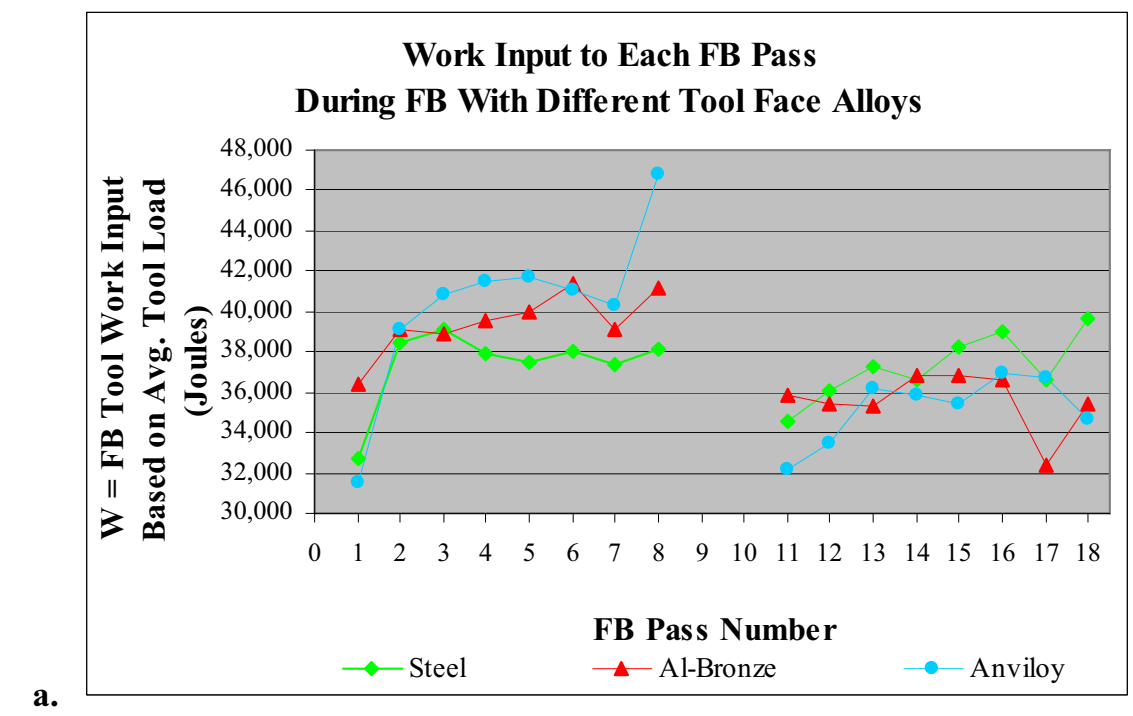

a.

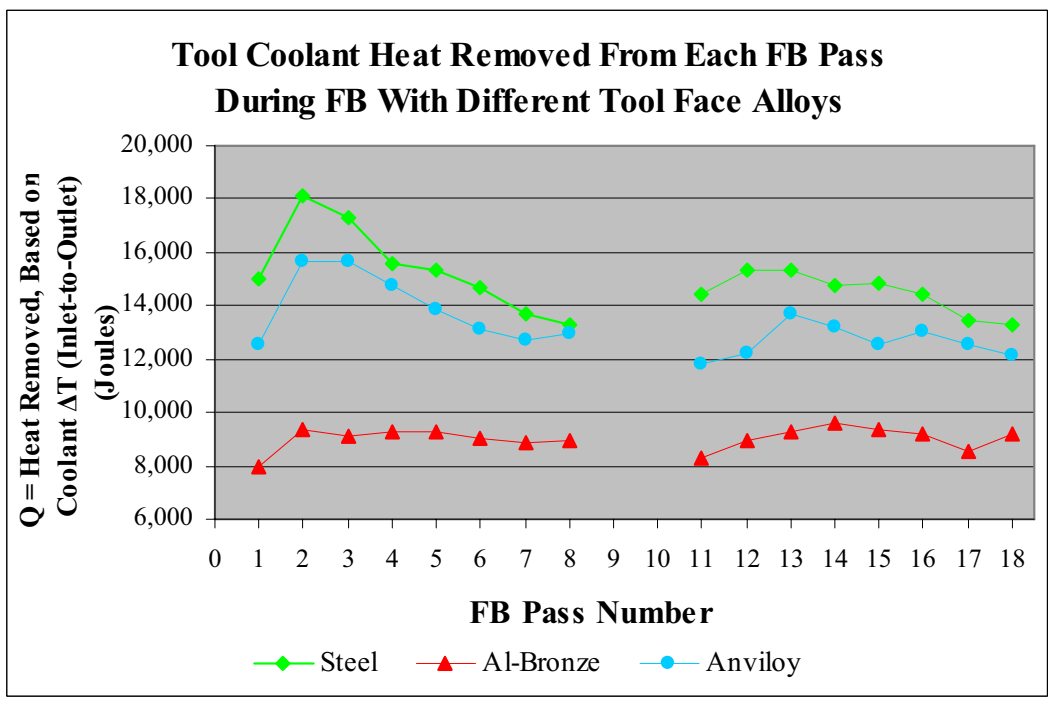

b.

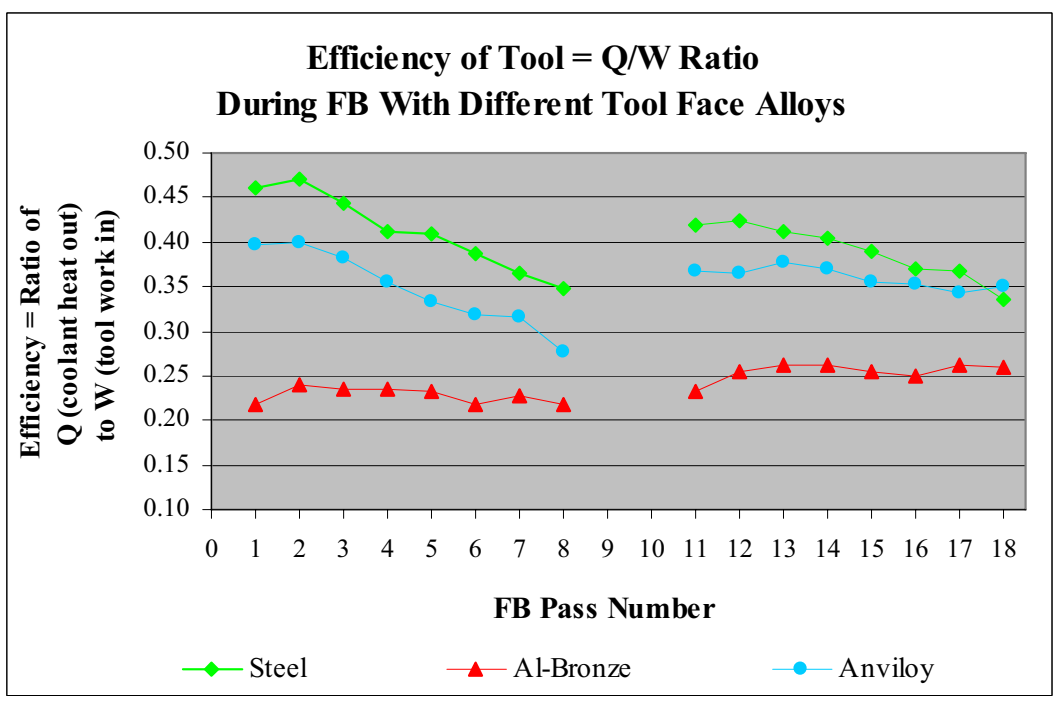

Figure 5.5 Relative tool work input and coolant heat output during each FB pass on all plate sets. Side 1 FB was performed during pass numbers 1-8, and Side 2 FB was performed during passes numbered 11-18 on these graphs. 
- The Anviloy tool face exhibited intermediate efficiency, based on intermediate amounts of heat removal from the FB process during variable work input. Its internal temperature was consistently higher than that of the other tool face alloys. (Table 4.2 indicates that Anviloy has relatively very high thermal conductivity, very low specific heat, and very high density.)

The above conclusions regarding heat extraction by the various tool face alloys are supported by observation of aluminum surface texture shown on the plate sets' UT scans in Figure 4.6.a, b, and c, as well as consideration of Figure 2.8. On the fuel plate set fabricated with FB tool face of Al-Bronze, the FB process created very rough scallops, similar to those shown in the "hot" FB illustrated in Figure 2.8.C and D. These rough scallops are probably related to the low heat removal by the Al-Bronze tool; theoretically more heat remained in the plate, causing higher plate temperatures with more aluminum plasticization and extrusion by the tool. The scallops were smoother on the Steel-A36 sample and were most smooth on the Anviloy sample.

The difference in scallop roughness between the plate sets might be amplified by differences in wear-resistance of the tool face alloys (noted in Table 4.2); the worn and galled surface of the Al-Bronze tool face probably physically stirred the aluminum more. It is possible that the high loads and thermal cycling at elevated temperatures occurring during FB hastened degradation of the Al-Bronze face, resulting in a rougher surface with higher friction, stirring the aluminum more and increasing the temperature, thus exacerbating the problematic cycle.

\section{b. Load and Temperature Trends during the Friction Bonding Process}

Additional consideration of Table 5.3 and Figure 5.5 reveals common trends for FB with all the tool face alloys:

- Tool internal temperatures generally increased strongly throughout the FB process duration for both plate sides, while the work input (W) often but not always increased, and both the coolant $\Delta \mathrm{T}$ and the heat removal $(\mathrm{Q})$ generally decreased. These trends indicate that the work input caused some cumulative increase in tool temperature that could not be removed by the coolant as quickly as it was being produced.

- Tool internal temperatures during FB of Side 2 were consistently higher (by about $4-15^{\circ} \mathrm{C}$ ) than those during FB of Side 1, in spite of generally lower work input (W) and similar heat removal (Q) during Side 2 FB when compared with Side 1. This again implies that the maximum heat removal capacity of the coolant system had been reached, with consequently higher tool temperatures.

- The FB process generally began with low work input (W) during the first FB pass, with consequent low tool internal temperatures, low coolant $\Delta \mathrm{T}$, and low heat removal (Q). This pattern was more common on Side 1 than on Side 2.

The first two trends have implications for the FB tool coolant system. It appears the coolant system capacity should be increased to adequately remove heat from the FB tool, so that constant plate and tool temperatures can be maintained during FB when all other variables are also held constant.

The reason for the third trend is not clear and is not consistent with previously described expectations for FB operations. It is speculated that the lower work input on the first pass of each side possibly resulted from the operator's practice of manual load control based on visual feedback of the aluminum plate surface during FB. However, it is not understood why a lower load would be required on the first FB pass of Side 1, when the previously untouched 
aluminum is harder and cooler. This first pass would heat and possibly soften the aluminum for subsequent passes, and it is not understood why an increased load would then be needed. The first pass of Side 2 is on aluminum that would also be cooler than subsequent passes, but would be pre-softened by the Side 1 effects. Further research is needed to understand this trend.

\subsubsection{Analysis of Samples from Specific Locations within DU10Mo Fuel Plates}

\section{a. Microscopy on Samples}

To better understand differences and similarities among the three samples, all regions of the samples were examined and microphotographed at magnifications from $12.5 \mathrm{X}$ to $1000 \mathrm{X}$. Microphotos taken at 50X were compiled into montages showing entire samples, and are provided in Figure 5.6 for all three samples side by side. Additional microphotos are provided in Appendix D to show more detail of a few typical sample locations.

The microphotos indicate success of the FB process at meeting some of the RERTR program goals:

- Little or no chemical interaction is visible between the foil and aluminum, consistent with other FB fuel examinations described in Appendix A. Closer examination with SEM is recommended to confirm that no interaction occurred.

- No bond line is visible between the aluminum layers on any samples at any location, i.e., bonding of aluminum cladding is very effective.

- Bonding of the foil-cladding interface was successfully achieved in two of the three samples:

- Only about $40 \%$ bonding occurred between the foil and aluminum in the sample made with FB tool-face of Steel-A36 (Figure D-1.a and Figure D-1.g in Appendix D show typical bonded and debonded locations). Because no debond is visible in the UT scan (Figure 4.6.a) of the sample made with the steel-faced tool, it is likely that the $60 \%$ debond occurred when the sample was cut from the plate. Debond similarly occurred with other samples later cut from the same plate. Debond of samples from other plates made with a steel-faced tool have also been observed during PIE as noted previously. Such debond occurrence during sample cutting implies a weak bond. This is consistent with previously described bond pull test results, where the sample made with a steel tool had much lower tensile strength than the sample made with an Anviloy tool.

- The sample made with the Al-Bronze tool face has close to $100 \%$ bonding (shown in Figure D-2 in Appendix D).

- The sample made with the Anviloy tool face exhibits about $90 \%$ bonding (shown in Figure D-3 in Appendix D).

The small difference in extent of debond between the latter two samples is likely not significant relative to measurement uncertainties resulting from sample variability and subjectivity of the visual evaluation process.

The extent of foil-cladding bonding in these samples supports the previous theory that the higher heat removal by the Steel-A36 tool leaves less heat in the fuel plate (i.e. a "cold" FB operation), causing lower plate temperatures with less aluminum plasticization than in the other samples. The less-plastic aluminum would result in weaker bonds. Therefore an important conclusion from this study is that aluminum plasticization caused by material movement and elevated temperature during FB is key to determining fuel-cladding bonding success. Tool loads with the FB process deployed here have large and frequent fluctuations, and are one input to drive temperatures. Tool heat extraction efficacy is another determinant of temperature. 


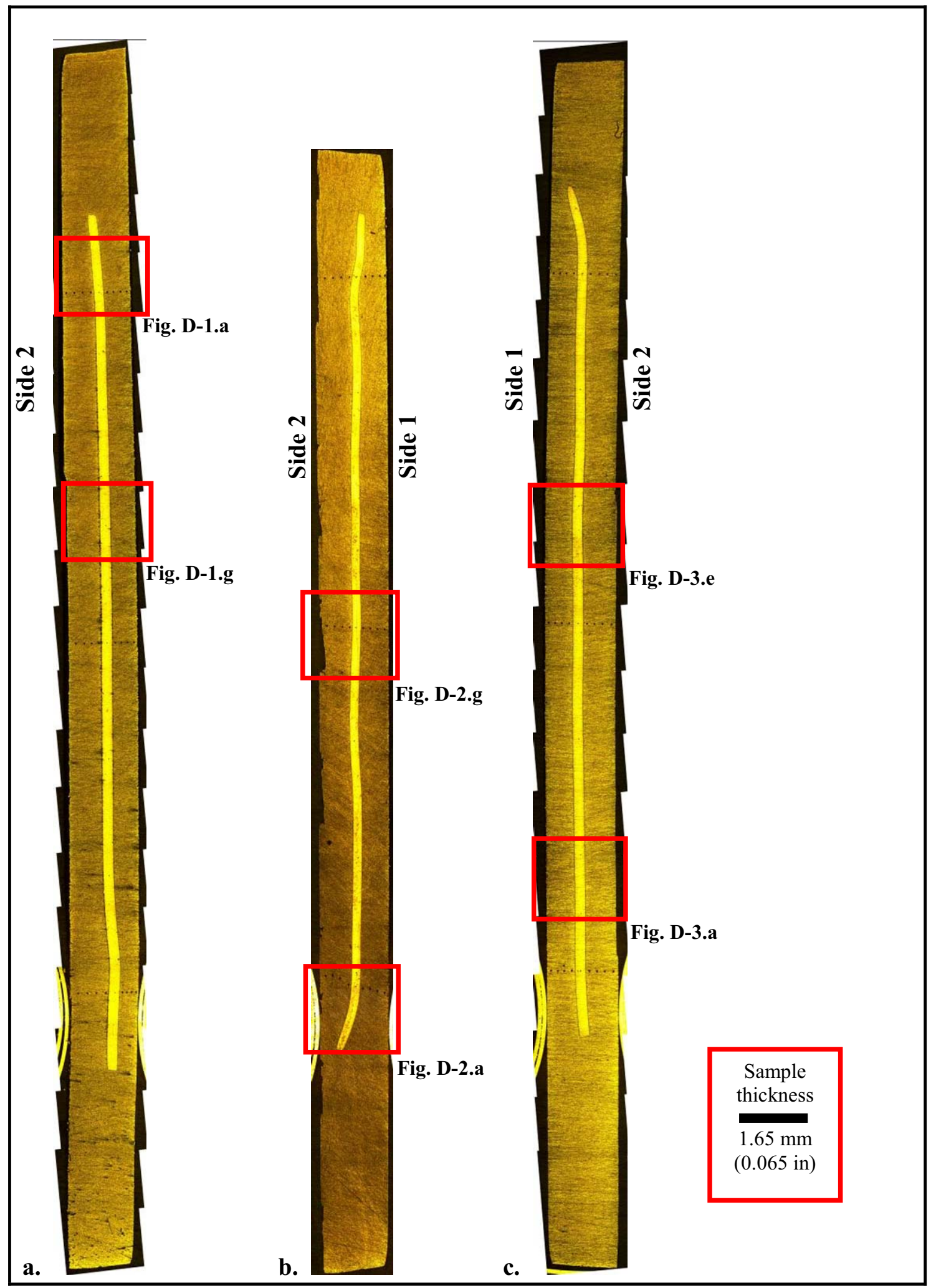

Figure 5.6 Microphotos (50X) stitched together: three cross-sectioned samples (to scale), from surrogate fuel plates fabricated with multiple-pass FB to bond DU10Mo foil inside Al-6061 cladding. Different alloys were used on the FB tool face: a. Steel-A36, b. Aluminum-Bronze, c. Anviloy. Additional photos of the marked locations are available in Appendix D Figures D-1, D-2, and D-3. 
The microscopy examination provides further evidence of aluminum plasticity in the plates during FB. All the foils physically moved location within the cladding during the FB process, especially at the foil edges in the samples made with Al-Bronze and Anviloy FB tools.

Movement of the foil edges was less pronounced in the sample made with the Steel-A36 tool, consistent with the theory of lower aluminum plasticity allowing less foil displacement in this sample. The direction of foil movement (toward either Side 1 or 2) varies among the samples for no apparent reason.

Sample total thickness is consistent with the original total thickness of the two aluminum plates (one with a milled-out pocket) and the foil together, and thickness of the foils is also consistent with pre-FB thickness. However, the two aluminum layers changed in relative thickness along the sample width as the foil was somewhat displaced between them. This is further indication of aluminum plasticity during FB.

Typical Al6061 grain structure (pre-FB) was described previously. After FB, aluminum grains are very fine and distorted throughout all samples, with no differences apparent between samples or between locations within the same sample. Few grains are visible at the optical levels used during these examinations. This level of distortion and fine-sized grains, typical of FSW weld arm and TMAZ microstructure, indicates that the FB plastic strain deformed the grains significantly with little subsequent grain growth.

The grain microstructure observed in the uranium foil was quite different from that observed in the aluminum cladding. Grains in the DU10Mo foil are elongated throughout all samples, with no differences apparent between samples. Elongation occurs in the same direction as the foil width, with a width-to-thickness aspect ratio of about 5:1. These grains appear similar to those in microphotos of other U10Mo pre-FB foils (shown in Figure 2.5), made with a rolling and annealing process comparable to that used to make these foils. (Clark, Hallinan, et al. May 2006) commented that this grain shape and direction is typical for the rolled foil production, and observed that one of those foils underwent FB and retained its asrolled appearance despite the FB. Assuming the samples' foils originally appeared similar to those in Figure 2.5, then similar results, i.e. no change in rolled foil grain appearance despite FB, are observed in this study.

The similar grain appearance in all three samples signifies that no correlation is evident between the FB tool alloy and the fuel plates' grain microstructure.

\section{b. Sample Average Hardness}

Table 5.4 reports sample hardness measurements, which are illustrated in Figure 5.7 and Figure 5.8. Analysis of the hardness data for the three samples yields the following observations.

Average overall aluminum hardness measurements range from $73 \mathrm{H}_{\mathrm{V}}$ to $80 \mathrm{H}_{\mathrm{V}}$, about $28 \%$ less than the typical Al-6061-T6 hardness of $107 \mathrm{H}_{\mathrm{V}}$. This decrease is consistent with hardness reduction that typically occurs due to dynamic recrystallization and dissolution of precipitates during FSW of A16061. The idea of precipitate dissolution is further supported by the fact that these hardness values are comparable to standard hardness of Al6061-T4 [Hv = 75 per (ASM 1990)], which is naturally aged and so has fewer precipitates than pre-FB Al6061-T6, which is tempered at higher than room temperature. The samples' hardness was measured about seven months after the FB was performed, so natural aging had likely occurred and hardness values were higher than they would have been if measured shortly after the FB was performed. 
Table 5.4 Vickers Hardness (HV) measurements across samples from DU10Mo monolithic plates fabricated with differing FB tool face alloys.

\begin{tabular}{|c|c|c|c|c|c|c|c|c|c|c|c|c|c|}
\hline \multirow{4}{*}{$\begin{array}{c}\text { FB Tool } \\
\text { Face Alloy }\end{array}$} & \multirow{4}{*}{$\begin{array}{l}\text { Location } \\
\text { on } \\
\text { Sample }\end{array}$} & \multicolumn{12}{|c|}{ Vickers Hardness $\left(\mathrm{H}_{\mathrm{V}}\right)$} \\
\hline & & \multicolumn{9}{|c|}{ from Side 1 to Side 2} & \multicolumn{3}{|c|}{ Aluminum } \\
\hline & & \multirow[b]{2}{*}{1} & \multicolumn{3}{|c|}{ Aluminum } & \multirow{2}{*}{$\begin{array}{l}\text { Foil } \\
5\end{array}$} & \multicolumn{4}{|c|}{ Aluminum } & \multirow{2}{*}{$\begin{array}{l}\text { Avg. on } \\
\text { Side } 1\end{array}$} & \multirow{2}{*}{$\begin{array}{l}\text { Avg. on } \\
\text { Side } 2\end{array}$} & \multirow{2}{*}{$\begin{array}{c}\text { Overall } \\
\text { Al Avg. } \\
\left(\& \text { StDev }^{\mathbf{b}}\right)\end{array}$} \\
\hline & & & 2 & 3 & 4 & & 6 & 7 & 8 & 9 & & & \\
\hline \multirow[t]{3}{*}{ Steel-A36 } & Top & 71 & 74 & 76 & 75 & $\begin{array}{l}289 \\
276 \\
286\end{array}$ & 73 & 75 & 81 & 83 & 74.0 & 78.0 & $76.0(5.7)$ \\
\hline & Center & 75 & 71 & 72 & 72 & $\begin{array}{l}292 \\
286 \\
284\end{array}$ & 75 & 80 & 78 & 82 & 72.5 & 78.8 & $75.6(4.3)$ \\
\hline & Clip end & 68 & 74 & 80 & 71 & 295 & 77 & 81 & 80 & 83 & 73.2 & 80.2 & $76.8(4.3)$ \\
\hline \multirow[t]{3}{*}{ Al-Bronze } & & 60 & 77 & 74 & 77 & $\begin{array}{l}282 \\
286 \\
288\end{array}$ & 80 & 75 & 81 & 83 & 72.0 & 79.8 & $\begin{array}{l}75.9(7.6) \\
76.4(2.9)\end{array}$ \\
\hline & Center & 74 & 76 & 73 & 73 & $\begin{array}{l}286 \\
264 \\
262\end{array}$ & 79 & 78 & 79 & 79 & 74.0 & 78.8 & $76.6(3.8)$ \\
\hline & Clip end ${ }^{\mathrm{a}}$ & 74 & 70 & 74 & 80 & 258 & 78 & 77 & 80 & 80 & 74.5 & 78.8 & \\
\hline \multirow[t]{3}{*}{ Anviloy } & Top & 77 & 77 & 80 & 80 & $\begin{array}{l}290 \\
290\end{array}$ & 78 & 80 & 84 & 80 & 78.5 & 80.5 & $79.5(2.5)$ \\
\hline & Center & 73 & 73 & 77 & 75 & $\begin{array}{l}288 \\
284 \\
282\end{array}$ & 78 & 78 & 80 & 84 & 74.5 & 80.0 & $77.2(4.0)$ \\
\hline & Clip end & 75 & 75 & 73 & 76 & 279 & 73 & 71 & 74 & 71 & 74.8 & 72.2 & $73.5(2.1)$ \\
\hline \multicolumn{14}{|c|}{$\begin{array}{l}\text { a. All hardness indentations at the Al-Bronze clip-end location had "tails" on them (see Figure D-2.a in Appendix D), due to } \\
\text { an uneven surface on the opposite side of the sample ring. The surface was later smoothed by manual polishing and five } \\
\text { more hardness indentations were taken with results very consistent with the first set reported here. }\end{array}$} \\
\hline
\end{tabular}

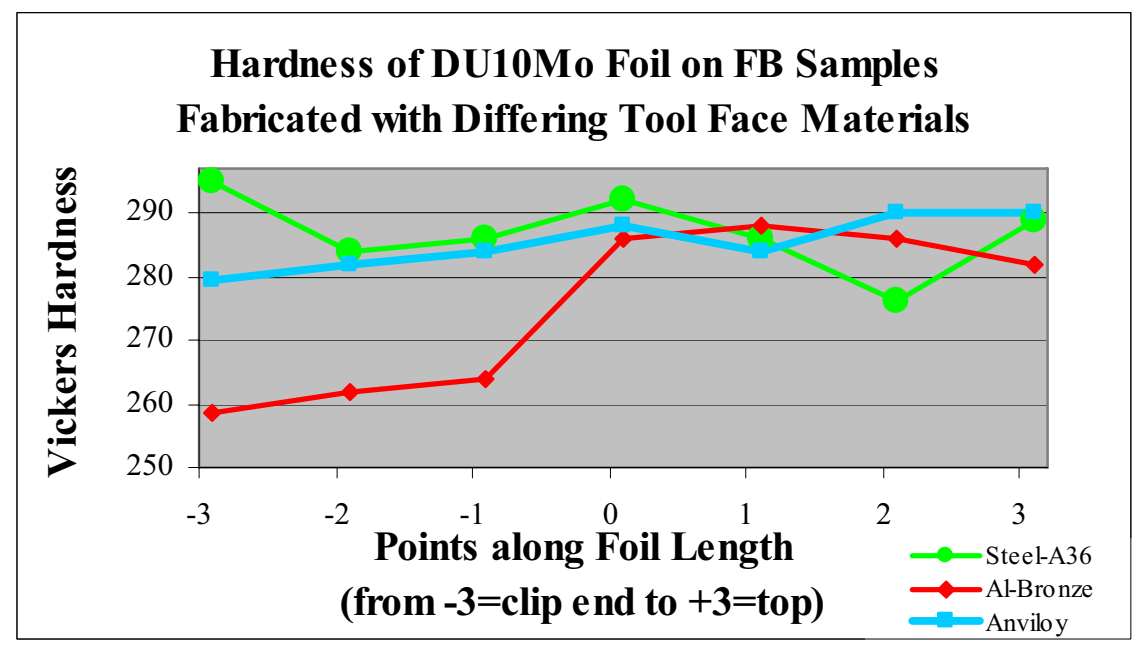

Figure 5.7 Hardness measurements on foil surfaces of three cross-sectioned samples taken from surrogate fuel plates fabricated with multiple-pass FB to bond DU10Mo foil inside aluminum Al-6061 cladding. A different alloy was used on the FB tool face to fabricate each sample. 


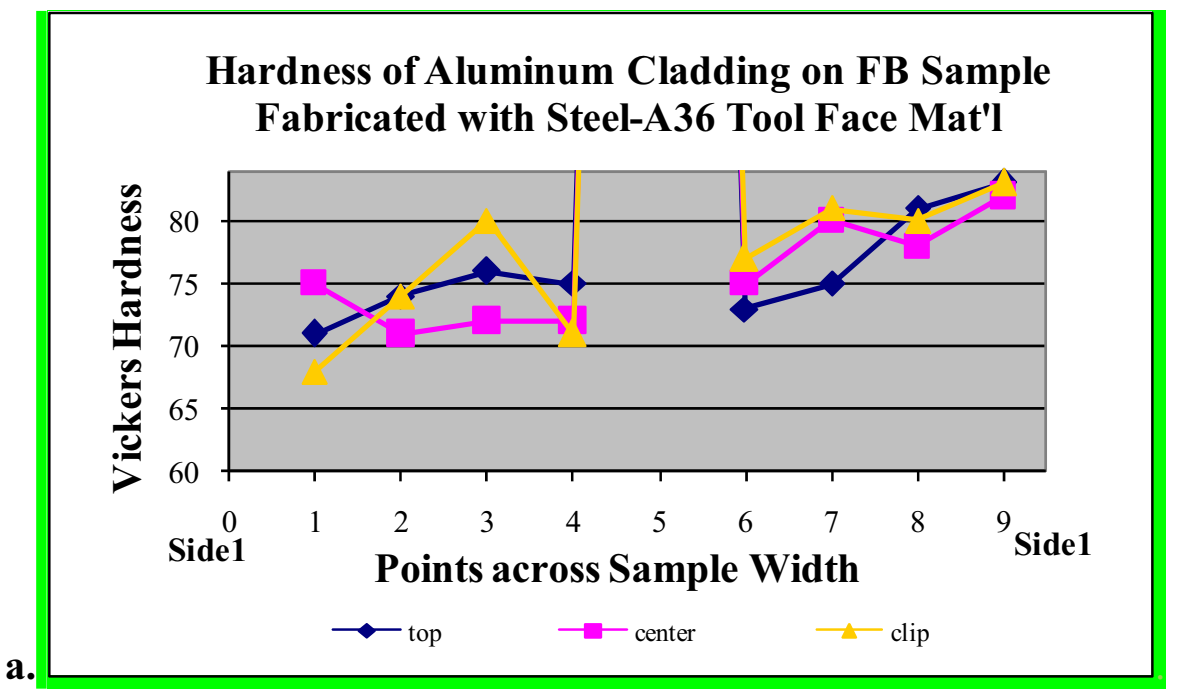

b.

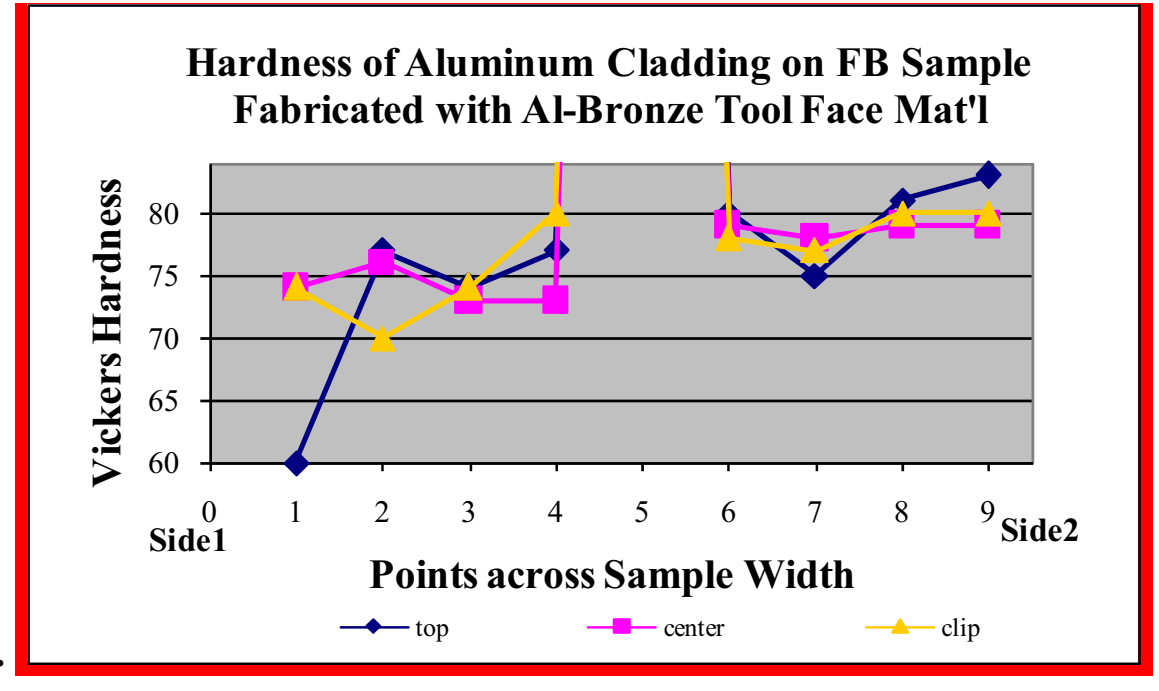

\section{Hardness of Aluminum Cladding on FB Sample} Fabricated with Anviloy Tool Face Mat'l

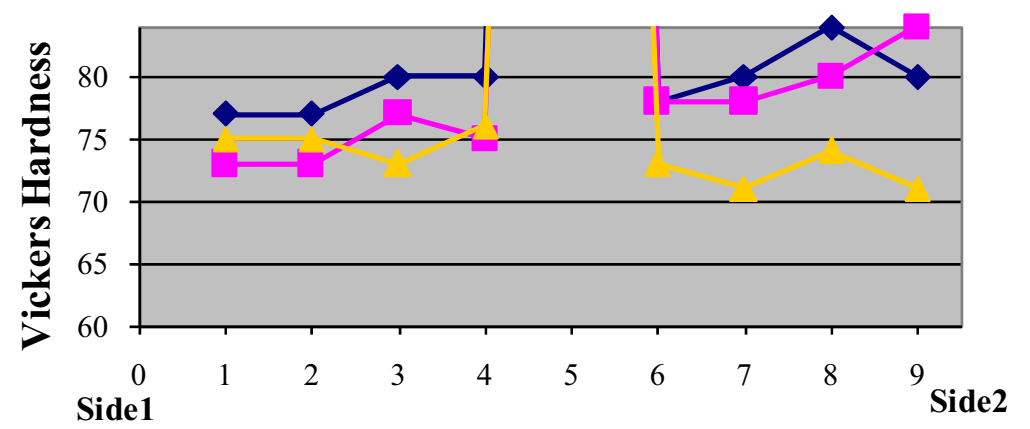

Points across Sample Width

c.

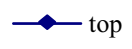

$\rightarrow$ center $\longrightarrow-\operatorname{clip}$

Figure 5.8 Hardness measurements on aluminum surfaces of three cross-sectioned samples taken from surrogate fuel plates fabricated with multiple-pass FB to bond DU10Mo foil inside aluminum Al-6061 cladding. Different alloys were used on the FB tool face: a. Steel-A36, b. Al-Bronze, c. Anviloy. 
It is difficult to quantify the foils' hardness change due to FB, as their original hardness is unknown. No hardness numbers are available for U10Mo foils produced in the same way as these sample foils were. In addition, any hardness numbers available for typical U10Mo foils prior to FB are questionable, because the resistance annealing process is highly variable, based on subjective visual observation using an optical pyrometer. For example, two pre-FB foils, shown in Figure 2.5 and produced using the "initial" rolling and annealing process described previously, had hardness of $270 \pm 6 \mathrm{H}_{\mathrm{V}}$ and $248 \pm 6 \mathrm{H}_{\mathrm{V}}$ (Jue 2006); these are quite different from each other, but both less than most foil hardness measurements listed in Table 5.4. If the foil hardness in these samples had changed from its original hardness, the cause is unknown. Further research would be needed to (1) measure hardness of a foil both before and after FB to learn whether hardness changes due to FB, and (2) if the hardness has changed from the original, perform TEM or SEM examination to gain insight to the change mechanism.

No significant differences are apparent in overall average hardness values between the three samples. Therefore no correlation is apparent between hardness and the tool face alloy. This could be due to the wide variability within the hardness data, even within any one sample: such variability is especially apparent in the Al-Bronze sample. This is discussed later.

\section{c. Location-Specific Tool Temperatures and Sample Hardness}

FB tool load and temperature data measured at sample-specific locations during fabrication of the three fuel plate sets are listed in Table 5.5. Of all data presented in this study, these have the greatest uncertainty due to possible errors in identifying (1) the loads and temperatures associated with the particular location of the sample cross-section within the fuel plate set, and (2) the correct FB pass number associated with the location (top, center, or clip) within the sample. The second procedure was especially difficult on Side 1, where indications of the FB passes had been smoothed out and were hard to detect. These errors and the resulting uncertainty are impossible to quantify for these one-sample evaluations on each plate. Given the uncertainty in the data, it is nevertheless apparent that location-specific temperature trends are consistent with general trends noted previously for the various tool alloys, i.e., the steelfaced tool's internal temperatures were generally lowest of the three tool face alloys and the Anviloy tool's temperatures were highest.

In addition to location uncertainty, there are limitations inherent in using tool internal temperature data to gain insight to the FB process. Due to heat transfer properties of a tool alloy and the coolant, as well as other heat transfer mechanisms during friction bonding, tool internal temperature is not a direct indicator of the absolute value of the plate temperature. Heat extraction efficiency differs depending on the tool alloy, so tool internal temperature during friction bonding with a tool of one alloy is not directly comparable with temperature during friction bonding with a tool of a different alloy. However, tool internal temperatures do indicate the plate temperature in relation to (i.e., higher or lower than) other locations in the same plate or sample. Given this limited use, the sample location-specific temperature data can be compared with other sample location-specific characteristics to see if there are any correlations or trends.

Location-specific tool load and temperature data in Table 5.5 were thus considered. No correlation is possible with grain microstructure, which appears fairly uniform in all locations and in all samples. No correlation is apparent between load data and any other variable, probably because there were large and frequent fluctuations in load across each pass, including in the vicinity of sample locations. Tool internal temperatures were more consistent than tool 
load and it appears there is some correlation between the location-specific temperatures and hardness data. Evaluation of data ${ }^{18}$ in Table 5.4 and Table 5.5 indicates that:

- Both tool internal temperatures and aluminum hardness were generally higher on Side 2 of the samples.

- In general, the higher the tool temperature, the harder the aluminum on Side 2. This pattern was not evident in data for Side 1 of the aluminum.

- Foil hardness was consistently higher on the end of the foil with the highest tool temperatures. For example, for the sample made with FB tool face of Steel-A36, the highest tool temperatures and hardest foil both occurred at the sample's clip end.

A higher tool temperature during FB of the same plate implies that the coolant could not extract the heat fast enough and so the plate temperatures would also be higher at that location. The occurrence of harder aluminum associated with higher temperatures illustrates the effects of tempering (increased temperature causing reforming of hardening precipitates) of grains in A16061. These results are consistent with previous explanation that hardness of A16061 depends strongly on precipitate distribution and only slightly on grain size, and that distribution of the precipitates are significantly affected by temperature (Sato, Kokawa, et al. 2001).

Table 5.5 FB tool load and temperature, measured at specific sample locations during FB of DU10Mo monolithic fuel plates fabricated with differing FB tool face alloys.

\begin{tabular}{|c|c|c|c|c|c|c|c|}
\hline \multirow[b]{2}{*}{$\begin{array}{c}\text { FB Tool } \\
\text { Face Alloy } \\
\& \\
\text { Location } \\
\text { on } \\
\text { Sample }\end{array}$} & \multicolumn{3}{|c|}{ Side 1} & \multicolumn{3}{|c|}{ Side 2} & \multirow[b]{2}{*}{$\begin{array}{c}\text { Average } \\
\text { FB Tool } \\
\text { Temperature } \\
\text { from both } \\
\text { Sides } \\
{ }^{\circ} \mathrm{C}\end{array}$} \\
\hline & \begin{tabular}{|c|} 
Sample's \\
Location in \\
Plate: \\
Percent Distance \\
Across Plate, \\
\& Pass No.
\end{tabular} & $\begin{array}{c}\text { FB } \\
\text { Tool } \\
\text { Load } \\
\text { N } \\
\text { (lbf) }\end{array}$ & $\begin{array}{c}\text { FB Tool } \\
\text { Temperature } \\
{ }^{\circ} \mathrm{C}\end{array}$ & $\begin{array}{c}\text { Sample's } \\
\text { Location in } \\
\text { Plate: } \\
\text { Percent Distance } \\
\text { Across Plate, } \\
\text { \& Pass No. }\end{array}$ & $\begin{array}{c}\text { FB } \\
\text { Tool } \\
\text { Load } \\
\text { N } \\
\text { (lbf) }\end{array}$ & \begin{tabular}{|c} 
FB Tool \\
Temperature \\
${ }^{\circ} \mathrm{C}$
\end{tabular} & \\
\hline $\begin{array}{l}\text { Steel-A36 } \\
\text { Top }\end{array}$ & $\begin{array}{c}75-78 \% \\
\text { Pass } 5\end{array}$ & $\begin{array}{c}52.0 \mathrm{~K} \\
(11.7 \mathrm{~K})\end{array}$ & 127.1 & $\begin{array}{c}75-78 \% \\
\text { Pass5 }\end{array}$ & $\begin{array}{l}49.0 \mathrm{~K} \\
(11.0 \mathrm{~K})\end{array}$ & 139.1 & 133.1 \\
\hline Center & Pass 6 & $\begin{array}{c}48.0 \mathrm{~K} \\
(10.8 \mathrm{~K})\end{array}$ & 128.0 & Pass 4 & $\begin{array}{l}47.5 \mathrm{~K} \\
(10.7 \mathrm{~K})\end{array}$ & 142.4 & 135.2 \\
\hline Clip & Pass 7 & $\begin{array}{c}49.4 \mathrm{~K} \\
(11.1 \mathrm{~K}) \\
\end{array}$ & 132.2 & Pass 3 & $\begin{array}{c}47.0 \mathrm{~K} \\
(10.6 \mathrm{~K})\end{array}$ & 142.6 & 137.4 \\
\hline $\begin{array}{l}\text { Al-Bronze } \\
\text { Top }\end{array}$ & $\begin{array}{l}26-29 \% \\
\text { Pass } 4\end{array}$ & $\begin{array}{c}45.0 \mathrm{~K} \\
(10.1 \mathrm{~K})\end{array}$ & 132.1 & $\begin{array}{l}26-29 \% \\
\text { Pass } 6\end{array}$ & $\begin{array}{c}47.4 \mathrm{~K} \\
(10.7 \mathrm{~K})\end{array}$ & 143.8 & 137.9 \\
\hline Center & Pass 3 & $\begin{array}{c}41.4 \mathrm{~K} \\
(9.3 \mathrm{~K})\end{array}$ & 131.6 & Pass 7 & $\begin{array}{c}45.3 \mathrm{~K} \\
(10.2 \mathrm{~K})\end{array}$ & 142.9 & 137.3 \\
\hline Clip & Pass 2 & $\begin{array}{c}46.9 \mathrm{~K} \\
(10.5 \mathrm{~K}) \\
\end{array}$ & 130.7 & Pass 8 & $\begin{array}{c}44.7 \mathrm{~K} \\
(10.0 \mathrm{~K}) \\
\end{array}$ & 142.1 & 136.4 \\
\hline $\begin{array}{l}\text { Anviloy } \\
\text { Top }\end{array}$ & $\begin{array}{c}26-28 \% \\
\text { Pass } 4\end{array}$ & $\begin{array}{c}45.7 \mathrm{~K} \\
(10.3 \mathrm{~K})\end{array}$ & 135.2 & $\begin{array}{l}26-28 \% \\
\text { Pass } 6\end{array}$ & $\begin{array}{c}48.3 \mathrm{~K} \\
(10.9 \mathrm{~K})\end{array}$ & 150.9 & 143.0 \\
\hline Center & Pass 3 & $\begin{array}{c}43.8 \mathrm{~K} \\
(9.8 \mathrm{~K})\end{array}$ & 134.0 & Pass 7 & $\begin{array}{l}50.3 \mathrm{~K} \\
(11.3 \mathrm{~K})\end{array}$ & 148.3 & 141.1 \\
\hline Clip & Pass 2 & $\begin{array}{l}43.3 \mathrm{~K} \\
(9.7 \mathrm{~K})\end{array}$ & 129.2 & Pass 8 & $\begin{array}{l}48.3 \mathrm{~K} \\
(10.9 \mathrm{~K})\end{array}$ & 149.9 & 139.6 \\
\hline
\end{tabular}

a. See Figure 4.6.a, b and c for illustration of samples' locations in plates.

\footnotetext{
${ }^{18}$ Identification of these trends does not consider evaluation of variance in the data.
} 
Similarly, the correlation of foil hardness with temperature might also be caused by tempering effects. TEM or SEM examination of the foil would provide more detailed information on morphology, which may reveal the reason for hardness differences.

The reason that Side 1 of the aluminum does not exhibit this pattern is unknown. If in fact the temperature data are accurate and not obscured by error in identifying the location-specific temperatures on Side 1, then perhaps the reason that Side 1 does not show a correlation between hardness and temperature has to do with the generally lower temperatures on Side 1. It can be speculated that since temperatures were lower, the FB induced higher strain and more grain boundary disruption and/or recrystallization with consequent lower hardness. And temperatures might not have been high enough to cause tempering to the same degree as occurred on Side 2.

An additional possible reason that Side 1 does not show a correlation between hardness and temperature might be the very low temperature measurement (next to Side 1) on the Al-Bronze sample's top location. If this point is removed from the data set, then Side 1 of that sample does in fact show better correlation between hardness and temperature.

Another factor that surely impacts heat transfer and therefore temperatures and material strain effects through the plate thickness is the presence of the foil in the center. While the aluminum sample group discussed previously showed FB effects across the entire sample thickness, the uranium foil probably acts as a heat reflector or insulator due to its very different thermal and material properties from aluminum. The function of the foil regarding heat transfer in a plate, and the impact of this on the FB process and resulting plate characteristics, require further investigation.

Given the apparent correlation between location-specific temperatures and hardness, and the average location-specific temperatures and FB tool alloys, it is not clear why no correlation appears to exist between hardness and tool alloys. For example, if the sample fabricated with the steel tool really was at lower plate temperature than the other samples, and locationspecific hardness within the sample seems to correlate with location-specific tool temperature, then why isn't there a difference between the average hardness of this sample and the average hardness of the other samples? This phenomenon might be explained by the wide variability within the hardness data, even within any one sample. Such variability is especially apparent, for example, in the Al-Bronze sample. Another possible explanation is the uncertainty inherent in identifying the particular temperature data that applied to these samples' specific locations and, therefore, the speculative nature of conclusions based on these correlations. If the correlation between hardness and temperature is to be better understood, further investigation or study should include (1) direct measurement of plate temperature during FB operations, and (2) more precise association of temperature data with sample locations in the plate. 


\subsection{CONCLUSIONS AND RECOMMENDATIONS}

The objective of the research presented in this thesis is to provide insight into the FB process and its application to the design of monolithic fuel. Specific emphasis is on recognizing and understanding the microstructural effects of varying two fabrication parameters: (a) FB tool load, and (b) FB tool face alloy. This section compiles and summarizes conclusions and recommendations evolved from the study.

\subsection{Conclusions from this Thesis Study}

\subsubsection{Conclusions from Examination of Aluminum Samples Fabricated with Varying Tool Load}

Conclusions garnered during the previous analysis of aluminum samples made with varying tool load are compiled in this section.

It was concluded that successful aluminum cladding-cladding bonds can be attained in actual fuel plates using any of the tested FB tool loads [35,600 N through 62,300 N (8,000 lbf through 14,000 lbf)]. This assumes other FB operating parameters the same as used in these experiments.

It was concluded that samples produced with larger FB tool loads exhibit characteristics of increased work input and associated higher temperatures and forging: they have a wider FB path, increased bonding, finer grains, and increased hardness.

FB produces significant differences in material properties between advancing and retreating edges and between FB sides and non-FB sides. It was therefore concluded that, if homogeneous material properties are desired in fuel plates, then current FB practices should be continued: i.e., overlapping FB passes and FB performance on both sides of the assembly.

The samples exhibit reduced hardness and grain size, with variation evident between advancing and retreating tool edges and across sample thicknesses. Such sample characteristics are consistent with phenomena observed in FSW/FSP studies and with temperature data gathered during other INL FB studies. It was thus concluded that understanding of FSW/FSP material movement and temperature effects on aluminum translates well to the FB process. Since high quality FSW welds can be obtained for a wide range of fabrication parameter combinations, this bodes well for the robustness of the FB process.

It was concluded that aluminum plasticization during FB is a key driver of material properties and sample characteristics. Thus FB process variables that impact aluminum plasticization via material movement and temperature are important to determining those characteristics. Many such variables exist and by nature their effects are complex and hard to predict.

\subsubsection{Conclusions from Friction Bonding and Examination of DU10Mo Samples Fabricated with Varying Tool Face Alloys}

This section compiles conclusions garnered during the previous analyses of loads and temperatures measured during friction bonding of DU10Mo fuel plates with varying tool face alloys, and examination results on samples subsequently taken from each of those fuel plates. 
It was concluded that, under FB operating parameters used in these experiments, the Anviloy tool face produced the best combination of bonding and aluminum surface finish. Relative performance of FB with the three tool face alloys indicates that:

- The steel tool face has highest heat extraction efficiency, yielding good aluminum surface texture but inadequate U-Al bonding due to low FB plate temperatures.

- The aluminum-bronze tool face has lowest wear-resistance and lowest heat extraction efficiency, yielding good bonding but rough aluminum surface texture due to excess extrusion caused by high FB plate temperatures.

- The Anviloy tool face has intermediate heat extraction efficiency, yielding good bonding and good surface finish, due to intermediate FB plate temperatures.

Regardless of FB tool alloy, plastic strain caused by FB cold working creates uniformly very fine and distorted grains throughout the fuel plate's aluminum cladding, and no apparent change in foil grain appearance. Thus no correlation is evident between the fuel plates' grain microstructure appearance and the FB tool face alloy.

No significant differences are apparent in overall average hardness values between the three samples. Therefore no correlation is apparent between hardness and the FB tool face alloy.

Little or no chemical reaction layer is visible between the uranium foil and aluminum cladding. If confirmed by closer examination with SEM, it can be concluded that under FB operating parameters used in these experiments, little or no such reaction occurs at the foilcladding interface, meeting the RERTR fuel development goal of minimal interface reaction during fabrication.

No bond line is visible between the aluminum layers on any DU10Mo samples at any location. It can therefore be concluded that very effective bonding of aluminum cladding occurs under the FB operating parameters used in these experiments.

Reduced aluminum hardness indicates that recrystallization and/or dissolution of hardening precipitates occurred. The occurrence of harder material in the samples at specific locations where higher FB temperatures also occurred illustrates the effects of tempering in Al6061. Such sample characteristics are consistent with phenomena observed in FSW/FSP studies and with temperature data gathered during other INL FB studies.

It was concluded that at the flow rate used in these experiments, the coolant system did not have the heat removal capacity to keep up with the FB work input, so that during FB operations there was a cumulative increase in internal tool temperatures and therefore plate temperatures.

It was concluded that aluminum plasticization resulting from material movement and elevated temperature during FB is key to determining many material properties and fuel plate characteristics. Such characteristics include cladding-cladding and fuel-cladding bonding success, grain size and hardness, change in relative thickness of aluminum layers and foil position between layers, and the texture of the aluminum surface after FB. Variables driving the material movement and temperature are numerous, and by nature their effects are complex and hard to predict. Two such variables are tool load and tool face alloy. 


\subsubsection{Summary of Conclusions}

This section summarizes the most important conclusions compiled above.

\section{a. Conclusions Regarding Tool Load}

Tool load provides work input to the FB process and thus is a driver of material movement and temperatures that plasticize the aluminum. An increased FB tool load results in a wider FB path, increased bonding by forging, finer grains, and increased hardness. Under FB operating parameters used in these experiments, successful aluminum cladding-cladding bonds can be attained in actual fuel plates using a wide range of FB tool loads. The range of tool loads yielding successful foil-cladding bonding was not established, but it was demonstrated that such bonding can be attained with FB tool load of 48,900 N (11,000 lbf) when using the Anviloy-faced FB tool.

\section{b. Conclusions Regarding Tool Alloys}

Under FB operating parameters used in these experiments, the Anviloy tool face produced an acceptable and better combination of bonding and aluminum surface finish than the SteelA36 tool face or the Al-Bronze tool face. The steel-faced tool did not produce acceptable bonding at the foil-cladding interface. The Al-Bronze tool produced an undesirably rough aluminum surface finish, indicative that FB process temperatures were too high.

No correlation was evident between the FB tool face alloy and resulting grain microstructure appearance or hardness.

\section{c. Conclusions Regarding the Friction Bonding Process}

Results of this study provide support and validation for other RERTR studies and conclusions. For example, FB performed with operating parameters used in these experiments successfully meets some of the RERTR goals for monolithic fuel: successful cladding-cladding bonding; successful foil-cladding bonding; and little or no chemical reaction at the foilcladding interface. This study also demonstrates that if homogeneous material properties are desired in fuel plates, current practices of overlapping FB passes on both sides should be continued. In addition, these study results are consistent with results of other FB studies such as the sample pull tests, the FB tungsten tracer experiment, and the thermal model.

This study demonstrates that aluminum plasticization is a key determinant of fuel plate characteristics such as surface texture, material properties, and bonding between layers. Therefore FB process variables that impact aluminum plasticization via material movement and temperature are important to determining those fuel plate characteristics, although there are many such variables and their interaction is by nature complex and hard to predict. Two such variables are FB tool load and heat extraction by the tool from the FB process: this study shows that increased tool load increases the plasticization and forging, and that the tool face alloy helps determine the tool's heat extraction efficacy.

Many material properties and sample characteristics observed during this study correlate well with results reported for FSW/FSP on aluminum. It is therefore apparent that an understanding of FSW/FSP material movement and temperature effects on aluminum translates well to the FB process. Since high quality FSW welds can be obtained for a wide range of fabrication parameter combinations, this bodes well for the robustness of the FB process. 


\subsection{Recommendations for Future Research}

\subsubsection{Recommendations}

This section compiles recommendations made during the previous discussion of examination results on the aluminum and DU10Mo fuel plate samples, and analyses of loads and temperatures measured during friction bonding of DU10Mo fuel plates.

Little or no chemical reaction layer is visible between the foil and cladding in the DU10Mo samples. Closer examination with SEM is recommended to confirm that no interface chemical reaction occurred.

This study explored the effect of a wide range of FB tool loads on aluminum plates, but did not explore the effect on fuel plates with foils. The latter has been explored via tungsten tracer tests performed by other RERTR studies. Further research via FB of fuel plates with varied tool load and subsequent microstructural examination is recommended to (1) understand the effects of tool load on the foil-cladding bonds, and (2) identify a range of tool load that can be used to produce acceptable monolithic fuel plates.

It is unknown whether foil hardness in these samples changed from its original hardness; if so, the cause is unknown. Further research is recommended to (1) measure hardness of a foil both before and after FB to learn how hardness changes due to FB, and (2) if the hardness has changed from the original, perform TEM and/or SEM examination on both the pre- and postFB samples to help understand the change mechanism.

Further research is recommended to aid understanding of the correlation between temperature and other characteristics of FB fuel plates. Such research should include (1) direct measurement of plate temperature during FB operations, and (2) more precise association of temperature data with specific sample locations in the plate.

The thermal model described by (Dixon, Burkes and Medvedev 2007) should be further developed to better correlate output with empirical measurements of plate temperatures, and represent the foil presence in the fuel plate assembly. Such development could incorporate insight gained from research provided per the previous recommendation.

Research is recommended to understand the foil's function regarding heat transfer and material movement in the fuel plate, and the impact of this on the FB process and resulting fuel plate characteristics. Such research could include aspects of the previous two recommendations.

Further investigation is needed to understand why the first FB pass on each side of these three DU10Mo fuel plates required lower tool load.

The coolant system capacity should be increased to adequately remove heat from the FB tool, so that constant plate and tool temperatures can be maintained during FB when all other variables are also held constant. This will aid future research and evaluation of the impact of varying other variables.

As changes are explored in FB operating parameters or fuel plate materials, similar samples should be produced by FB with all other operating parameters and materials the same as used in these experiments. Examination results from those samples can then be compared with these results to help understand any impact of the changed parameters or materials.

Similar samples will be produced by FB on the new TTI machine at FASB, using the same FB operating parameters and materials as used in these experiments. Examination results from those samples should then be compared with these results to help understand any impact of 
using the new machine, and provide a baseline for evaluation of items produced on the new machine relative to those produced on the current machine.

Pull tests should be performed on some remaining portions of the plates from which samples were taken for these experiments. Pull test results should then be compared with results of the previous pull tests on other samples, and with these examination microstructural examination results, to identify any trends or correlations.

When available, PIE results on the RERTR-9A fuel should be compared with these examination results to identify impacts of irradiation.

\subsubsection{Summary of Recommendations}

This section summarizes the most important recommendations compiled above.

\section{a. Further Examination of These Samples and Fuel Plates}

Further examination of the samples and fuel plates used in this study is relatively easy. Such further research should include SEM examination of the foil-cladding bonds for signs of interface chemical reaction. Also, pull tests should be performed on some remaining portions of these study's plates, with subsequent comparison with other examination results.

\section{b. Research on Friction Bonding Temperatures}

Since temperature during FB is an important driver of material properties and fuel plate characteristics, further research is recommended to better understand the temperature source and effects. Such research should include direct measurement of plate temperature during FB operations, more precise association of temperature data with specific sample locations in the plate, and further development of the existing FB thermal model. To support this research, the coolant system capacity should be increased to adequately remove heat from the FB tool, so that constant plate and tool temperatures can be maintained during FB when all other variables are also held constant.

\section{c. Research on Foil}

Research is recommended on the foil's function regarding heat transfer in the plate, and the impact of this on the FB process and resulting plate characteristics. As part of this, changes in foil hardness should be quantified and explained.

\section{d. Comparison with Future Sample Data}

Data and conclusions from this study should be used to evaluate results from future FB studies. Such studies should include:

1. Fuel plates (with foils) made with varied FB tool load, to identify a range of tool load that can be used to produce acceptable monolithic fuel plates.

2. Fuel plates made with other varied FB operating parameters or fuel plate materials, to understand the effects and acceptability of such variations in producing monolithic fuel plates.

3. Fuel plates made by FB on the new TTI machine at FASB using the same FB operating parameters and materials as used in these experiments. This will aid to understanding impacts of FB with the two different machines.

4. PIE of RERTR-9a fuel to recognize effects of irradiation. 


\subsection{REFERENCES}

Adams-Hughes, M., P.N. Kalu, M.K. Khraisheh, and N. Chandra. "Microcharacterization and texture analysis of friction stir processed AA 5052 alloy." Friction Stir Welding and Processing III. San Francisco, CA: TMS, 2005. 3-10.

Arbegast, William J. "Friction stir welding: After a decade of development." Friction Stir Welding and Processing IV. Orlando, FL: TMS, 2007. 3-18.

Arbegast, William J. "Modeling friction stir joining as a metalworking process." Edited by Z. Jins. Hot Deformation of Aluminum Alloys III. TMS, 2003. 313-327.

Arbegast, William J. "Using process forces as a statistical process control tool for friction stir welds." Friction Stir Welding and Processing III. San Francisco, CA: TMS, 2005. 193204.

ASM Handbook Vol. 2, 1990. "Properties and Selection: Nonferrous Alloys and SpecialPurpose Materials." ASM Int'1, 10th Ed. (Handbook indicates Brinell Hardness $(\mathrm{HB})=$ 95 for Al-6061-T6. HB value was converted to $107 \mathrm{Hv}$ value on August 20, 2007, by Matweb Material Property Data website at: http://www.matweb.com/search/SpecificMaterial.asp?bassnum=MA6061T6.), 1990.

ASTM C633-01 (2006a). "Standard test method for adhesion or cohesion strength of thermal spray coatings. "West Conshohocken, USA: ASTM, 2006a.

ASTM E112-96 (2004). "Standard test methods for determining average grain size." West Conshohocken, USA: ASTM, 2004.

ASTM E384-06 (2006b). "Standard test method for microindentation hardness of materials." West Conshohocken, USA: ASTM, $2006 \mathrm{~b}$.

AWS, Subcommittee D17J. "Specification for Friction Stir Welding of Aluminum Alloys for Aerospace Applications (Draft)." No. D17.3:200X.

Benavides, S., Y. Li, L.E. Murr, D. Brown, and J.C. McClure. "Low-temperature friction-stir welding of 2024 aluminum." Scripta Materialia (Elsevier Science Ltd., Acta Metallurgica Inc.) Vol. 41, no. 8 (1999): 809-815.

Bhanumurthy, Karanam, Nitin Kumbhar, and Beant Prakash Sharma. "Effect of heat treatment on microstructure, mechanical properties and composition variation across the interface for the FSW $6061 \mathrm{Al}$ alloy weldments." Friction Stir Welding and Processing IV. Orlando, FL: TMS, 2007. 379-388.

Burkes, Douglas E. Verbal communication during Jan.-Mar. 2008.

Burkes, Douglas E., Dennis D. Keiser, Daniel M. Wachs, J.S. Larson, and Michael D. Chapple. "Characterization of monolithic fuel foil properties and bond strength." International Topical Meeting on Research Reactor Fuel Management (RRFM) - 2007. Lyon, France: Idaho National Laboratory INL/CON-07-12208, March 2007.

Burkes, Douglas E., Dennis D. Keiser, Greg K. Miller, Daniel M. Wachs, J.S. Larson, and Francine J Rice. "Update on bonding in monolithic fuel." RERTR Independent Program Review meeting. Unpublished presentation given at the Idaho National Laboratory, January 2007a.

Burkes, Douglas E., N. Patrick Hallinan, Curtis R. Clark, Glenn A. Moore, and Michael D. Chapple. "Update on friction stir welding." RERTR Independent Program Review 
meeting. Unpublished presentation given at the Idaho National Laboratory, January $2007 b$.

Burkes, Douglas E., N. Patrick Hallinan, Jared M. Wight, and Michael D. Chapple. "Update on friction bonding of monolithic U-Mo fuel plates." International Meeting on Reduced Enrichment for Research and Test Reactors (RERTR) - 2007. Prague, Czech Republic, September 2007.

Burkes, Douglas E., N. Patrick Hallinan, Karen L. Shropshire, and Peter Wells. "Effects of applied load on 6061-T6 aluminum joined employing a novel friction bonding process." Under review for publication in Metallurgical and Materials Transactions A (TMS), 2008.

Burkes, Douglas E., N. Patrick Hallinan, Michael D. Chapple, and Karen L. Shropshire. "Friction Bonding." RERTR Independent Program Review meeting. Unpublished presentation given at the Idaho National Laboratory, January 2008.

Cai, B., D. Huang, B.L. Adams, and T.W. Nelson. "Microstructure characteristics of friction stir processed Al 7075 via macroscopic approach." Friction Stir Welding and Processing III. San Francisco, CA: TMS, 2005. 269-275.

Chen, Yen-Lung. "Microstructures and mechanical properties of friction stir welded AA5754O and AA5182-O aluminum tailor-welded blanks." Friction Stir Welding and Processing IV. Orlando, FL: TMS, 2007. 143-154.

Clark, Curtis R., and R.J. Briggs. "Development of a monolithic research reactor fuel type at Argonne National Laboratory." Americas Nuclear Energy Symposium. Miami, FL, 2004.

Clark, Curtis R., et al. "Update on U.S. high density fuel fabrication development." International Topical Meeting on Research Reactor Fuel Management (RRFM) - 2007. Lyon, France: Idaho National Laboratory INL/CON-07-12355, 2007.

Clark, Curtis R., Jan-Fong Jue, Glenn A. Moore, N. Patrick Hallinan, and Blair H. Park. "Update on monolithic fuel fabrication methods." International Meeting on Reduced Enrichment for Research and Test Reactors (RERTR) - 2006. Cape Town, South Africa: Idaho National Laboratory INL/CON-06-11897, October 2006.

Clark, Curtis R., Mitchell K. Meyer, and Steven L. Hayes. "RERTR fuel development at Argonne National Laboratory." Americas Nuclear Energy Symposium. Miami, FL, 2002.

Clark, Curtis R., N. Patrick Hallinan, Jan-Fong Jue, Dennis D. Keiser, and Jared M. Wight. "Monolithic fuel fabrication process development." International Topical Meeting on Research Reactor Fuel Management (RRFM) - 2007. Sofia, Bulgaria: Idaho National Laboratory INL/CON-06-01370, May 2006.

Clark, Curtis, R., G.C. Knighton, Mitchell K. Meyer, and G.L. Hofman. "Monolithic fuel plate development at Argonne National Laboratory." International Meeting on Reduced Enrichment for Research and Test Reactors (RERTR) - 2003. Chicago, IL, 2003.

Colligan, Kevin J. "Relationships between process variables related to heat generation in friction stir welding of aluminum." Friction Stir Welding and Processing IV. Orlando, FL: TMS, 2007. 39-54.

DeMint, Amy, Randy J. Dunavant, Jack Gooch, and Trent C. Andes. "Update on uraniummolybdenum fuel foil fabrication development activities at the Y-12 National Security 
Complex in 2007." International Meeting on Reduced Enrichment for Research and Test Reactors (RERTR) - 2007. Prague, Czech Republic, 2007.

Dixon, John, Douglas Burkes, and Pavel Medvedev. "Thermal modeling of a friction bonding process." COMSOL Conference. Boston, MA. October, 2007.

DOE. U.S. Department of Energy DOE-HDBK-1017/1-93, Fundamentals Handbook, Materials Science. Vol. 1 of 2, pg. 13. Washington, D.C.: OSTI, Oak Ridge, TN, 1993.

Finlay, M. Ross, Daniel M. Wachs, Adam Robinson, and G.L. Hofman. "Post irradiation examination (PIE) of monolithic mini-fuel plates from RERTR-6 \& 7." International Topical Meeting on Research Reactor Fuel Management (RRFM) - 2007. Lyon, France, 2007.

Finlay, M. Ross, Daniel M. Wachs, and G.L. Hofman. "Post irradiation examination (PIE) of monolithic mini fuel plates from RERTR-6." International Meeting on Reduced Enrichment for Research and Test Reactors (RERTR) - Oct. 2006. Cape Town, South Africa. October, 2006.

Flinn, Richard A., and Paul K. Trojan. Engineering Materials and Their Applications (textbook). Boston: Houghton Mifflin Co., 1975.

Fonda, R.W., J.F. Bingert, and K.J. Colligan. "Development of grain structure during friction stir welding." Scripta Materialia (Elsevier Ltd., Acta Materialia Inc.) Vol. 51 (2004): 243-248.

GordonEngland, website:. http://www.gordonengland.co.uk/hardness. Viewed on February 8, 2008.

Gould, Jerry E., and Zhili Feng. "Heat flow model for friction stir welding of aluminum alloys." Journal of Materials Processing \& Manufacturing Science (Technomic Publishing Co., Inc.) Vol. 7 (October 1998): 185-194.

Hultren, R. Fundamentals of Physical Metallurgy, pg. 158. 1952.

Huneau, Bertrand, Xavier Sauvage, Surendar Marya, and Arnaud Poitou. "Microstructure evolution during friction stir welding of commercial aluminum alloys." Friction Stir Welding and Processing III. San Francisco, CA: TMS, 2005. 253-260.

Jarousse, C., P. Lemoine, P. Boulcourt, W. Petry, and A. Rohrmoser. "Last manufacturing results of monolithic UMo full size prototype plates." International Meeting on Reduced Enrichment for Research and Test Reactors (RERTR) - 2006. Cape Town, South Africa, 2006.

Jarousse, C., P. Lemoine, P. Boulcourt, W. Petry, and A. Rohrmoser. "Monolithic UMo full size prototype plates for IRIS V irradation." International Topical Meeting on Research Reactor Fuel Management (RRFM) - 2007. Lyon, France, 2007.

Jin, H., S. Saimoto, M. Ball, and P.L. Threadgill. "Characterization of microstructure and texture in friction stir welded joints of 5754 and 5182 aluminum alloy sheets." Materials Science and Technology (Publisher: Maney Publishing on behalf of the Institute of Materials, Minerals and Mining) Vol. 17 (December 2001): 1605-1614.

Jue, Jan-Fong. "Summary of RERTR microstructure and hardness as of 03-06." Unpublished work by INL RERTR program, 2006. 
Jue, Jan-Fong, Curtis R. Clark, and Michael Nilles. "Hot isostatic pressing (HIP)." RERTR Independent Program Review. Unpublished presentation given at the Idaho National Laboratory, 2008.

Kalya, P., K. Krishnamurthy, Rajiv Mishra, and John Baumann. "Specific energy and temperature mechanistic models for friction stir processing of Al-F357." Friction Stir Welding and Processing IV. Orlando, FL: TMS, 2007. 113-126.

Keiser, Dennis D, Adam B. Robinson, and M. Ross Finlay. "Observations derived from the characterization of monolithic fuel plates irradiated as part of the RERTR-6 experiment." International Meeting on Reduced Enrichment for Research and Test Reactors (RERTR) - 2007. Prague, Czech Republic, 2007.

Keiser, Dennis D, Jan-Fong Jue, and Douglas E. Burkes. "Characterization and testing of monolithic RERTR fuel plates." International Topical Meeting on Research Reactor Fuel Management (RRFM) - 2007. Lyon, France: Idaho National Laboratory INL/CON-07-12224, 2007.

Keiser, Dennis D., Jan-Fong Jue, and Curtis R. Clark. "UMo foil/cladding interactions in friction stir welded monolithic RERTR fuel plates." International Meeting on Reduced Enrichment for Research and Test Reactors (RERTR) - 2006. Cape Town, South Africa: Idaho National Laboratory INL/CON-06-11914, 2006.

Kim, Yeon Soo, G.L. Hofman, P.G. Medvedev, G.V. Shevlyakov, A.B. Robinson, and H.J. Ryu. "Post irradiation analysis and performance modeling of dispersion and monolithic U-Mo fuels." International Meeting on Reduced Enrichment for Research and Test Reactors (RERTR) - 2007. Prague, Czech Republic, 2007.

Krishnan, K.N. "On the formation of onion rings in friction stir welds." Materials Science \& Engineering (Elsevier Science Ltd) Vol. A327 (2002): 246-251.

LECO Hardness Tester. ConfiDent Software Version 2.5.1 by LECO (c)1998-2002, in conjunction with a Dell PC and a LECO Corp. Model LM-100 hardness tester and optical microscope. (Set at default conditions of 640x480 source, 50X objective, and $100 \mathrm{gf}$ load for Vickers indentor), 2002.

Liu, A.G., L.E. Murr, C-S. Niou, J.C. McClure, and F.R. Vega. "Microstructural aspects of the friction-stir welding of 6061-T6 aluminum." Scripta Materialia (Elsevier Science LTD, Acta Metallurgica Inc.) Vol. 37, no. 3 (1997): 355-361.

Liu, L., H. Nakayama, S. Fukumoto, A. Yamamoto, and H. Tsubakino. "Microstructure of friction stir welded 6061 aluminum alloy." Edited by J.F. Nie, A.J. Morton and B.C. Muddle. Materials Forum (Institute of Materials Engineering Australasia Ltd.) Vol. 28 (2004): 878-882.

Lumsden, Jesse, Gary Pollock, and Murray Mahoney. "Effect of tool design on stress corrosion resistance of FSW AA7050-T7451." Friction Stir Welding and Processing III. San Francisco, CA: TMS, 2005. 19-26.

Mahoney, M.W., C.B. Fuller, M.P. Miles, and W.H. Bingel. "Thick plate bending of friction stir processed aluminum alloys." Friction Stir Welding and Processing III. San Francisco, CA: TMS, 2005. 131-138.

Marzoli, L.M., R. Zettler, J.F. dos Santos, M. Volpone, and E. Rizzuto. "A preliminary investigation on the microstructure and properties of friction stir welds in an 
A17075/A12O3/10\% reinforced alloy." Friction Stir Welding and Processing III. San Francisco, CA: TMS, 2005. 243-252.

Material properties database, sponsored by K\&K Associates TAK 2000 software - Website: http://www.tak2000.com/data/prop1.htm\#Moly. Viewed on December 19, 2007.

Matweb, Materials Property Website:. http://www.matweb.com/search/PropertySearch.aspx. Viewed on December 19, 2007.

McNelley, Terry, et al. "Stir zone temperatures during friction stir processing." Friction Stir Welding and Processing IV. Orlando, FL: TMS, 2007. 403-408.

Mishra, R.S., and Z.Y. Ma. "Friction stir welding and processing." Materials Science and Engineering Reports: A Review Journal (Elsevier Science Ltd.) 50 (August 2005): 178.

Murr, L.E., Ying Li, R.D. Flores, and Elizabeth A. Trillo. "Intercalation vortices and related microstructural features in the friction-stir welding of dissimilar metals." Materials Research Innovations (Springer-Verlag) Vol. 2 (1998): 150-163.

Nelson, Tracy W. "Friction stir welding - a brief review and perspective for the future." Friction Stir Welding and Processing III. San Francisco: TMS, 2005. 149-160.

Oberembt, Clark, Casey Allen, William Arbegast, and Anil Patnaik. "Screening for process variable sensitivity in refill spot welding of 6061 aluminum sheet." Friction Stir Welding and Processing IV. Orlando, FL: TMS, 2007. 379-388.

Olympus IX70 optical microscope. Used in conjunction with a Dell PC and "PAXit!" software, Version 6.6.

Pao, P.S., R.W. Fonda, H.N. Jones, C.R. Feng, B.J. Connolly, and A.J. Davenport. "Microstructure, fatigue crack growth, and corrosion in friction stir welded Al 5456." Friction Stir Welding and Processing III. San Francisco, CA: TMS, 2005. 27-34.

Querin, Joseph, A.M. Davis, and Judy A. Schneider. "Effect of processing parameters on microstructure of the FSW nugget." Friction Stir Welding and Processing IV. Orlando, FL: TMS, 2007. 185-192.

Robinson, Adam B., and M. Ross Finlay. RERTR-7 Post Irradiation Examination (PIE). External Report, Idaho National Laboratory INL/EXT-07-13271. September, 2007.

Sato, Yutaka S., Hiroyuki Kokawa, Keiske Ikeda, Masatoshi Enomoto, Shigetoshi Jogan, and Takenori Hashimoto. "Microtexture in the friction-stir weld of an aluminum alloy." Metallurgical and Materials Transactions A (Elsevier Science Ltd) Vol. 32A (April 2001): 941-948.

Sato, Yutaka S., Mitsunori Urata, and Hiroyuki Kokawa. "Parameters controlling microstructure and hardness during friction-stir welding of precipitation-hardenable aluminum alloy 6063." Metallurgical and Materials Transactions A (Elsevier Science Ltd) Vol. 33A (March 2002): 625-635.

Schneider, Judy. Thermo-mechanical processing in friction stir welds. NASA Faculty Fellowship Program, Marshall Space Flight Center and University of Alabama, 2002.

St-Georges, Lyne, Veronique Dassylva-Raymond, Laszlo Kiss, and Alexandre Perron. "Transport phenomena in friction stir welding." Friction Stir Welding and Processing $I V$. Orlando, FL: TMS, 2007. 89-100. 
Swank, W. David, DC Haggard, and Douglas E. Burkes. "Plasma application of silicon." RERTR Independent Program Review. Unpublished presentation given at the Idaho National Laboratory - January, 2008.

Thomas, W.M., Edward D. Nicholas, James C. Needham, Michael G. Murch, Peter TempleSmith, and Christopher J. Dawes. Friction Stir Butt Welding. UK Patent 9125978.8. December 1991.

TWI, FSW website:. http://www.twi.co.uk/j32k/unprotected/band_1/fswintro.html. Viewed on September 22, 2007.

Vugrin, Tamara, Martin Schmucker, and Gunter Staniek. "Root flaws of friction stir welds - an electron microscopy study." Friction Stir Welding and Processing III. San Francisco, CA: TMS, 2005. 277-284.

Wachs, Daniel M. RERTR Fuel Development and Qualification Plan. External Report, Idaho National Laboratory INL/EXT-05-01017 Rev. 3, April 2007.

Wachs, Daniel M., Dennis D Keiser, Curtis R. Clark, and Douglas E. Burkes. "Overview of fuel development plan." RERTR Independent Program Review. Unpublished presentation given at the Idaho National Laboratory, 2008.

Wachs, Daniel M., Douglas E. Burkes, Steven L. Hayes, and W. Skerjanc. "Modeling the integrated performance of dispersion and monolithic U-Mo based fuels." International Meeting on Reduced Enrichment for Research and Test Reactors (RERTR) - 2006. Cape Town, South Africa, Oct 2006b.

Wachs, Daniel M., et al. "Progress in the development of LEU fuel." Global 2007 Conference Advanced Nuclear Fuel Cycles and Systems. Boise, ID, Sept 2007.

Wachs, Daniel M., Richard G. Ambrosek, Gray S. Chang, and Mitchell K. Meyer. "Design and status of RERTR irradiation tests in the advanced test reactor (ATR)." International Meeting on Reduced Enrichment for Research and Test Reactors (RERTR) - 2006. Cape Town, South Africa: Idaho National Laboratory INL/CON-06-11886, Oct 2006a.

Woo, Wanchuck, Hahn Choo, Donald W. Brown, and Zhili Feng. "Influence of the tool pin and shoulder on microstructure and natural aging kinetics in a friction-stir- processed 6061-T6 aluminum alloy." Metallurgical and Materials Transactions A (The Minerals, Metals \& Materials Society and ASM International 2007) Vol. 38A (January 2007): 6976.

Yang, Bangcheng, Junhui Yan, Michael A. Sutton, and Anthony P. Reynolds. "Banded microstructure in AA2024-T351 and AA2524-T351 aluminum friction stir welds." Materials Science \& Engineering (Elsevier Science Ltd) A364 (2004): 55-65. 


\title{
Microstructural Examination to Aid in Understanding \\ Friction Bonding Fabrication Technique \\ for Monolithic Nuclear Fuel
}

\author{
APPENDIX A \\ Literature Search on \\ RERTR Fuel Material Phenomena and Challenges
}

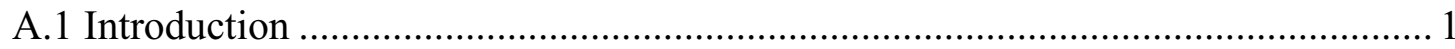

A.2 Uranium-Molybdenum Material Phases....................................................... 1

A. 3 Interface Reaction Layers .............................................................................. 3

A.3.1 Reaction Layer Formation and Effects ........................................................ 3

A.3.2 Reaction Layer Formed During Fabrication of Monolithic Fuel..................... 5

A.3.3 Reaction Layer Formed During Irradiation of Monolithic Fuel ..................... 6

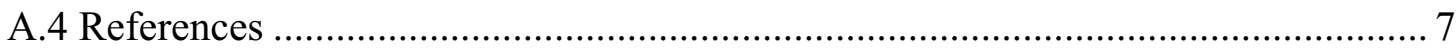

\section{A.1 Introduction}

This Appendix offers additional information found during the thesis literature search into the state of the art of the RERTR's fuel development efforts, specifically regarding fuel material phenomena and challenges related to:

1. UMo alloy material phases

2. U-Al interface reactions.

On one hand, these topics deserve some discussion as they are critical drivers for the RERTR program's overall fuel design and examination activities, and therefore comprise main themes in much of the literature. But on the other hand, these topics have little direct implication for this thesis research, and are therefore somewhat extraneous and distracting to the flow of information in the main report. The extent of their impact on the research is that:

1. Fuel plates from which the second group of samples was taken contained uranium foils that were alloyed with $10 \mathrm{wt} \%$ molybdenum. Although the molybdenum content had nothing to do with how the samples were prepared or with the examination that was performed on them, the following information gives insight into why the uranium is generally alloyed with molybdenum, and why a frequently preferred amount of molybdenum is about $10 \mathrm{wt} \%$.

2. The second group of samples was examined for any chemical reaction at their UAl interface. None was observed, but the following information helps explain why it was important to look for it.

\section{A.2 Uranium-Molybdenum Material Phases}

During irradiation, fission products form in the fuel matrix, causing the fuel to swell. When the fission products remain in solid or liquid state, the swelling is minimized, and is proportional only to the fuel's burnup $(\mathrm{BU})^{1}$. But swelling is greater if the fission products

\footnotetext{
1 "Burnup" (BU) refers to the percentage of the fuel's ${ }^{235} \mathrm{U}$ that is fissioned during irradiation.
} 


\section{APPENDIX A - Literature Search on RERTR Fuel Material Phenomena and Challenges}

convert to a gas state and especially if the gas coalesces into bubbles; the amount of gas formed and therefore the amount of additional swelling it causes is proportional to BU, temperature, and the molybdenum (Mo) content in the fuel alloy. (Kim, et al. 2007) ${ }^{2}$

Below $668^{\circ} \mathrm{C}$, pure uranium fuel typically has $\alpha$-phase material structure, with associated poor irradiation characteristics such as thermally-induced dimensional distortion, and swelling due to formation of bubbles of fission gas products. In contrast, a $\gamma$-phase uranium material structure is more dimensionally stable and inhibits bubble formation or migration. Thus alloys that keep the uranium in $\gamma$-phase are preferred; one such alloying material is Mo. Dispersion fuel irradiation tests revealed early-on that fission gas bubble formation at the fuel-aluminum interface is inversely proportional to the Mo content of the fuel. This was later recognized to be because the presence of Mo acts as a $\gamma$-phase stabilizer in the uranium. [(Clark, Meyer and Hayes 2002) and (Clark and Briggs 2004)]

Figure A-1 illustrates the structural phases that exist in UMo alloys at different temperatures and different Mo content. A UMo alloy will take on $\gamma$-phase structure if held for a time at temperatures in the $\gamma$-phase region; the alloy can then be quickly cooled (quenched) and will retain the $\gamma$-phase material structure indefinitely at room temperature. However, elevated temperatures can cause the material phase structure to change; this is why the UMo $\gamma$-phase is termed "metastable."

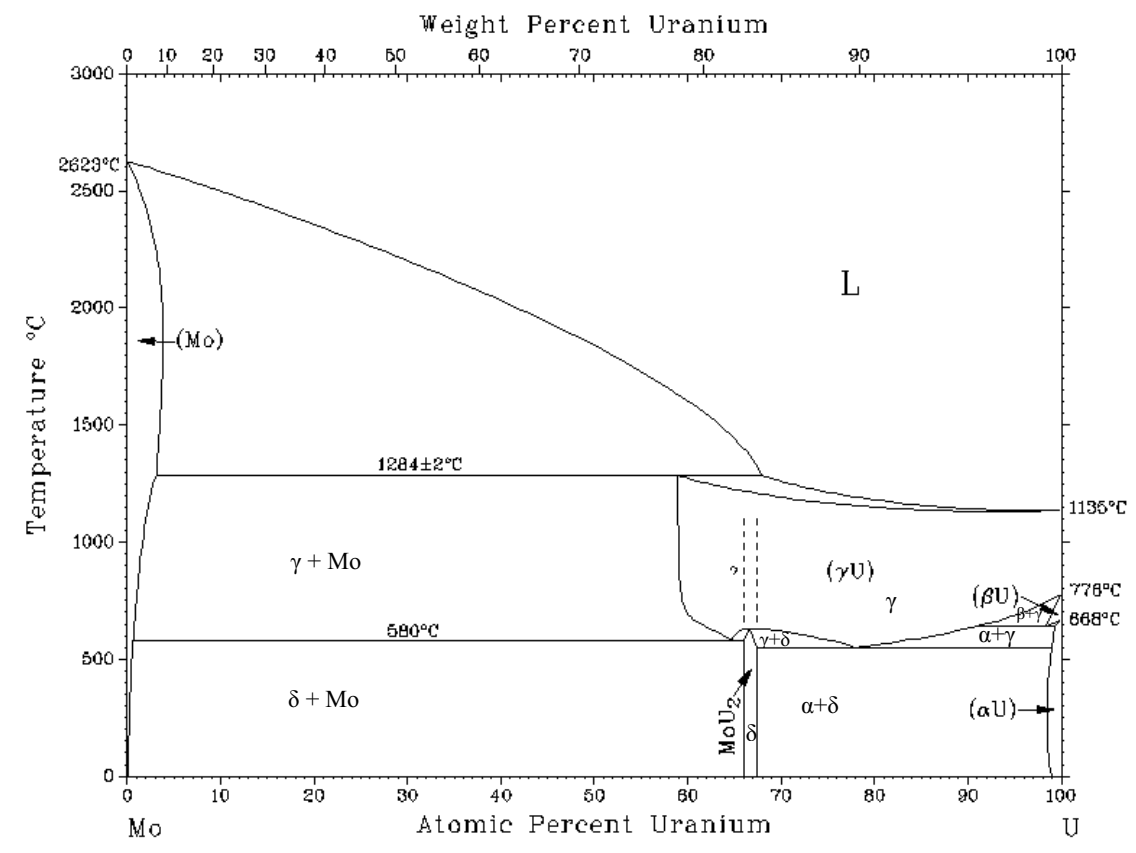

Figure A-1 Uranium-Molybdenum (U-Mo) binary phase diagram [after (Massalski 1996)].

\footnotetext{
${ }^{2}$ References are listed at the end of this appendix.
} 


\section{APPENDIX A - Literature Search on RERTR Fuel Material Phenomena and Challenges}

Note from Figure A-1 that at HIP fabrication temperature (around $580^{\circ} \mathrm{C}$ ), fuels of about $10 \mathrm{wt} \%$ Mo are more stable than fuels with other wt $\%$ Mo. The phase diagram shows a eutectoid ${ }^{3}$ at about $562^{\circ} \mathrm{C}$ and $10.5 \mathrm{wt} \% \mathrm{Mo}$ (i.e., $89.5 \mathrm{wt} \% \mathrm{U}$ ). At $580^{\circ} \mathrm{C}$, U7Mo and U8Mo are hypo-eutectoid in a two-phase region consisting of $(\alpha+\gamma)$-phase uranium. The $\mathrm{U} 10 \mathrm{Mo}$ is very near the boundary of the $\gamma$-phase and $(\alpha+\gamma)$-phase fields and is probably $\gamma$ phase. The U12Mo is in the $\gamma+\delta$ two-phase region. (Keiser, Jue and Burkes 2007) Thus U10Mo fuels subjected to HIP temperatures perform better than fuels with other Mo content.

UMo fuels are more resistant to swelling than $\alpha$-uranium-based fuels, because their $\gamma$ phase cubic structure (D. D. Keiser 2007) inhibits bubble formation or migration, so that fission product gases remain inherent in the fuel matrix. However, as the fuel is irradiated, the metastable $\gamma$-phase structure is disrupted and bubbles start to form and migrate. They are especially prone to coalesce at the fuel-aluminum interface if a reaction layer is present. Fuels subjected to high amounts of radiation and burn-up exhibit significantly more bubbles on the fuel-aluminum interface (Kim, et al. 2007).

The $\gamma$-phase is in a metastable state in UMo fuels, but they can remain in this state indefinitely at temperatures below the eutectoid. The highest temperature that these fuels see during irradiation is typically around $225^{\circ} \mathrm{C}$ (Finlay, Wachs and Robinson, et al. 2007); at these temperatures, the fuels remain $\gamma$-phase for indefinite times (D. D. Keiser 2007). It has been suggested that temperature annealing to form the $\gamma$-phase in UMo fuels could be a very effective method to reduce fission gas swelling during irradiation (Kim, et al. 2007). This seems likely as long as subsequent temperatures during fabrication or irradiation are kept low enough so that the material structure doesn't revert to $\alpha$-phase.

\section{A.3 Interface Reaction Layers}

\section{A.3.1 Reaction Layer Formation and Effects}

A tendency toward breakaway swelling of early UMo-Al dispersion fuels was observed by independent fuel development teams from several countries [(Jarousse, et al. 2006) and (Wachs, Keiser, et al. 2007)], and was recognized as the result of extensive chemical interaction between the fuel and aluminum matrix which occurred due to elevated irradiation temperature and BU (Wachs, Ambrosek, et al. 2006). This reaction layer phenomenon is also present in monolithic fuels (Kim, et al. 2007). Note that monolithic fuel has an advantage over dispersion fuel relative to reaction layer formation; in monolithic fuel, the interface area between the UMo fuel and aluminum (in both the matrix and the cladding) is orders of magnitude less than it is in dispersion fuel. (Clark and Briggs 2004)

The reaction layer, comprised of (UMo) $\mathrm{Al}_{\mathrm{x}}$ products (Jarousse, et al. 2006), is amorphous (non-crystalline) media where fission gas bubbles from the fuel can easily coalesce. Such a reaction layer is undesirable as it has several negative effects on the fuel's performance: it causes the fuel assembly to swell beyond its prescribed external

\footnotetext{
${ }^{3}$ A eutectoid reaction is an isothermal reversible reaction in which a solid solution is converted into two or more intimately mixed solids, the number of solids formed being the same as the number of components in the system.
} 


\section{APPENDIX A - Literature Search on RERTR Fuel Material Phenomena and Challenges}

dimensions; it forms a thermal barrier so that heat transfer out of the fuel is less efficient; fuel temperature increases, which speeds reaction layer formation and exacerbates bubble coalescence; and the reaction layer weakens the fuel-cladding bond, making it vulnerable to opening into a gap - such a gap would not only further decrease heat transfer, but could even lead to an "unzipping" failure mode in the fuel assembly. [(Clark and Briggs 2004), (Wachs, Keiser, et al. 2007), (Kim, et al. 2007), and (Burkes 2008)]

Aluminum reacts more readily with uranium in the $\alpha$-phase than it does with $\gamma$-phase uranium (D. D. Keiser 2007). Aluminum migration into the uranium does not stop just at the interface; aluminum precipitates have been found throughout the uranium fuel matrix in both U7Mo and U10Mo non-irradiated fuel that was annealed at $500^{\circ} \mathrm{C}$. (Keiser, Jue and Clark 2006) It is unclear what effect, if any, these precipitates have in the uranium, but so far foils with aluminum precipitates in them exhibit good irradiation performance. (Keiser, Robinson and Finlay 2007)

In efforts to decrease formation of the reaction layer, several nations have experiments underway on the use of silicon $(\mathrm{Si})$, zirconium $(\mathrm{Zr})$, titanium $(\mathrm{Ti})$, niobium $(\mathrm{Nb})$, or other materials to form diffusion barriers between the aluminum and the fuel. Application methods that have been or will be explored include alloying a material into the aluminum or uranium, cladding the uranium with material either before or after rolling the fuel foil, and applying a thermal spray or vapor deposition to the interface. [(Balart, et al. 2006), (Clark, Jue, et al. October 2006), (Wachs, Keiser, et al. 2007), and (Park, et al. October 2006)] The material application methods have a variety of implications for the various monolithic fuel fabrication techniques.

So far the incorporation of silicon ( $\mathrm{Si}$ ) seems very promising in both monolithic and dispersion fuels, either as a discrete diffusion barrier or alloyed with the aluminum. Addition of $\mathrm{Si}$ to the $\mathrm{Al}$ matrix in dispersion fuel greatly reduced growth of the reaction layer and increased irradiation stability. Both RERTR-6 and RERTR-7 showed that incorporation of $2 \% \mathrm{Si}$ or more into the dispersion fuel meat's aluminum matrix substantially reduced U-Al interaction, and that stability of the modified interaction layer exceeded that of the UMo fuel itself. This occurred in part because silicon accumulates in the interaction layer, promoting a crystalline rather than amorphous structure, and thus reducing buildup of gas bubbles. The accumulated Si also acts as a diffusion barrier to the aluminum so that significantly less U-Al reaction can continue. Higher temperatures require more $\mathrm{Si}$ to provide this protection, as the $\mathrm{Si}$ is consumed in producing increased amounts of the stable and relatively benign reaction product. [(Balart, et al. 2006), (Wachs, Keiser, et al. 2007), (Park, et al. October 2006), (Kim, et al. 2007), and (Burkes 2008)]

Interestingly, it appears that the Mo in the $U$ fuel serves another benefit beyond stabilization of the $\gamma$-phase; it also greatly reduces growth of the reaction layer, in part by increasing the Si's effectiveness. When the Mo content in a UMo alloy is increased from 7 to $10 \mathrm{wt} \%$, then significantly more of the Si-enriched layers develop. Not only does the high Si concentration reduce Al diffusion into the UMo alloy, but also interaction between Mo and Si inhibits Si diffusion deeper into the UMo solid solution, thereby improving its ability to inhibit Al diffusion even more. (Keiser, Jue and Clark 2006) 


\section{APPENDIX A - Literature Search on RERTR Fuel Material Phenomena and Challenges}

Annealing experiments (Keiser, Jue and Clark 2006) on non-irradiated fuel-aluminum couples and on non-irradiated FB fuel plates (Finlay, Wachs and Hofman 2006) indicate that at relatively high temperatures $\left(\sim 500^{\circ} \mathrm{C}\right)$ for short anneal times $(30 \mathrm{~min})$, interaction layer formation is very different between U7Mo and U10Mo. The U7Mo-Al reaction layers were much thicker, and the U10Mo-Al reaction layers were higher-Si, which inhibits U-Al interaction. However, the (Keiser, Jue and Clark 2006) experiments showed that, even for the U10Mo interaction layer, the diffusion barrier resulting from the presence of Si in the aluminum loses its effect over longer annealing time periods. After longer timeframes at this high temperature, the final diffusion structures are very similar to those observed in typical UMo-Al diffusion couples. If the annealing temperatures were much lower, the $\mathrm{Si}$ in the interaction layers may stay enriched for longer annealing times. Or, if the $\mathrm{Si}$ was at a higher concentration (at least greater than $1 \mathrm{wt} \%$ ) in the $\mathrm{Al}$ alloy, the Si-enriched layers may remain stable for longer annealing times at the high temperature. (Hofman, et al. May 2006) recommends at least $5 \mathrm{wt} \%$ Si should be present in the Al matrix of dispersion fuels in order to stabilize a $\mathrm{U}(\mathrm{Al}, \mathrm{Si})_{3}$ reaction product in irradiated fuels over the Al-rich products that tend to develop. Of course, irradiation temperatures would typically be much lower than the $500^{\circ} \mathrm{C}$ employed for the annealing studies reported by (Keiser, Jue and Clark 2006) and (Finlay, Wachs and Hofman 2006).

(Clark, Jue, et al. October 2006) report that a $100 \mu \mathrm{m}$ thick $\mathrm{Zr}$ diffusion barrier used in a HIP surrogate fuel plate had a good bond with the aluminum cladding but interacted with and separated from the UMo foil. However, PIE metallography of Zircaloy-clad hotrolled monolithic fuel (fabricated by CNEA and irradiated in the ATR) shows low and uniform swelling with little or no reaction product and no bubble formation between the Zr cladding and the U7Mo foil (Robinson and Finlay September 2007). (Park, et al. October 2006) observed that when Zr was alloyed into UMo, it reduced the reactor layer's growth rate, and did so even better when $\mathrm{Si}$ was also added to the $\mathrm{Al}$; however, it also reduced the $\gamma$-phase stability of U7Mo.

\section{A.3.2 Reaction Layer Formed During Fabrication of Monolithic Fuel}

Different thicknesses of reaction layer are formed at the monolithic fuel UMo-Al interface, depending on the fuel fabrication technique and the amount of Mo alloyed into the U. Reaction layer thicknesses between Al clad and UMo foils were measured on nonirradiated samples ${ }^{4}$ :

- $\quad$ TLPB - Fuel samples were fabricated by the TLPB technique, with an Al-Si material inserted at the interface, heated to $590^{\circ} \mathrm{C}$ for $15 \mathrm{~min}$, at $6.9 \mathrm{MPa}$ pressure:

○ Reaction layers $10-40 \mu \mathrm{m}$ thick with high Si content; thickness depended on the amount of Si applied to the interface.

- HIP - Fuel samples were fabricated by HIP performed at $580^{\circ} \mathrm{C}$ for 90 min at 103 $\mathrm{MPa}(15,000 \mathrm{psi})$ pressure:

○ U7Mo foil was fully consumed

- U8Mo foil was about $2 / 3$ consumed ( $1 / 3$ on each side of the foil), with a reaction layer thickness of about $250 \mu \mathrm{m}$ thick

\footnotetext{
${ }^{4}$ Examination data on as-fabricated, non-irradiated samples were gathered from references (Clark and Briggs 2004), (Clark, Jue, et al. October 2006), (Burkes, Keiser, et al. March 2007), (Keiser, Jue and Burkes 2007), and (Finlay, et al. 2007).
} 


\section{APPENDIX A - Literature Search on RERTR Fuel Material Phenomena and Challenges}

○ U10Mo foil had 2-10 $\mu \mathrm{m}$ reaction layer at U10Mo-Al interface

- U12Mo foil had a reaction layer "a few microns thick"

- Hardness of U-Al reaction layers measured after HIP were much harder (617 and $\left.653 \mathrm{H}_{\mathrm{V}}\right)$ than that of the UMo foil $\left(263 \mathrm{H}_{\mathrm{V}}\right)$ or the Al6061 cladding $\left(47 \mathrm{H}_{\mathrm{V}}\right)$.

- FB - Fuel samples fabricated by FB show that in general no reaction product forms at the interface:

- One FB plate (fabrication parameters were unspecified) had no reaction layer at the U10Mo-Al interface until annealing at $500^{\circ} \mathrm{C}$ for $30 \mathrm{~min}$, after which a reaction layer $\sim 3 \mu \mathrm{m}$ thick was evident at the U10Mo-Al interface. This layer appeared stable with no regions of breakaway interaction or signs of debonding. (Annealing is no longer typically performed on FB plates.)

- Another FB plate fabricated with tool load roughly estimated at $17.8 \mathrm{kN}(4,000$ $\mathrm{lbf}$ ), and with plate temperature estimated at $400-500^{\circ} \mathrm{C}$ for a few seconds, had no reaction layer at the $\mathrm{U} 10 \mathrm{Mo}-\mathrm{Al}$ interface.

\section{A.3.3 Reaction Layer Formed During Irradiation of Monolithic Fuel}

To help understand radiation effects on monolithic fuels, PIE reaction layer thickness measurements for monolithic fuel plate samples that were irradiated in RERTR-6 (moderate power density and temperature, $\sim 45 \% \mathrm{BU}$ ) and RERTR-7 (moderately high power density and temperature, $\sim 80 \% \mathrm{BU}$ ) are given here. ${ }^{5}$ No PIE data are available yet for later RERTR experiments, including HIP fuel plates. The FB plates irradiated in RERTR-6 and RERTR-7 were all fabricated with a steel-faced tool, although the pin diameter was changed. Also, RERTR-6 FB fuel plates were annealed at $500^{\circ} \mathrm{C}$ for $30 \mathrm{~min}$ after $\mathrm{FB}$, and so they might have had a reaction layer existing prior to irradiation. Note that during irradiation in the ATR, one edge of fuel plates are typically oriented toward the core, and the other away, with a consequent self-shielding effect: therefore a large $\Delta$ flux exists between the edges $(\Delta$ power $=2 \mathrm{X})$.

- TLPB U12Mo

○ RERTR-7: reaction layer of 10-40 $\mu$ m thickness at the U-Al interface, with little or no evidence of gas bubbles or consequent delamination. However, cracks formed at the Al-Al interface (at the edge oriented toward the core) in all TLPB fuel plates, and caused one plate to break open during reactor operation.

- FB U7Mo

○ RERTR-6: reaction layer 4-6 $\mu$ m thick, with some gas bubbles.

- FB U10Mo

O RERTR-6: reaction layer $\sim 3 \mu \mathrm{m}$ thick on the edge of the foil oriented away from the core, and $\sim 18 \mu \mathrm{m}$ thick on the edge toward the core. No gas bubbles were evident.

O RERTR-7: reaction layer $\sim 3 \mu \mathrm{m}$ thick on the edge of the foils oriented away from the core, and $\sim 6 \mu \mathrm{m}$ thick on the edge toward the core. Gas bubbles formed at the interface, and one side of the bond delaminated when the sample was cut for PIE (typical for two fuel plates).

\footnotetext{
${ }^{5}$ PIE data on irradiated samples were gathered from references (Burkes, Hallinan, et al. January 2007b), (Finlay, et al. 2007), (Keiser, Robinson and Finlay 2007), and (Robinson and Finlay September 2007).
} 


\section{APPENDIX A - Literature Search on RERTR Fuel Material Phenomena and Challenges}

- FB U12Mo

O RERTR-7: reaction layer 3-7 $\mu \mathrm{m}$ thick on the edge of the foils oriented away from the core, and 5-12 $\mu \mathrm{m}$ thick on the edge toward the core. Some gas bubbles formed at the interface edge oriented toward the core.

\section{A.4 $\underline{\text { References }}$}

Balart, S., et al. "Progress on RERTR activities in Argentina." International Meeting on Reduced Enrichment for Research and Test Reactors (RERTR) - 2006. Cape Town, South Africa, 2006.

Burkes, Douglas E. Verbal communication during Jan.-Mar. 2008.

Burkes, Douglas E., Dennis D. Keiser, Daniel M. Wachs, J.S. Larson, and Michael D. Chapple. "Characterization of monolithic fuel foil properties and bond strength." International Topical Meeting on Research Reactor Fuel Management (RRFM) 2007. Lyon, France: Idaho National Laboratory INL/CON-07-12208, March 2007.

Burkes, Douglas E., N. Patrick Hallinan, Curtis R. Clark, Glenn A. Moore, and Michael D. Chapple. "Update on friction stir welding." RERTR Independent Program Review meeting. Unpublished presentation given at the Idaho National Laboratory, January $2007 b$.

Clark, Curtis R., and R.J. Briggs. "Development of a monolithic research reactor fuel type at Argonne National Laboratory." Americas Nuclear Energy Symposium. Miami, FL, 2004.

Clark, Curtis R., Jan-Fong Jue, Glenn A. Moore, N. Patrick Hallinan, and Blair H. Park. "Update on monolithic fuel fabrication methods." International Meeting on Reduced Enrichment for Research and Test Reactors (RERTR) - 2006. Cape Town, South Africa: Idaho National Laboratory INL/CON-06-11897, October 2006.

Clark, Curtis R., Mitchell K. Meyer, and Steven L. Hayes. "RERTR fuel development at Argonne National Laboratory." Americas Nuclear Energy Symposium. Miami, FL, 2002.

Finlay, M. Ross, Daniel M. Wachs, Adam Robinson, and G.L. Hofman. "Post irradiation examination (PIE) of monolithic mini-fuel plates from RERTR-6 \& 7."

International Topical Meeting on Research Reactor Fuel Management (RRFM) 2007. Lyon, France, 2007.

Finlay, M. Ross, Daniel M. Wachs, and G.L. Hofman. "Post irradiation examination (PIE) of monolithic mini fuel plates from RERTR-6." International Meeting on Reduced Enrichment for Research and Test Reactors (RERTR) - 2006. Cape Town, South Africa, 2006.

Hofman, G.L., Y.S. Kim, H.J. Ryu, and J. Rest. "Attempt to solve the instability in the irradiation behaviour of low enriched U-Mo/Al dispersion fuel," RRFM-2006." International Topical Meeting on Research Reactor Fuel Management (RRFM) 2006. Sofia, Bulgaria, May 2006. 


\section{APPENDIX A - Literature Search on RERTR Fuel Material Phenomena and Challenges}

Jarousse, C., P. Lemoine, P. Boulcourt, W. Petry, and A. Rohrmoser. "Last manufacturing results of monolithic UMo full size prototype plates." International Meeting on Reduced Enrichment for Research and Test Reactors (RERTR) - 2006. Cape Town, South Africa, 2006.

Keiser, Dennis D, Adam B. Robinson, and M. Ross Finlay. "Observations derived from the characterization of monolithic fuel plates irradiated as part of the RERTR-6 experiment." International Meeting on Reduced Enrichment for Research and Test Reactors (RERTR) - 2007. Prague, Czech Republic, 2007.

Keiser, Dennis D, Jan-Fong Jue, and Douglas E. Burkes. "Characterization and testing of monolithic RERTR fuel plates." International Topical Meeting on Research Reactor Fuel Management (RRFM) - 2007. Lyon, France: Idaho National Laboratory INL/CON-07-12224, 2007.

Keiser, Dennis D. "Interdiffusion behavior in $\gamma$-phase U-Mo alloy versus Al-6061 alloy couples fabricated by friction stir welding." Defect and Diffusion (Trans Tech Publications, Switzerland) Vol. 266 (2007): 131-148.

Keiser, Dennis D., Jan-Fong Jue, and Curtis R. Clark. "UMo foil/cladding interactions in friction stir welded monolithic RERTR fuel plates." International Meeting on Reduced Enrichment for Research and Test Reactors (RERTR) - 2006. Cape Town, South Africa: Idaho National Laboratory INL/CON-06-11914, 2006.

Kim, Yeon Soo, G.L. Hofman, P.G. Medvedev, G.V. Shevlyakov, A.B. Robinson, and H.J. Ryu. "Post irradiation analysis and performance modeling of dispersion and monolithic U-Mo fuels." International Meeting on Reduced Enrichment for Research and Test Reactors (RERTR) - 2007. Prague, Czech Republic, 2007.

Massalski, T.B., ed. Binary Alloy Phase Diagrams. Vol. III. ASM International, MAterials Park, OH, 1996.

Park, Jong Man, et al. "Interdiffusion behaviors of U-Mo-Zr/Al-Si." International Meeting on Reduced Enrichment for Research and Test Reactors (RERTR) - 2006. Cape Town, South Africa, October 2006.

Robinson, Adam B., and M. Ross Finlay. RERTR-7 Post Irradiation Examination (PIE). External Report, Idaho National Laboratory INL/EXT-07-13271, September 2007.

Wachs, Daniel M., et al. "Progress in the development of LEU fuel." Global 2007 Conference - Advanced Nuclear Fuel Cycles and Systems. Boise, ID, 2007.

Wachs, Daniel M., Richard G. Ambrosek, Gray S. Chang, and Mitchell K. Meyer. "Design and status of RERTR irradiation tests in the advanced test reactor (ATR)." International Meeting on Reduced Enrichment for Research and Test Reactors (RERTR) - 2006. Cape Town, South Africa: Idaho National Laboratory INL/CON06-11886, 2006. 


\title{
Microstructural Examination to Aid in Understanding \\ Friction Bonding Fabrication Technique \\ for Monolithic Nuclear Fuel
}

\begin{abstract}
APPENDIX B
Photos of Fuel Plate Fabrication, Sample Preparation, and Examination Equipment
\end{abstract}

Figure

B-1 FASB Rolling Machine

B-2 Current Friction Bonding Machine at FASB

B-3 New Friction Bonding Machine at FASB

B-4 Aluminum Plates After Friction Bonding

B-5 FASB Light Table

B-6 Shear in FASB West Radiation Hood

B-7 FASB High-Speed Saw

B-8 Sample Mounting Epoxy Kit

B-9 EML Sample Polishing Stations

B-10 EML Sample Etching Stations

B-11 EML Computer, Optical Microscopes, and Microhardness Tester 
APPENDIX B - Photographs of Sample Preparation \& Examination Facilities

(page is intentionally blank) 
APPENDIX B - Photographs of Sample Preparation \& Examination Facilities

\section{B-1 FASB Rolling Machine}

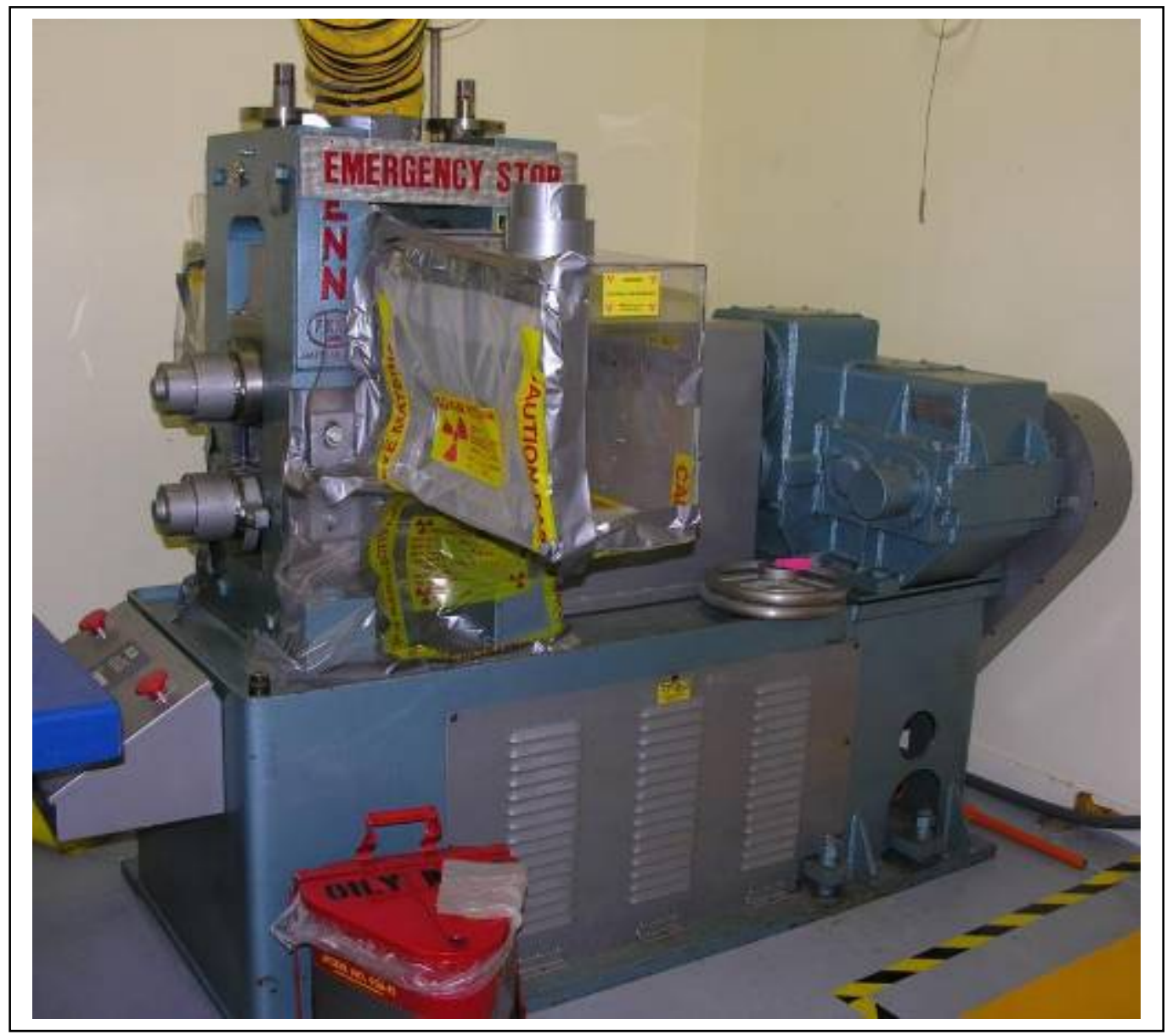

Figure B-1. Rolling machine used to cold-roll UMo foils in the FASB facility. (FENN International Rolling Mills IRM, Pawtucket, RI, Serial No. 006655) 
APPENDIX B - Photographs of Sample Preparation \& Examination Facilities

\section{B-2 Current Friction Bonding Machine at FASB}

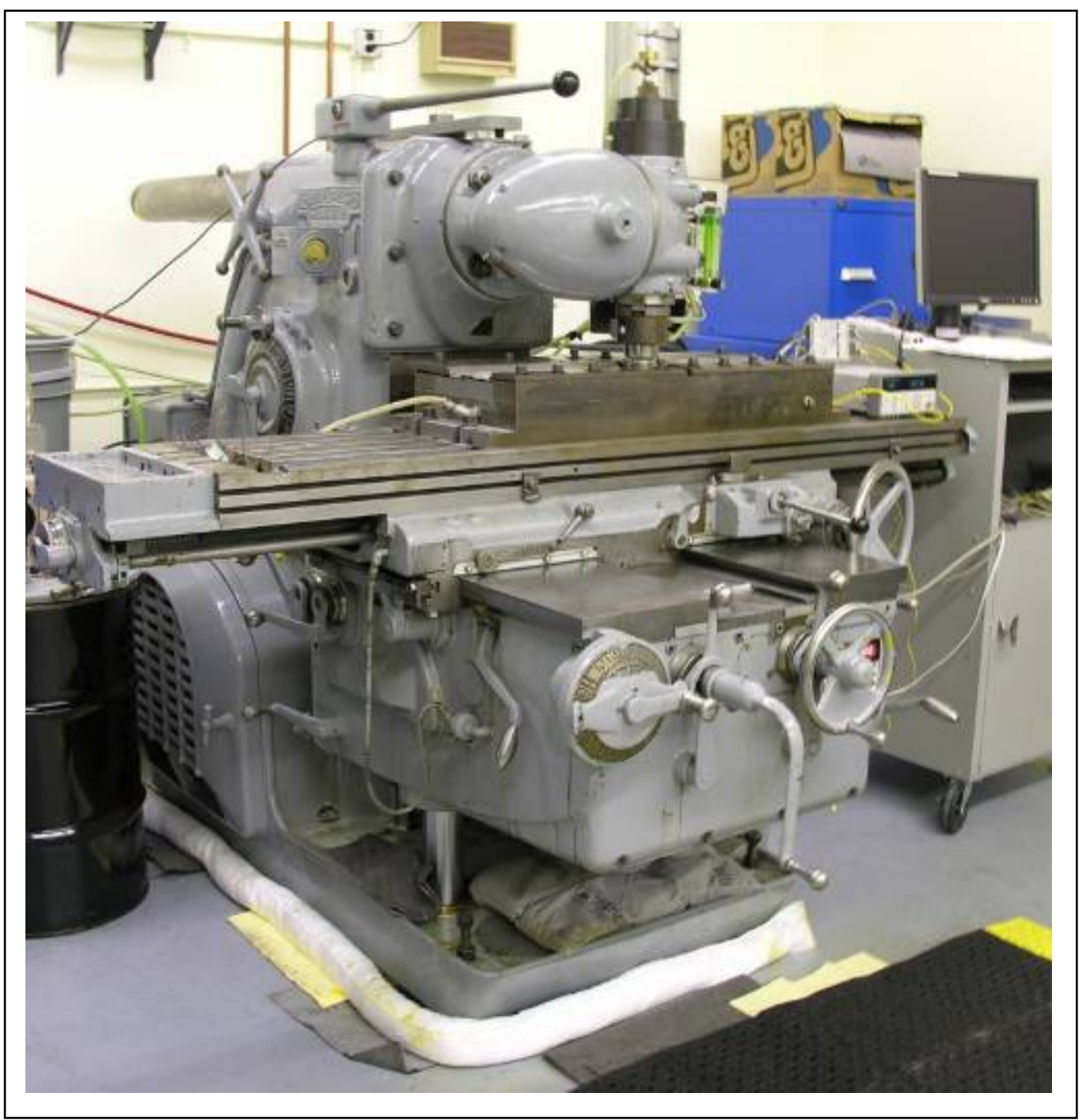

Figure B-2.a Milling machine currently used to perform friction bonding on nuclear fuel plates in the FASB facility. (Kearney \& Trecker Corp., Milwaukee, WI, Model 330, No. 3-30hp DP, TF Series, Serial No. 7-8118) 
APPENDIX B - Photographs of Sample Preparation \& Examination Facilities

\section{B-2 Current Friction Bonding Machine at FASB (continued)}

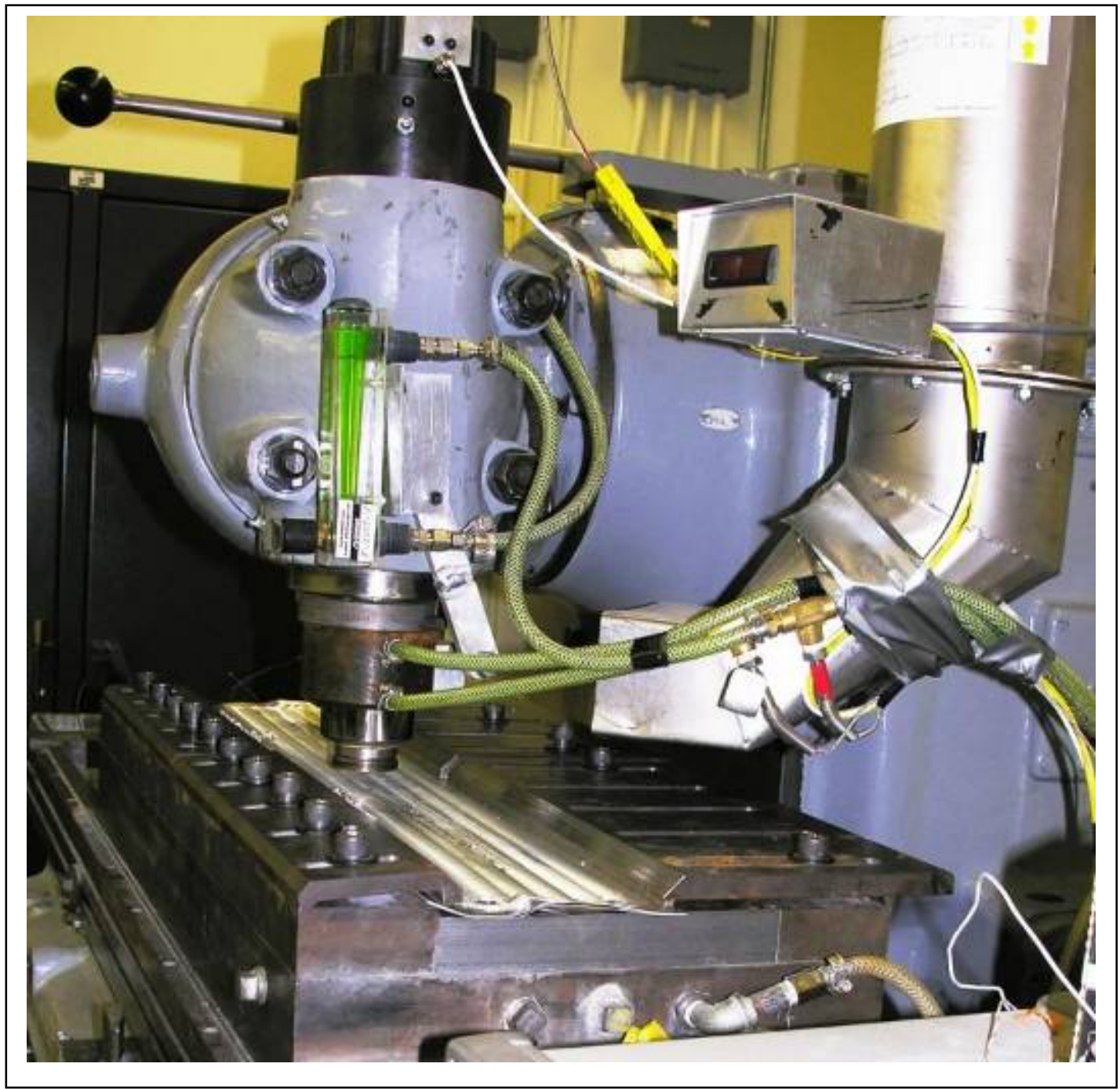

Figure B-2.b Kearney \& Trecker milling machine performing friction bonding on nuclear fuel plates in the FASB facility. 
APPENDIX B - Photographs of Sample Preparation \& Examination Facilities

\section{B-2 Current Friction Bonding Machine at FASB (continued)}

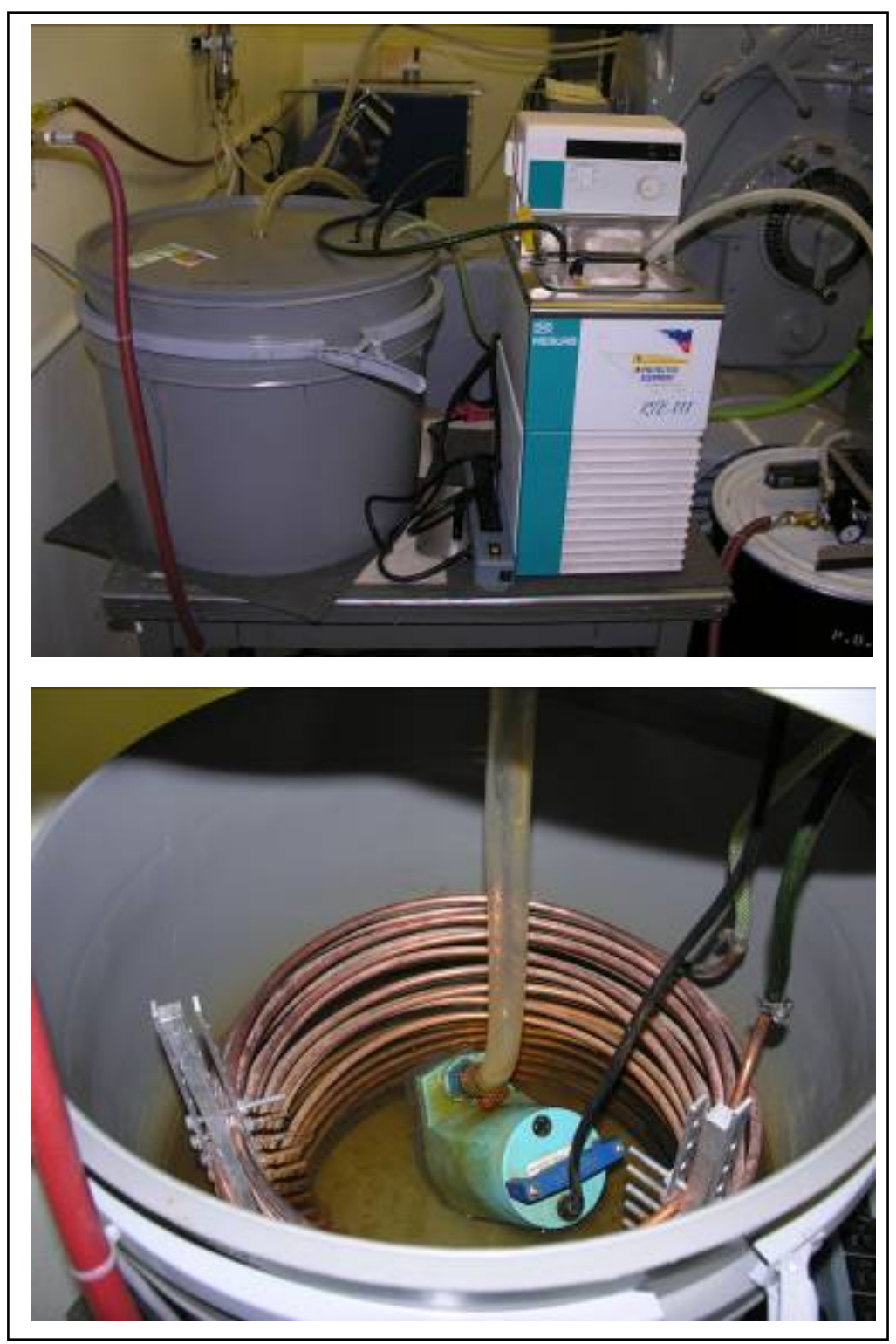

Figure B-2.c Cooling system with tank, heat exchanger coils, and pumps, feeds ethylene glycol through the FB tool on the Kearney \& Trecker milling machine FB tool. [Ref. 8] 
APPENDIX B - Photographs of Sample Preparation \& Examination Facilities

\section{B-3 New Friction Bonding Machine at FASB}

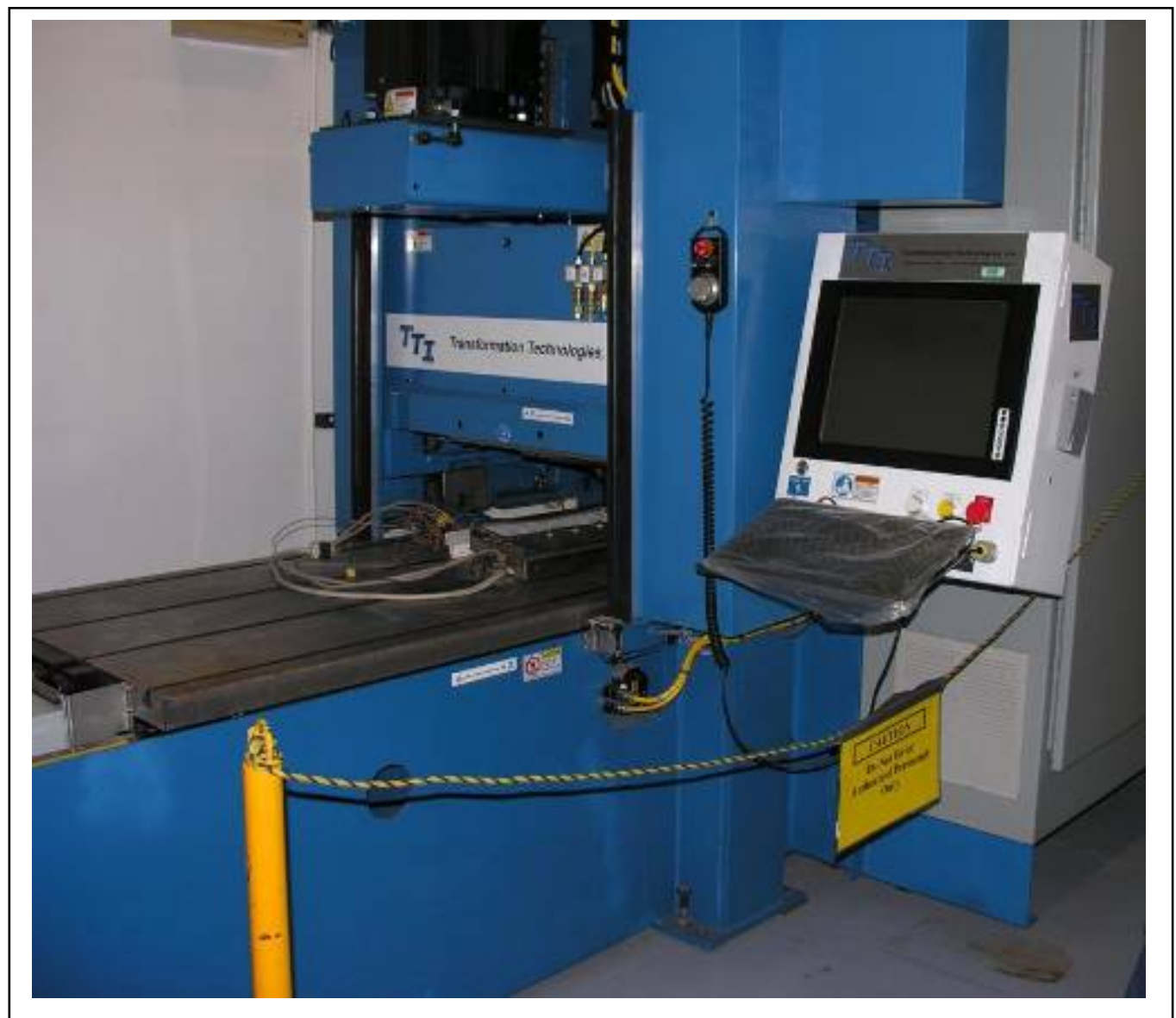

Figure B-3. New TTI machine planned for future friction bonding activities in the FASB facility. 
APPENDIX B - Photographs of Sample Preparation \& Examination Facilities

\section{B-4 Aluminum Plate After Friction Bonding}

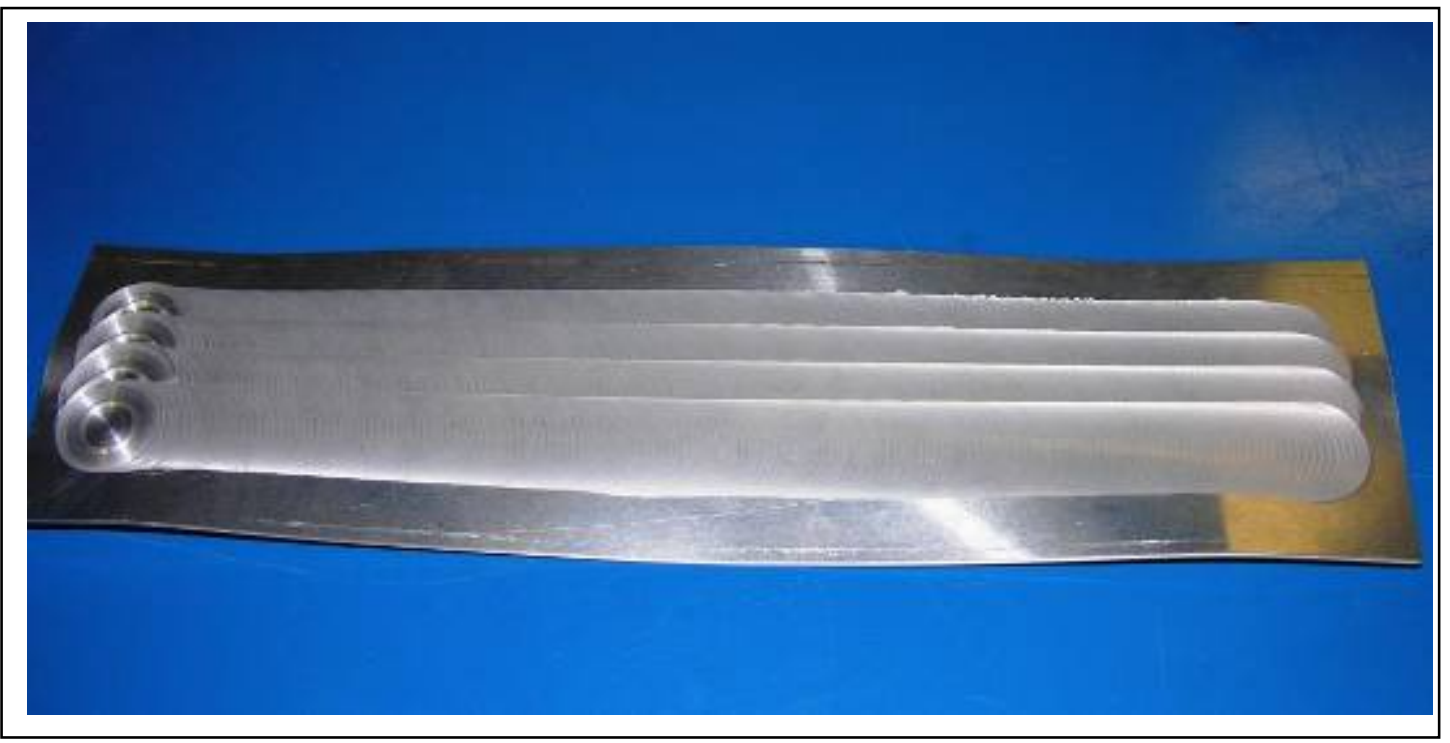

Figure B-4. Aluminum plate friction bonded at FASB. 
APPENDIX B - Photographs of Sample Preparation \& Examination Facilities

\section{B-5 FASB Light Table}

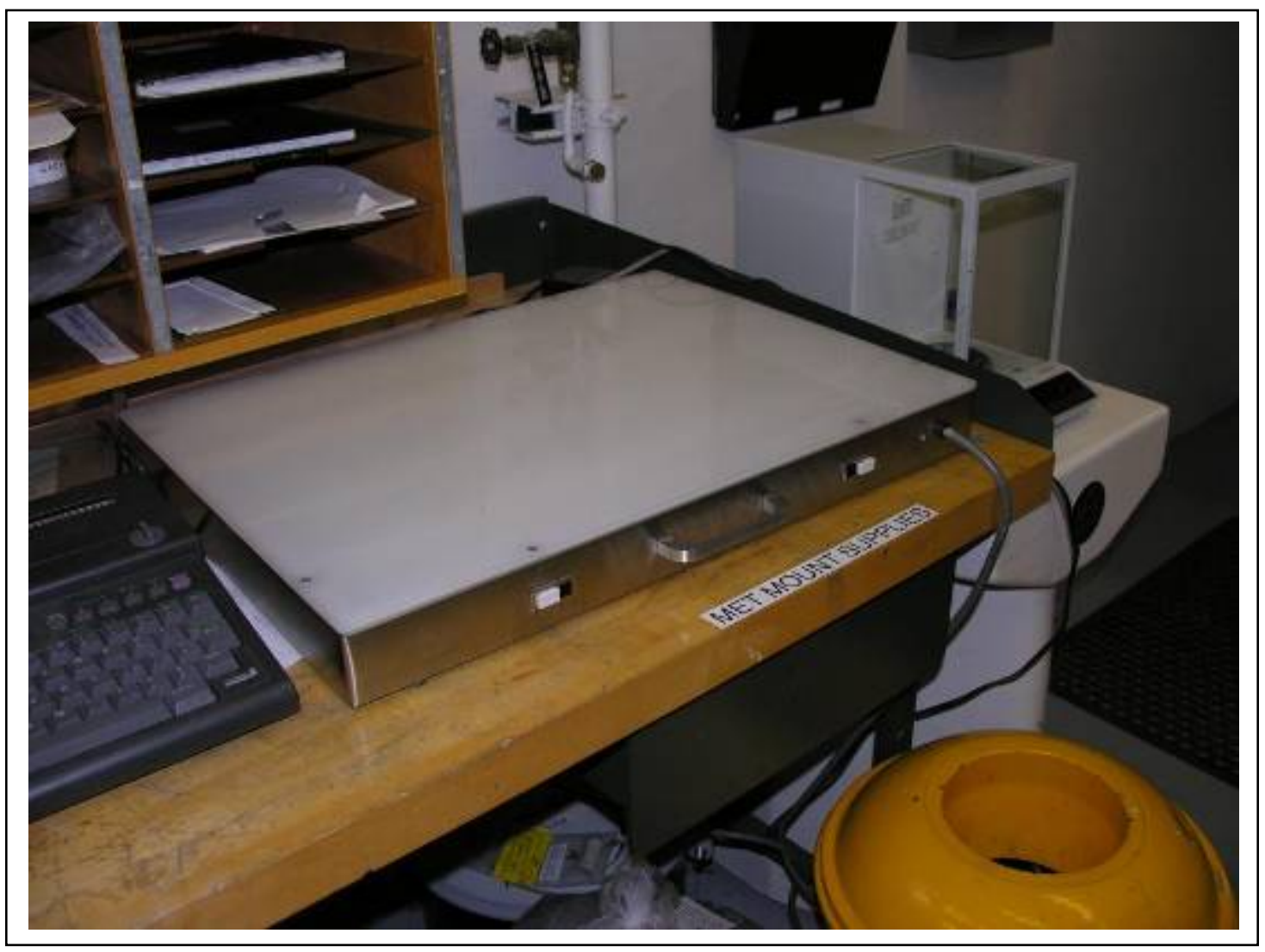

Figure B-5. Light table in the FASB facility, used for close inspection of neutron radiographs, to determine where to cut samples from fuel plates. 
APPENDIX B - Photographs of Sample Preparation \& Examination Facilities

\section{B-6 Shear in FASB West Radiation Hood}

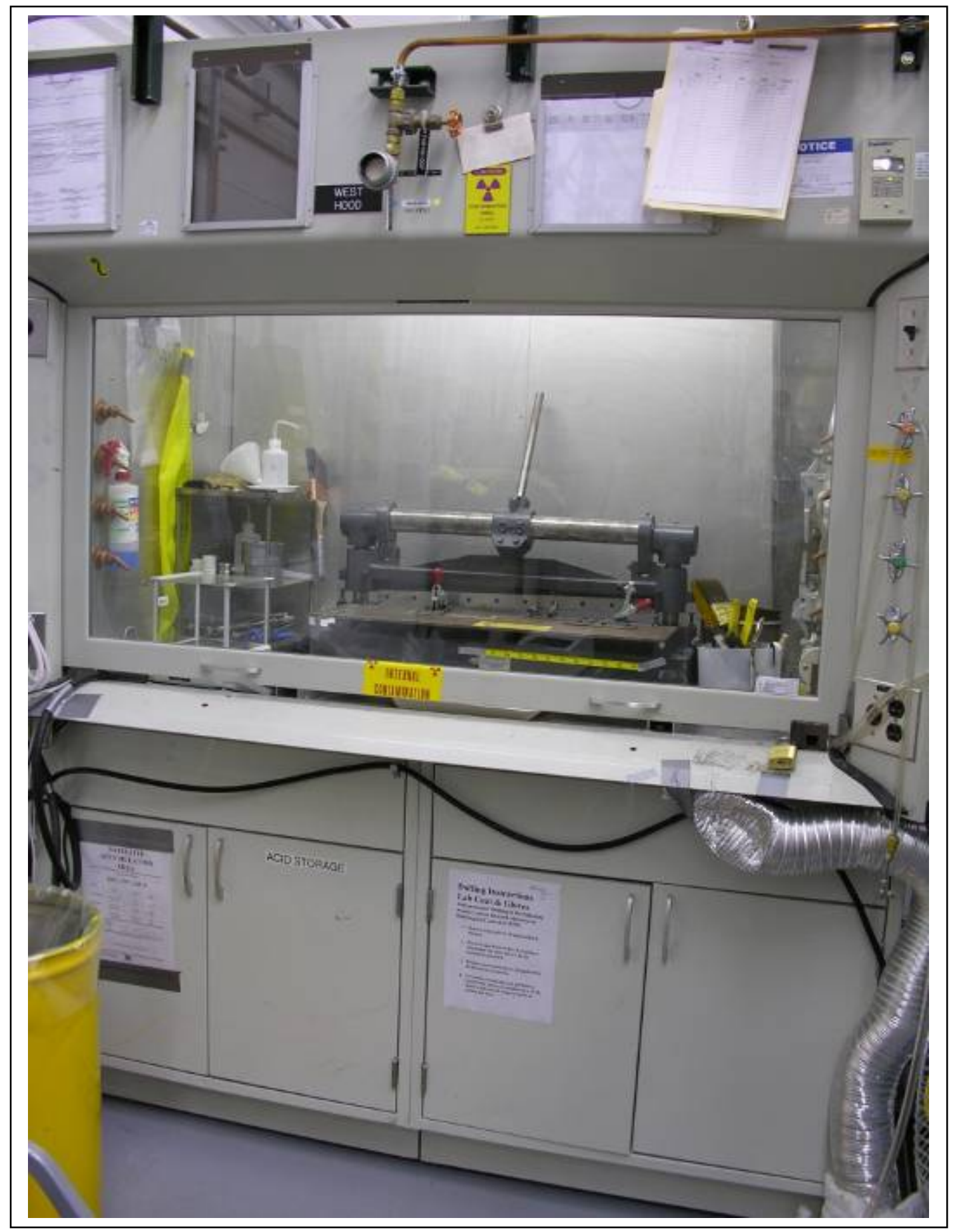

Figure B-6. Shear inside west radiation hood in the FASB facility. 
APPENDIX B - Photographs of Sample Preparation \& Examination Facilities

B-7 FASB High-Speed Saw

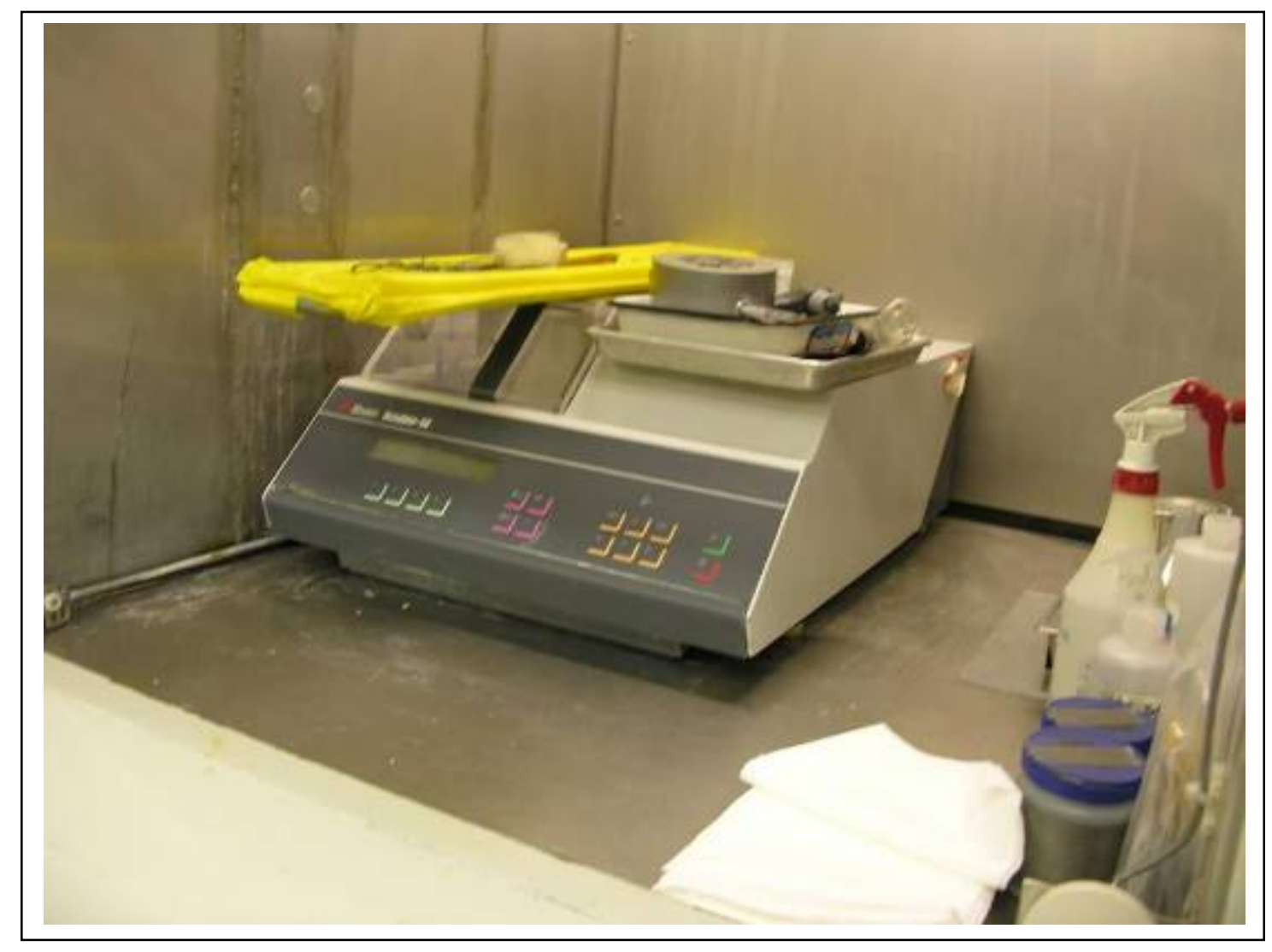

Figure B-7.a High speed saw inside east radiation hood in the FASB facility. Fuel plates, wrapped in yellow plastic, are on top of the saw cabinet. 
APPENDIX B - Photographs of Sample Preparation \& Examination Facilities

\section{B-7 FASB High-Speed Saw (continued)}

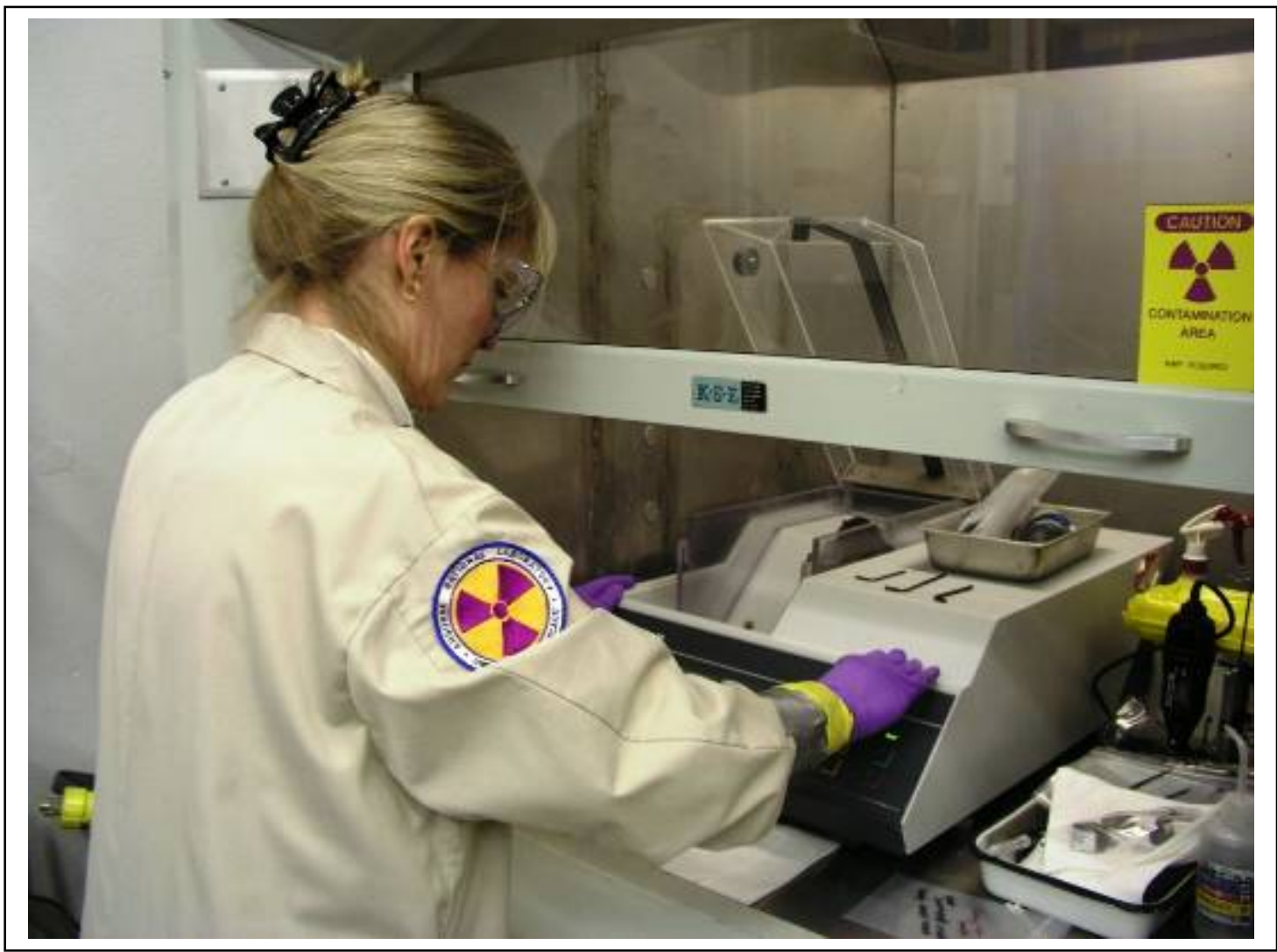

Figure B-7.b Cutting a sample using the high-speed saw in the east radiation hood in the FASB facility. 
APPENDIX B - Photographs of Sample Preparation \& Examination Facilities

\section{B-8 Sample Mounting}

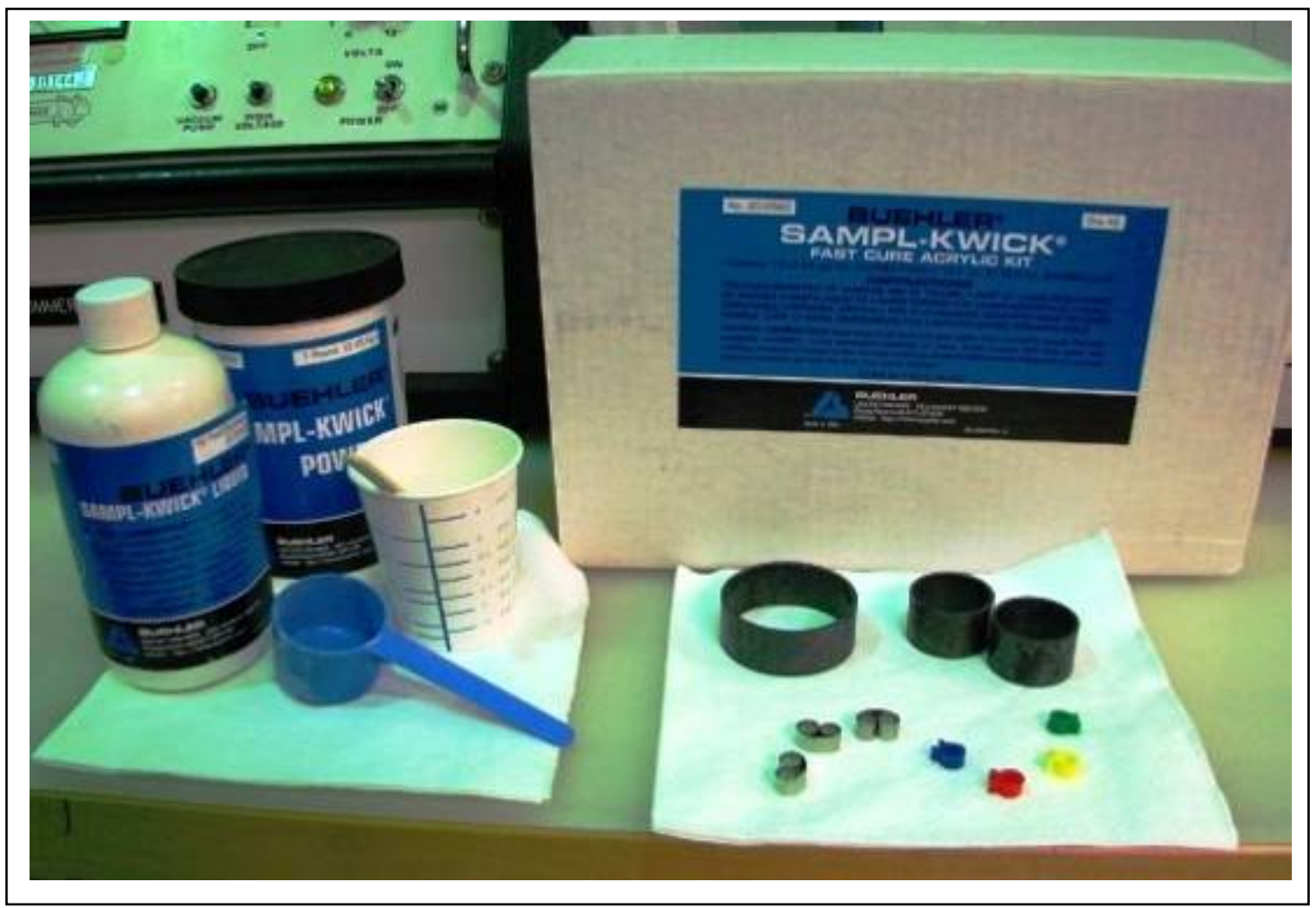

Figure B-8. Kit of epoxy quick-mixing materials and equipment. Cut samples are set into clips (either metal or colored plastic) to hold them upright, and then into sample rings with the surface of interest downward. Liquid and powder from the kit are mixed to form epoxy, which is immediately poured into the rings over and around the clipped samples, to create met mounts. The epoxy hardens to transparent in about 15 minutes. 
APPENDIX B - Photographs of Sample Preparation \& Examination Facilities

\section{B-9 EML Sample Polishing Stations}

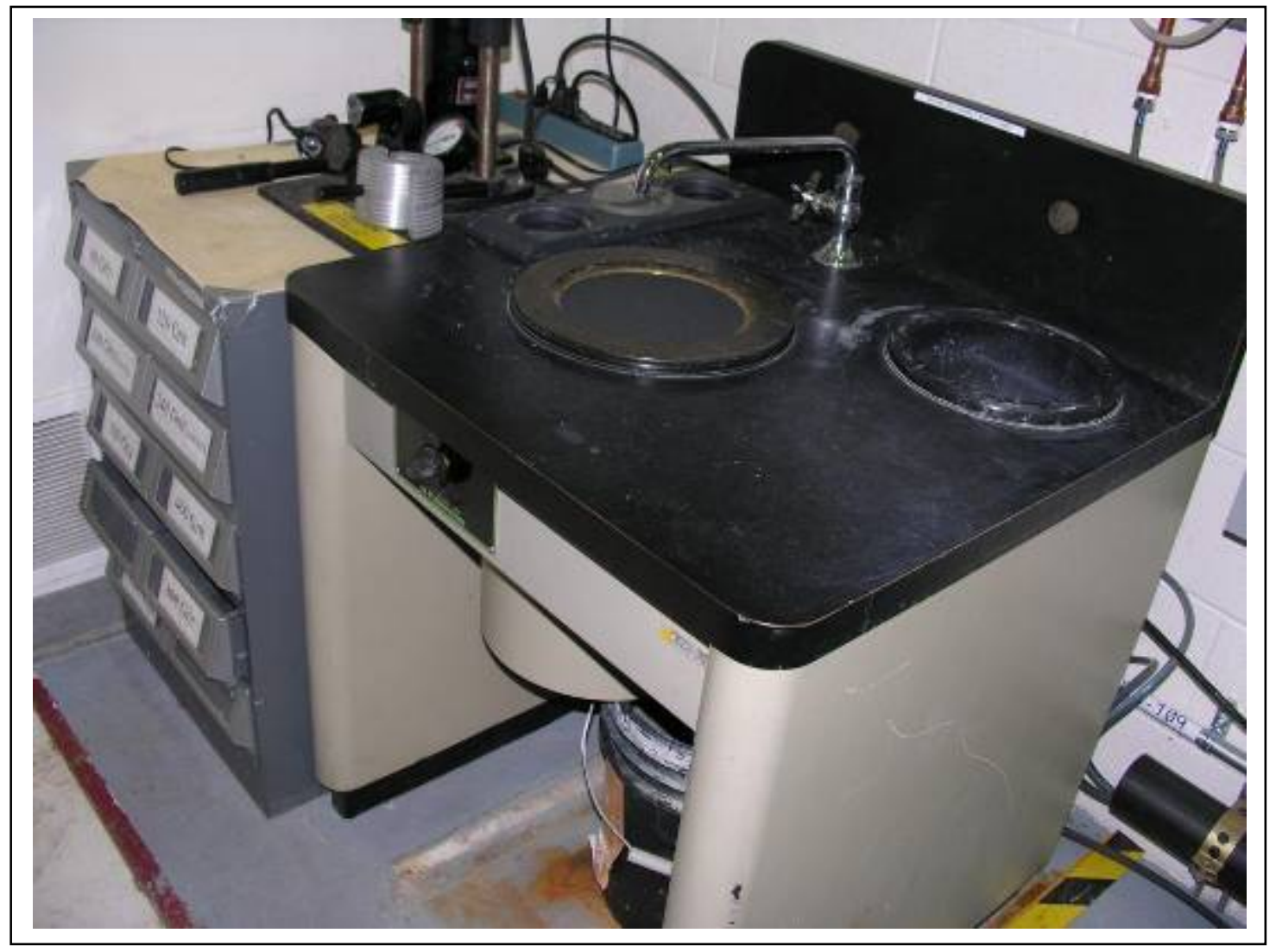

Figure B-9.a Polishing wheel station, where polishing of samples with no nuclear material is performed in EML. Drawers contain sandpaper circles with surfaces of varying grit sizes. 
APPENDIX B - Photographs of Sample Preparation \& Examination Facilities

\section{B-9 EML Sample Polishing Stations (continued)}

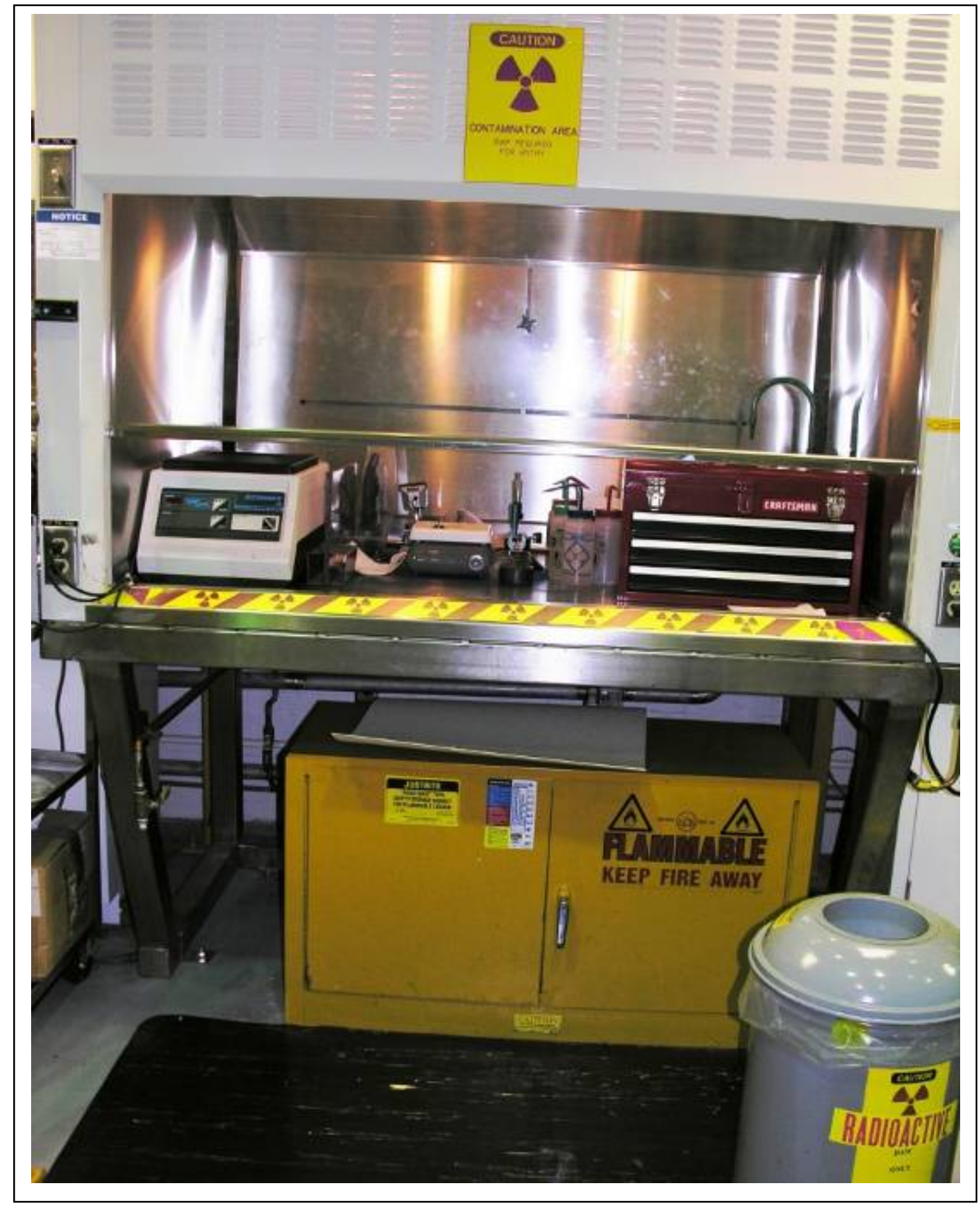

Figure B-9.b Second fume hood (radioactive) in EML, where hand polishing is performed on nuclear materials, including epoxied samples containing depleted uranium. 
APPENDIX B - Photographs of Sample Preparation \& Examination Facilities

\section{B-10 EML Sample Etching Stations}

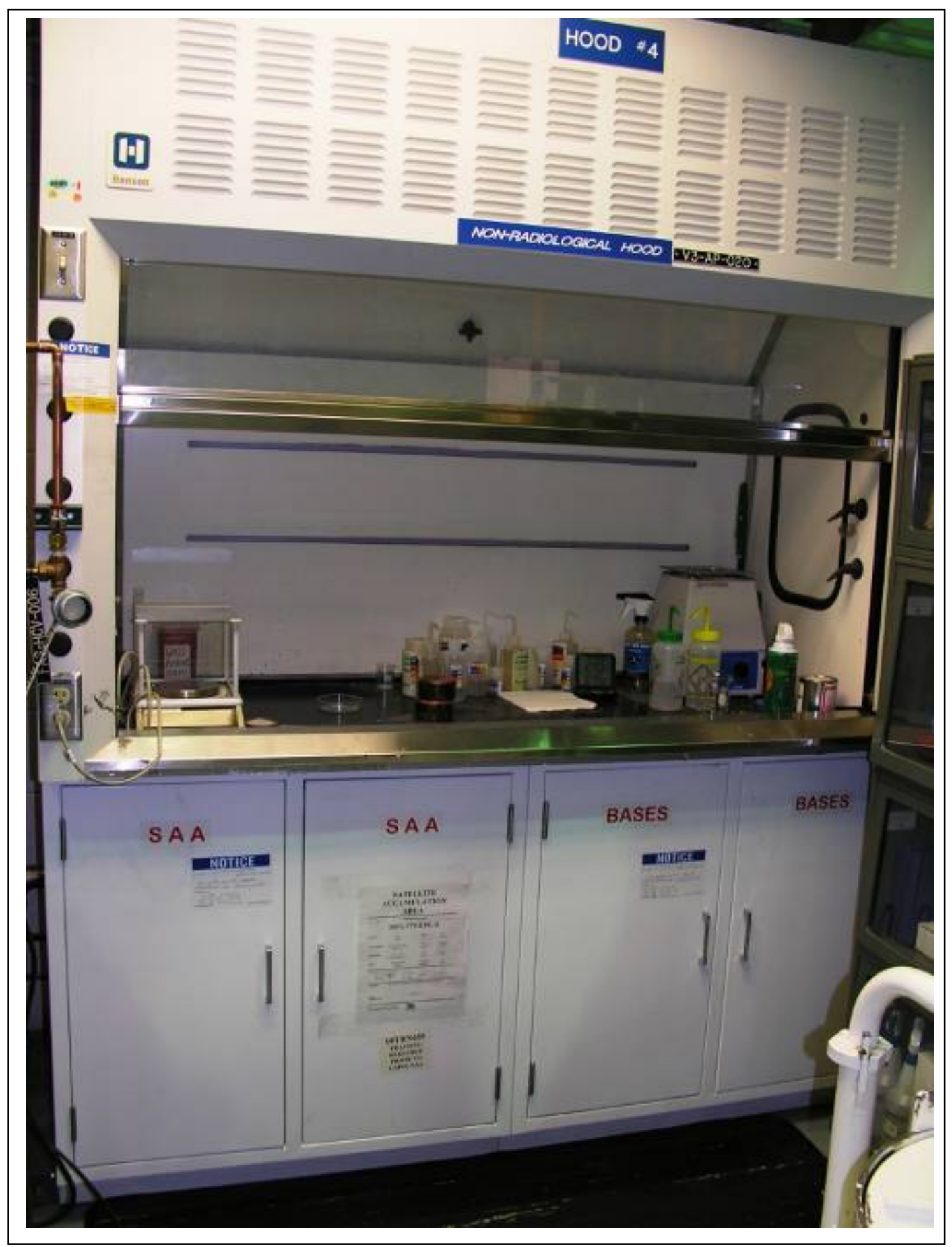

Figure B-10.a Fourth fume hood (non-radioactive) in EML, where chemical etching of samples with no nuclear material is performed. 
APPENDIX B - Photographs of Sample Preparation \& Examination Facilities

\section{B-10 EML Sample Etching Stations (continued)}

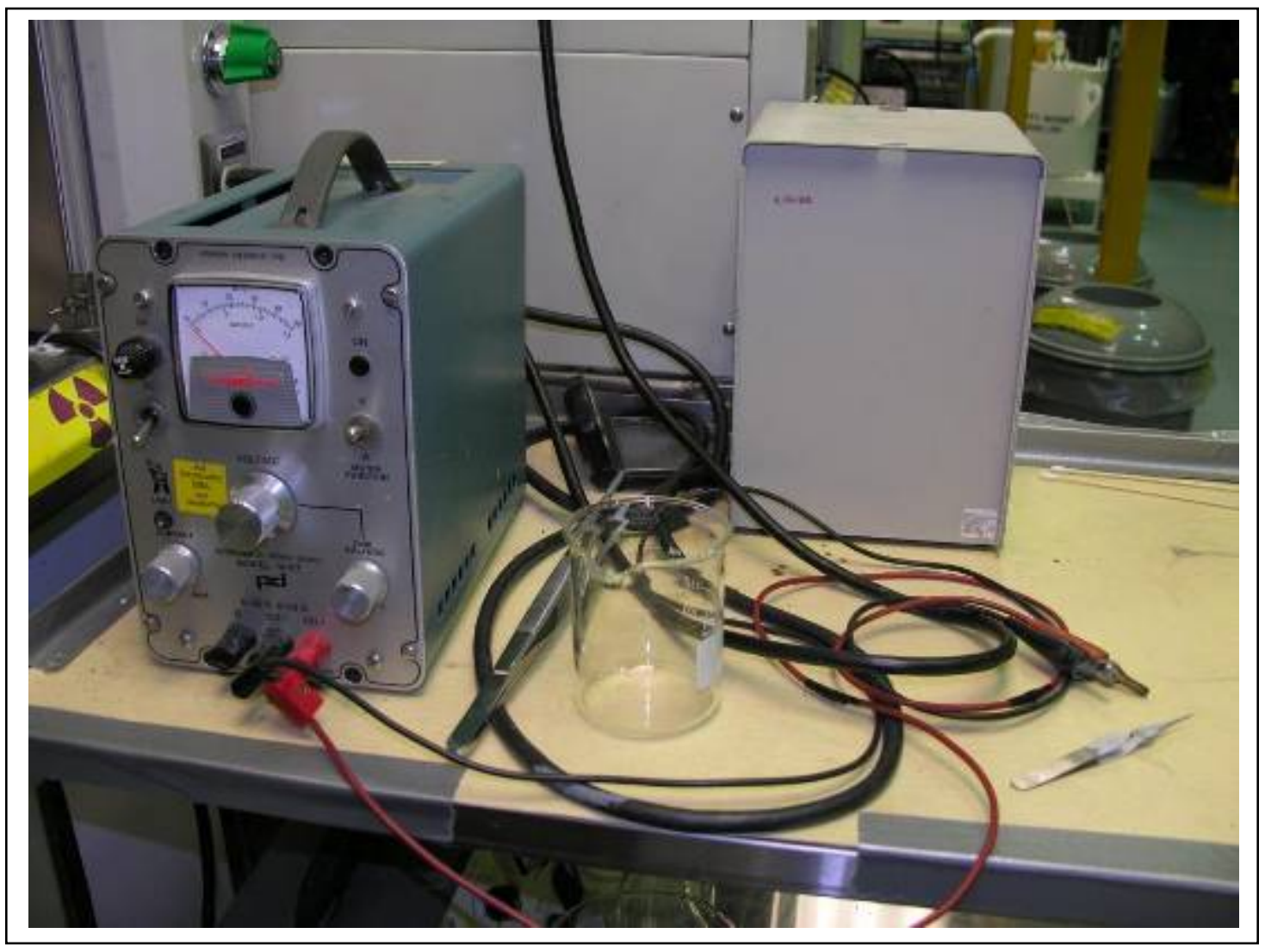

Figure B-10.b Table in front of first radiation fume hood in EML; electrochemical etching of samples containing nuclear material is performed here, where visibility is good and hood ventilation will pull air into the hood. 
APPENDIX B - Photographs of Sample Preparation \& Examination Facilities

\section{B-11 EML Computer, Optical Microscopes, and Microhardness Tester}

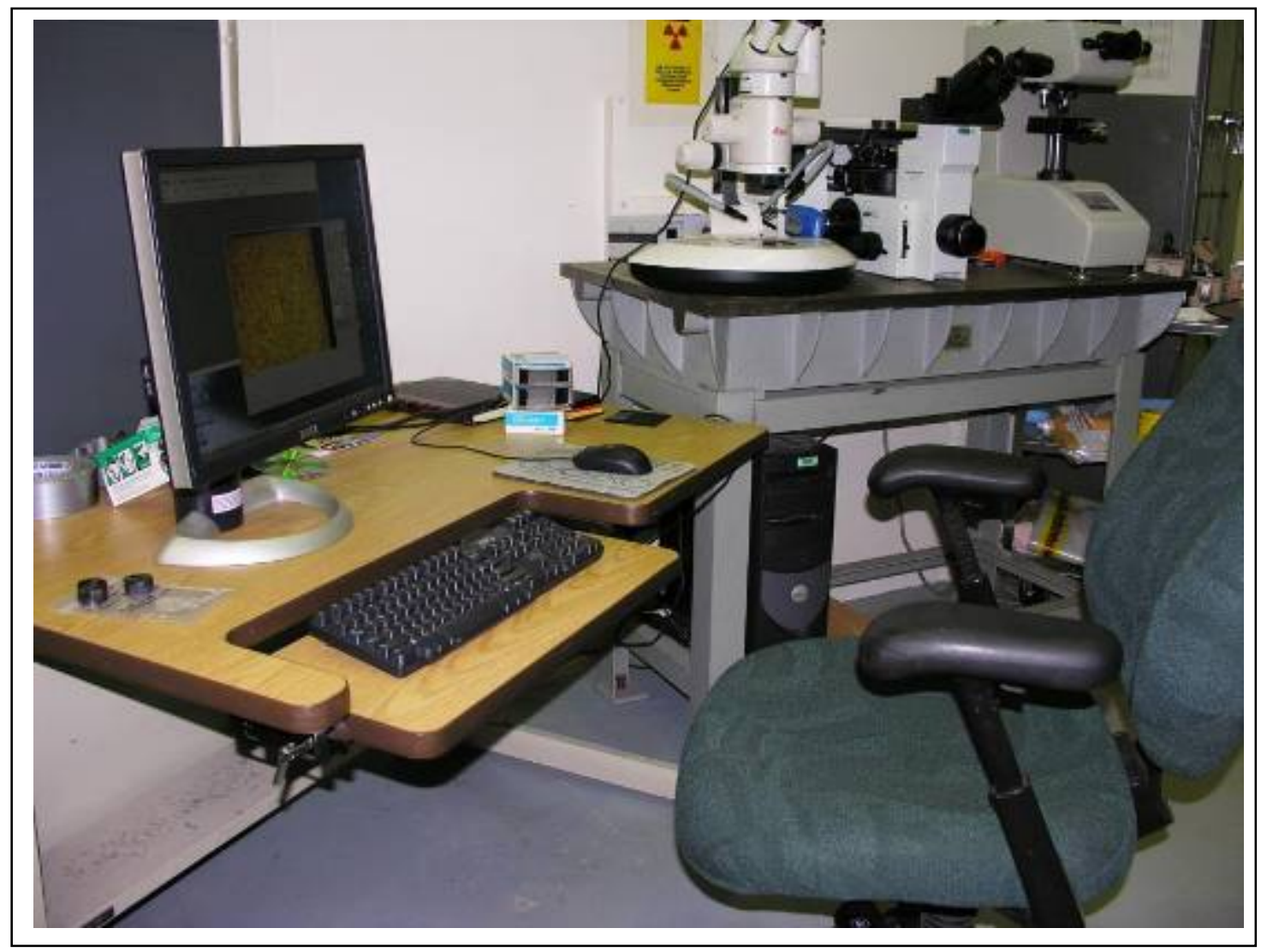

Figure B-11.a EML computer, optical microscopes, and microhardness tester. 
APPENDIX B - Photographs of Sample Preparation \& Examination Facilities

B-11 EML Computer, Optical Microscopes, and Microhardness Tester (continued)

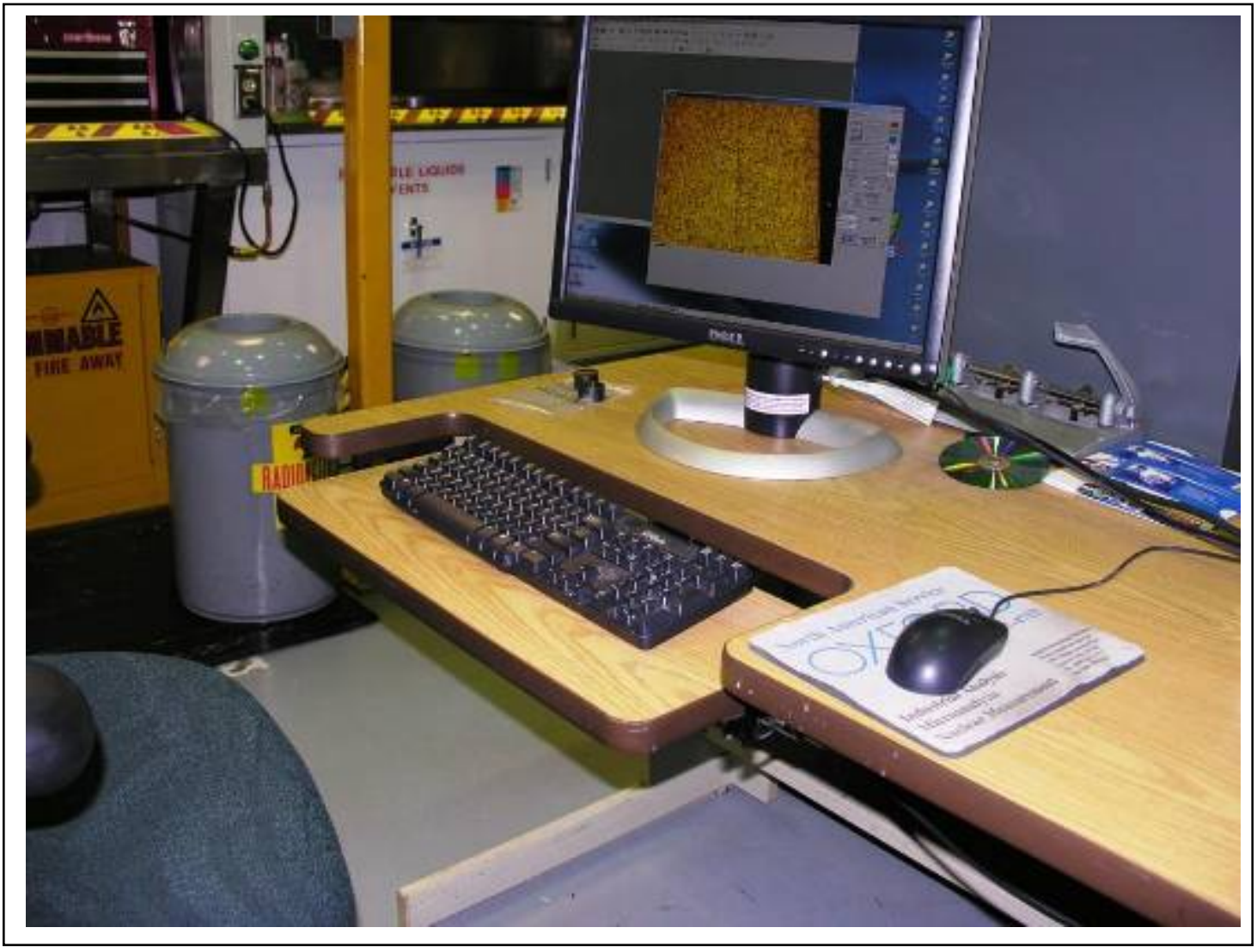

Figure B-11.b EML Dell PC computer. 
APPENDIX B - Photographs of Sample Preparation \& Examination Facilities

B-11 EML Computer, Optical Microscopes, and Microhardness Tester (continued)

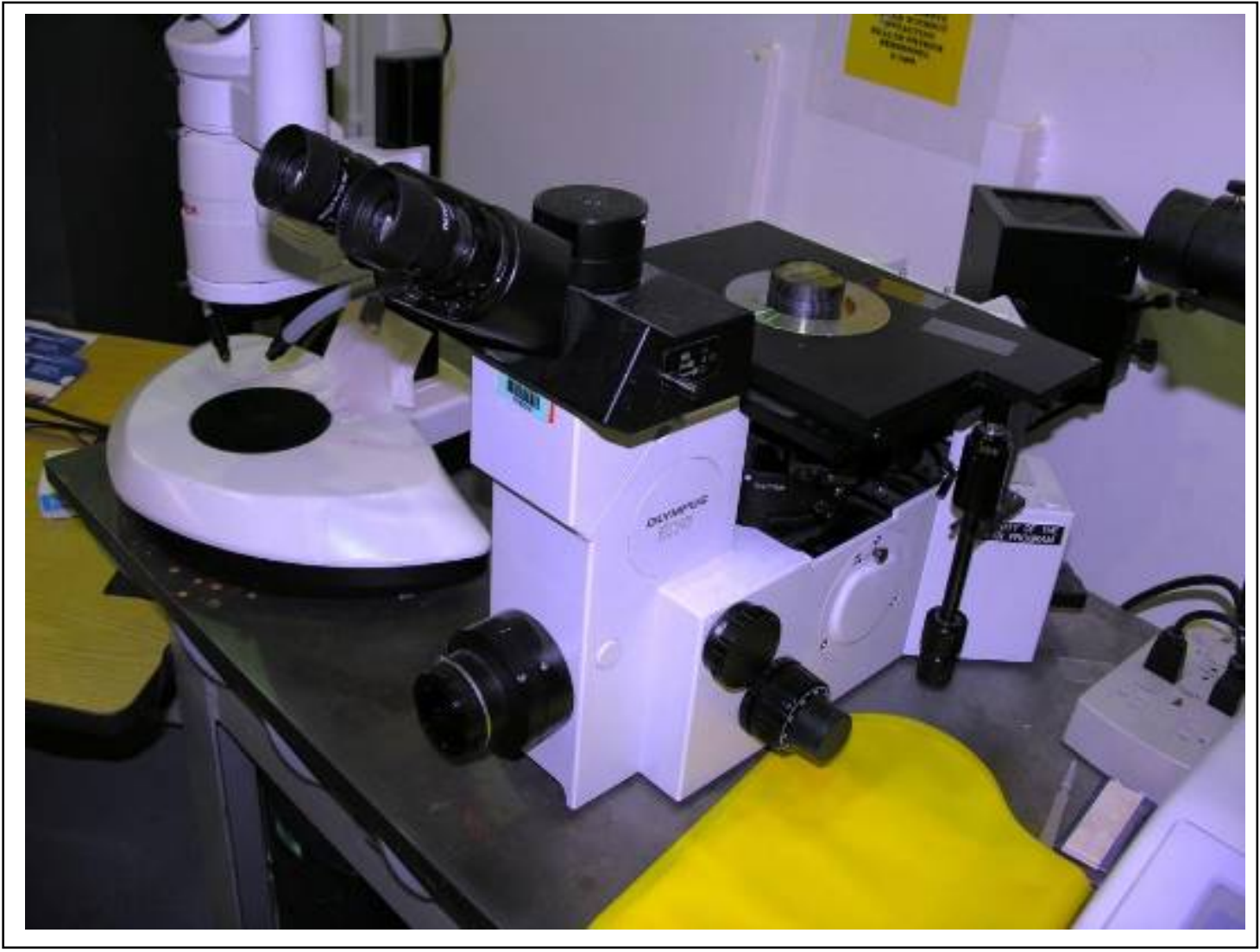

Figure B-11.c EML optical microscope (Olympus IX70), with sample ready for viewing. 
APPENDIX B - Photographs of Sample Preparation \& Examination Facilities

B-11 EML Computer, Optical Microscopes, and Microhardness Tester (continued)

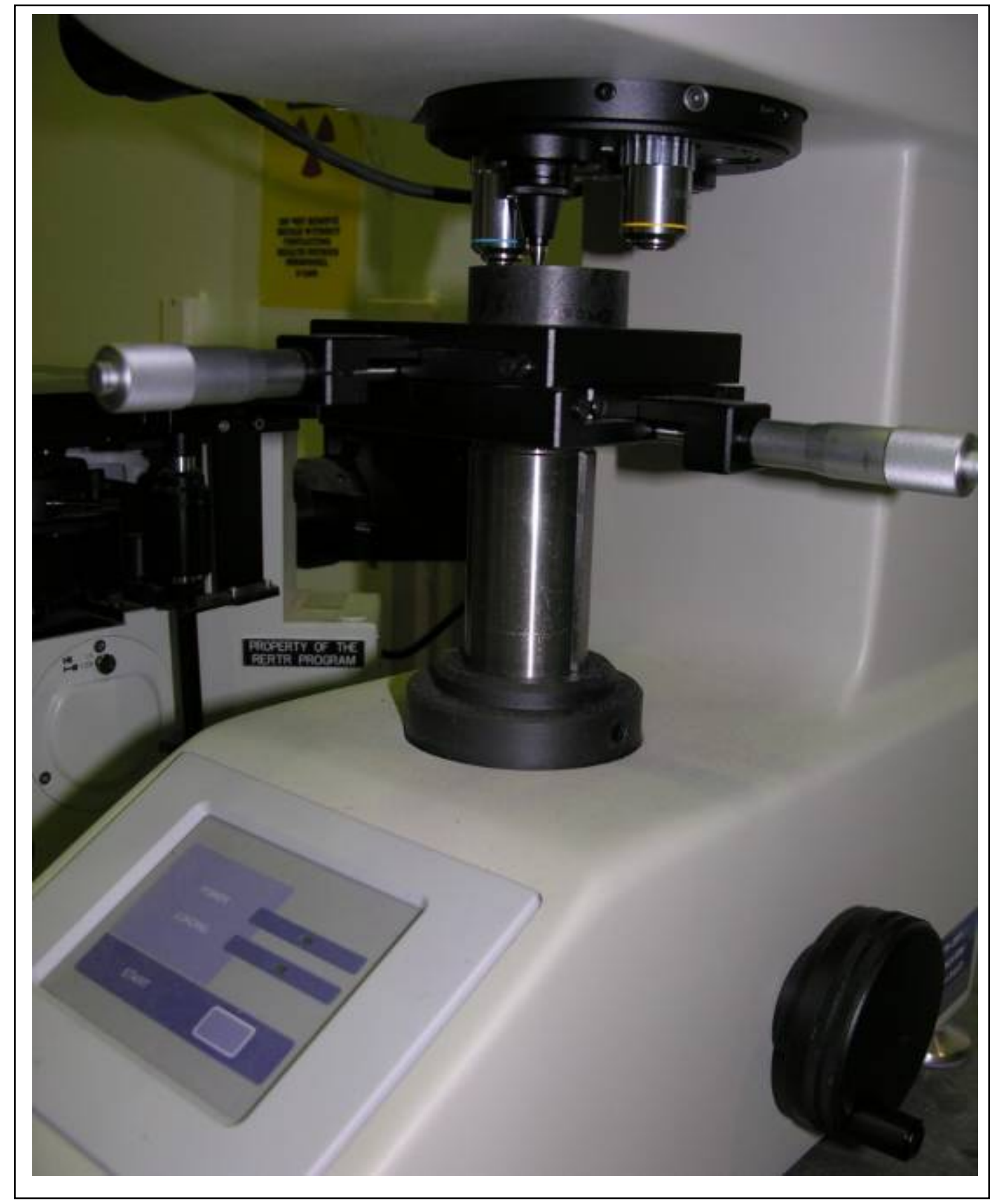

Figure B-11.d EML microhardness tester (LECO Corp Model LM-100) with sample being tested. 
APPENDIX B - Photographs of Sample Preparation \& Examination Facilities

(page is intentionally blank) 


\title{
Microstructural Examination to Aid in Understanding \\ Friction Bonding Fabrication Technique \\ for Monolithic Nuclear Fuel
}

\begin{abstract}
APPENDIX C
Microphotos of One-Sided, Single-Pass Friction Bonded Aluminum Samples

(See Figure 5.3 in main report text for microphoto locations on sample)

Figure

C-1 Microphotos of Sample Fabricated with 35.6 kN (8,000 lbf) Target FB Tool Load

C-2 Microphotos of Sample Fabricated with 44.5 kN (10,000 lbf) Target FB Tool Load

C-3 Microphotos of Sample Fabricated with 53.4 kN (12,000 lbf) Target FB Tool Load

C-4 Microphotos of Sample Fabricated with 62.3 kN (14,000 lbf) Target FB Tool Load
\end{abstract}




\section{APPENDIX C}

Microphotos of One-Sided, Single-Pass Friction Bonded Aluminum Samples

(page is intentionally blank) 


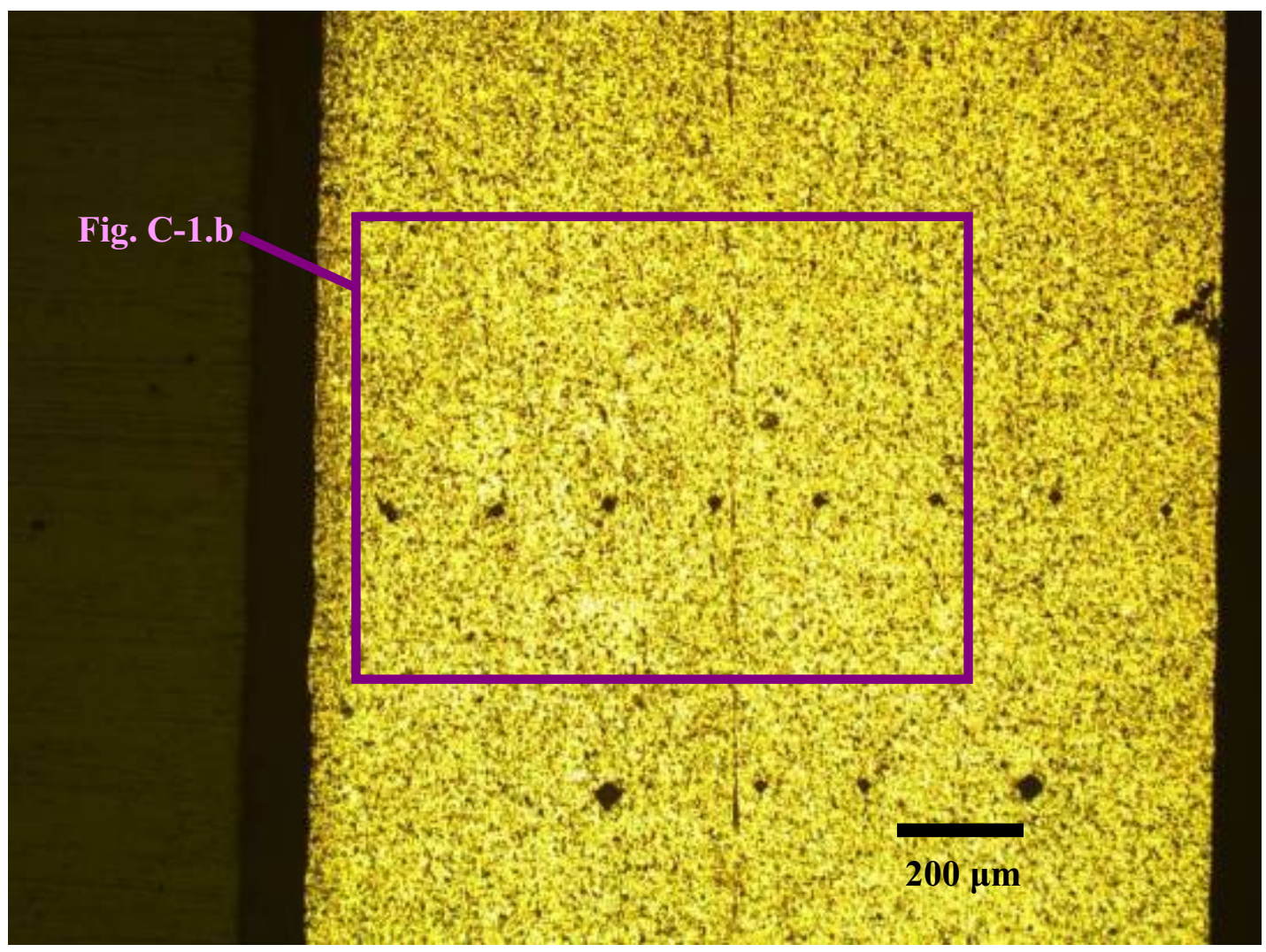

Figure C-1.a "Center" location on etched aluminum sample with 1-sided, 1-pass FB, fabricated with FB tool load of $35.6 \mathrm{kN}(8,000 \mathrm{lbf}), 50 \mathrm{X}$. 


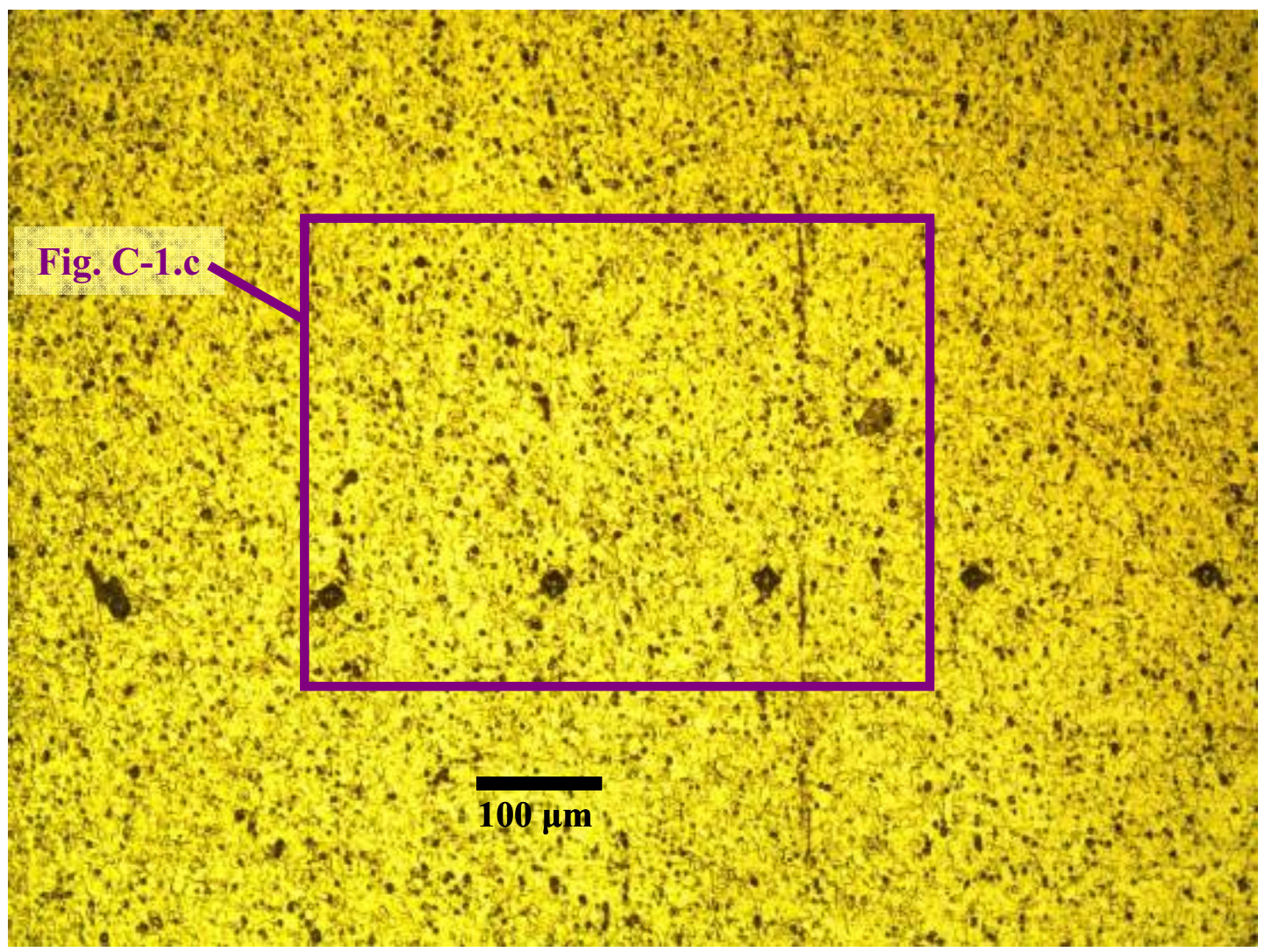

Figure C-1.b "Center" location on etched aluminum sample with 1-sided, 1-pass FB, fabricated with FB tool load of $35.6 \mathrm{kN}$ (8,000 lbf), 100X.

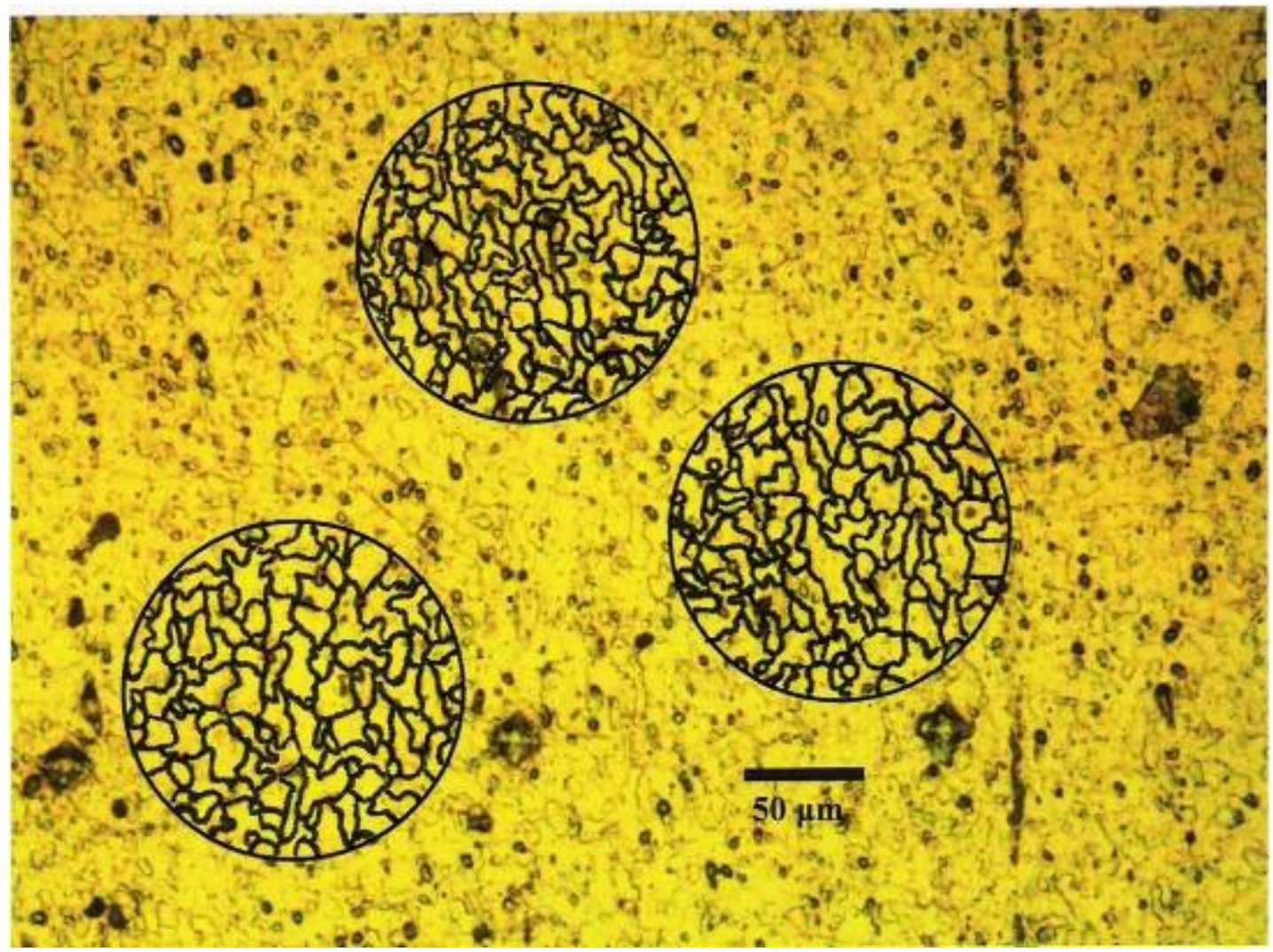

Figure C-1.c "Center" location on etched aluminum sample, FB tool load of $35.6 \mathrm{kN}$ (8,000 lbf), 200X, grain size measurements. Avg. for 3 circles: 79 grains within circle $+\left(33^{2} / 3\right.$ grains cut by circumf. $) / 2=$ $95^{5} / 6$ grains. So $\mathrm{N}=95^{5} / 6$ grains $/ 0.25 \mathrm{in}^{2}=383^{1} / 3$ grains $/ 1 \mathrm{in}^{2}=2^{(\mathrm{G}-1)} \rightarrow \mathrm{G}=\ln 383^{1} / 3 / \ln 2+1=9.6$. 


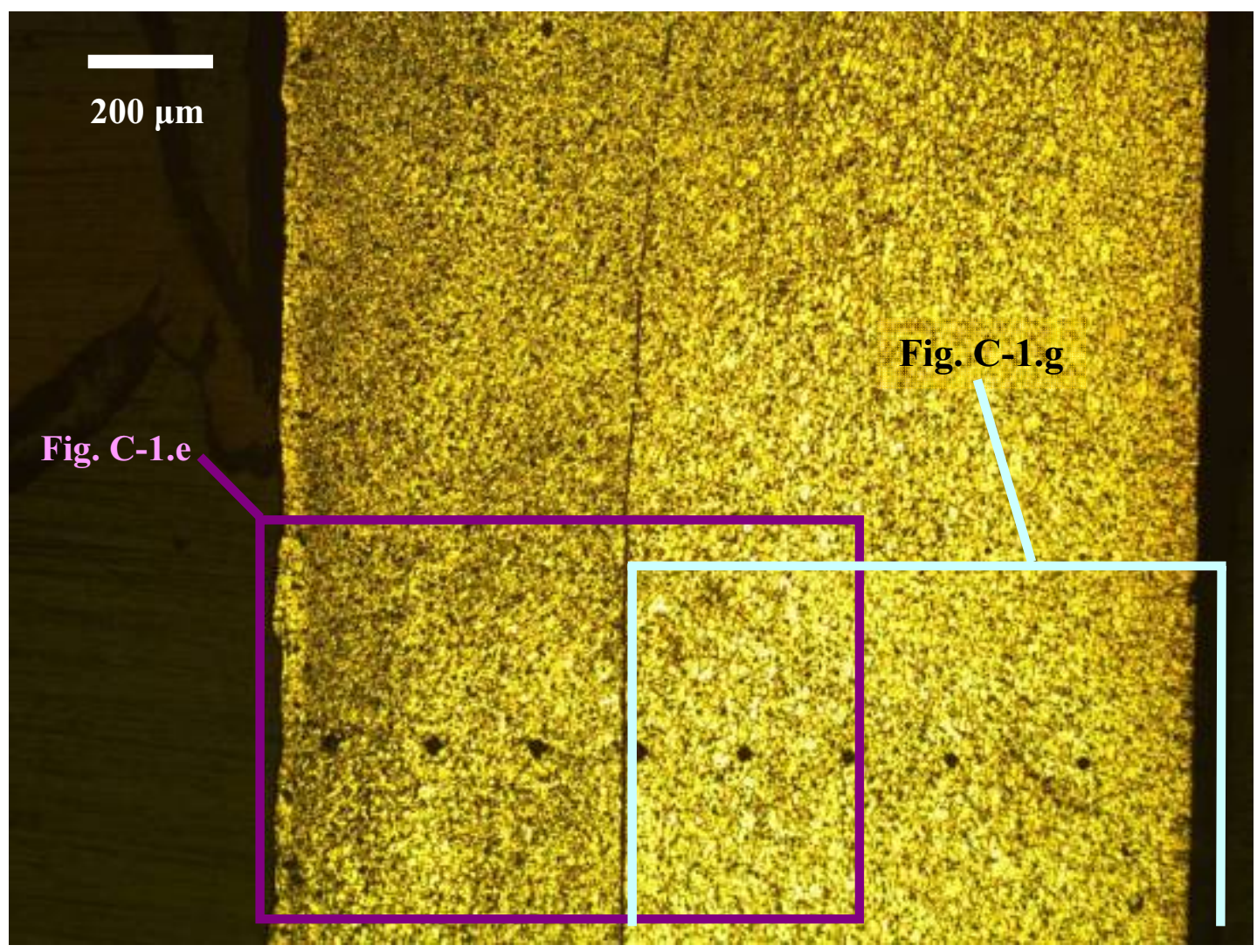

Figure C-1.d "Retreating edge" location on etched aluminum sample with 1-sided, 1-pass FB, fabricated with FB tool load of $35.6 \mathrm{kN}(8,000 \mathrm{lbf}), 50 \mathrm{X}$.

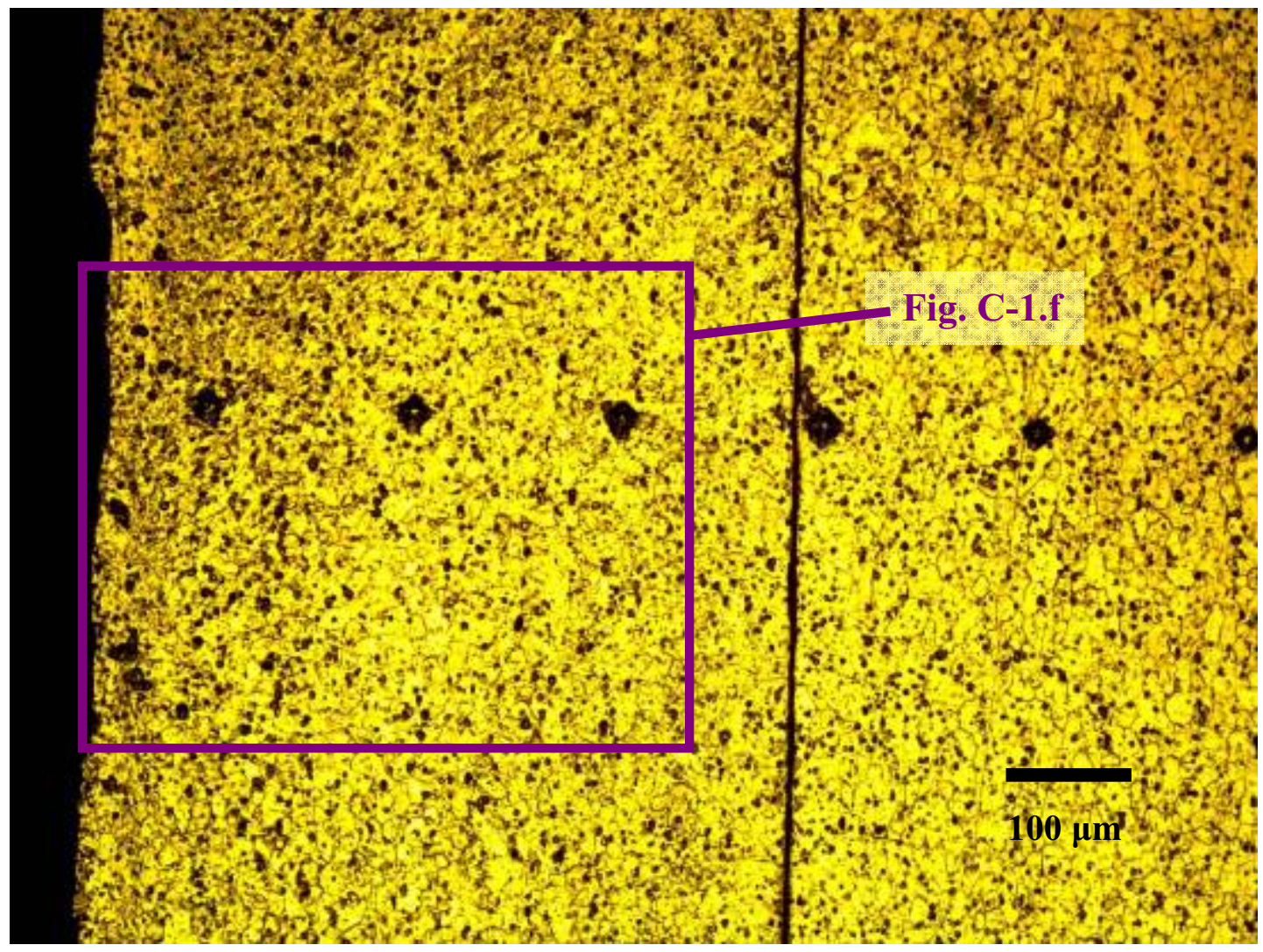

Figure C-1.e FB side of "retreating edge" location on etched aluminum sample with 1-sided, 1-pass FB, fabricated with FB tool load of $35.6 \mathrm{kN}$ (8,000 lbf), 100X. 


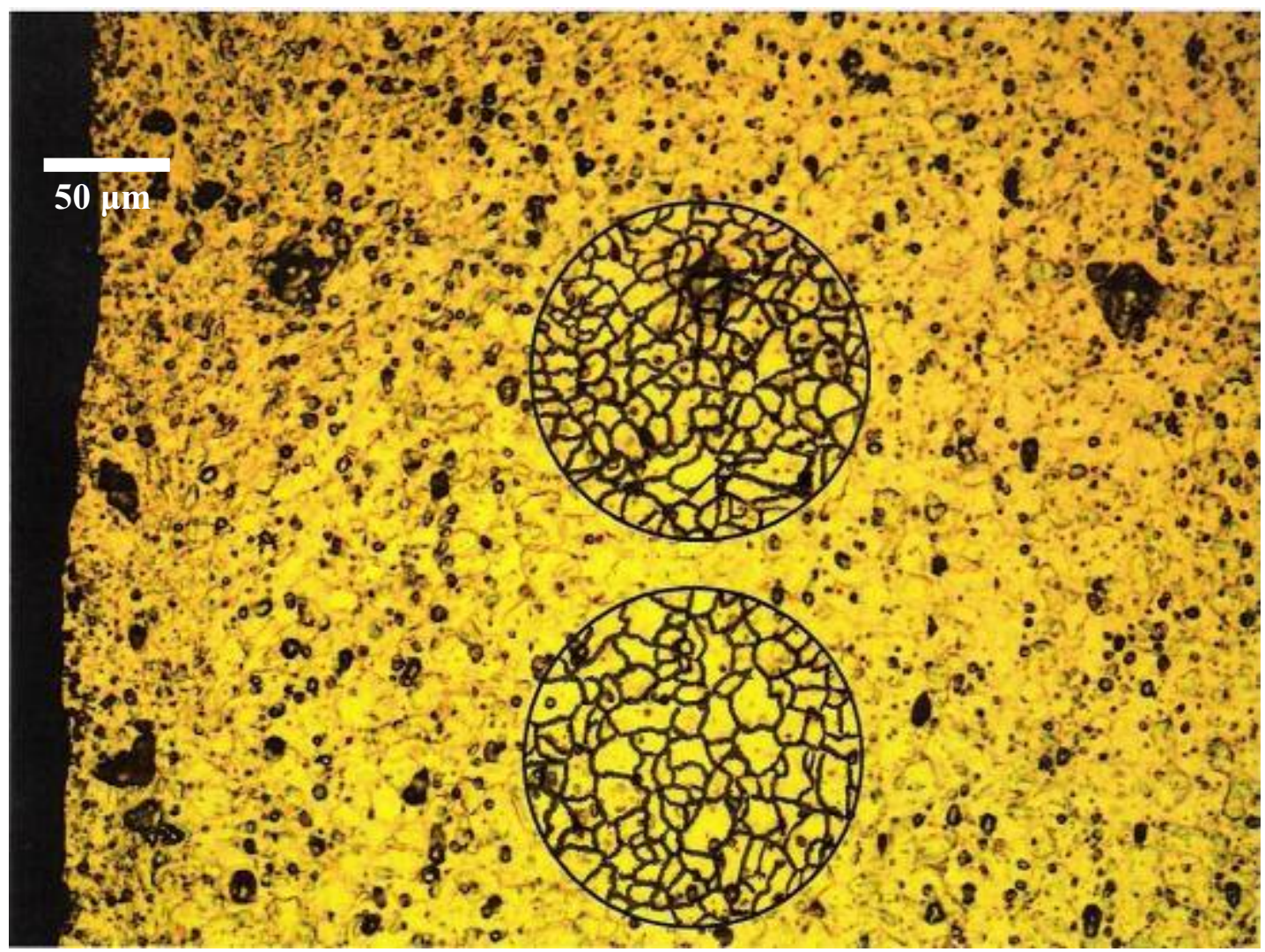

Figure C-1.f FB side of "retreating edge" location on etched aluminum sample, FB tool load of 35.6 kN (8,000 lbf), 200X, grain size measurements. Avg. for 2 circles: 104 grains within circle $+\left(38^{1} / 2\right.$ grains cut by ircumf. $) / 2=123^{1} / 4$ grains. So $\mathrm{N}=123 \frac{1}{4}$ grains $/ 0.25 \mathrm{in}^{2}=493$ grains $/ \operatorname{lin}^{2}=2^{(\mathrm{G}-1)} \rightarrow \mathrm{G}=\ln 493 / \ln 2+1=9.9$

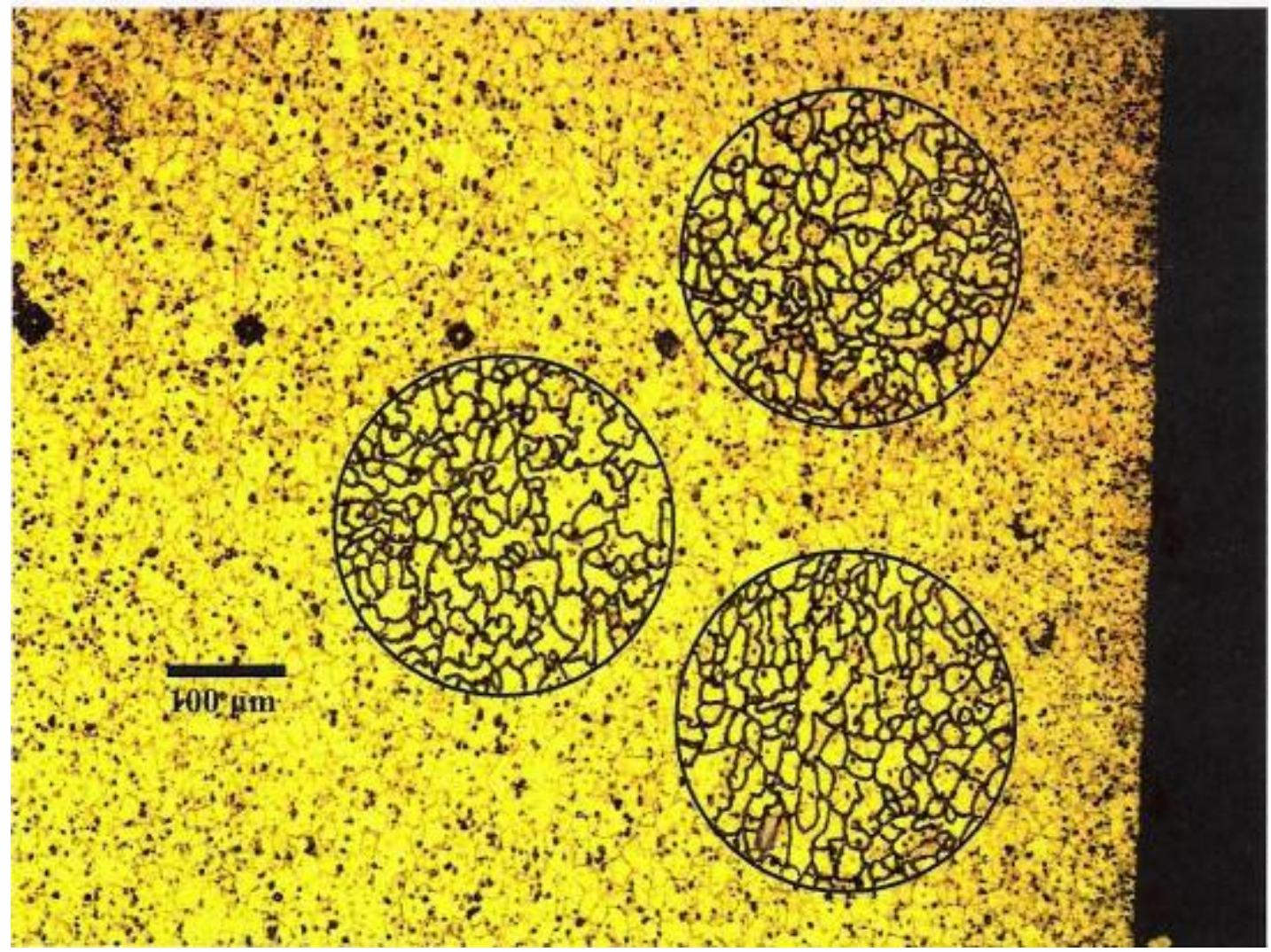

Figure C-1.g Non-FB side of "retreating edge" location on etched aluminum sample, FB tool load of 35.6 $\mathrm{kN}(8,000 \mathrm{lbf}), 100 \mathrm{X}$, grain size measurements. Avg. for 3 circles: 121 grains within circle $+\left(43^{2} / 3\right.$ grains cut by circumf.) $/ 2=142^{5} / 6$ grains. So $\mathrm{N}=142^{5} / 6$ grains $/ 1$ in $^{2}=2^{(\mathrm{G}-1)} \rightarrow \mathrm{G}=\ln 142^{5} / 6 / \ln 2+1=8.2$. 


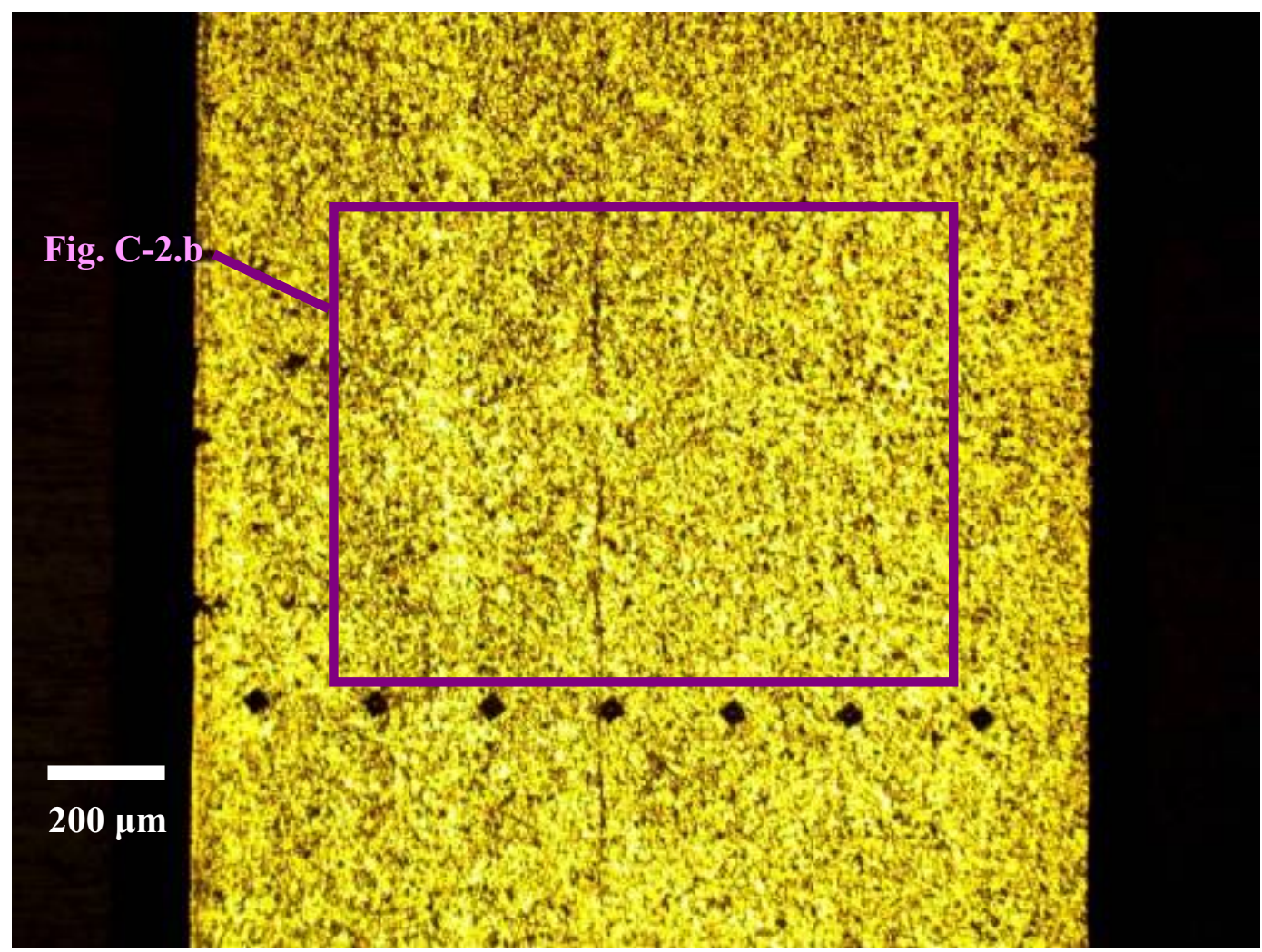

Figure C-2.a "Center" location on etched aluminum sample with 1-sided, 1-pass FB fabricated with FB tool load of $44.5 \mathrm{kN}(10,000 \mathrm{lbf}), 50 \mathrm{X}$.

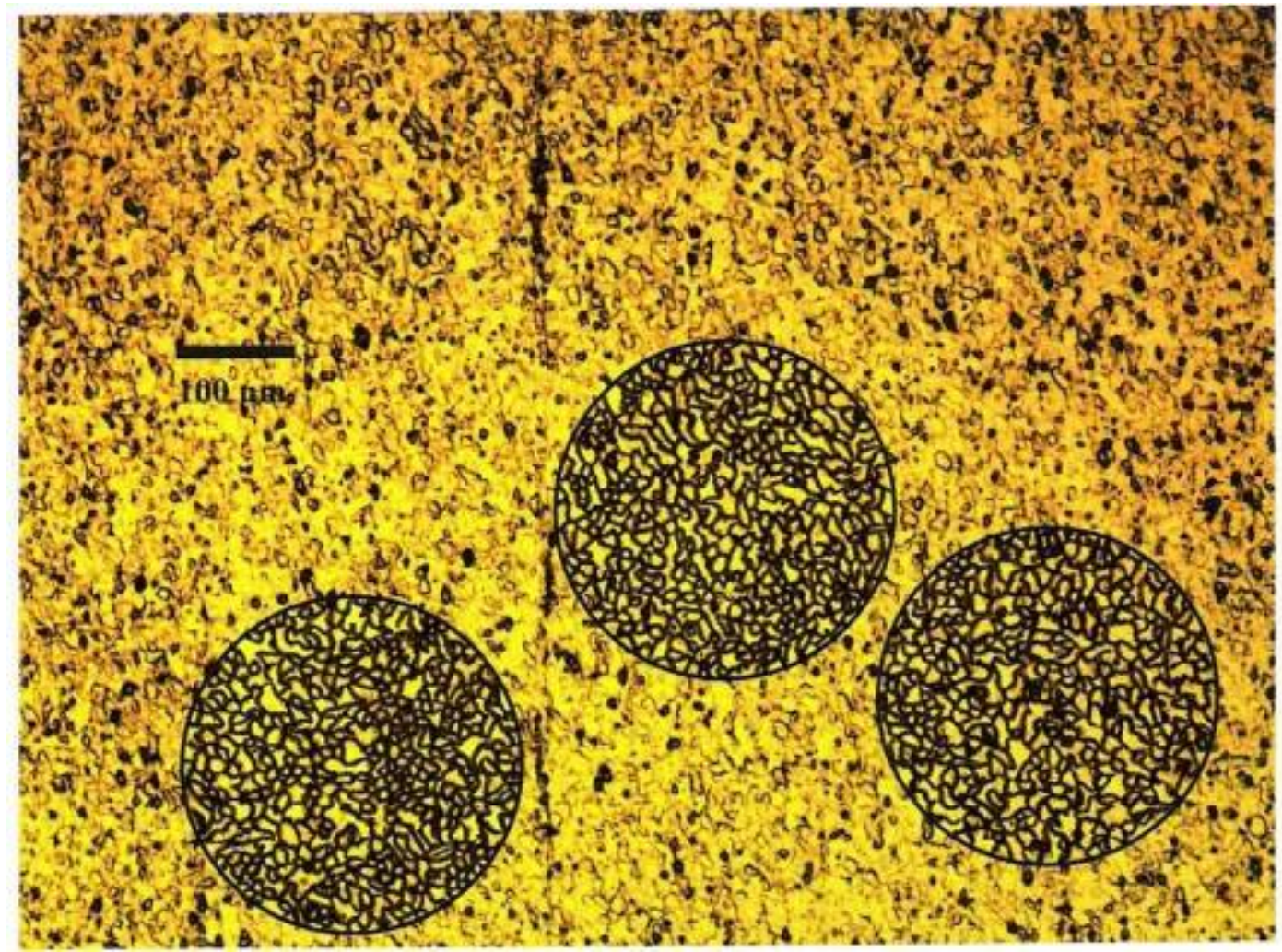

Figure C-2.b "Center" location on etched aluminum sample, FB tool load of $44.5 \mathrm{kN}$ (10,000 lbf), $100 \mathrm{X}$, grain size measurements. Avg. for 3 circles: $338^{2} / 3$ grains within circle $+(61$ grains cut by circumf. $) / 2=369^{1} / 6$ grains. So $\mathrm{N}=369^{1} / 6$ grains $/ 1 \operatorname{in}^{2}=2^{(\mathrm{G}-1)} \rightarrow \mathrm{G}=\ln 369^{1} / 6 / \ln 2+1=9.5$. 


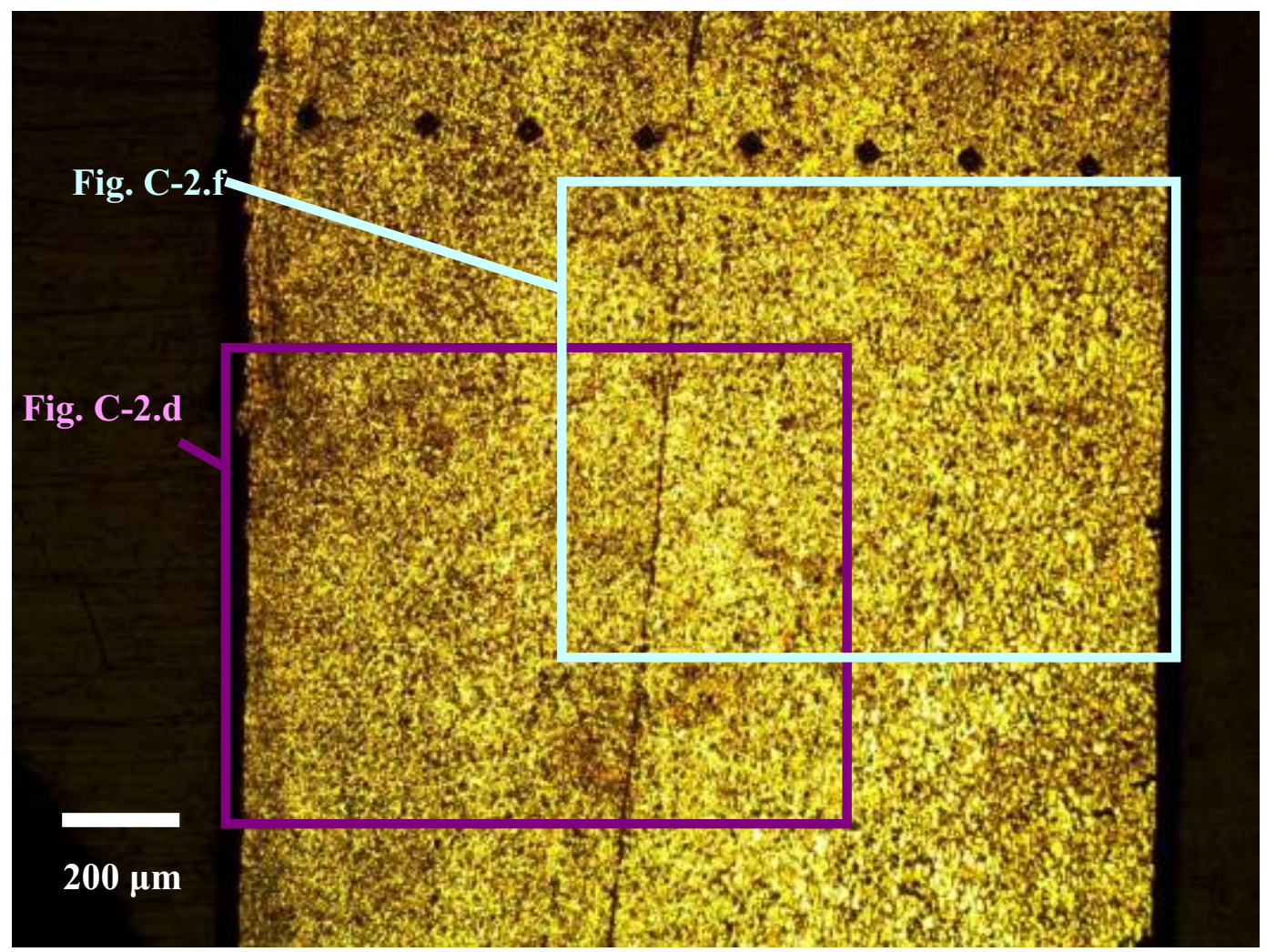

Figure C-2.c "Retreating edge" location on etched aluminum sample with 1-sided, 1-pass FB fabricated with FB tool load of $44.5 \mathrm{kN}(10,000 \mathrm{lbf}), 50 \mathrm{X}$.

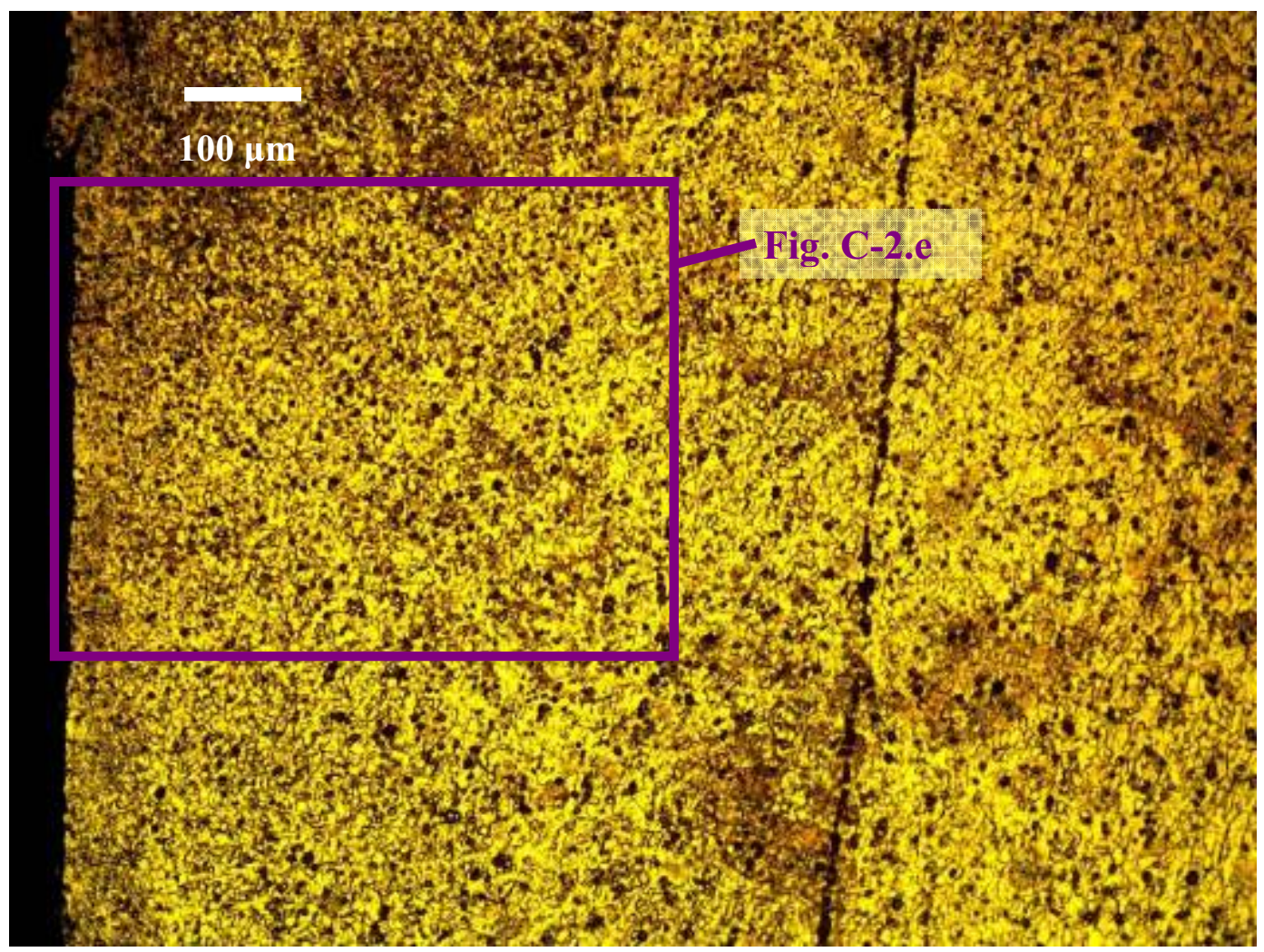

Figure C-2.d FB side of "retreating edge" location on etched aluminum sample with 1-sided, 1-pass FB fabricated with FB tool load of $44.5 \mathrm{kN}$ (10,000 lbf), 100X. 


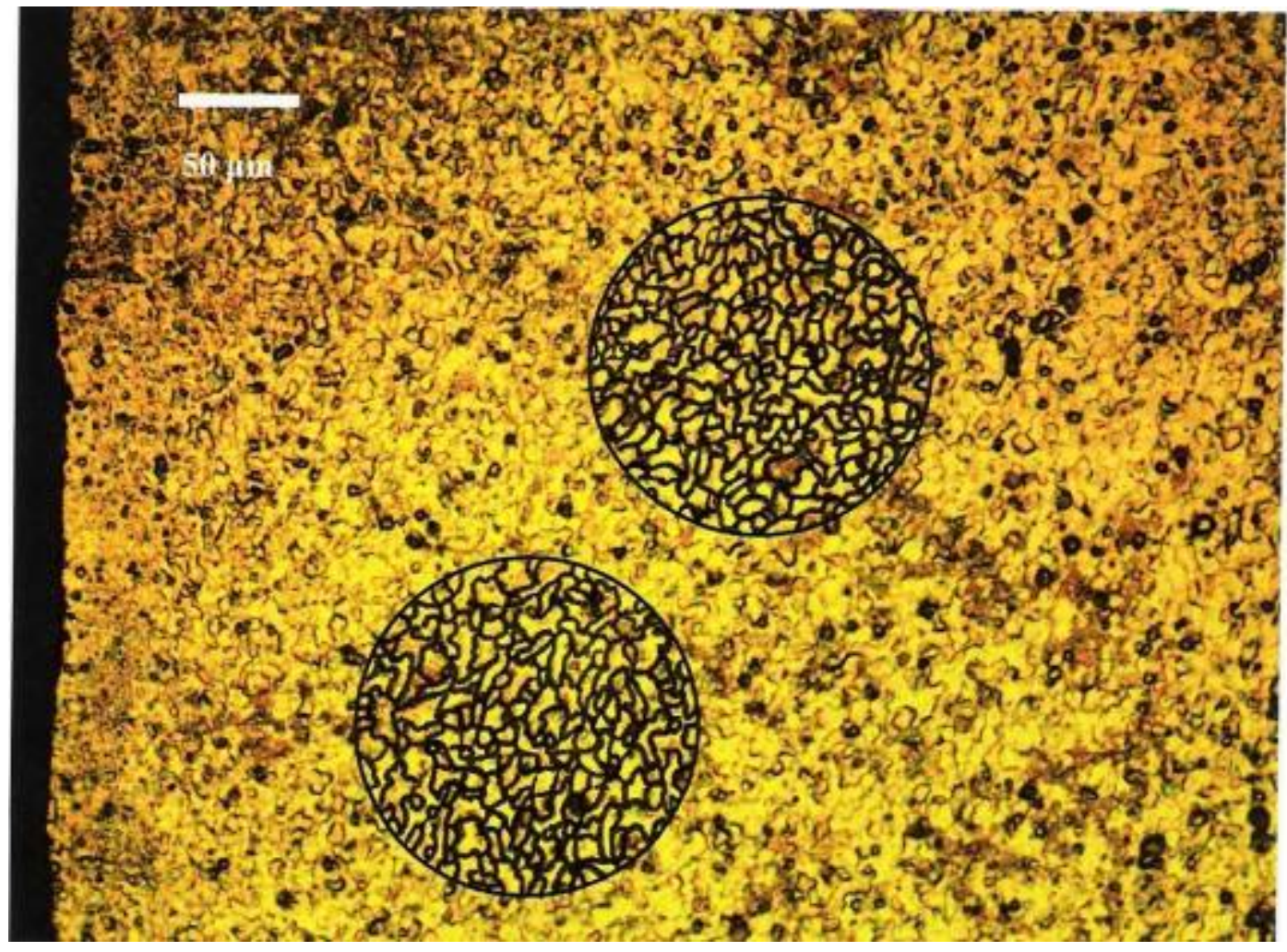

Figure C-2.e FB side of "retreating edge" location on etched aluminum sample, FB tool load of $44.5 \mathrm{kN}(10,000 \mathrm{lbf})$, 200X, grain size measurements. Avg. for 2 circles: 179 grains within circle $+(49$ grains cut by circumf. $) / 2=203^{1} / 2$ grains. So $\mathrm{N}=203 \frac{1}{2}$ grains $/ 0.25 \mathrm{in}^{2}=814$ grains $/ 1 \mathrm{in}^{2}=2^{(\mathrm{G}-1)} \rightarrow \mathrm{G}=\ln 814 / \ln 2+1=10.7$.

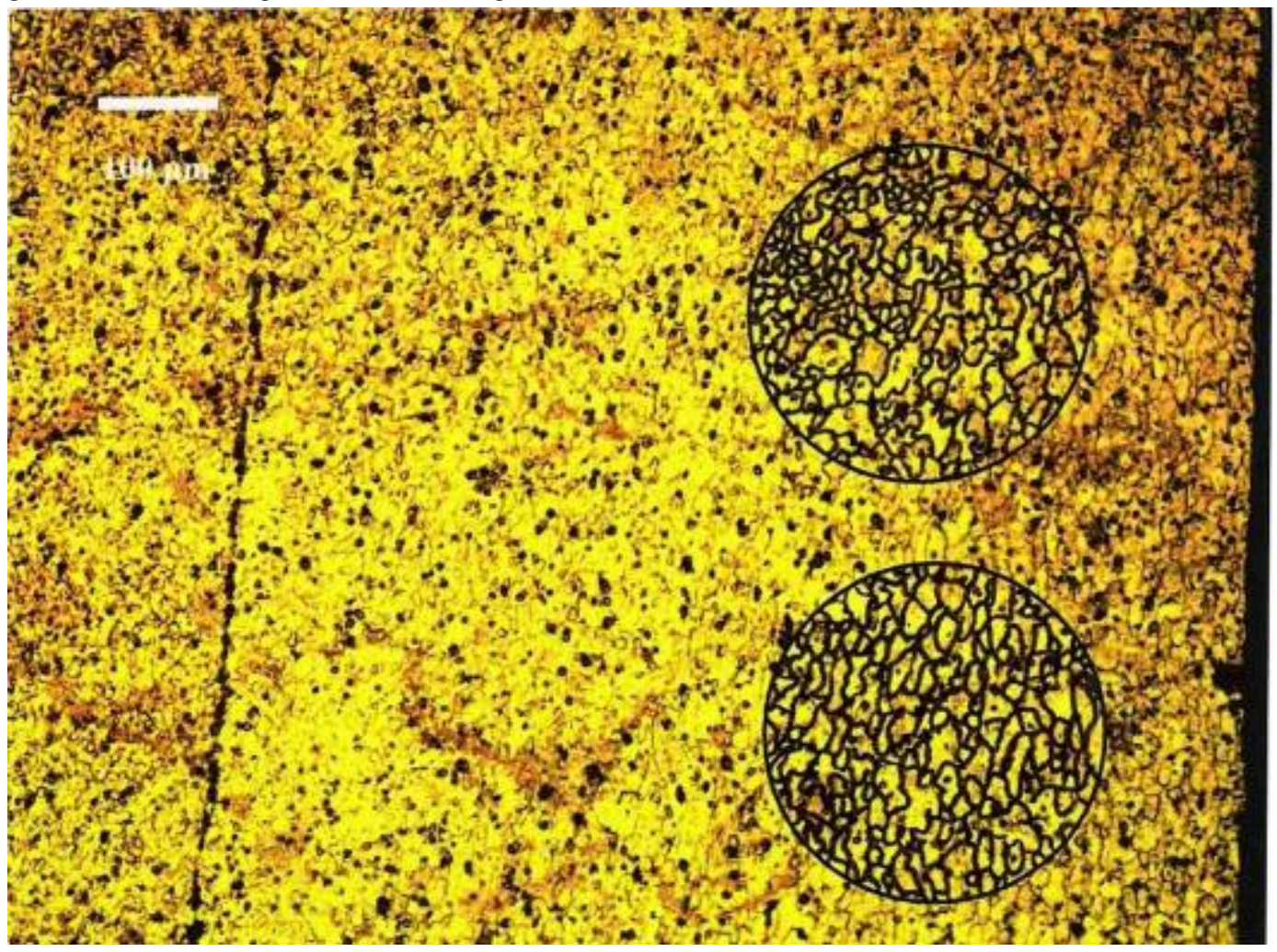

Figure C-2.f Non-FB side of "retreating edge" location on etched aluminum sample, FB tool load of 44.5 $\mathrm{kN}(10,000 \mathrm{lbf}), 100 \mathrm{X}$, grain size measurements. Avg. for 2 circles: $156^{1} / 2$ grains within circle $+\left(43^{1} / 2\right.$ grains cut by circumf. $) / 2=168^{1} / 4$ grains. So $\mathrm{N}=168^{1} / 4$ grains $/ 1 \mathrm{in}^{2}=2^{(\mathrm{G}-1)} \rightarrow \mathrm{G}=\ln 168^{1} / 4 / \ln 2+1=8.4$. 
APPENDIX C. Figure C-2 Microphotos of Sample Fabricated with 44.5 kN (10,000 lbf) Target FB Tool Load

(page is intentionally blank) 


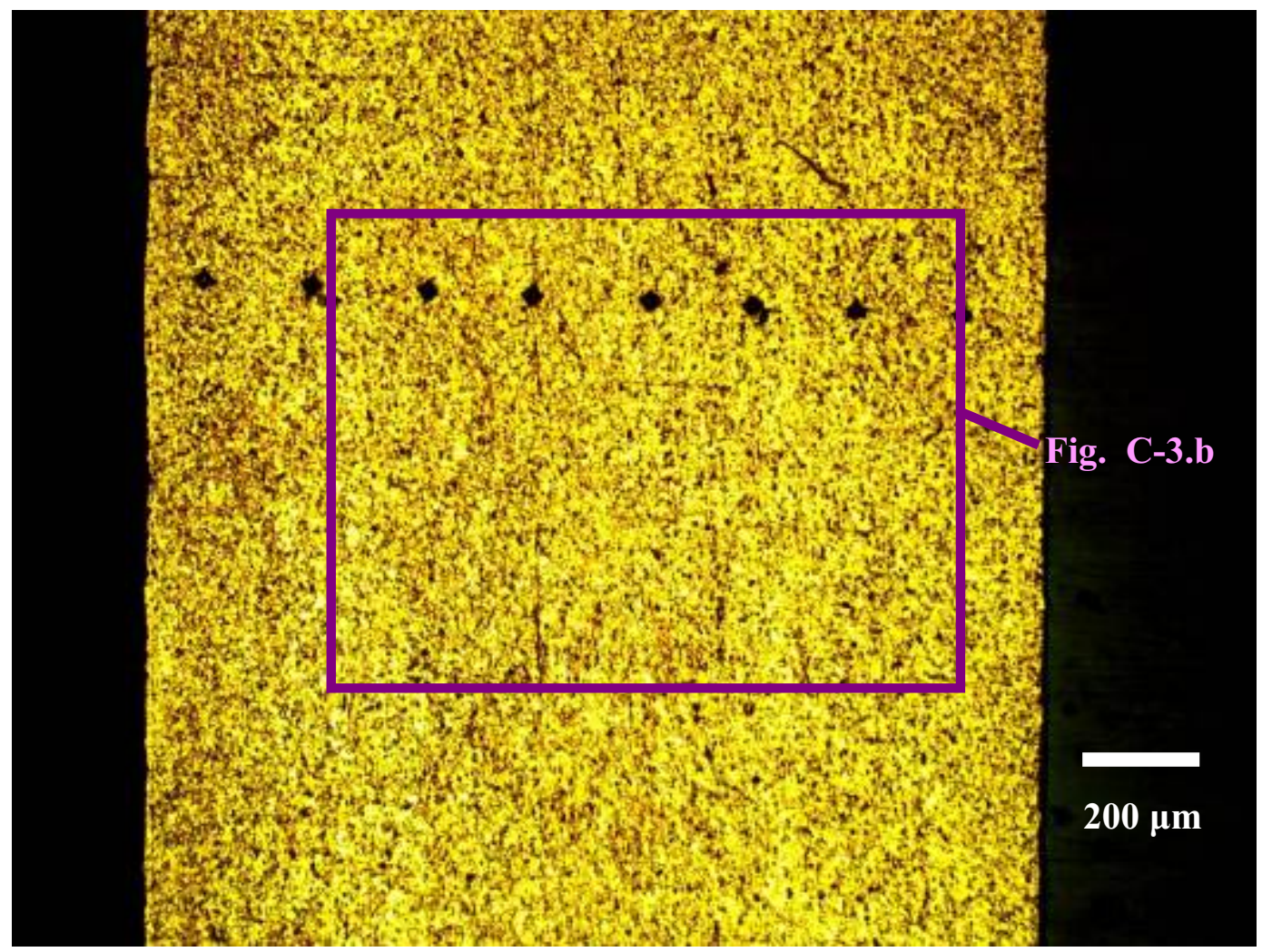

Figure C-3.a "Center" location on etched aluminum sample with 1-sided, 1-pass FB fabricated with FB tool load of $53.4 \mathrm{kN}(12,000 \mathrm{lbf}), 50 \mathrm{X}$.

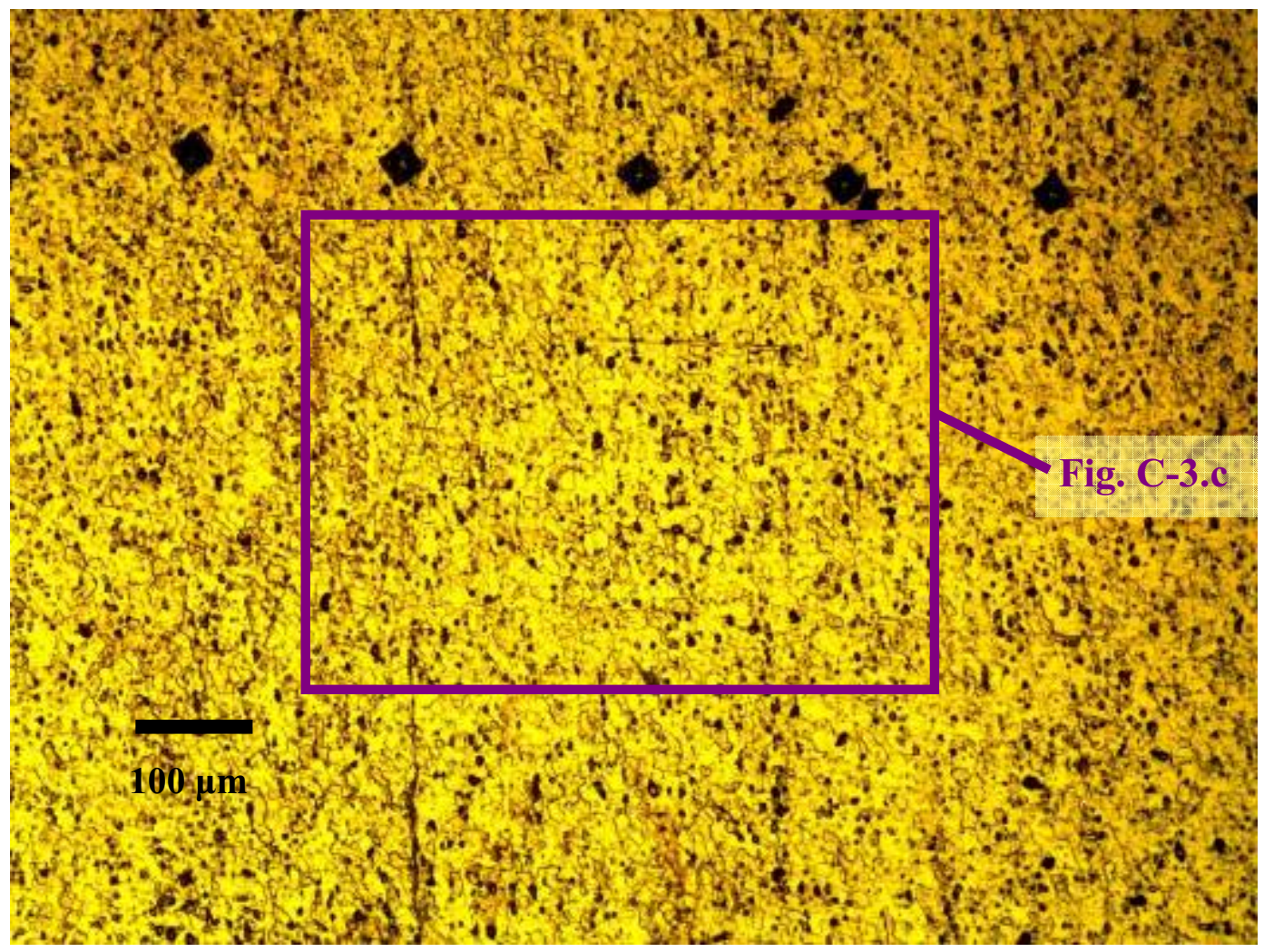

Figure C-3.b "Center" location on etched aluminum sample with 1-sided, 1-pass FB fabricated with FB tool load of $53.4 \mathrm{kN}(12,000 \mathrm{lbf}), 100 \mathrm{X}$. 


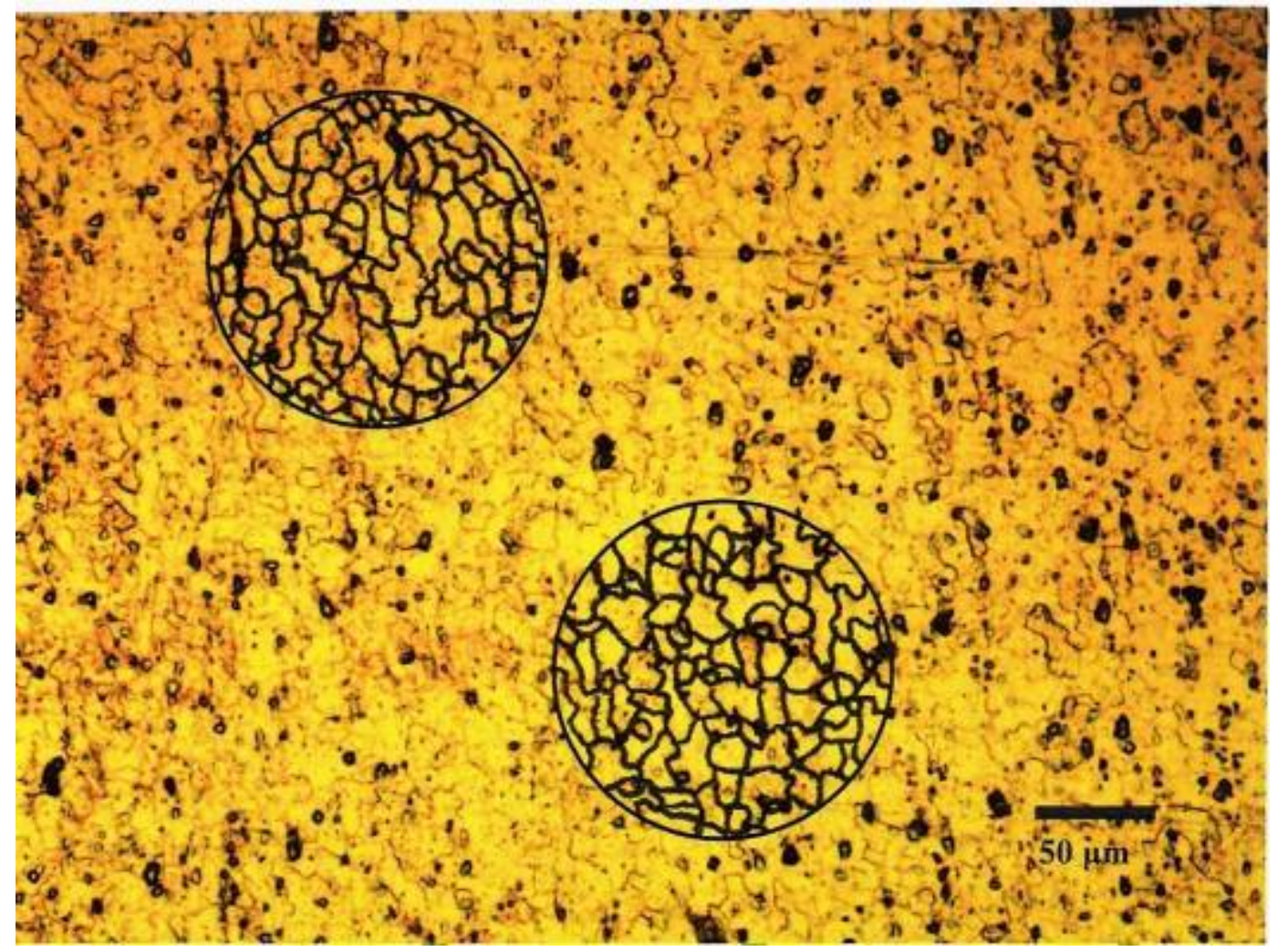

Figure C-3.c FB side of "center" location on etched aluminum sample, FB tool load of $53.4 \mathrm{kN}$ $(12,000 \mathrm{lbf}), 200 \mathrm{X}$, grain size measurements. Avg. for 2 circles: $70^{1} / 2$ grains within circle $+(281 / 2$ grains cut by circumf. $) / 2=84^{3} / 4$ grains. So $\mathrm{N}=84^{3} / 4$ grains $/ 0.25 \mathrm{in}^{2}=339$ grains $/ 1 \mathrm{in}^{2}=2^{(\mathrm{G}-1)} \rightarrow$ $\mathrm{G}=\ln 339 / \ln 2+1=9.4$.

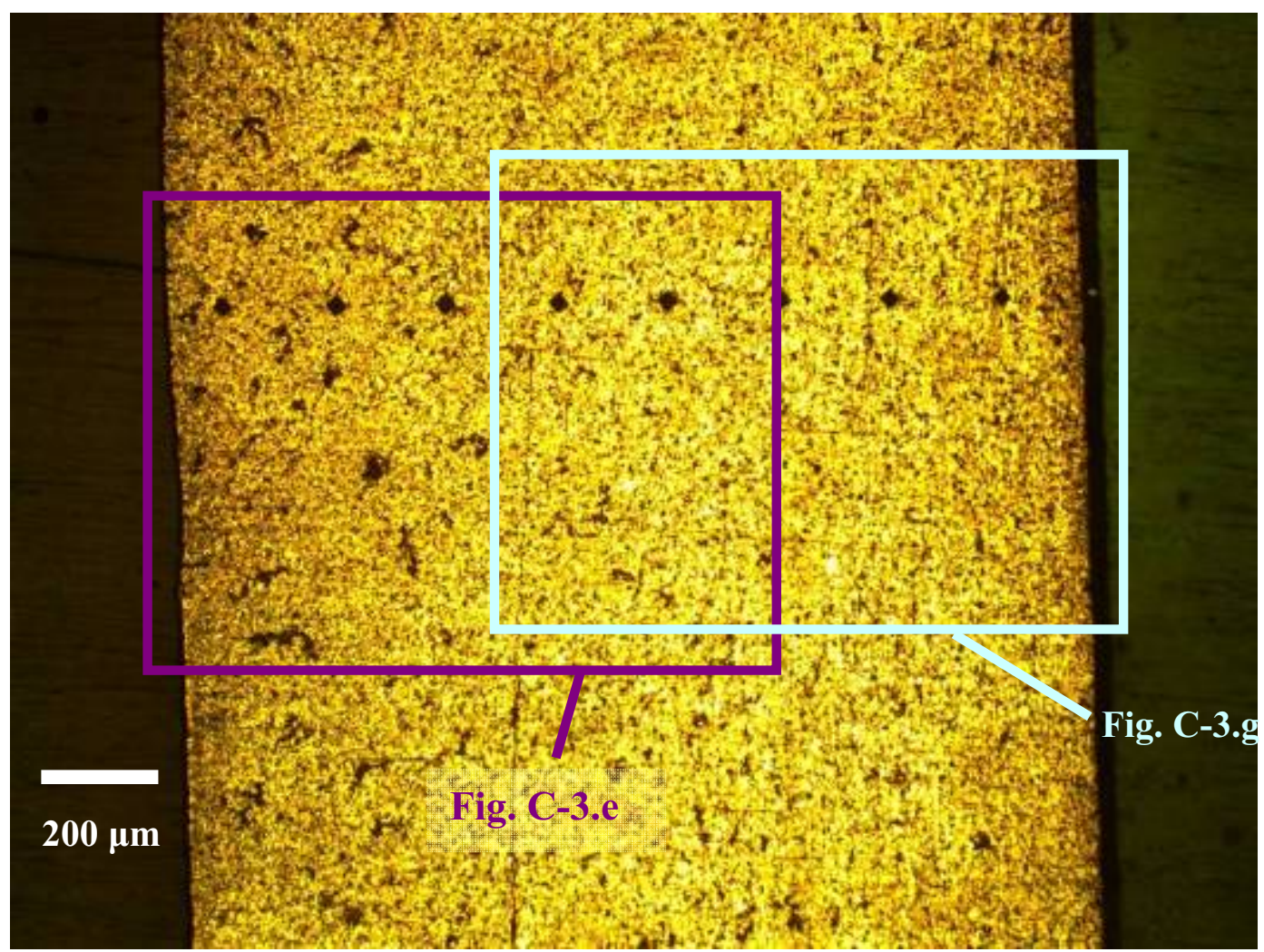

Figure C-3.d "Retreating edge" location on etched aluminum sample with 1-sided, 1-pass FB fabricated with FB tool load of $53.4 \mathrm{kN}(12,000 \mathrm{lbf}), 50 \mathrm{X}$. 


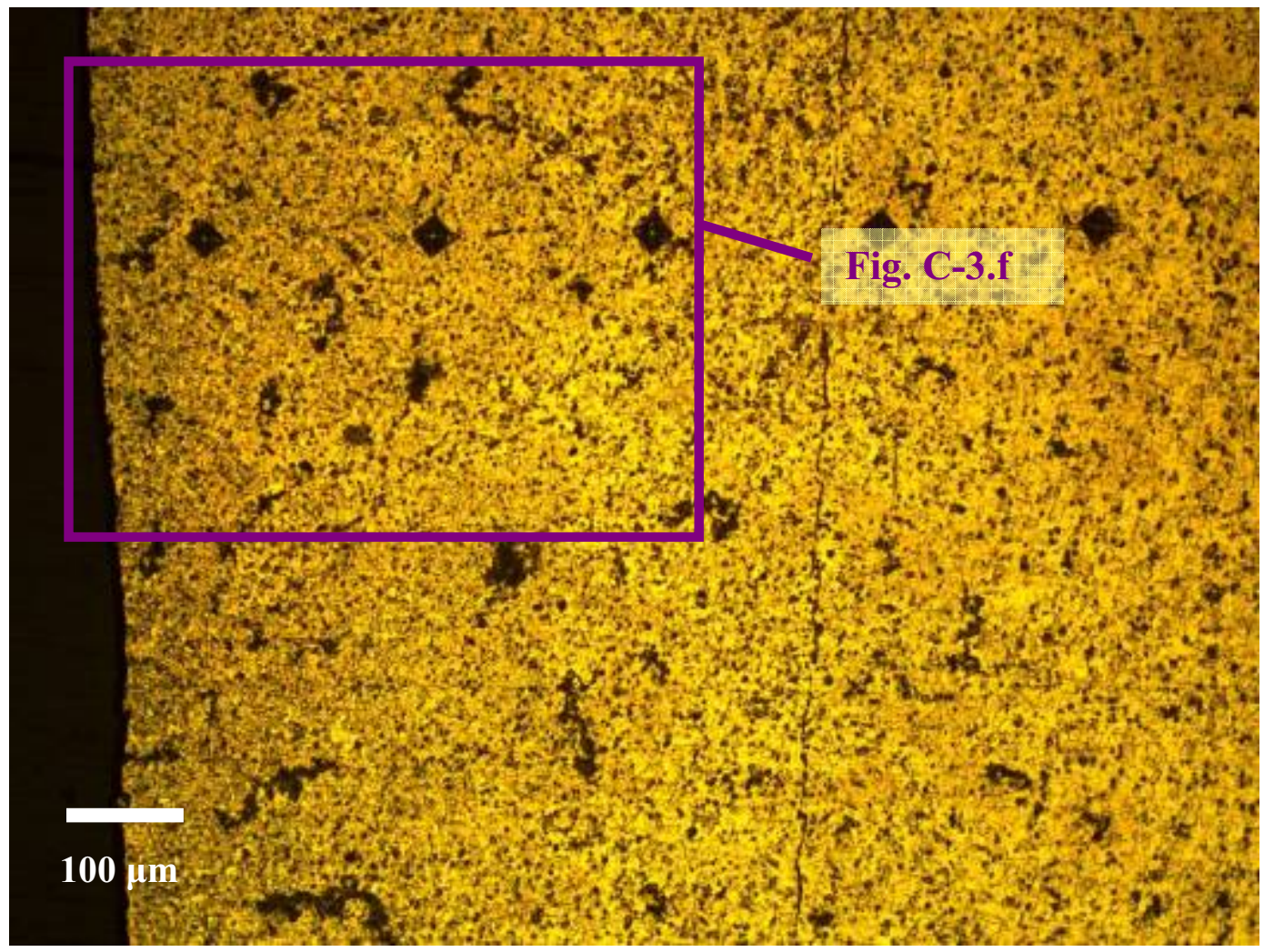

Figure C-3.e FB side of "retreating edge" location on etched aluminum sample with 1-sided, 1-pass FB fabricated with FB tool load of $53.4 \mathrm{kN}(12,000 \mathrm{lbf}), 100 \mathrm{X}$.

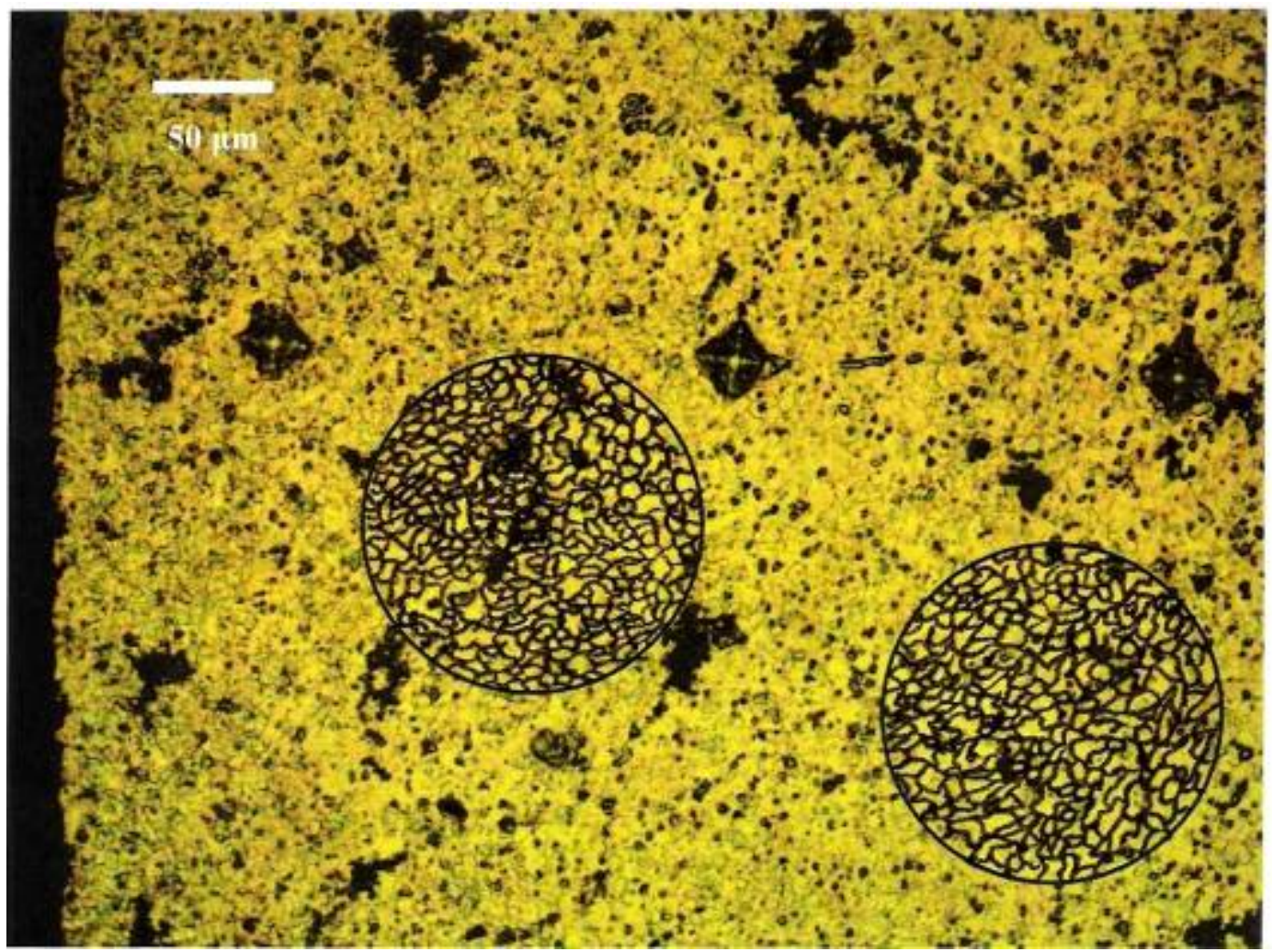

Figure C-3.f FB side of "retreating edge" location on etched aluminum sample, FB tool load of $53.4 \mathrm{kN}$ (12,000 lbf), 200X, grain size measurements. Avg. for 2 circles: $265^{1} / 2$ grains within circle $+(50$ grains cut by circumf. $) / 2=290 \frac{1}{2}$ grains. So $\mathrm{N}=290 \frac{1}{2}$ grains $/ 0.25 \mathrm{in}^{2}=1162$ grains $/ 1$ in $^{2}=2^{(\mathrm{G}-1)} \rightarrow \mathrm{G}=\ln 1162 / \ln 2+1=11.2$. 


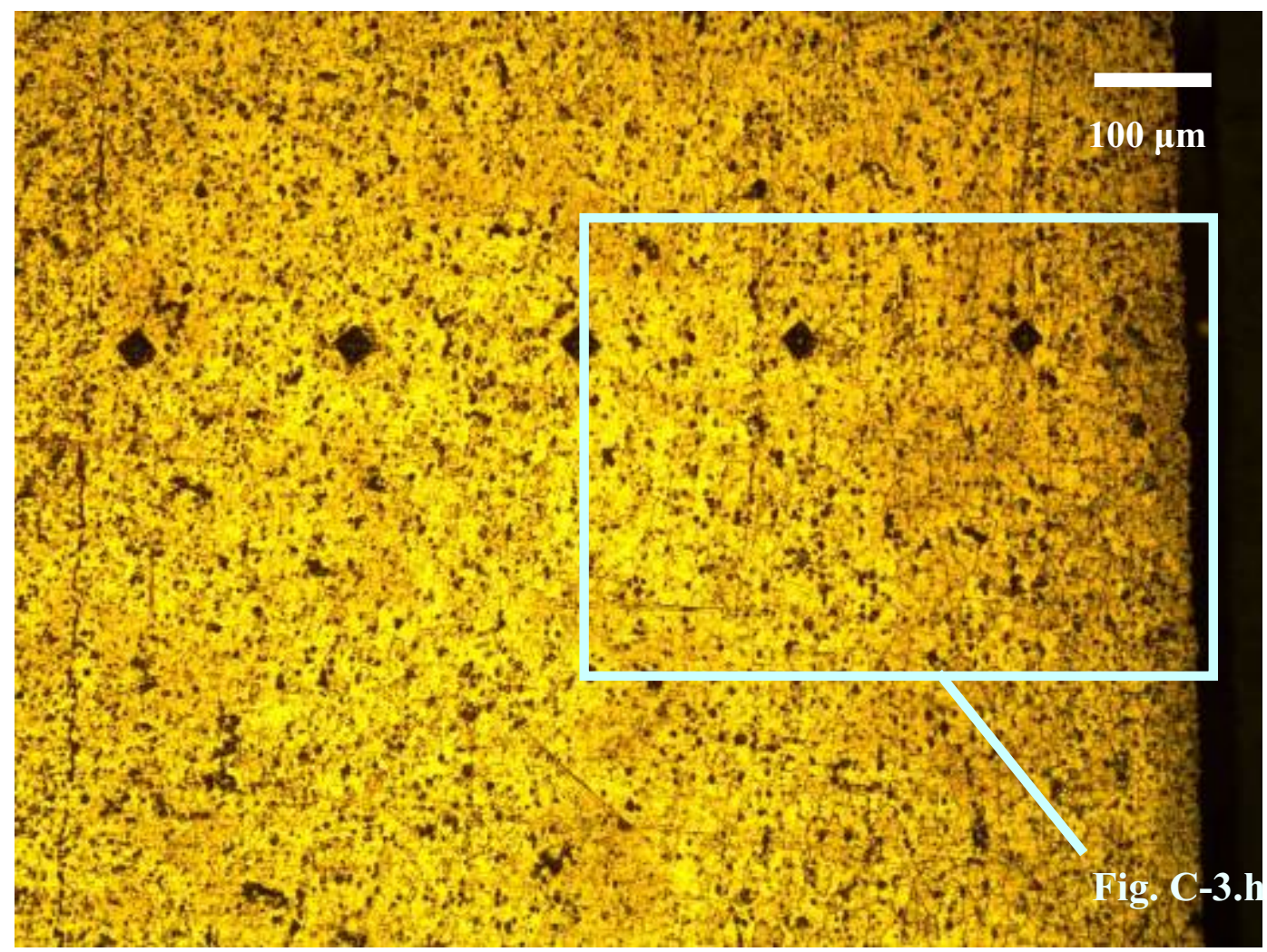

Figure C-3.g Non-FB (smooth) side of "retreating edge" location on etched aluminum sample with 1-sided, 1-pass FB fabricated with FB tool load of $53.4 \mathrm{kN}(12,000 \mathrm{lbf}), 100 \mathrm{X}$.

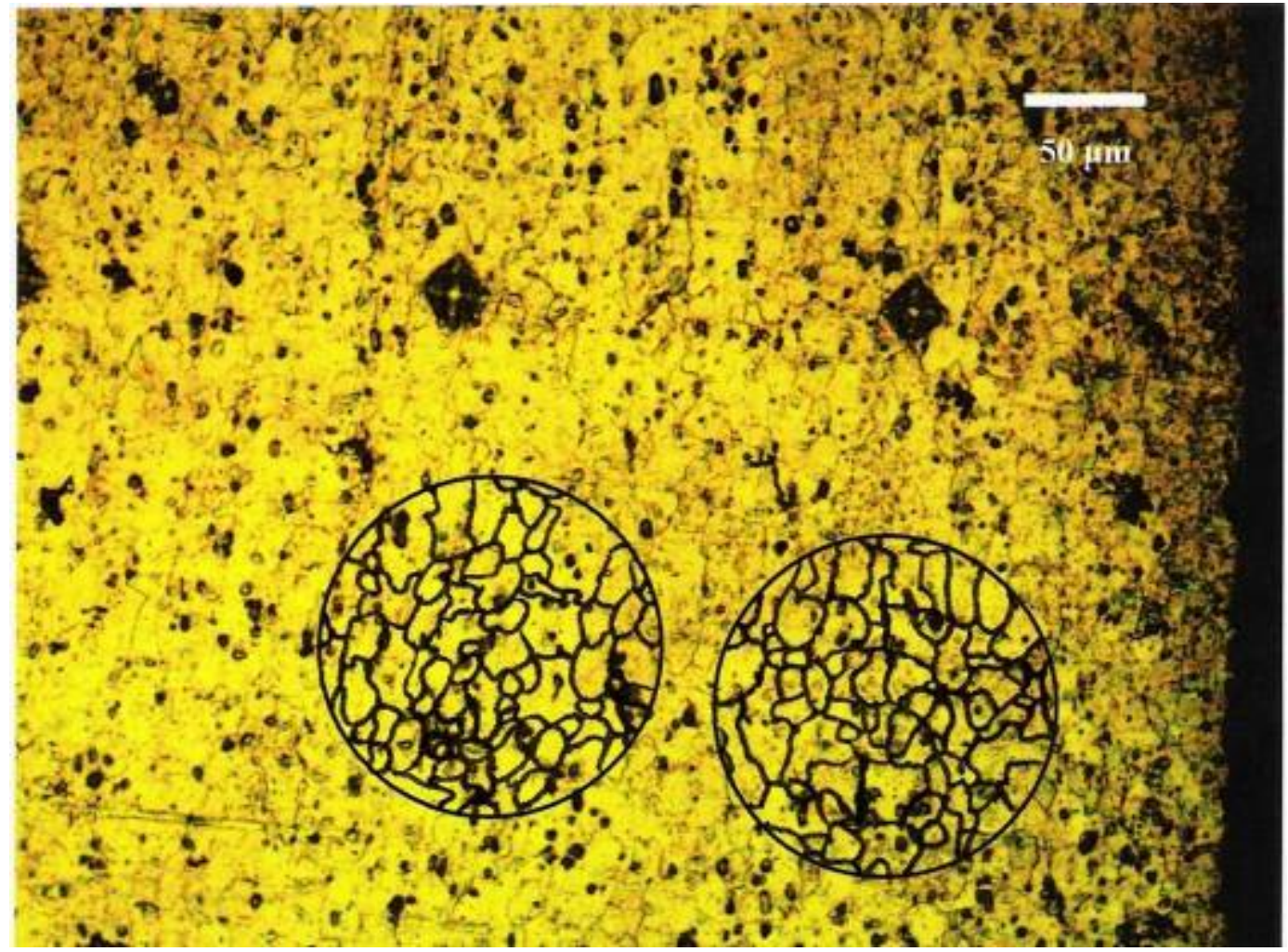

Figure C-3.h Non-FB side of "retreating edge" location on etched aluminum sample, FB tool load of $53.4 \mathrm{kN}$ (12,000 lbf), 200X, grain size measurements. Avg. for 2 circles: $56^{1} / 2$ grains within circle $+(30$ grains cut by circumf. $) / 2=71 \frac{1}{2}$ grains. So $\mathrm{N}=71 \frac{1}{2}$ grains $/ 0.25 \mathrm{in}^{2}=286$ grains $/ 1$ in $^{2}=2^{(\mathrm{G}-1)} \rightarrow \mathrm{G}=\ln 286 / \ln 2+1=9.2$. 


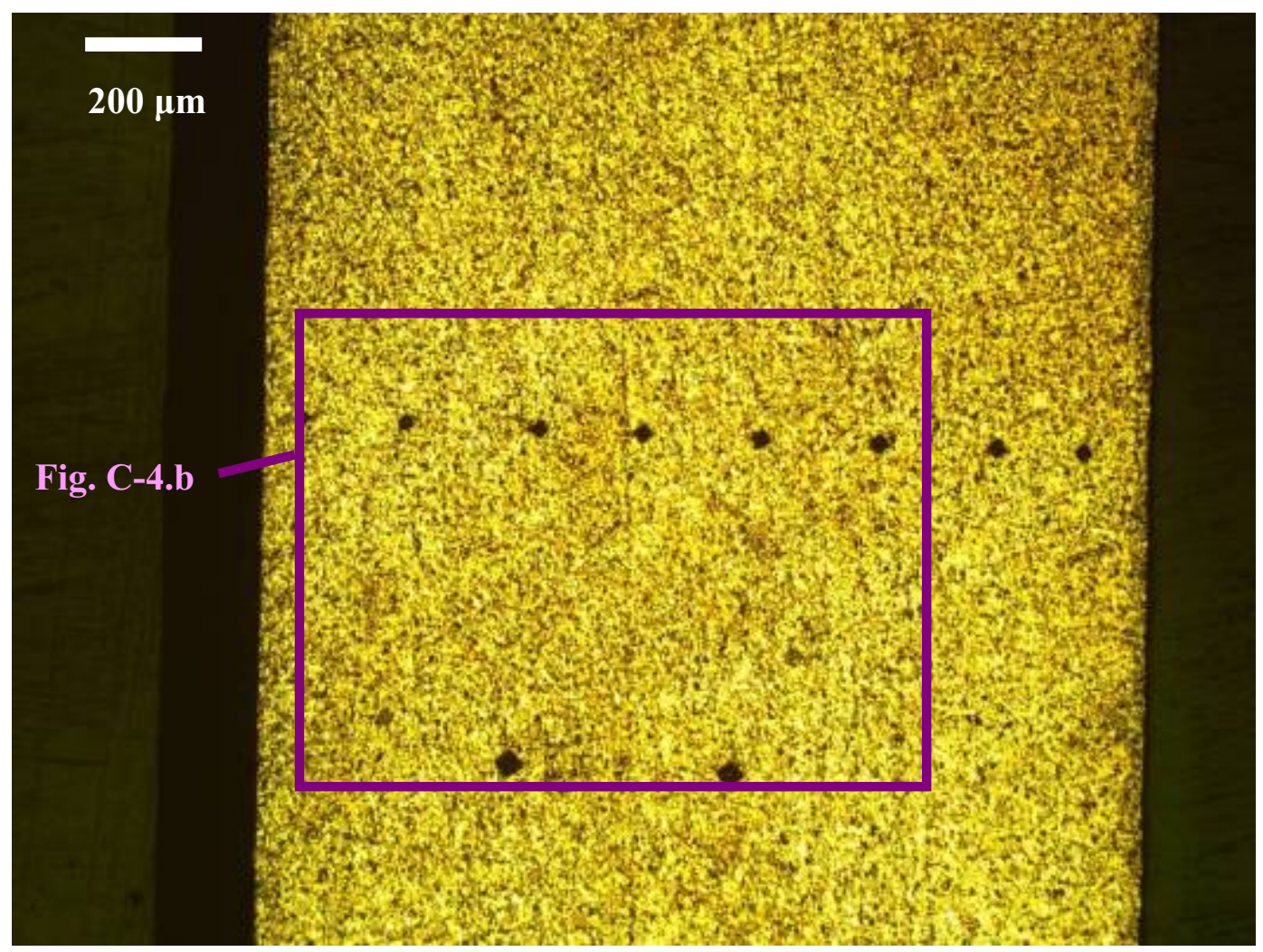

Figure C-4.a "Center" location on etched aluminum sample with 1-sided, 1-pass FB fabricated with FB load of $62.3 \mathrm{kN}(14,000 \mathrm{lbf}), 50 \mathrm{X}$.

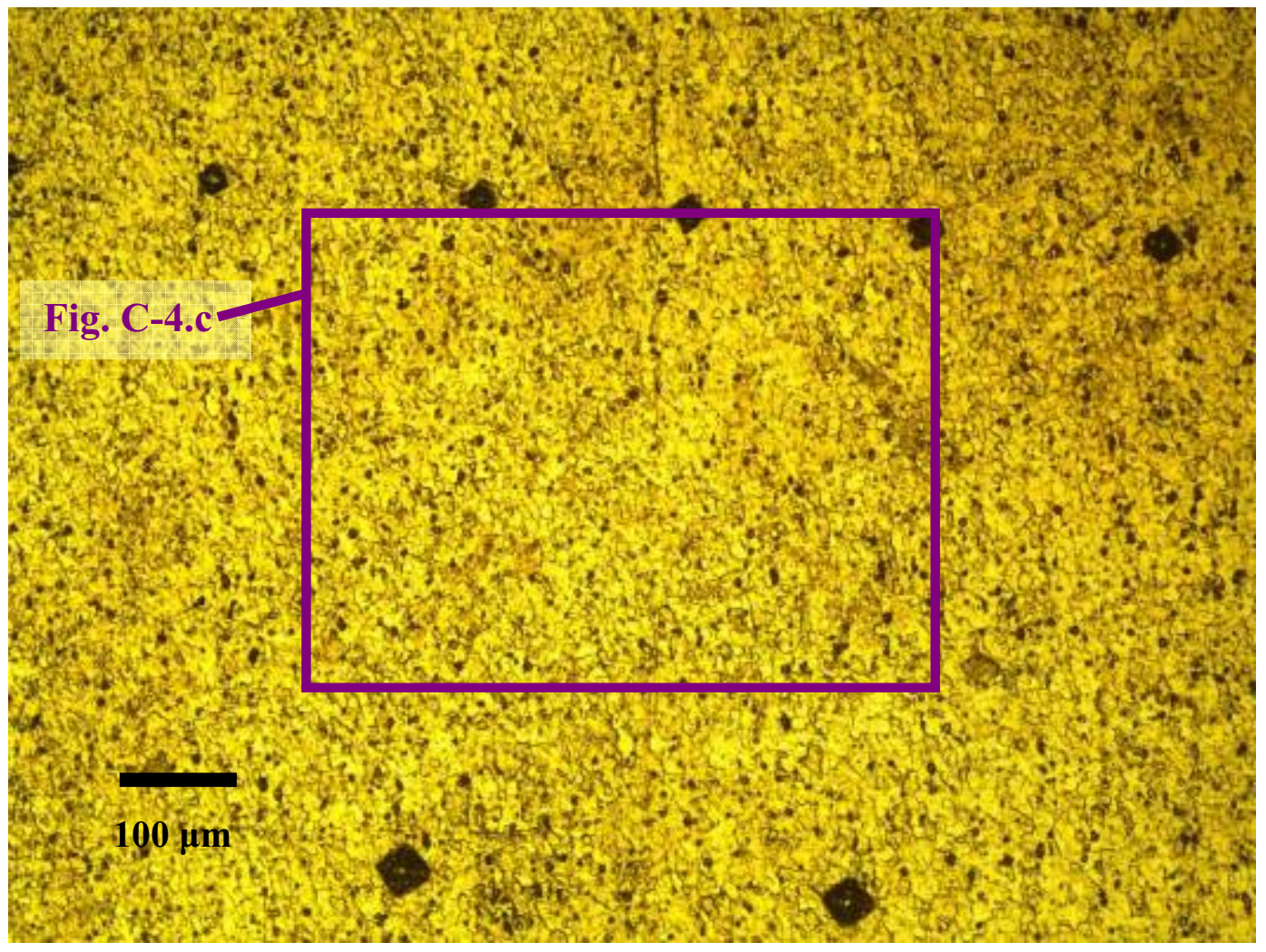

Figure C-4.b "Center" location on etched aluminum sample with 1-sided, 1-pass FB fabricated with FB tool load of $62.3 \mathrm{kN}(14,000 \mathrm{lbf}), 100 \mathrm{X}$. 


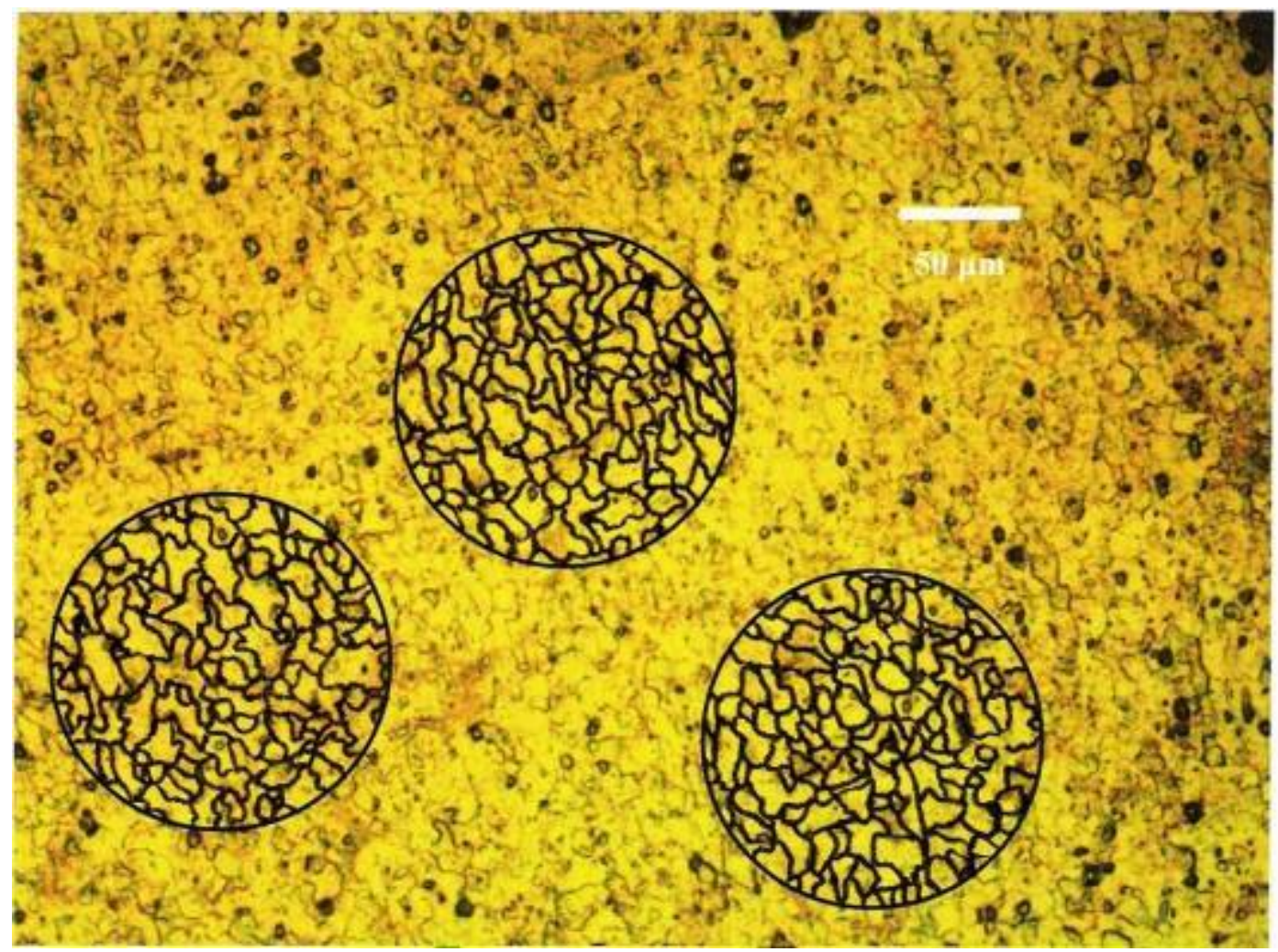

Figure C-4.c "Center" location on etched aluminum sample, FB tool load of $62.3 \mathrm{kN}(14,000$ lbf), 200X, grain size measurements. Avg. for 3 circles: $93^{2} / 3$ grains within circle $+\left(38^{1} / 3\right.$ grains cut by circumf. $) / 2=112^{5} / 6$ grains. So $\mathrm{N}=112^{5} / 6$ grains $/ 0.25 \mathrm{in}^{2}=451 \frac{1}{3}$ grains $/ 1 \mathrm{in}^{2}=2^{(\mathrm{G}-1)} \rightarrow$ $\mathrm{G}=\ln 451^{1} / 3 / \ln 2+1=9.8$.

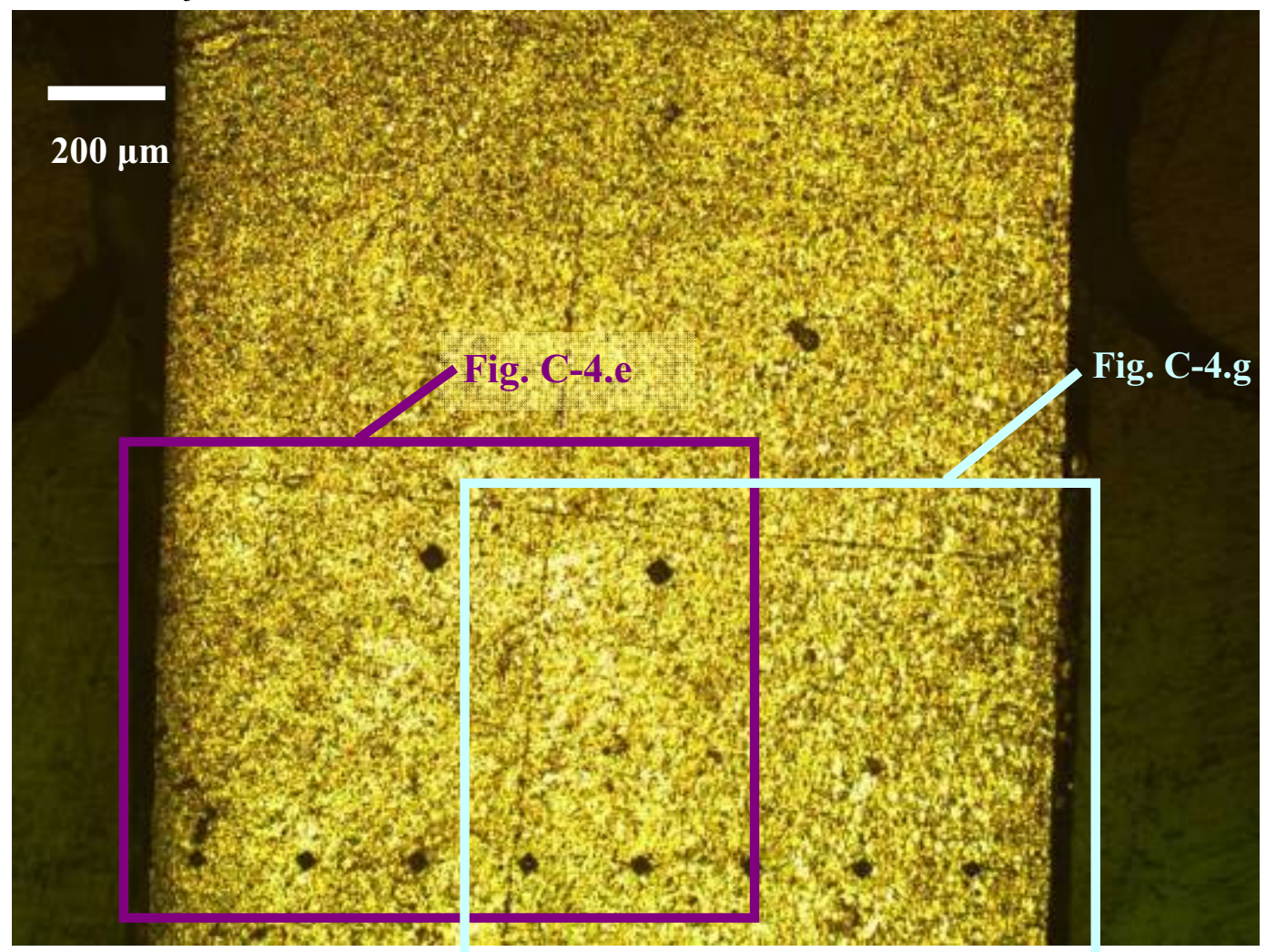

Figure C-4.d "Retreating edge" location on etched aluminum sample with 1-sided, 1-pass FB fabricated with tool load of $62.3 \mathrm{kN}$ (14,000 lbf), 50X. 


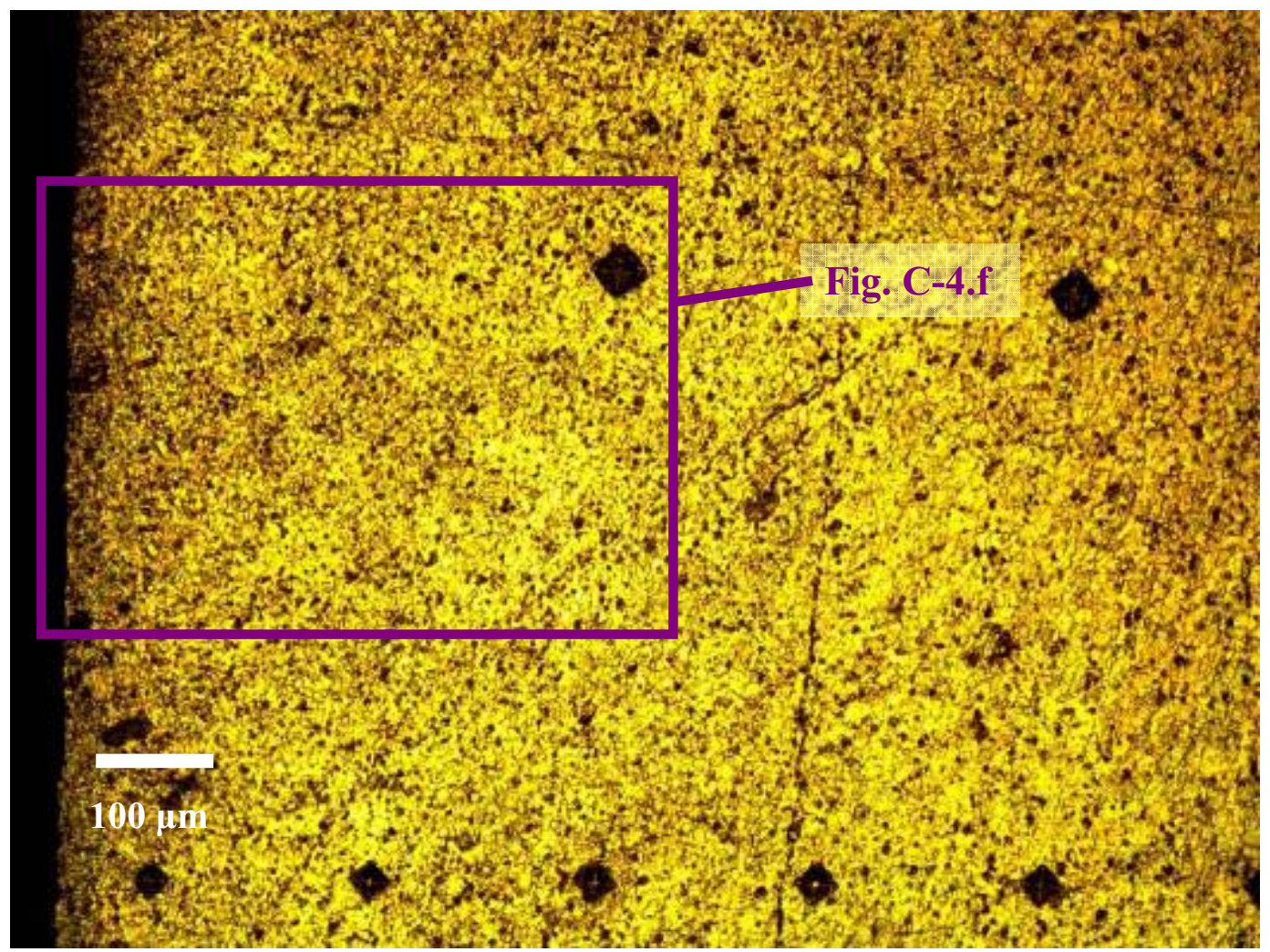

Figure C-4.e FB side of "retreating edge" location on etched aluminum sample with 1-sided, 1-pass FB fabricated with tool load of $62.3 \mathrm{kN}(14,000 \mathrm{lbf})$ pressure, 100X.

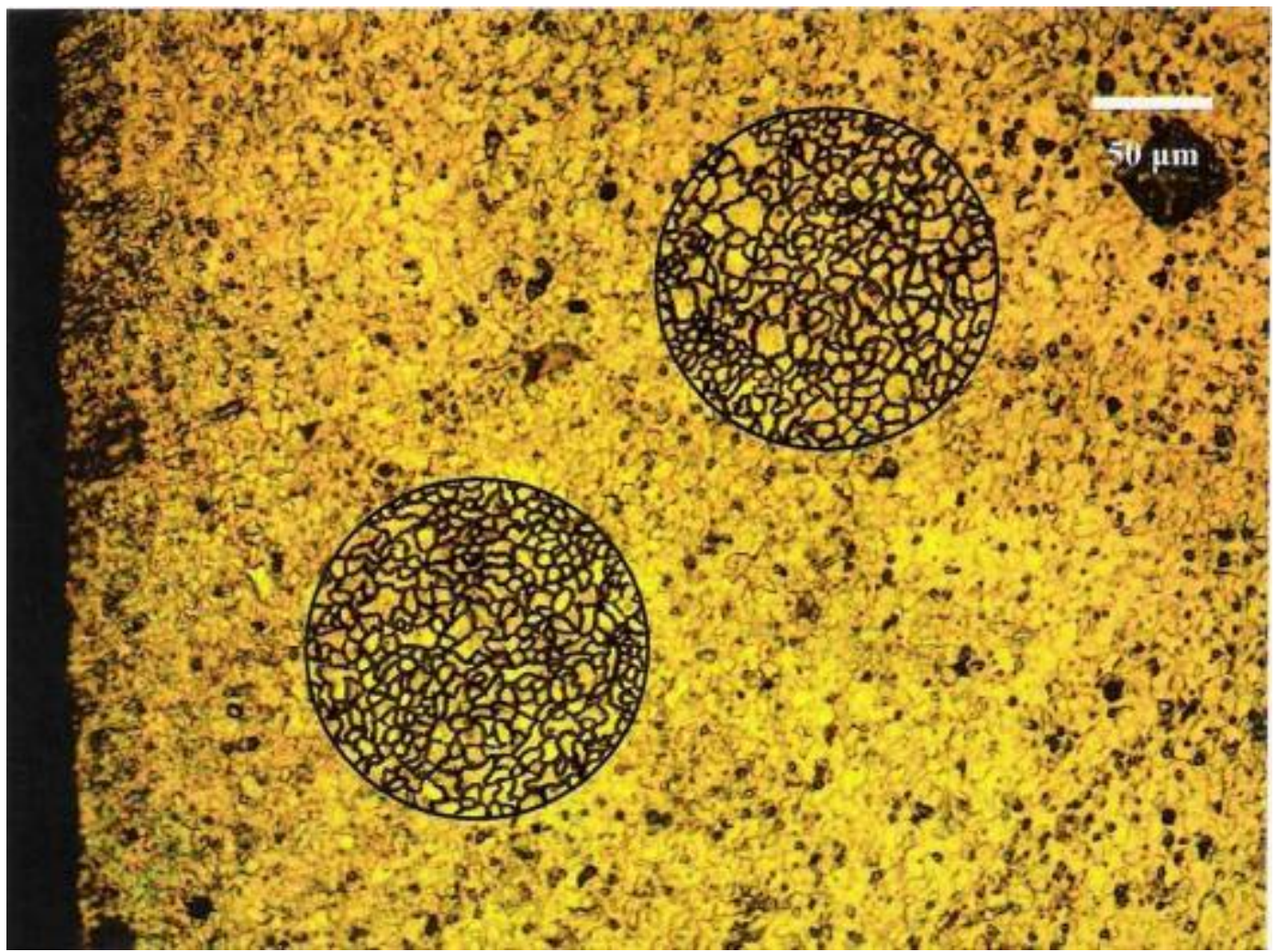

Figure C-4.f FB side of "retreating edge" location on etched aluminum sample, FB tool load of $62.3 \mathrm{kN}(14,000 \mathrm{lbf}), 200 \mathrm{X}$, grain size measurements. Avg. for 2 circles: $265^{1} / 2$ grains within circle $+\left(60 \frac{1}{2}\right.$ grains cut by circumf. $) / 2=295^{3} / 4$ grains. So $\mathrm{N}=295^{3} / 4$ grains $/ 0.25 \mathrm{in}^{2}=1183$ grains $/ 1$ in $^{2}=2^{(\mathrm{G}-1)} \rightarrow \mathrm{G}=\ln 1183 / \ln 2+1=11.2$. 


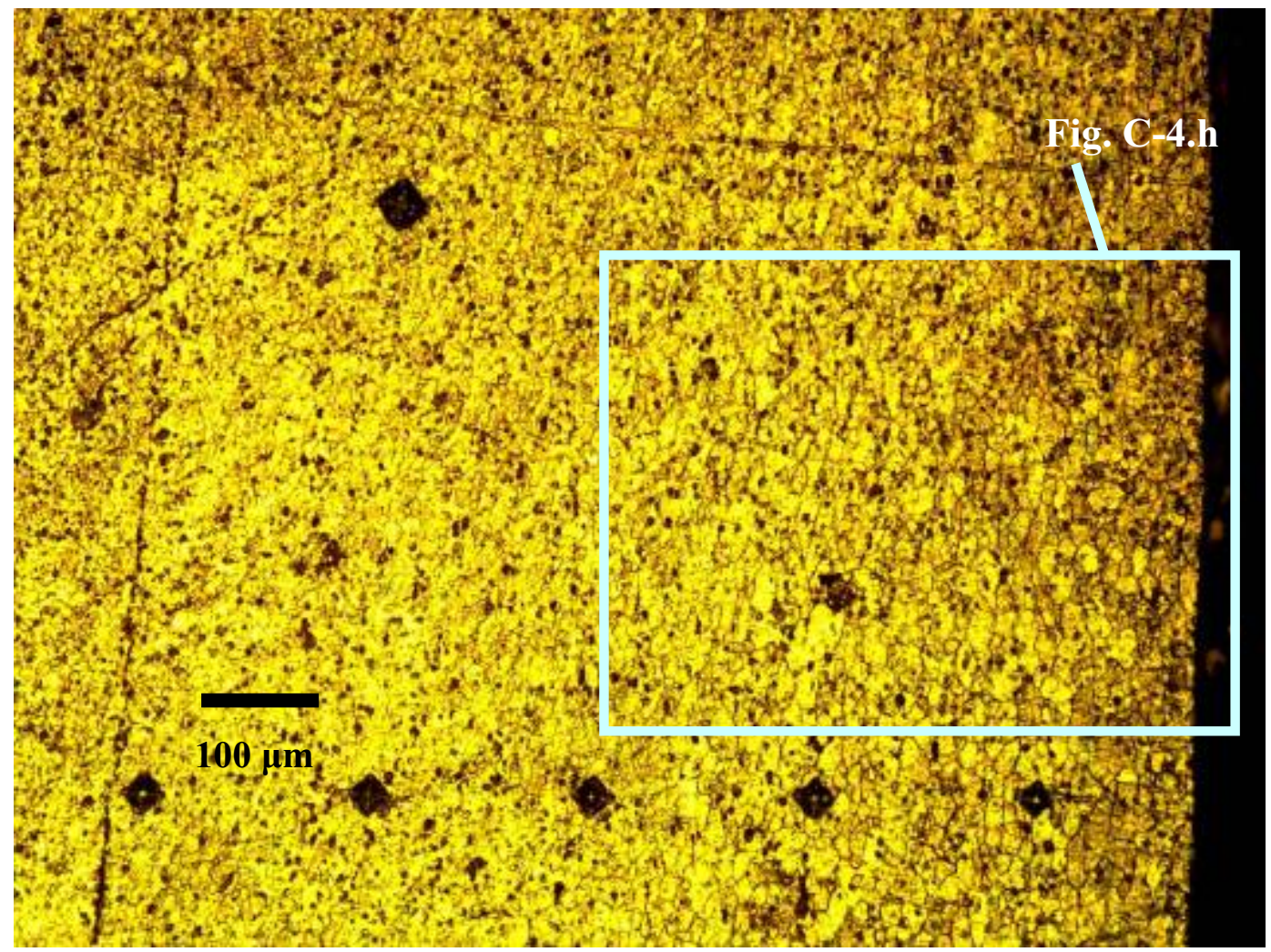

Figure C-4.g Non- FB side of "retreating edge" location on etched aluminum sample with 1-sided, 1-pass FB fabricated with tool load of $62.3 \mathrm{kN}$ (14,000 lbf) pressure, 100X.

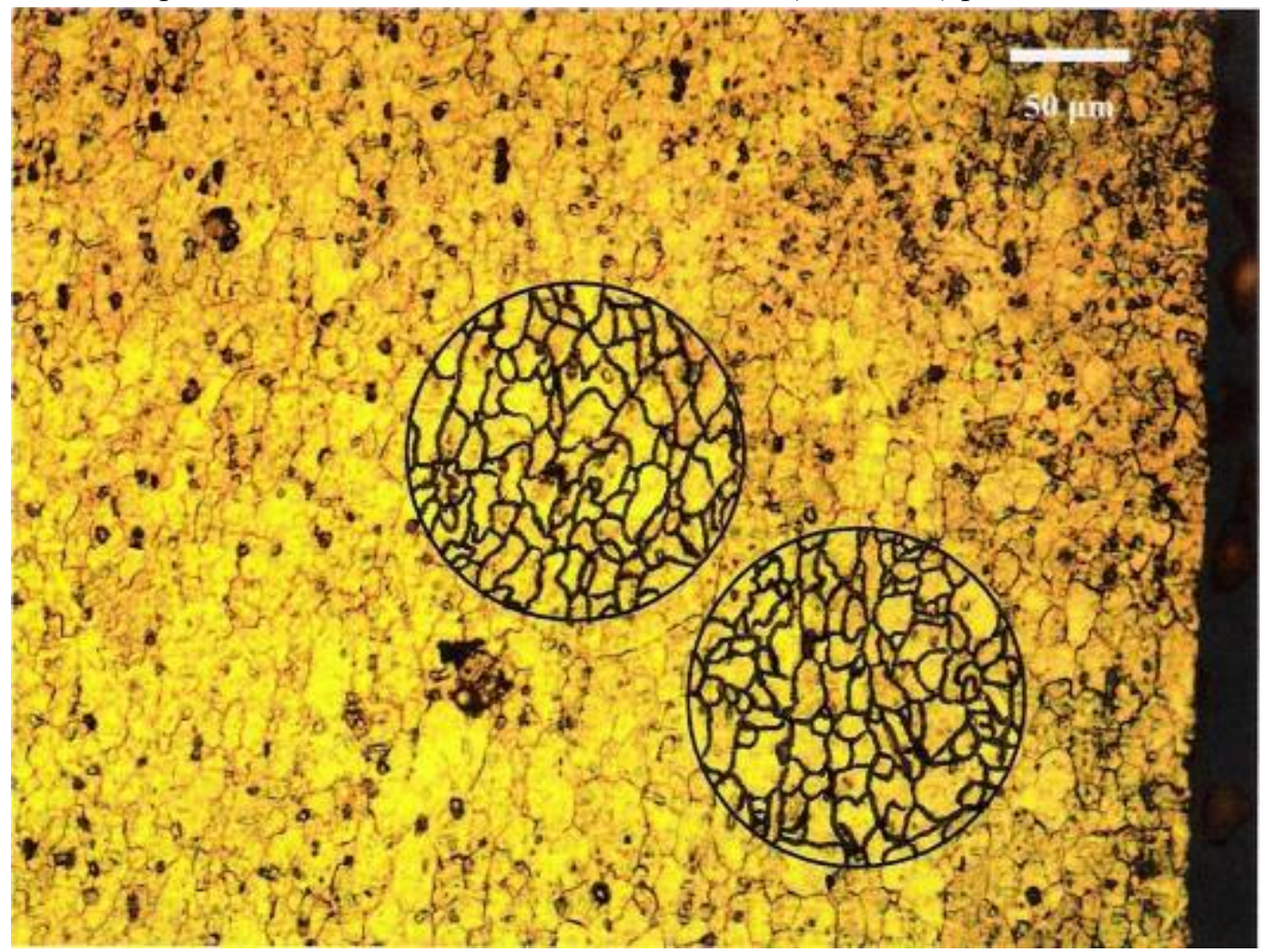

Figure C-4.h Non-FB side of "retreating edge" location on etched aluminum sample, FB tool load of $62.3 \mathrm{kN}(14,000 \mathrm{lbf}), \mathbf{2 0 0 X}$, grain size measurements. Avg. for 2 circles: 83 grains within circle $+(37$ grains cut by circumf. $) / 2=101^{1} / 2$ grains. $\mathrm{So} \mathrm{N}=101 \frac{1}{2}$ grains $/ 0.25 \mathrm{in}^{2}=406$ grains $/ 1$ in $^{2}=2^{(\mathrm{G}-1)} \rightarrow \mathrm{G}=\ln 406 / \ln 2+1=9.7$. 


\title{
Microstructural Examination to Aid in Understanding \\ Friction Bonding Fabrication Technique \\ for Monolithic Nuclear Fuel
}

\begin{abstract}
APPENDIX D
Microphotos of DU10Mo Monolithic Fuel Plate Samples

(See Figure 5.6 in main report text for microphoto locations on sample)
\end{abstract}

$\underline{\text { Figure }}$

D-1 Microphotos of Sample Fabricated with FB Tool Face of Steel-A36

D-2 Microphotos of Sample Fabricated with FB Tool Face of Aluminum-Bronze Alloy

D-3 Microphotos of Sample Fabricated with FB Tool Face of Anviloy 
APPENDIX D

Microphotos of DU10Mo Monolithic Fuel Plate Samples

(page is intentionally blank) 


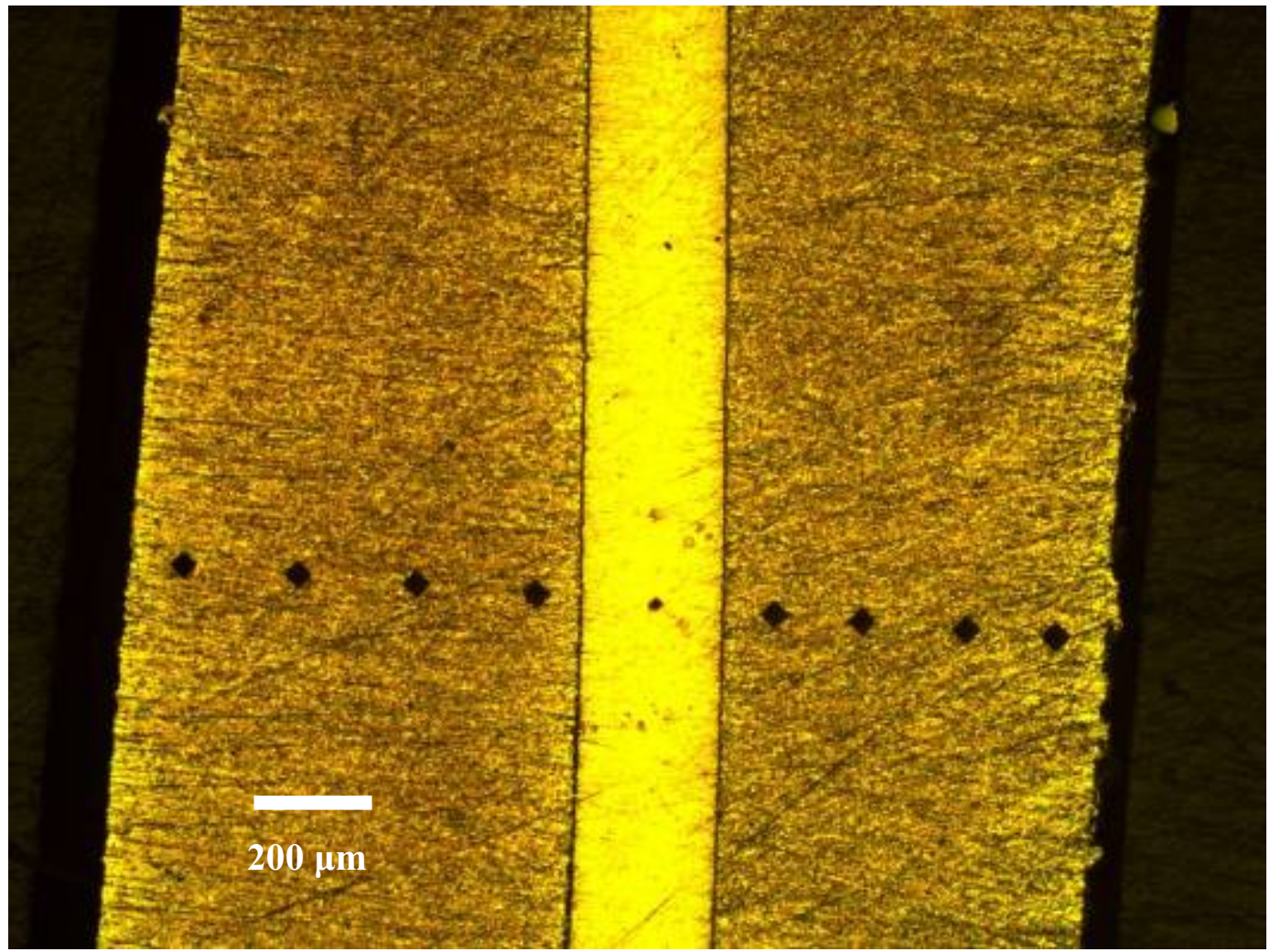

Figure D-1.a Bonded location near top of sample with DU10Mo foil in Al-6061 cladding, bonded by multiple-pass FB with Steel-A36 tool face, after etching was removed and hardness measurements taken (50X photo).

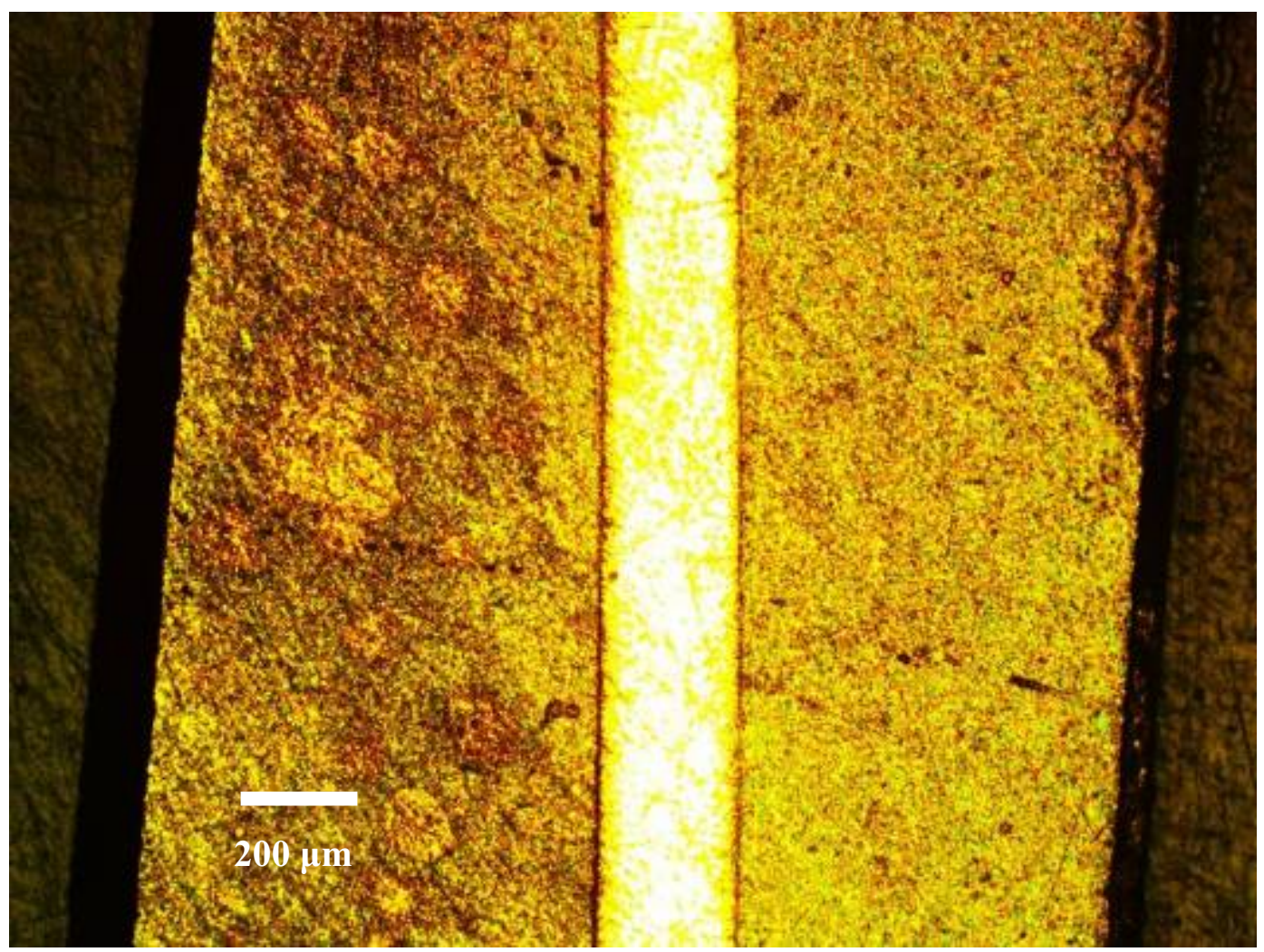

Figure D-1.b Same bonded location near top of etched sample with DU10Mo foil in Al-6061 cladding, bonded by multiple-pass FB with Steel-A36 tool face. Prior to re-polishing and hardness measurements (50X photo). 


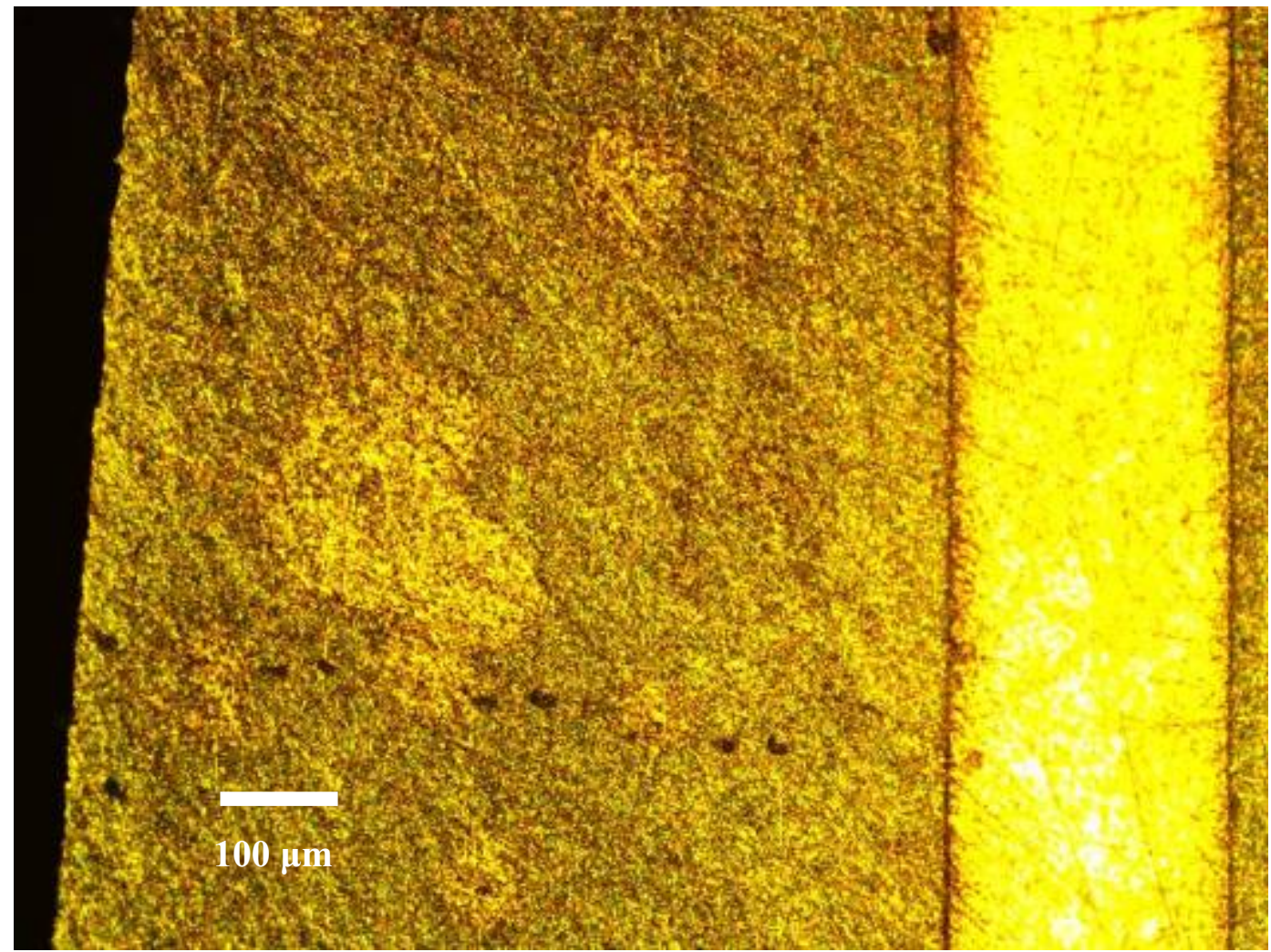

Figure D-1.c Bonded location near top of etched sample with DU10Mo foil in Al-6061 cladding, bonded by multiple-pass FB with Steel-A36 tool face (100X photo).

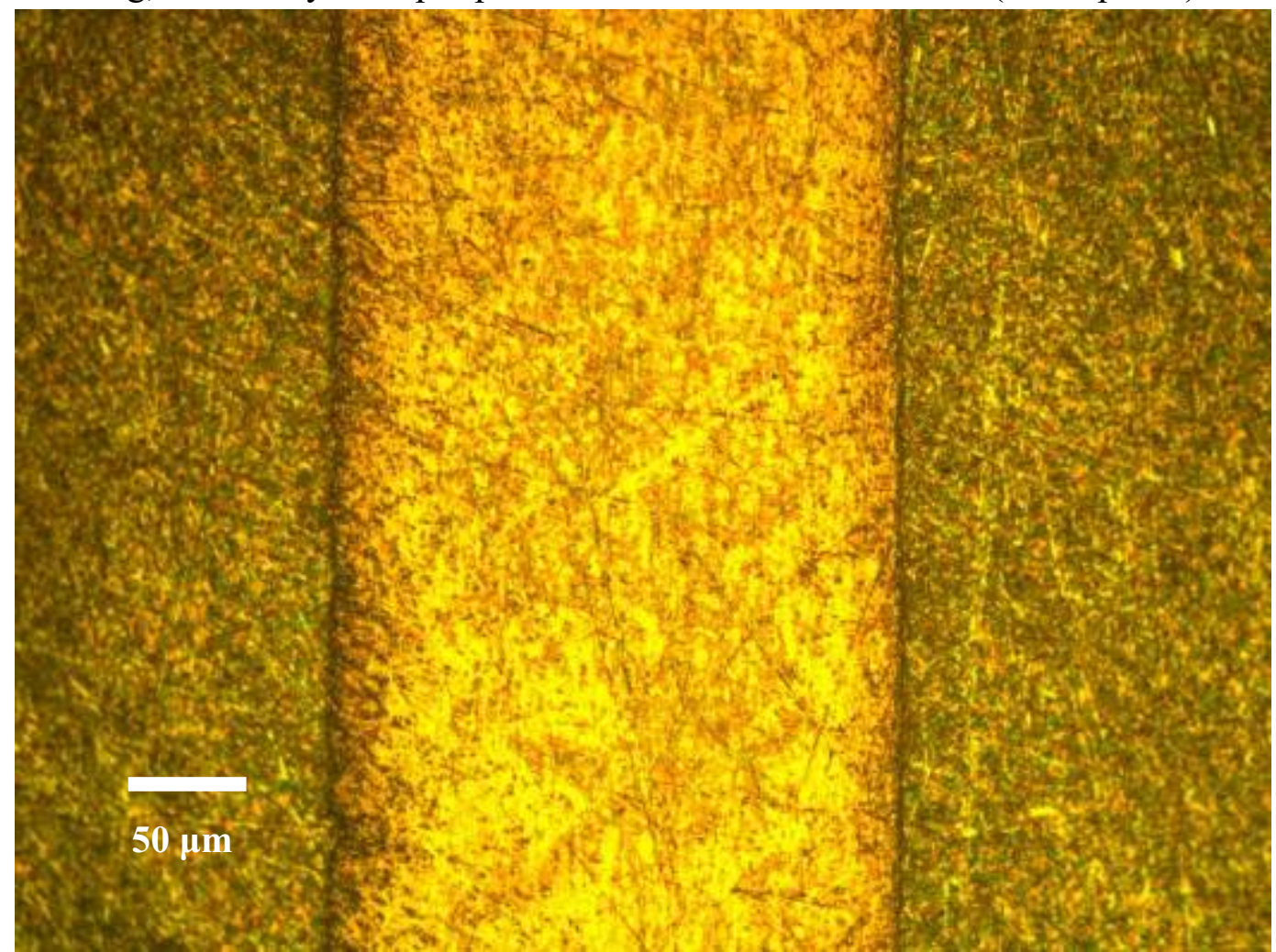

Figure D-1.d Bonded location near top of etched sample with DU10Mo foil in Al-6061 cladding, bonded by multiple-pass FB with Steel-A36 tool face (200X photo). 


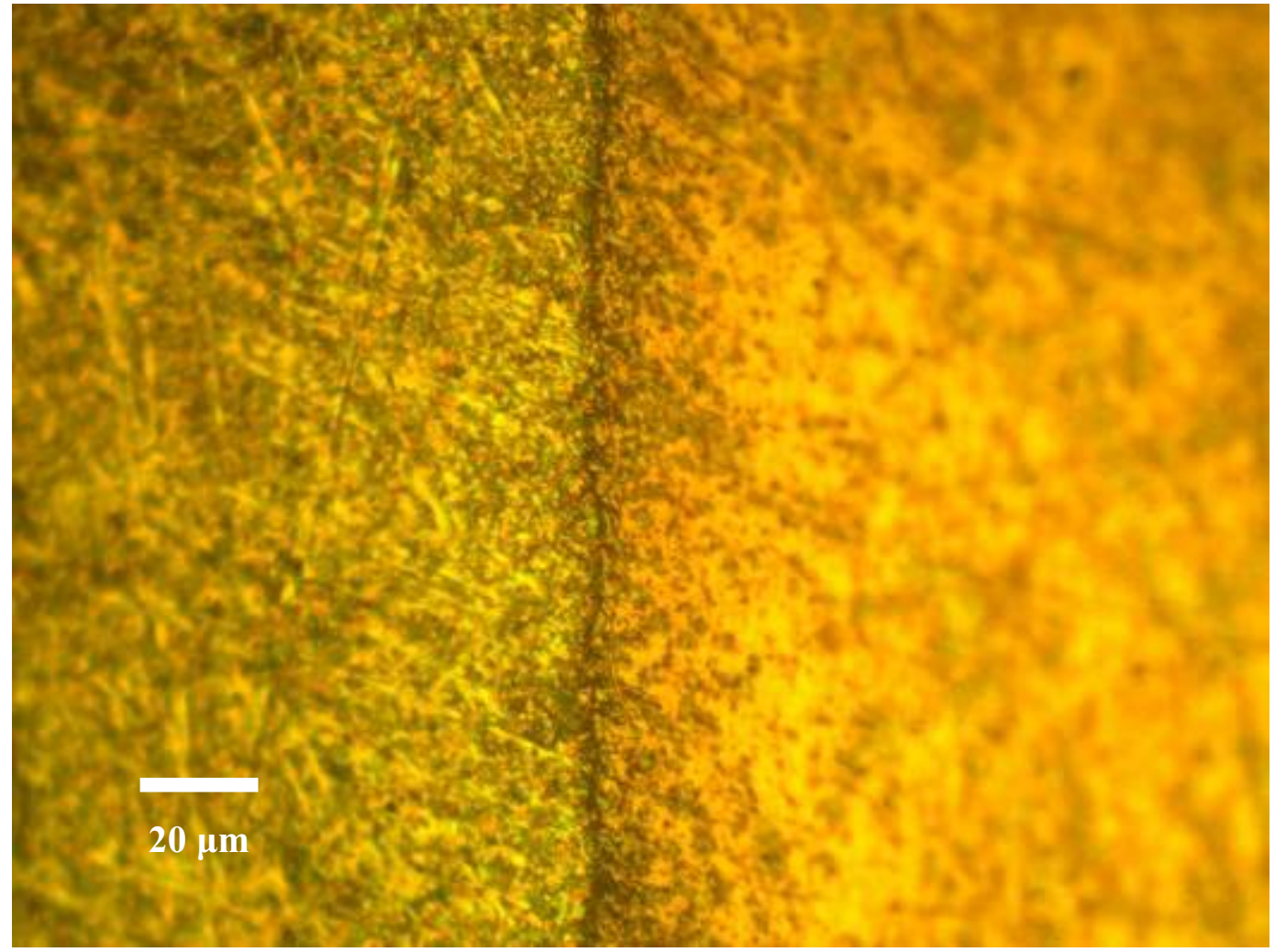

Figure D-1.e Bonded location near top of etched sample with DU10Mo foil in Al-6061 cladding, bonded by multiple-pass FB with Steel-A36 tool face (500X photo).

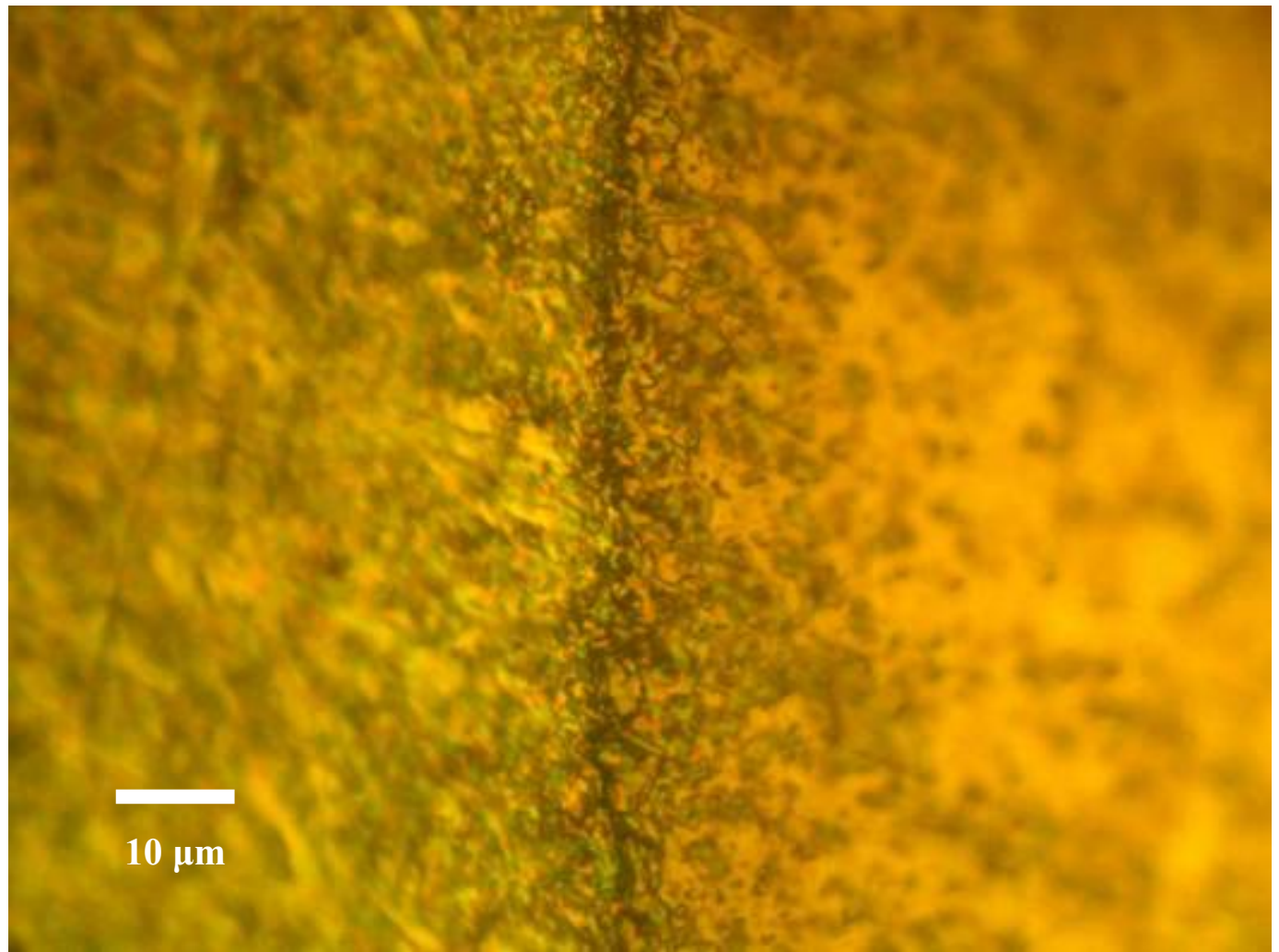

Figure D-1.f Bonded location near top of etched sample with DU10Mo foil in Al-6061 cladding, bonded by multiple-pass FB with tool face Steel-A36 (1000X photo). 


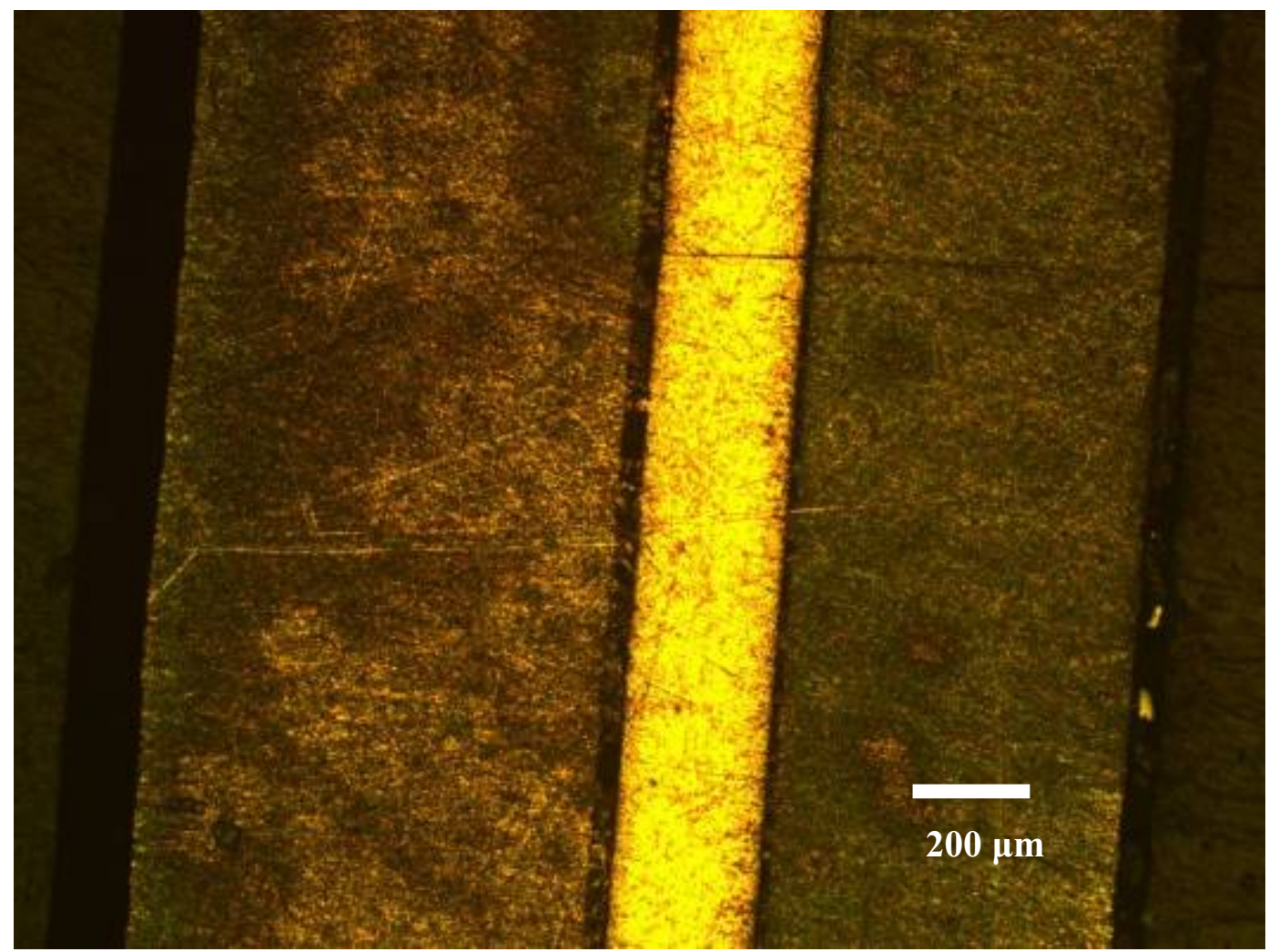

Figure D-1.g Typical debonded location on etched sample with DU10Mo foil in A1-6061 cladding, bonded by multiple-pass FB with Steel-A36 tool face (50X photo).

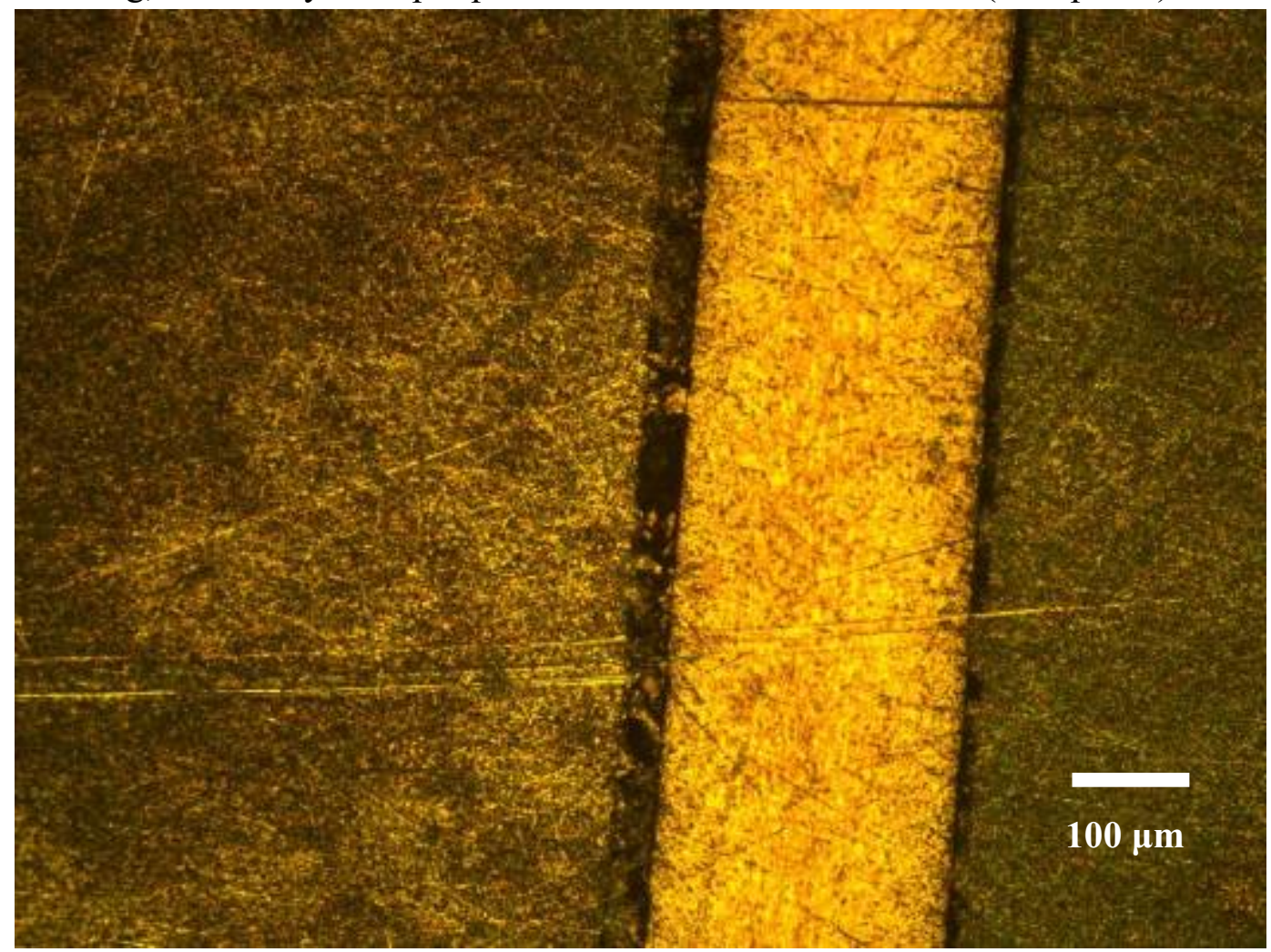

Figure D-1.h Typical debonded location on etched sample with DU10Mo foil in Al-6061 cladding, bonded by multiple-pass FB with Steel-A36 tool face (100X photo). 


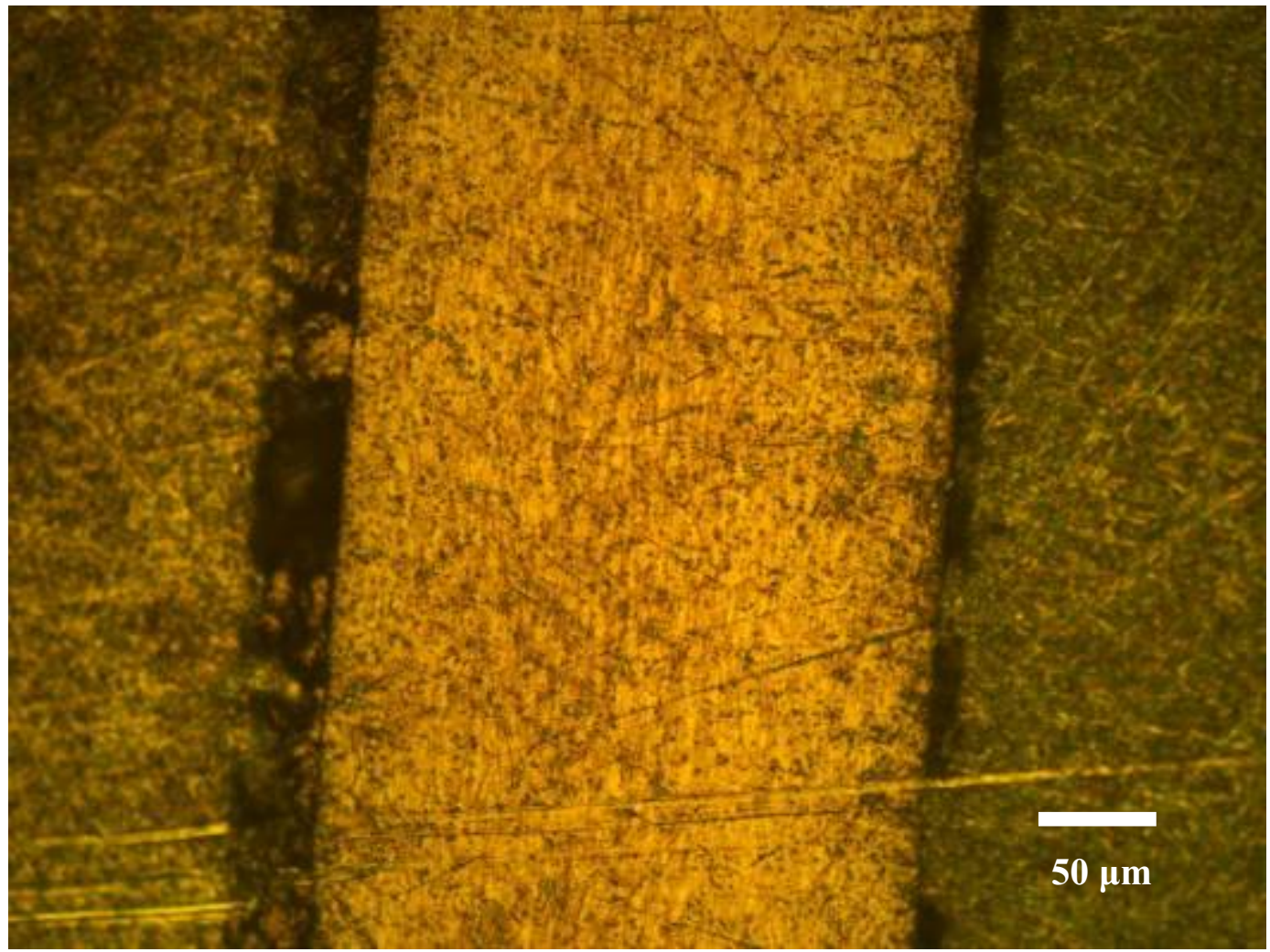

Figure D-1.i Typical debonded location on etched sample with DU10Mo foil in Al-6061 cladding, bonded by multiple-pass FB with Steel-A36 tool face (200X photo).
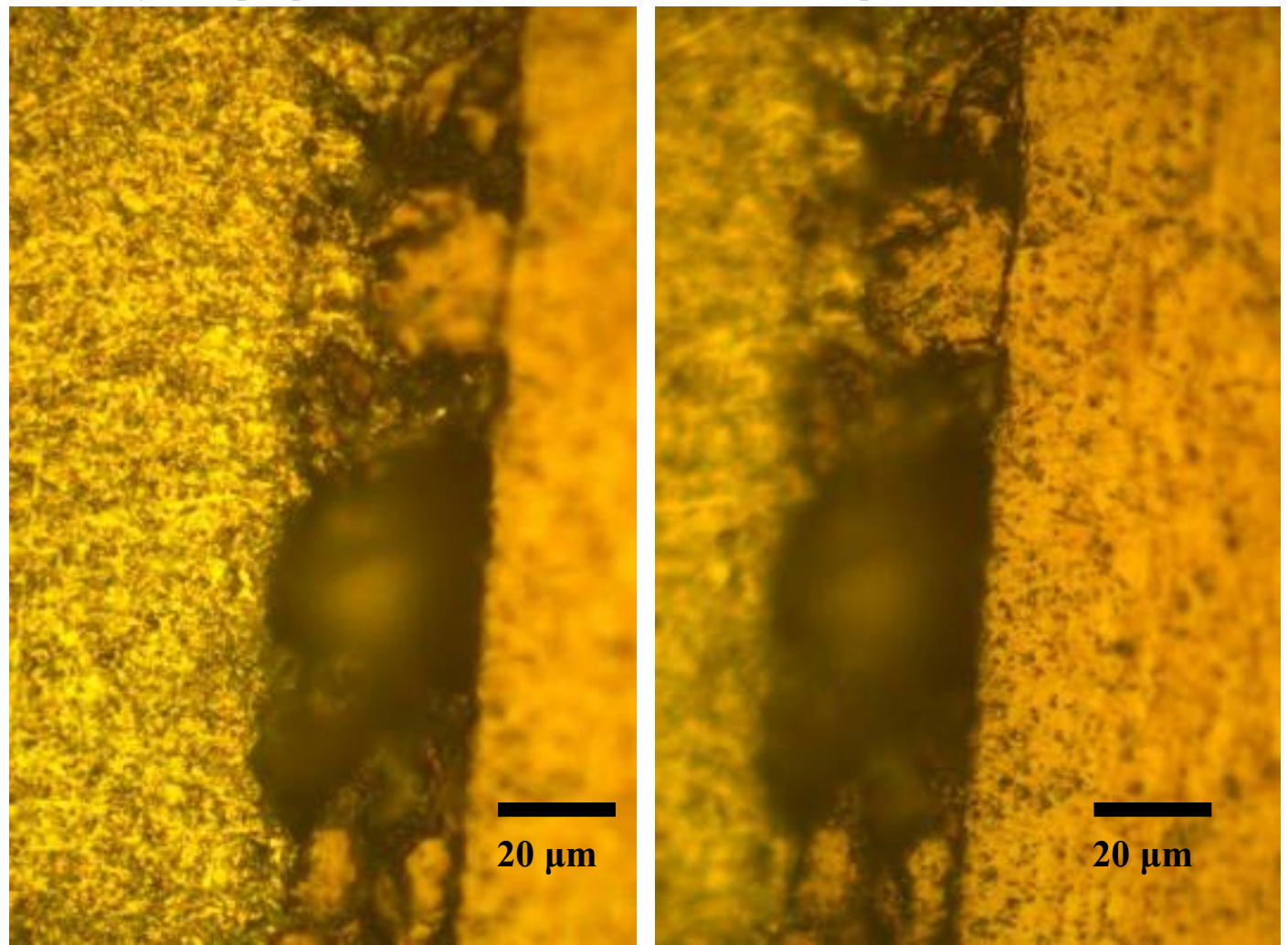

Figure D-1.j Typical debonded location on etched sample with DU10Mo foil in Al-6061 cladding, bonded by multiple-pass FB with Steel-A36 tool face (500X photos): aluminum in focus (left), and U10Mo in focus (right). 
APPENDIX D

Microphotos of DU10Mo Monolithic Fuel Plate Samples

(page is intentionally blank) 


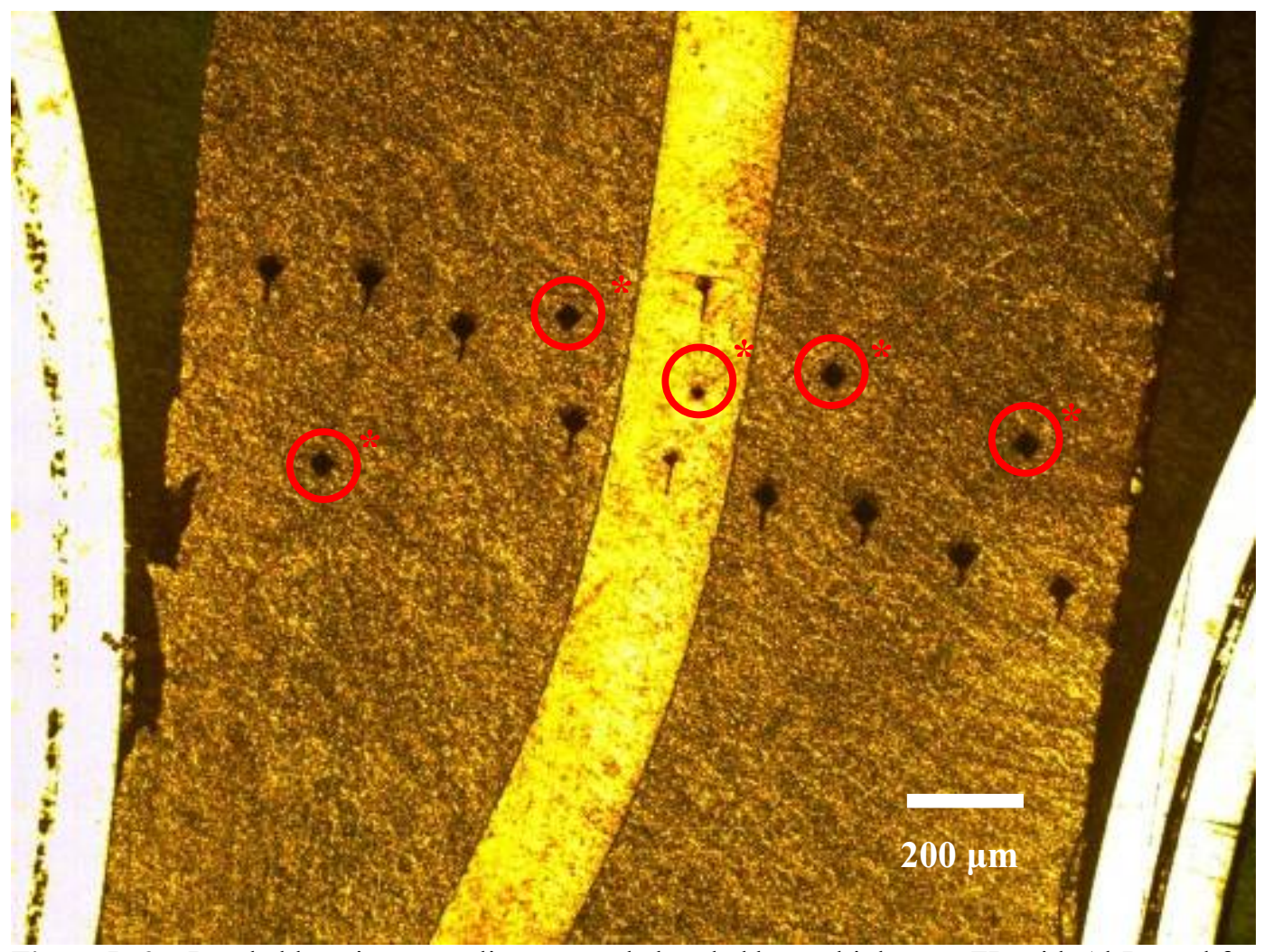

Figure D-2.a Bonded location near clip on sample bonded by multiple-pass FB with Al-Br tool face, after etching was removed and hardness measurements taken. First set of hardness indents at this location had "tails" due to unevenness on opposite side (50X photo).

* Five additional measurements, taken later after the sample's opposite side was polished flat, had no "tails," and had values consistent with prior measurements.

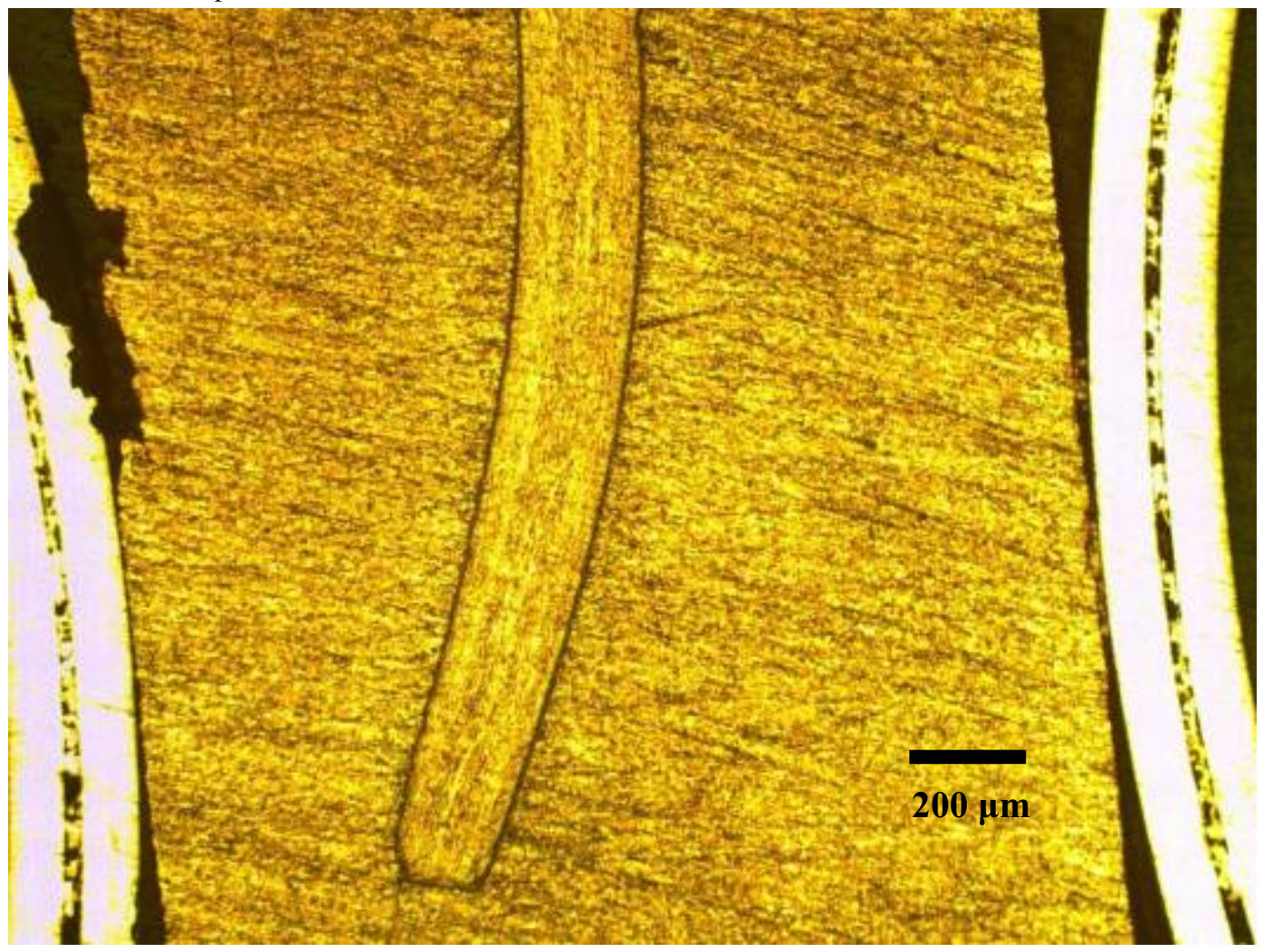

Figure D-2.b Same bonded location near clip end of etched sample with DU10Mo foil in Al-6061 cladding, bonded by multiple-pass FB with Al-Br tool face. Prior to re-polishing and hardness measurements. (50X photo) 


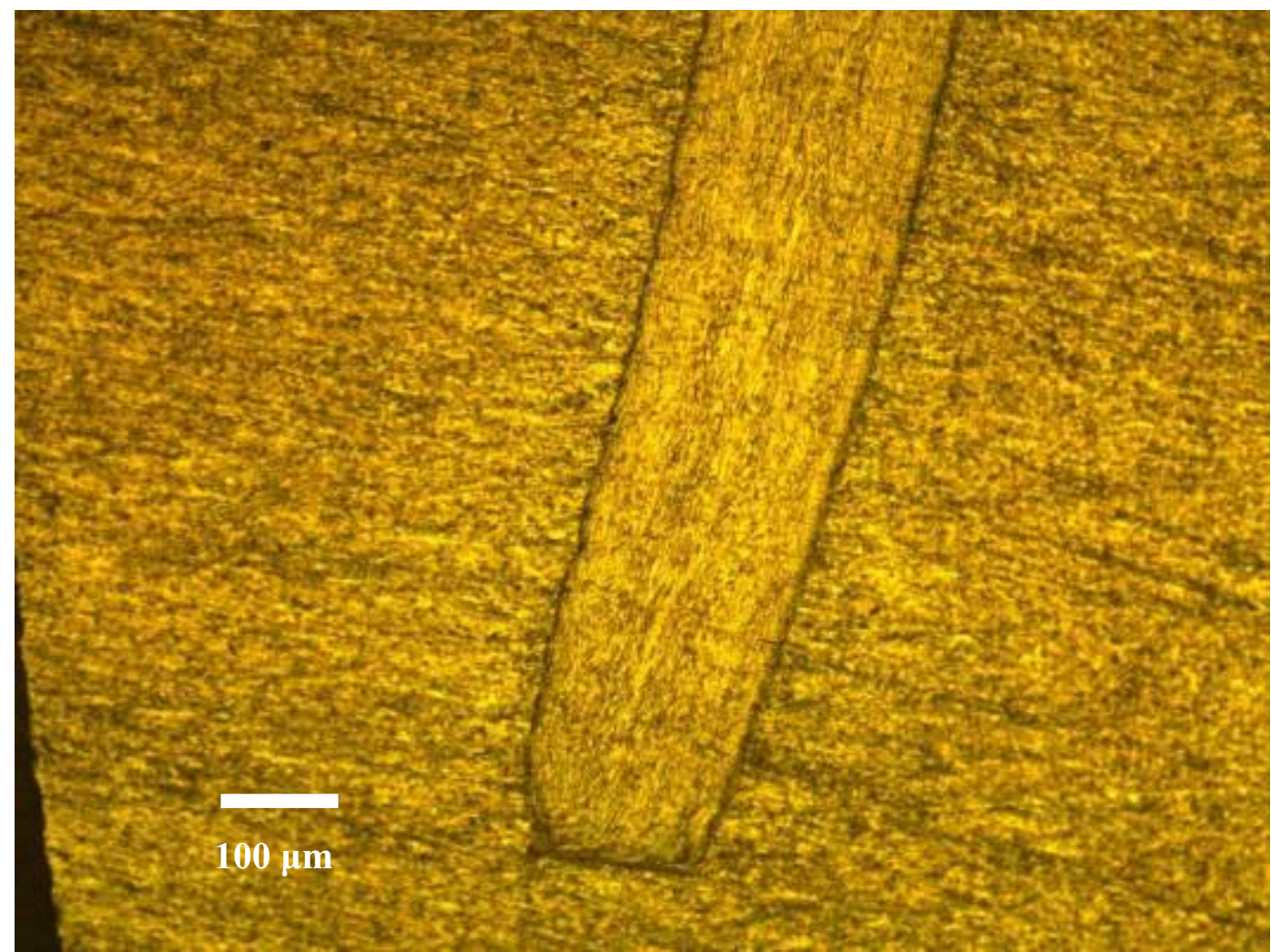

Figure D-2.c Bonded location near clip end etched sample with DU10Mo foil in Al-6061 cladding, bonded by multiple-pass FB with aluminum-bronze tool face (100X photo).

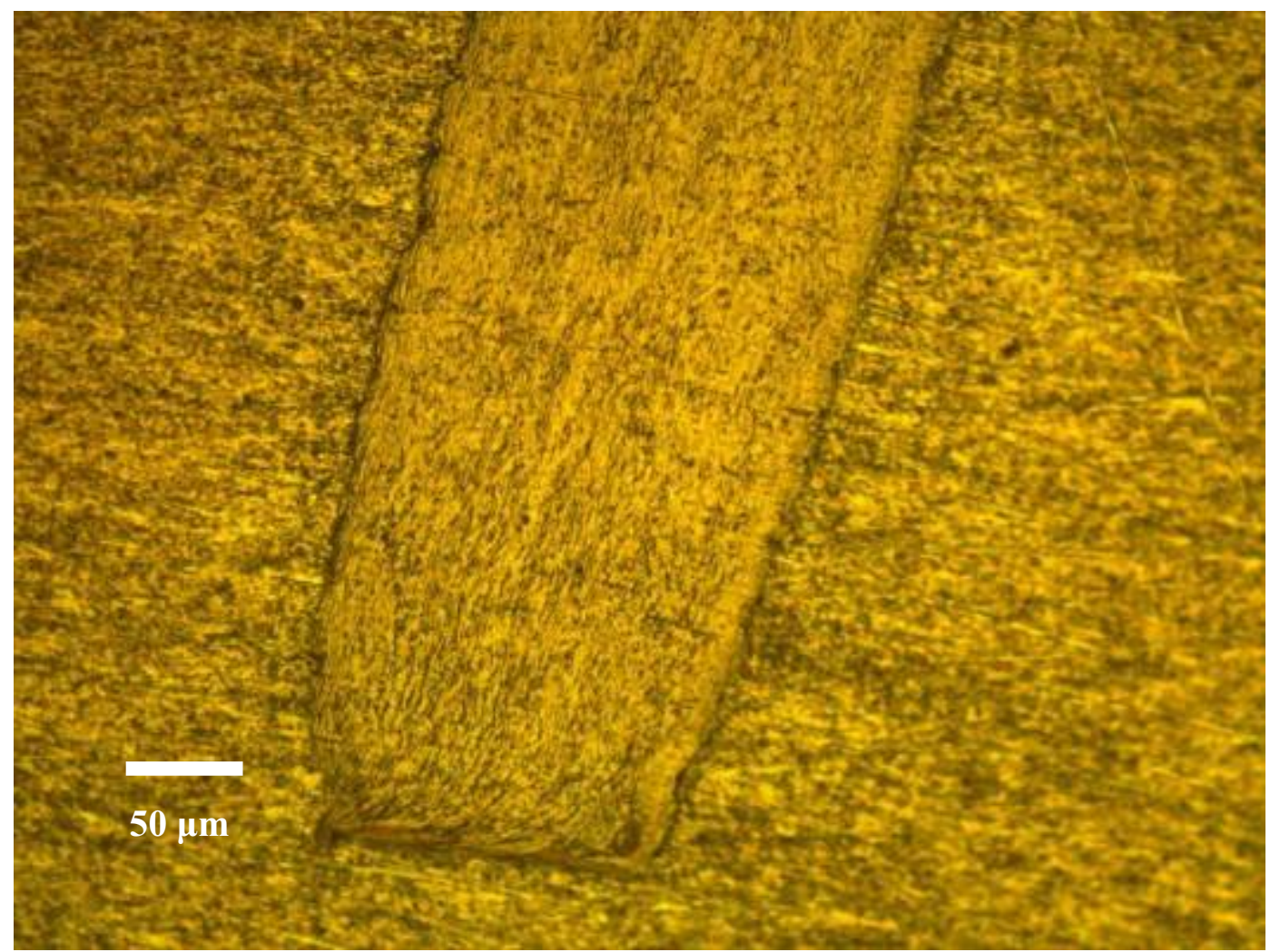

Figure D-2.d Bonded location near clip end of etched sample with DU10Mo foil in A1-6061 cladding, bonded by multiple-pass FB with tool face aluminum-bronze (200X photo). 


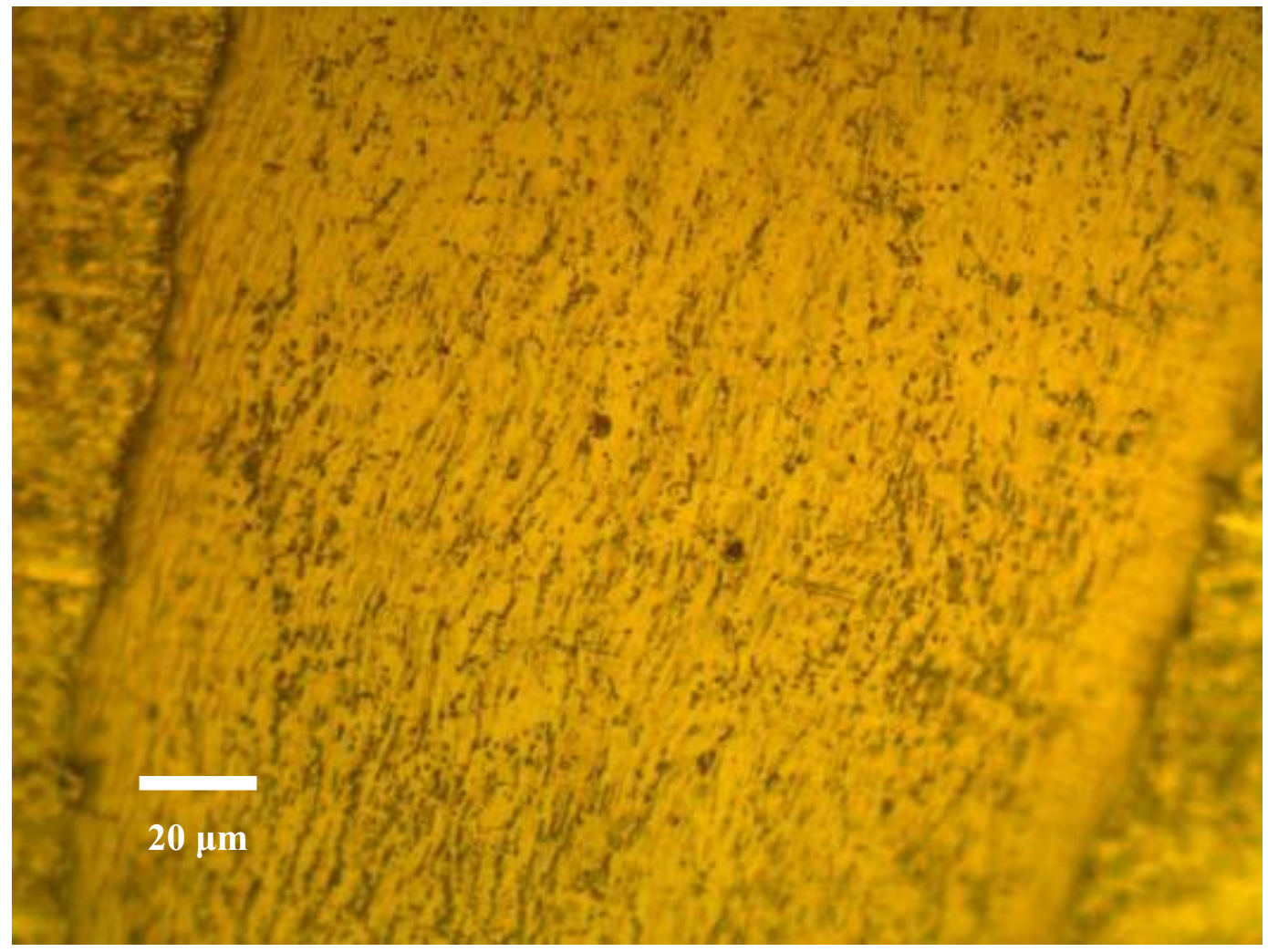

Figure D-2.e Bonded location near clip end of etched sample with DU10Mo foil in Al-6061 cladding, bonded by multiple-pass FB with aluminum-bronze tool face (500X photo).

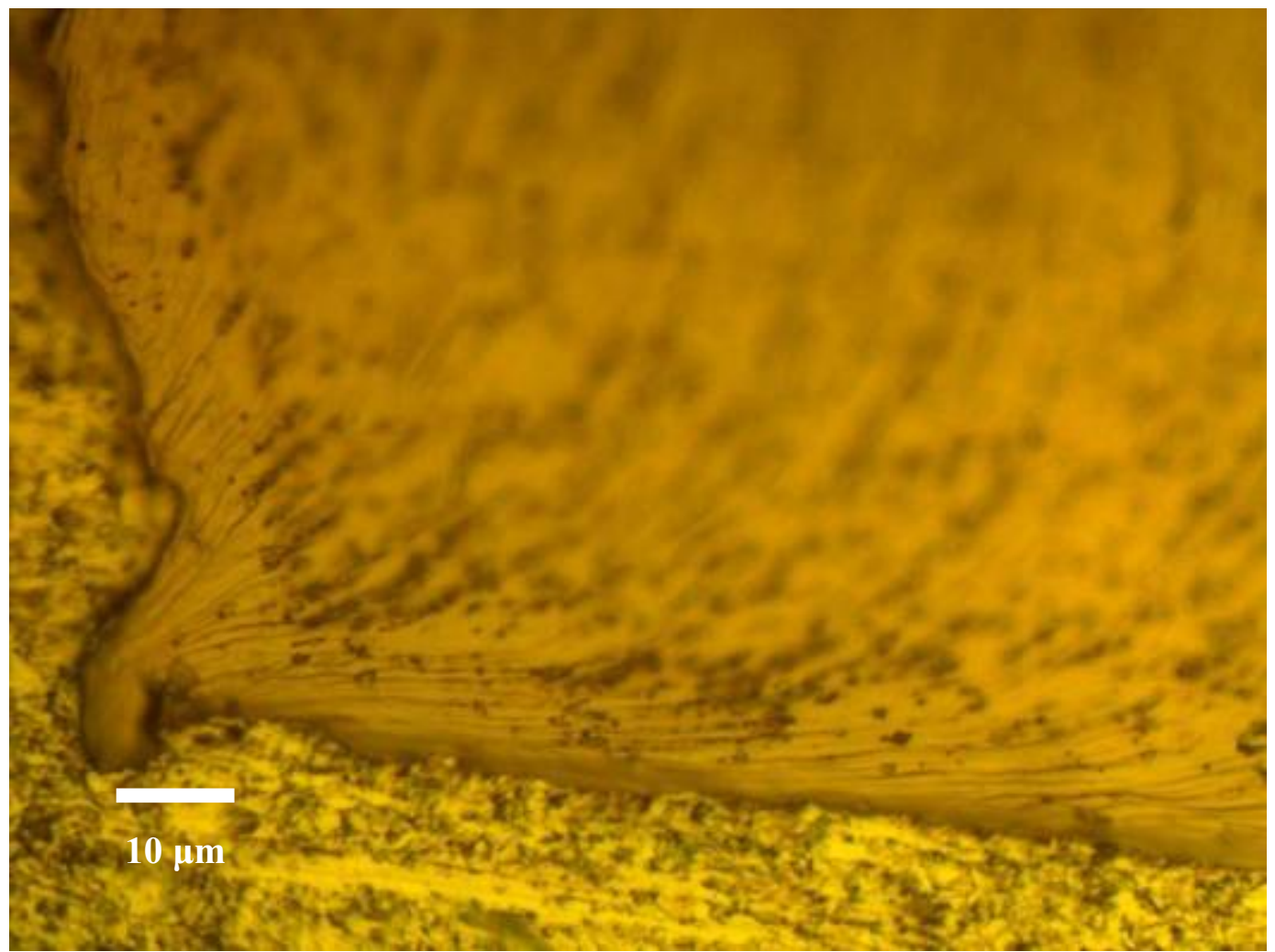

Figure D-2.f Bonded location near clip end of etched sample with DU10Mo foil in Al-6061 cladding, bonded by multiple-pass FB with aluminum-bronze tool face (1000X photo). 


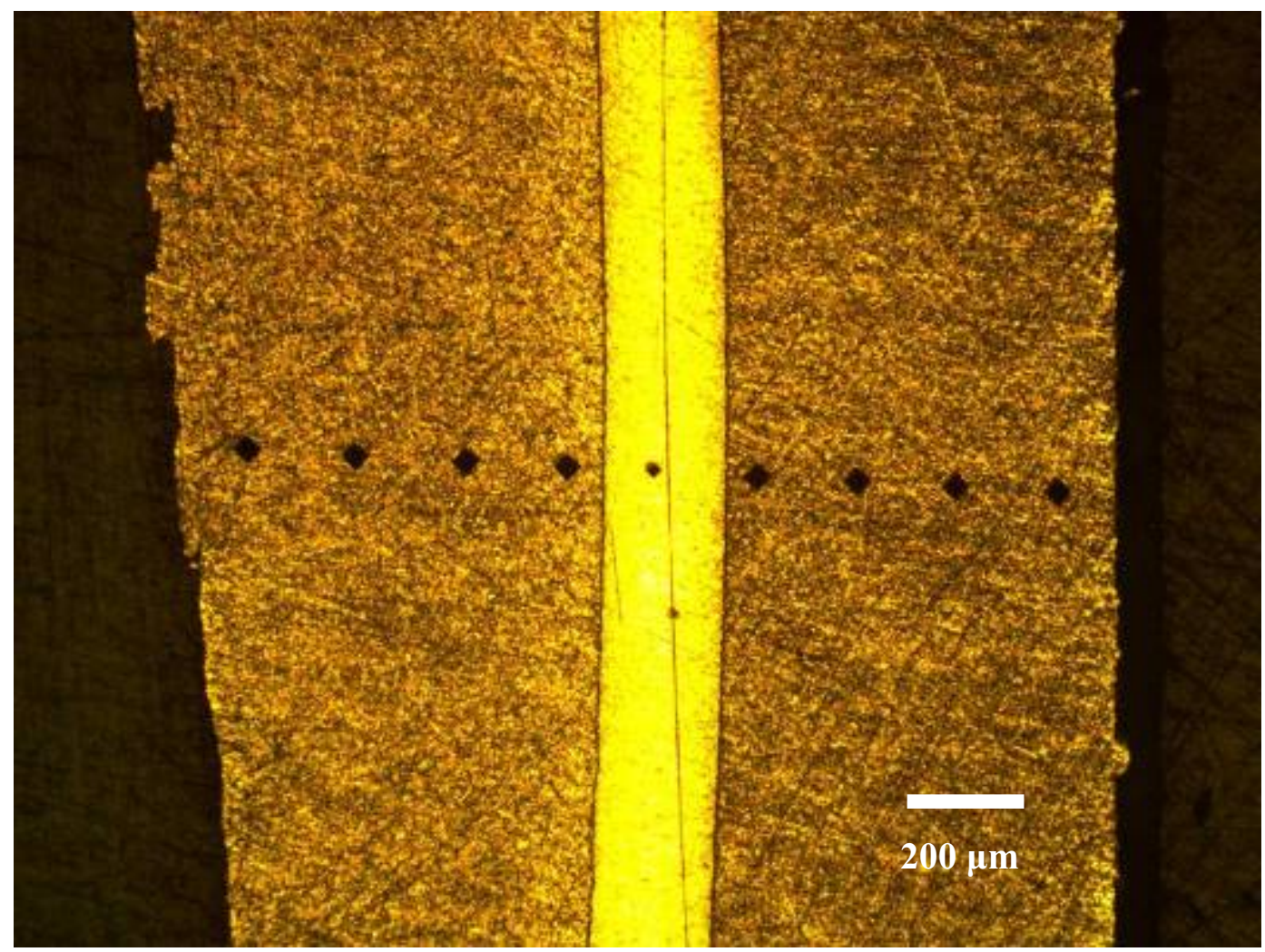

Figure D-2.g Another typical bonded location near foil center on sample bonded by multiple-pass FB with aluminum-bronze tool face, after etching was removed and hardness measurements taken (50X photo).

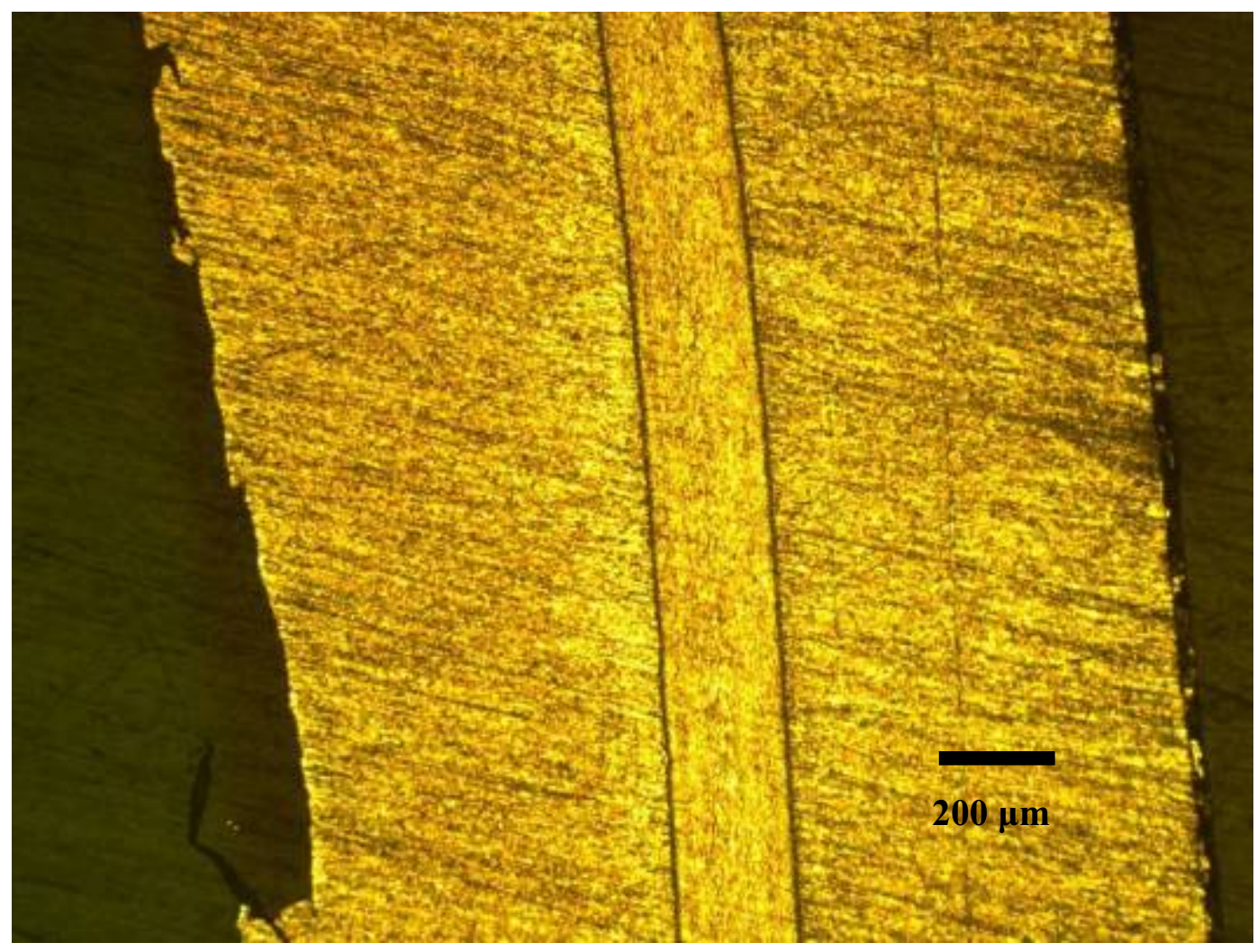

Figure D-2.h Same foil-center location on etched sample with DU10Mo foil in Al-6061 cladding, bonded by multiple-pass FB with aluminum-bronze tool face (50X photo). 


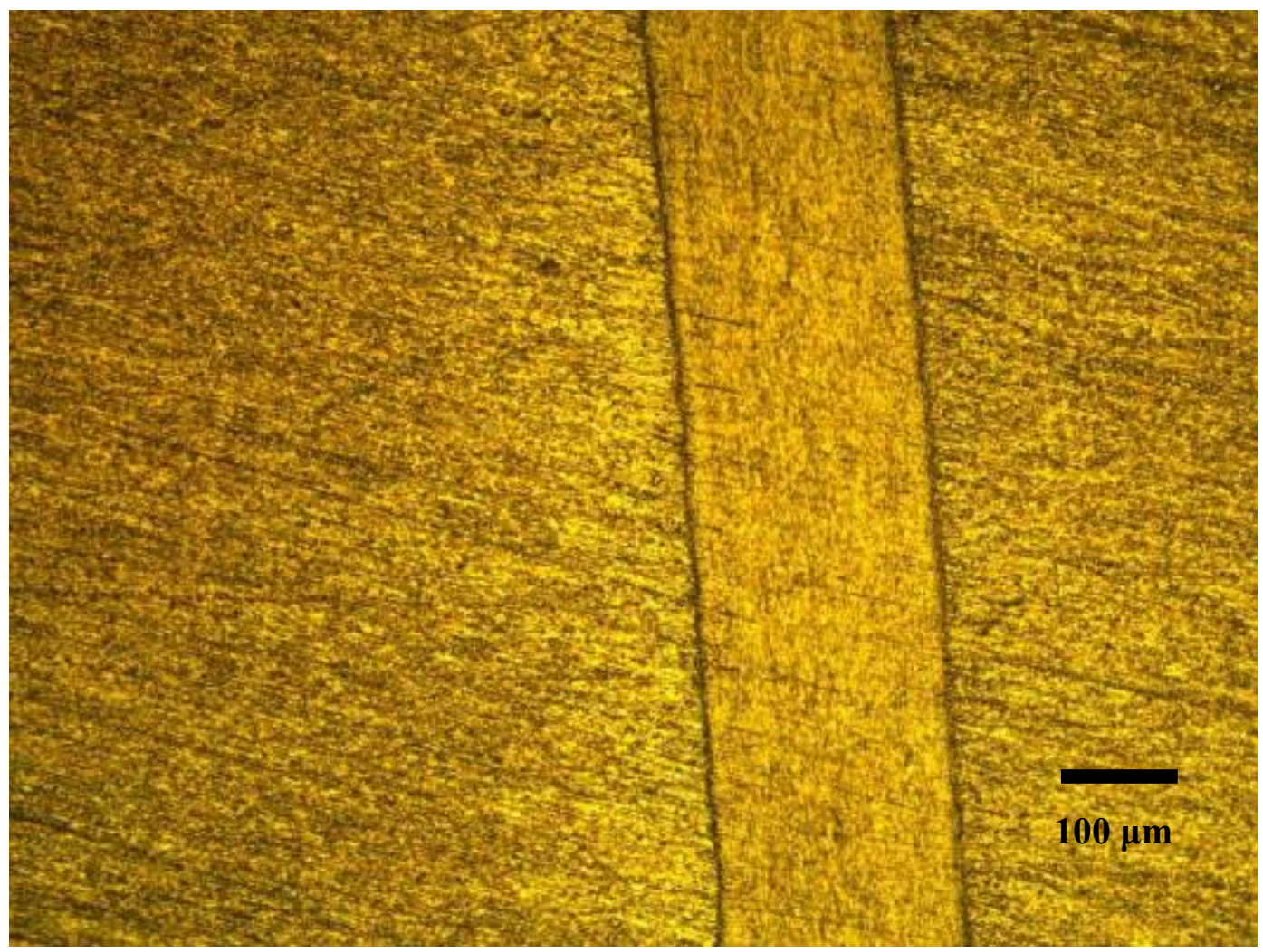

Figure D-2.i Same foil-center location on etched sample with DU10Mo foil in Al-6061 cladding, bonded by multiple-pass FB with aluminum-bronze tool face (100X photo).

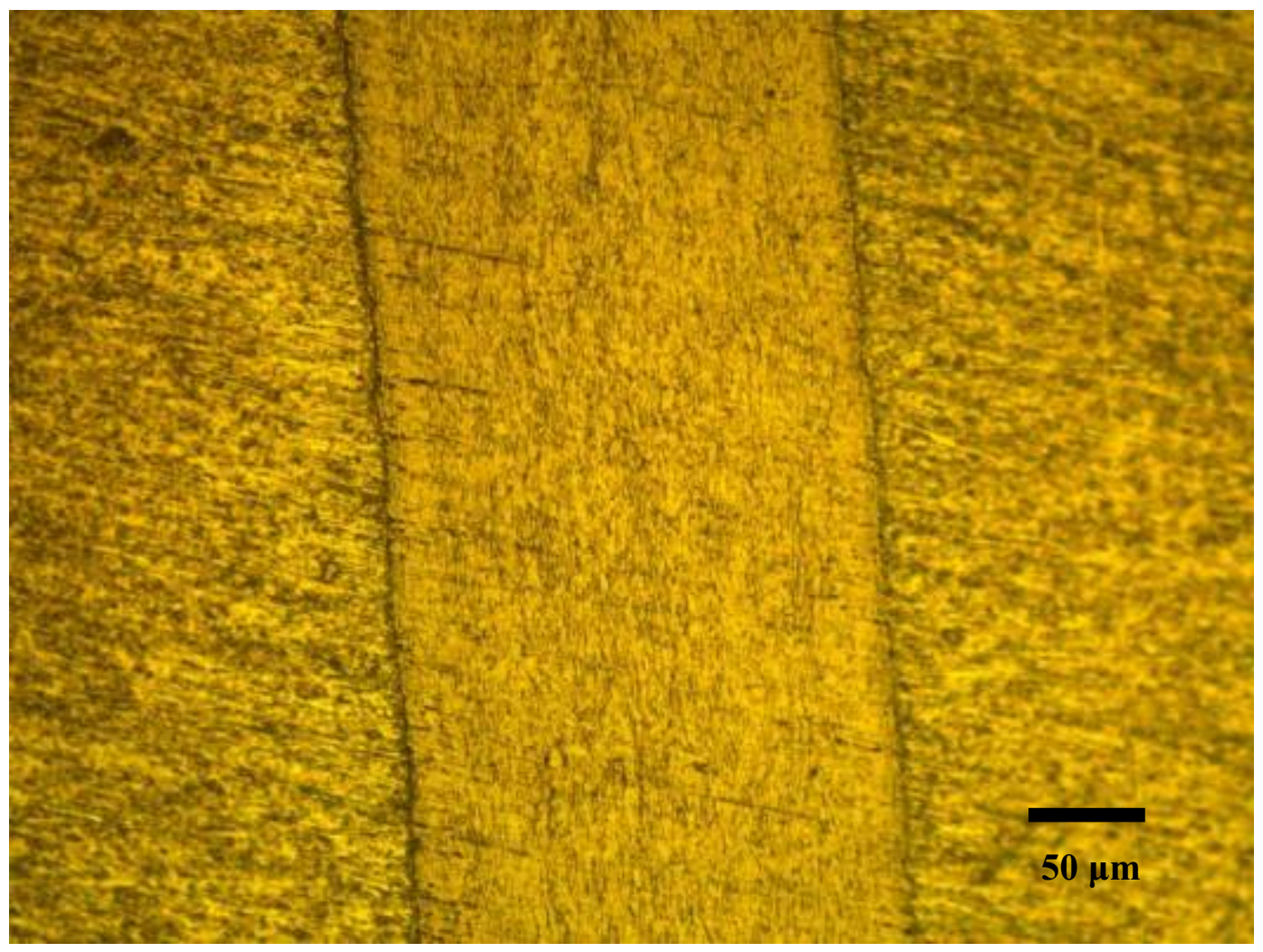

Figure D-2.j Same foil-center location on etched sample with DU10Mo foil in Al-6061 cladding, bonded by multiple-pass FB with aluminum-bronze tool face (200X photo). 

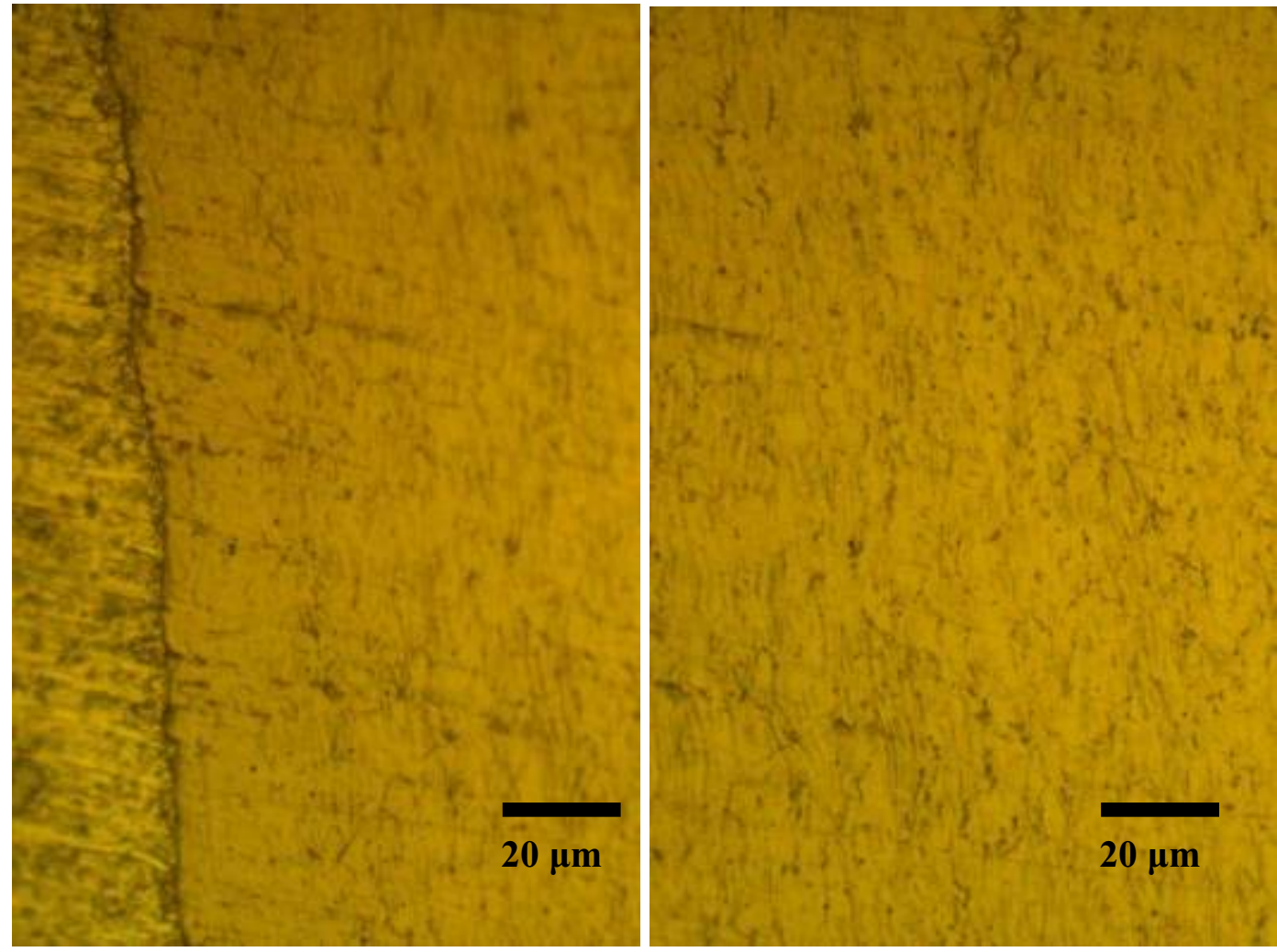

Figure D-2.k Same foil-center location on etched sample with DU10Mo foil in Al-6061 cladding, bonded by multiple-pass FB with aluminum-bronze tool face (500X photos): interface in focus (left), and U10Mo foil in focus (right).

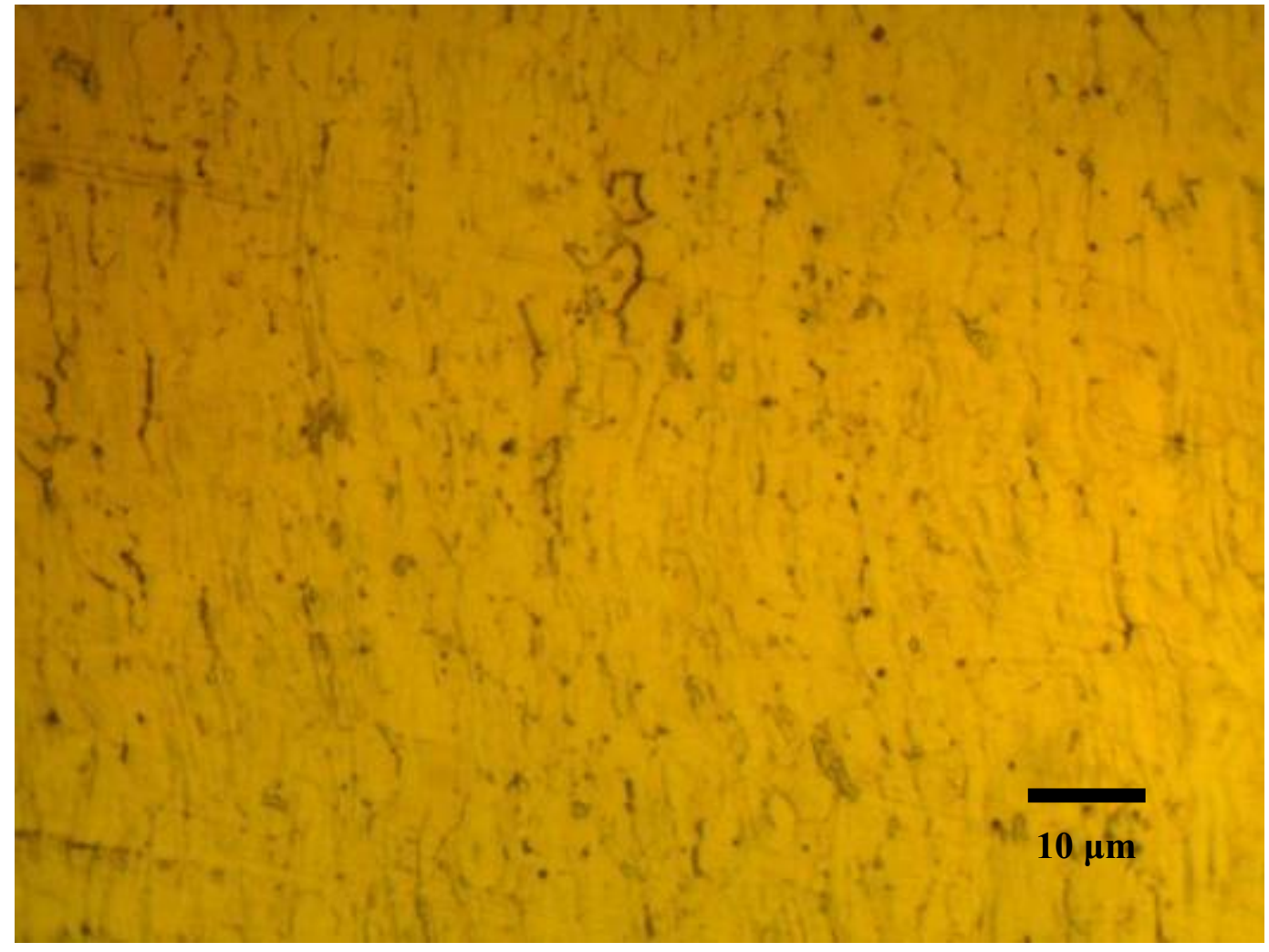

Figure D-2.l Same foil-center location on etched sample with DU10Mo foil in Al-6061 cladding, bonded by multiple-pass FB with aluminum-bronze tool face (1000X photo) 


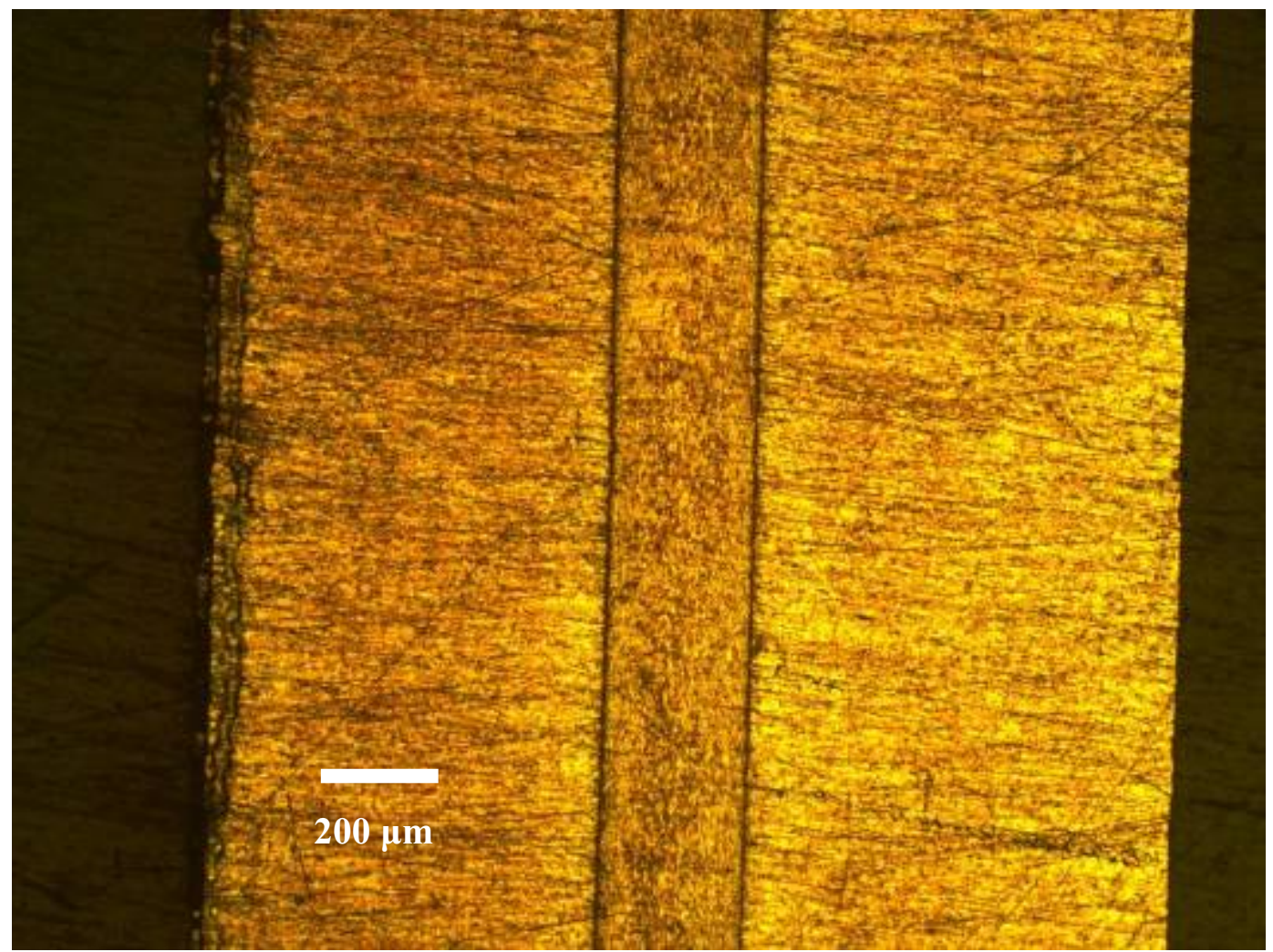

Figure D-3.a Typical location near clip end of etched sample with DU10Mo foil in Al-6061 cladding, bonded by multiple-pass FB with Anviloy tool face. Prior to re-polishing and hardness measurements (50X photo).

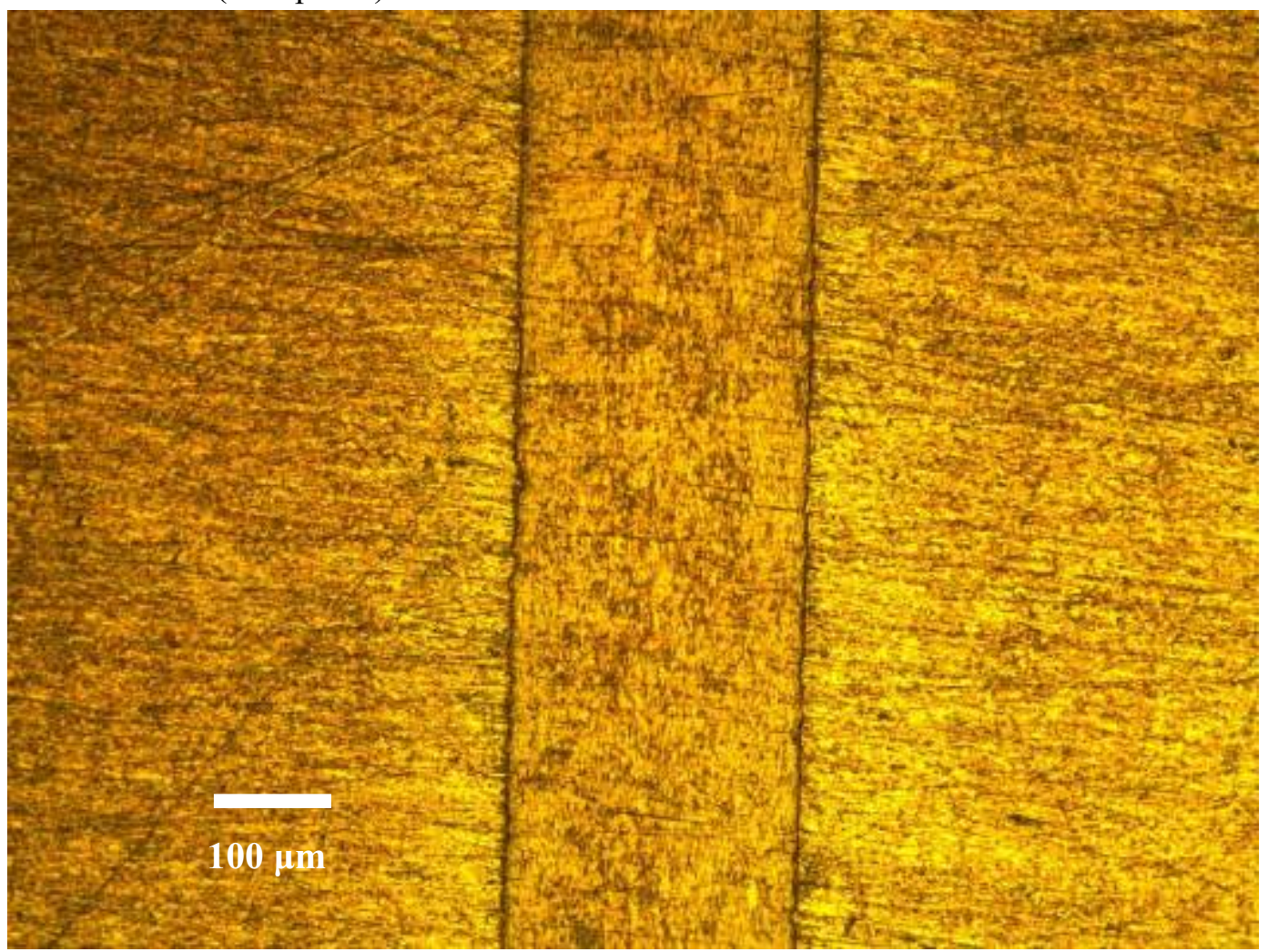

Figure D-3.b Same location near clip end of etched sample with DU10Mo foil in Al-6061 cladding, bonded by multiple-pass FB with Anviloy tool face (100X photo). 


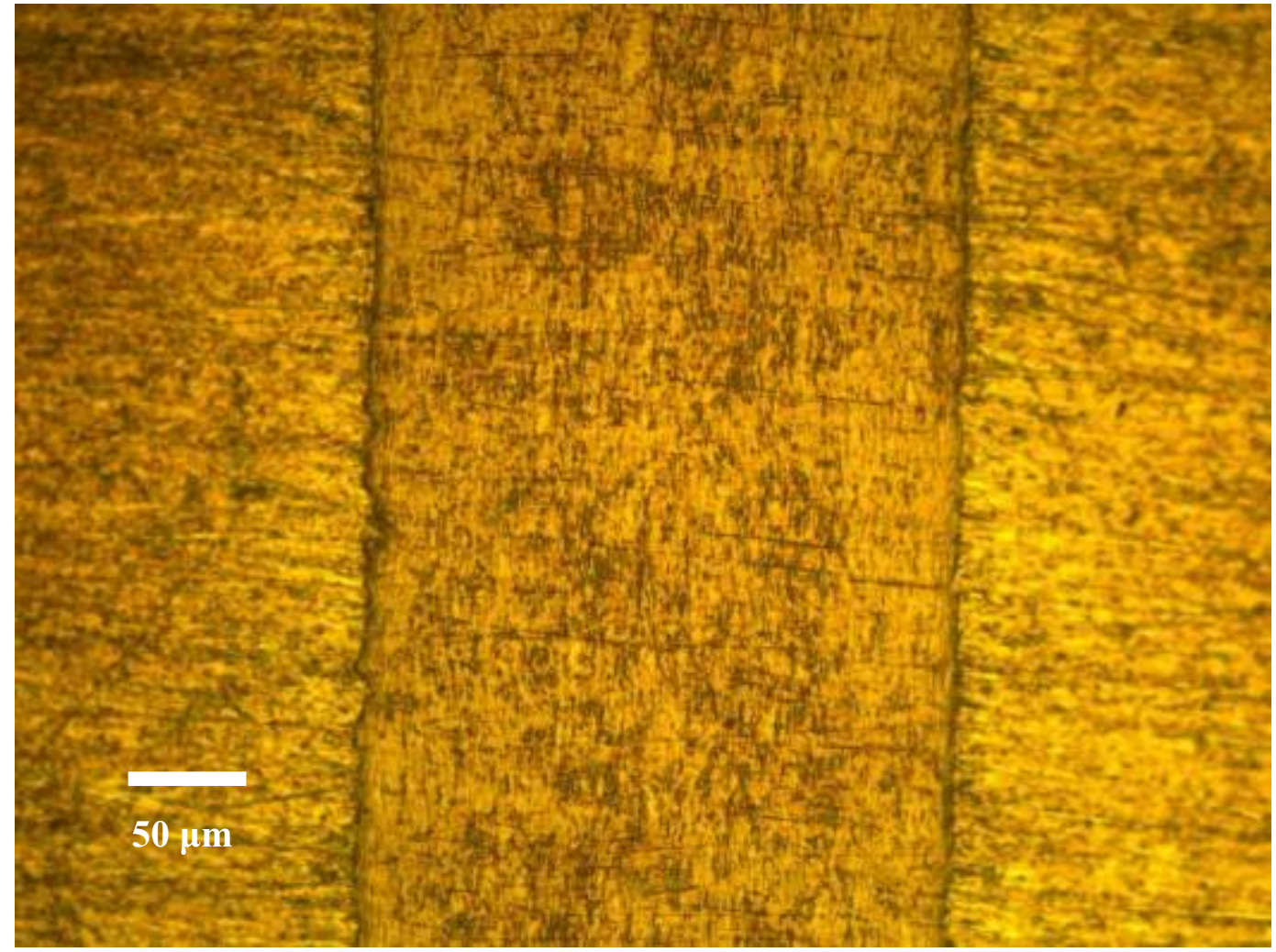

Figure D-3.c Same location near clip end of etched sample with DU10Mo foil in Al-6061 cladding, bonded by multiple-pass FB with Anviloy tool face (200X photo).

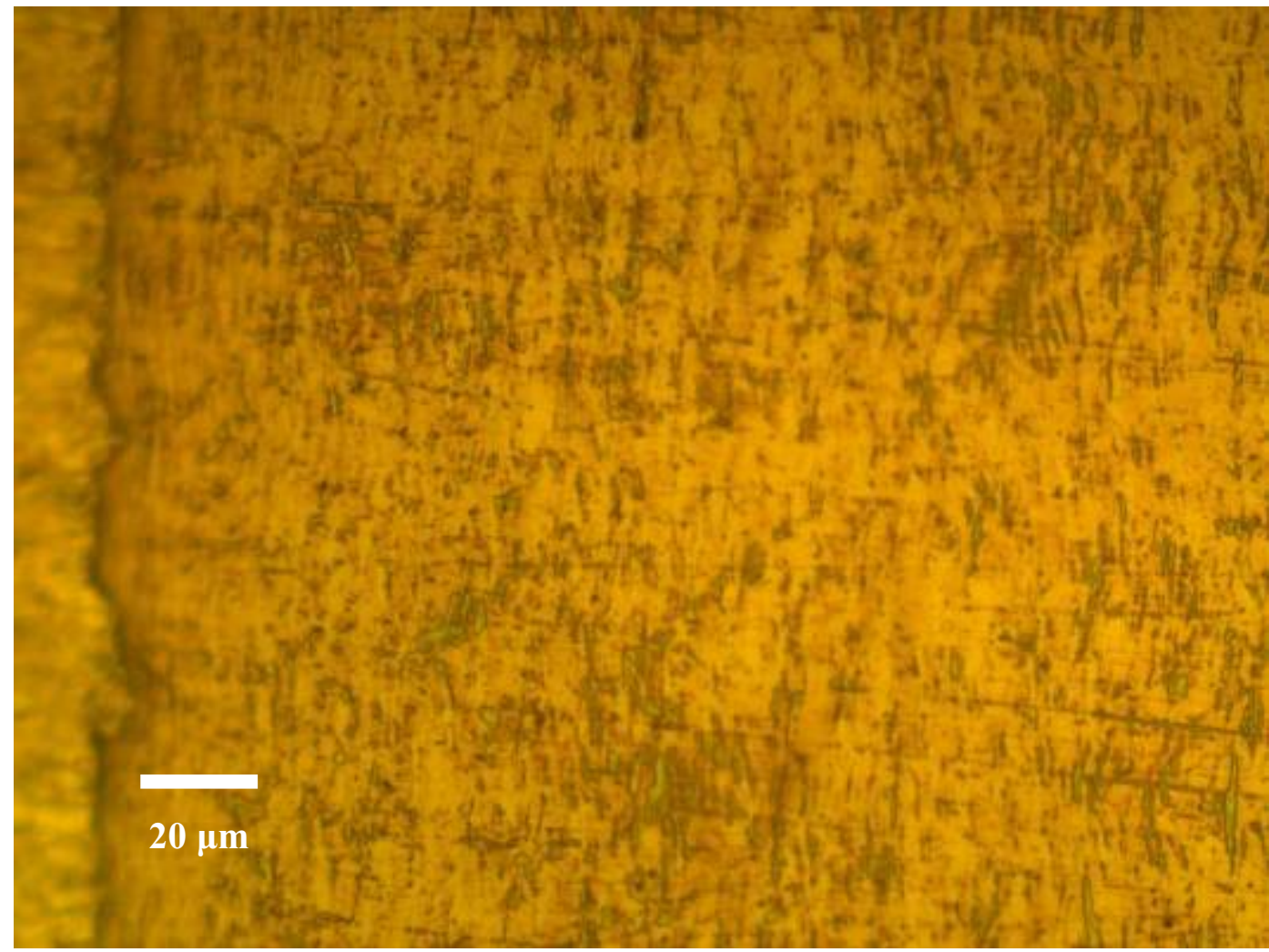

Figure D-3.d Same location near clip end of etched sample with DU10Mo foil in 6061 cladding, bonded by multiple-pass FB with Anviloy tool face (500X photo). 


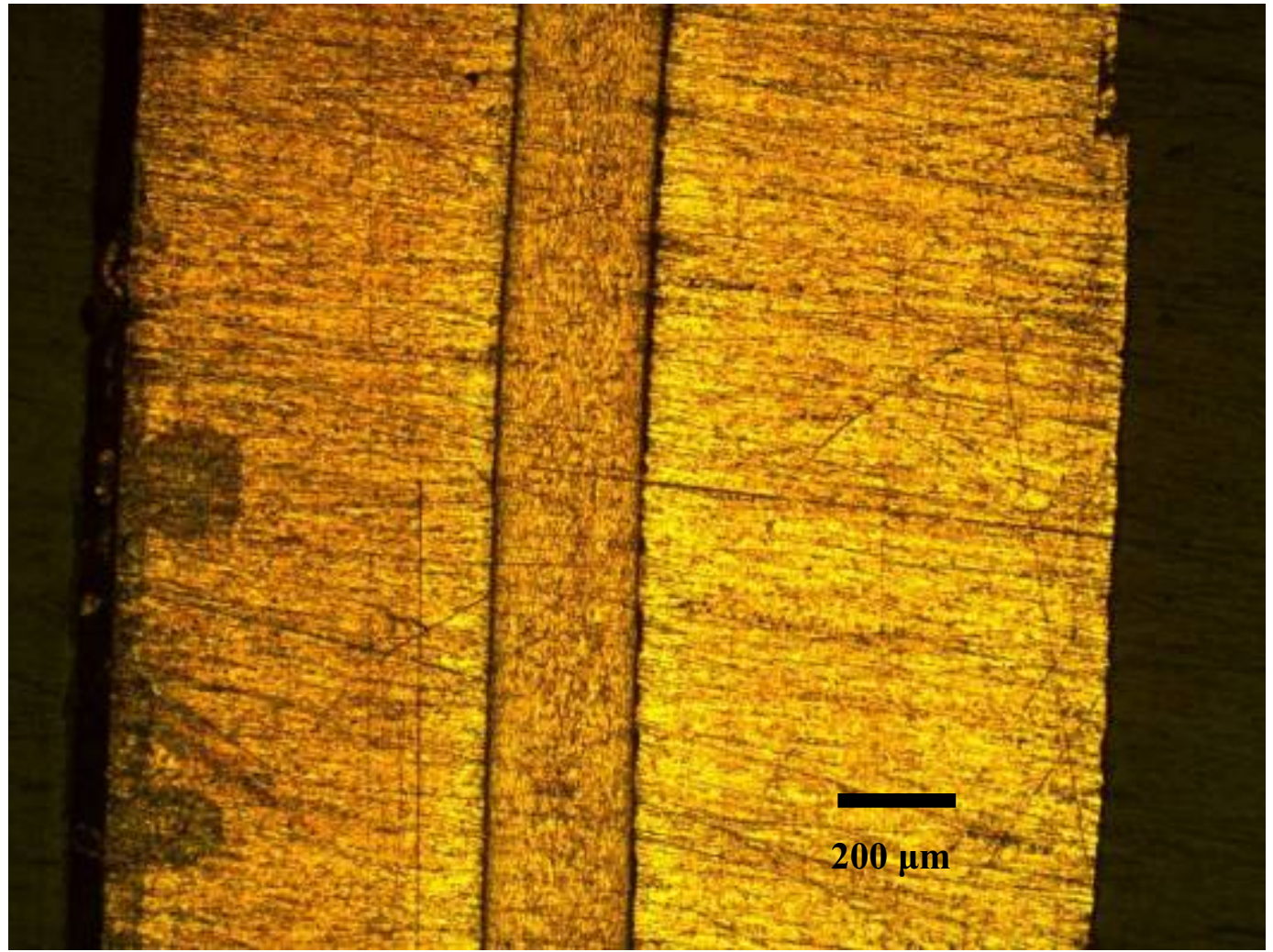

Figure D-3.e Location with most debond on etched sample with DU10Mo foil in Al6061 cladding, bonded by multiple-pass FB with Anviloy tool face (50X photo).

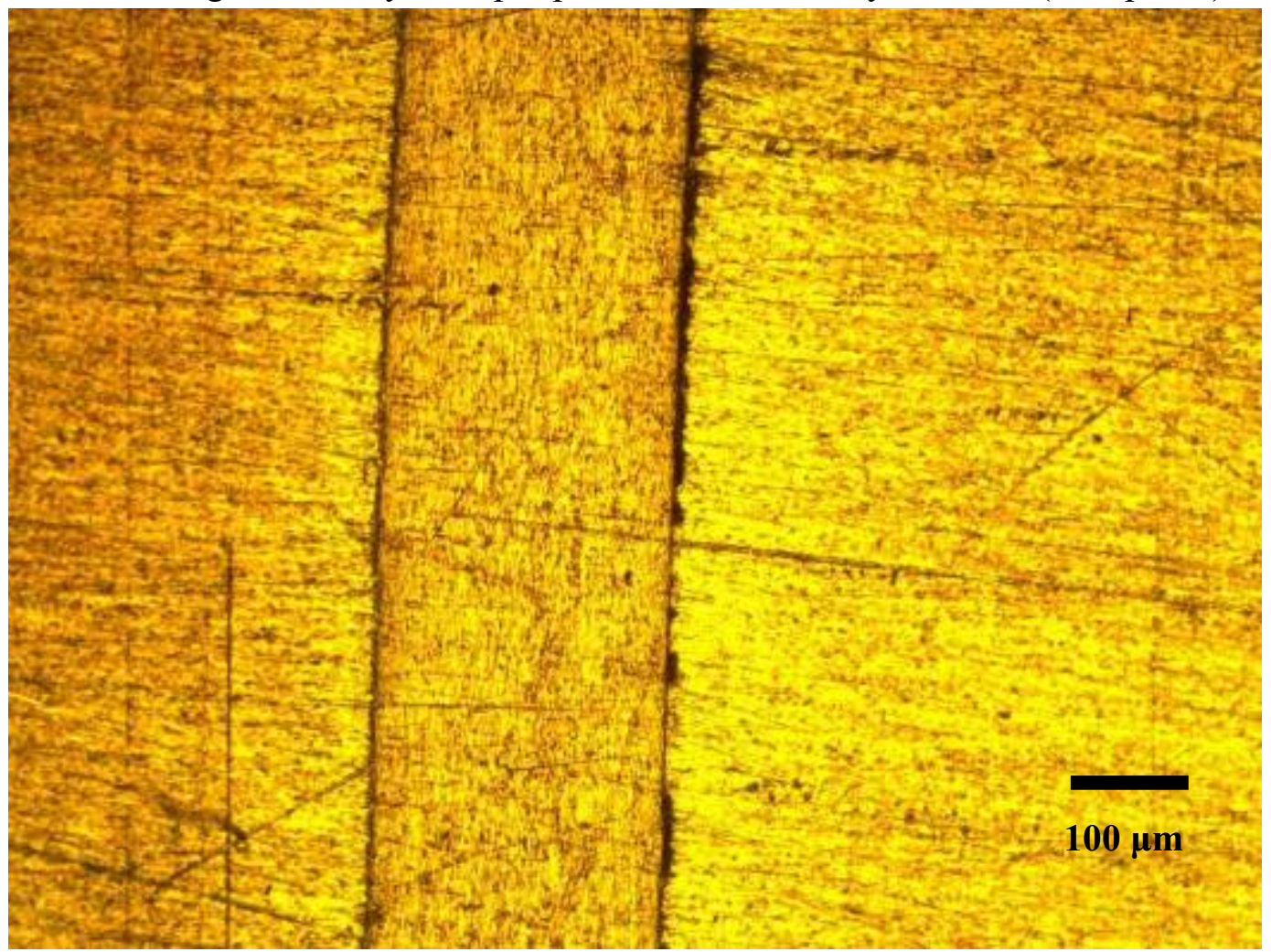

Figure D-3.f Same location with most debond, on etched sample with DU10Mo foil in Al6061 cladding, bonded by multiple-pass FB with Anviloy tool face (100X photo). 


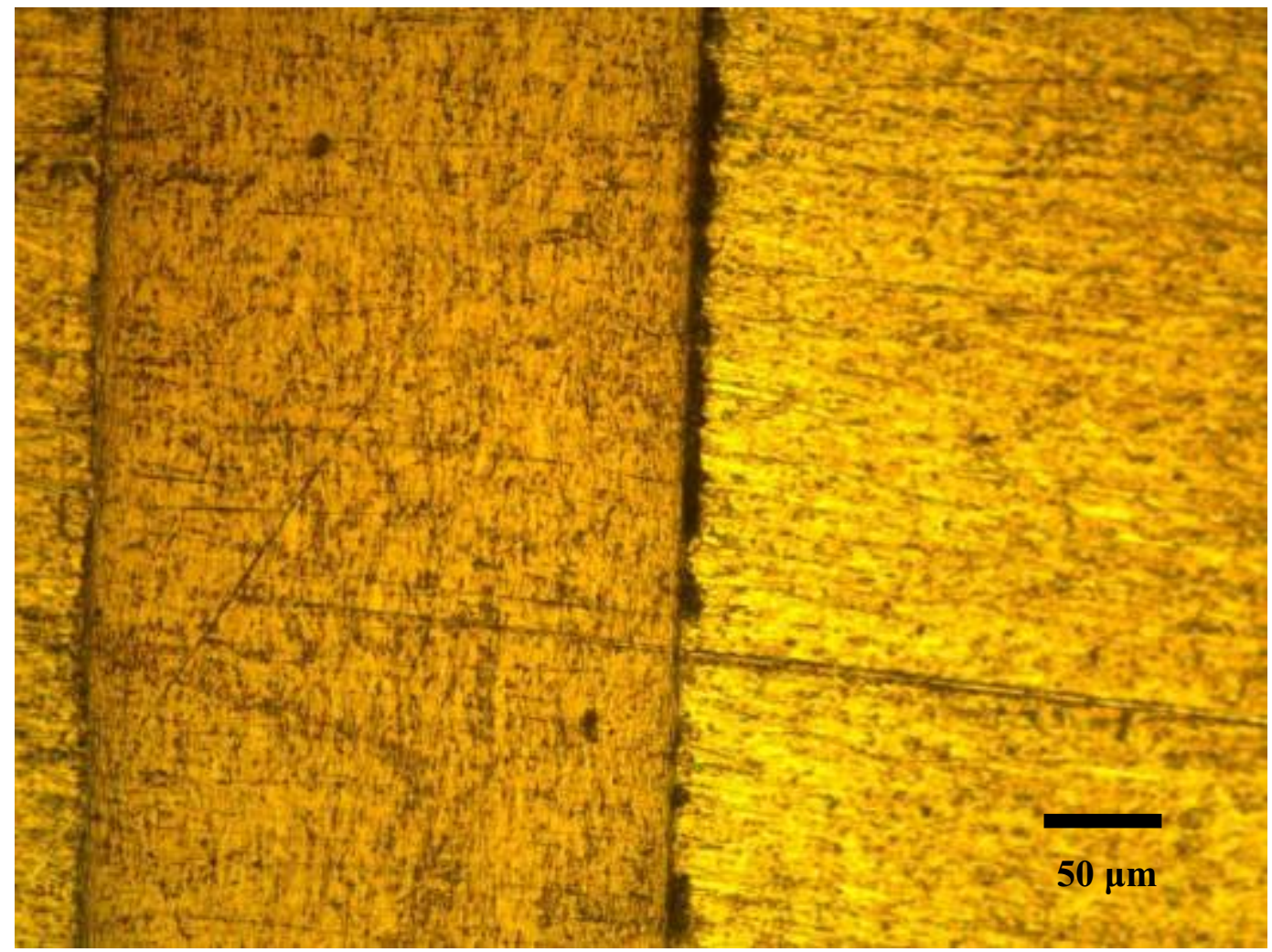

Figure D-3.g Same location with most debond, on etched sample with DU10Mo foil in A16061 cladding, bonded by multiple-pass FB with Anviloy tool face (200X photo).

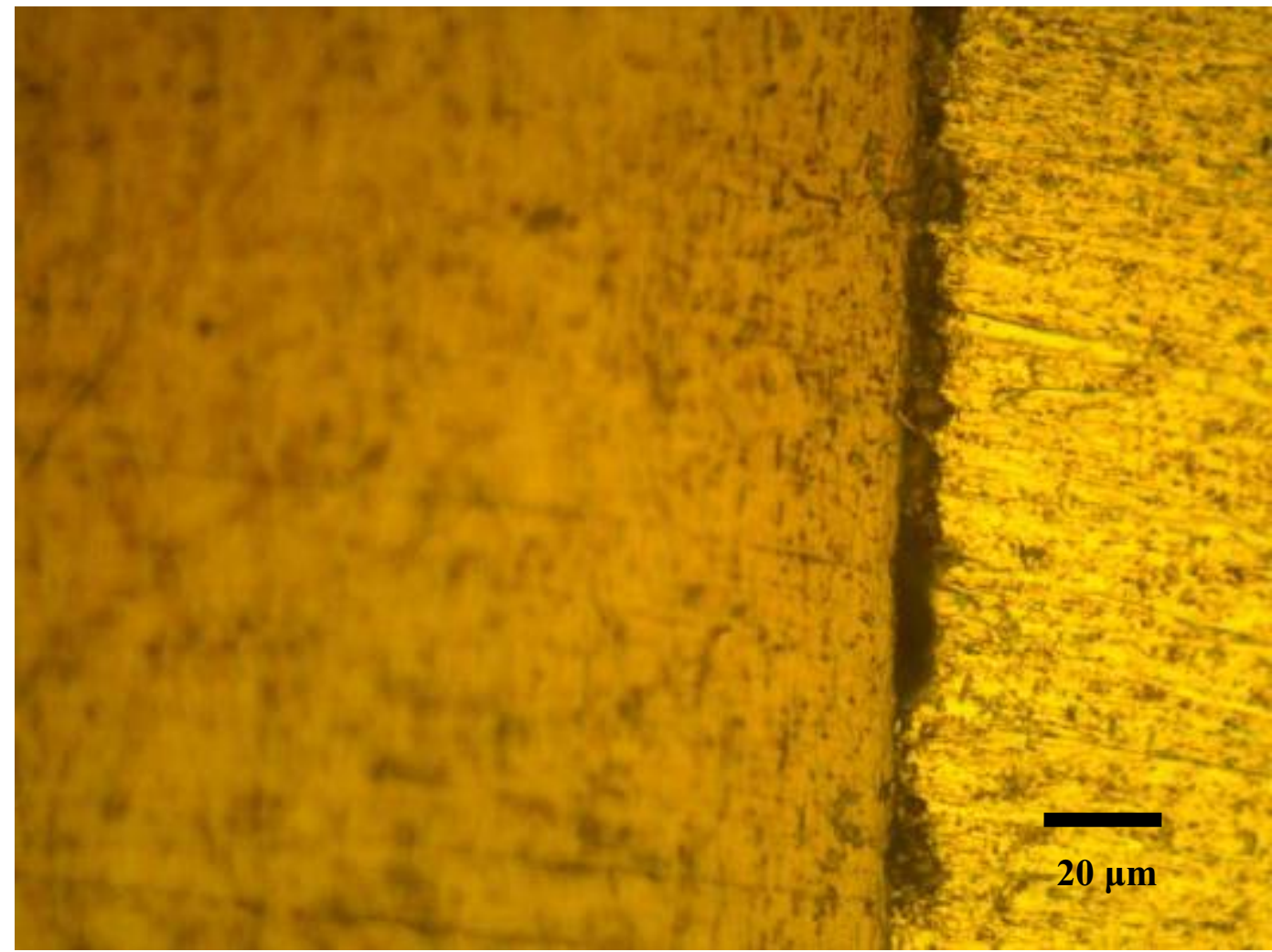

Figure D-3.h Same location with most debond, on etched sample with DU10Mo foil in Al6061 cladding, bonded by multiple-pass FB with Anviloy tool face (500X photo). 


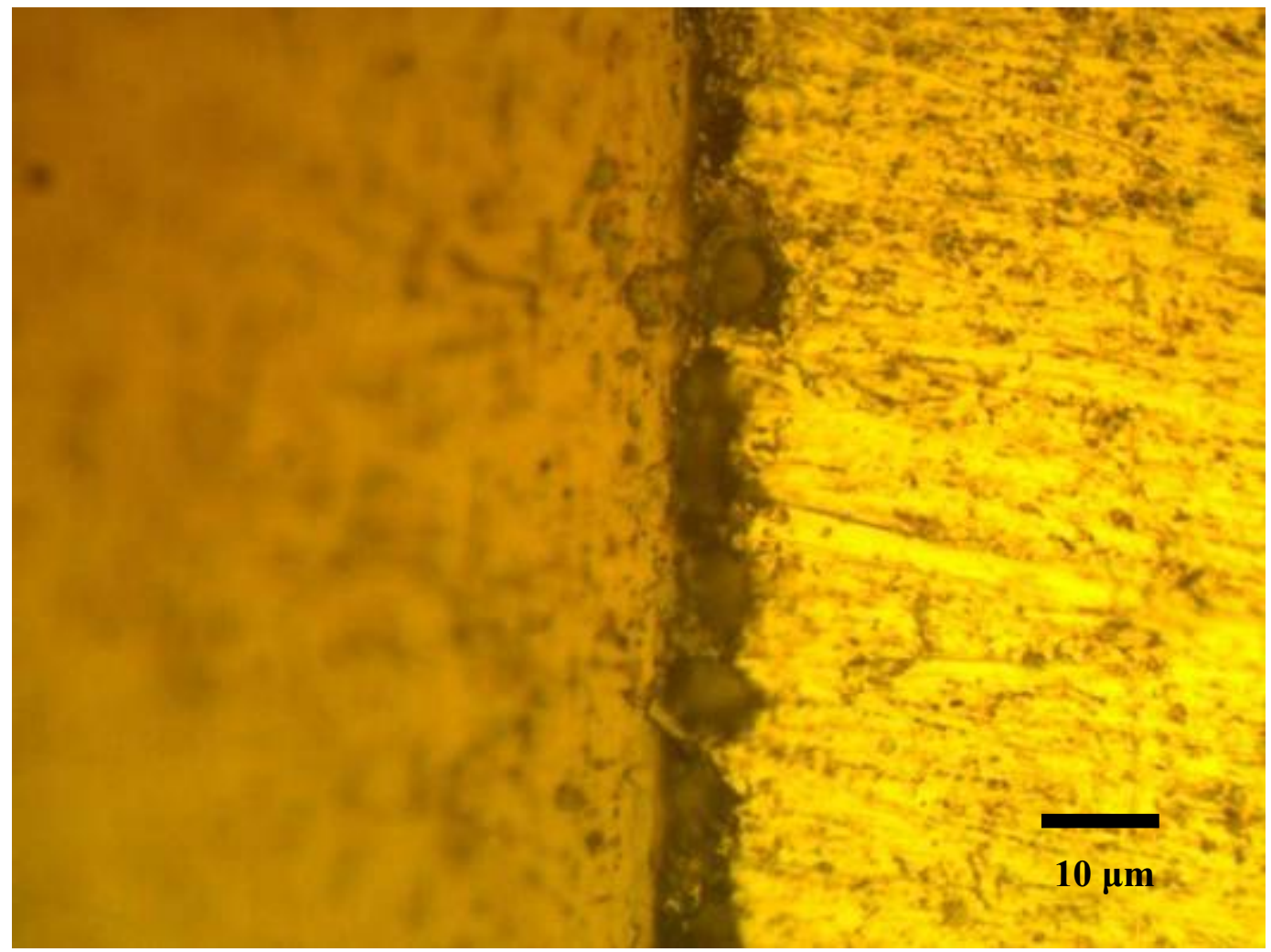

Figure D-3.i Same location with most debond, on etched sample with DU10Mo foil in A16061 cladding, bonded by multiple-pass FB with Anviloy tool face (1000X photo). 
APPENDIX D

Microphotos of DU10Mo Monolithic Fuel Plate Samples

(page is intentionally blank) 


\section{Microstructural Examination to Aid in Understanding Friction Bonding Fabrication Technique for Monolithic Nuclear Fuel}

\section{APPENDIX E \\ Friction Bonding Sample Hardness Measurements with Statistics}

This appendix provides some statistical information on hardness measurements* described and evaluated in the report main text. Tables E-1 through E-7 give two statistics for hardness measurements taken at each aluminum location: (1) The standard error, $\varepsilon$, was calculated and reported by the measurement software ("ConfiDent"), based on the hardness measurement numbers internal to the software calculation, i.e., with more significant digits than reported by the software and available to give here; (2) the aluminum measurements' standard deviations were then calculated based on $\varepsilon$ rather than on the listed hardness values, to take advantage of the additional accuracy inherent in the $\varepsilon$ number. Table E- 8 gives standard deviations for hardness measurements on the DU10Mo sample foils. These were calculated based on the listed hardness values rather than on $\varepsilon$, which was not available from the ConfiDent software since the foils' hardness measurements were taken at different times and not processed by the software as comprehensive sample sets.

Calculations were performed as follows:

$\underline{\text { Standard Error }}=\varepsilon$, sometimes termed the "Standard Deviation of the Mean," indicates the standard deviation, not of the measured hardness values, but of the difference between those values and the mean. Where $\sigma=$ standard deviation of a population, then Standard Error $=\varepsilon=$ $\sigma / \operatorname{SQRT}(n)=\left[\operatorname{SQRT}\left(\left(\Sigma\left(\mathrm{Avg}-\mathrm{H}_{\mathrm{V}}\right)^{2}\right) / n\right)\right] / \operatorname{SQRT}(n)$. Number $\varepsilon$ in the following tables was reported by ConfiDent software, calculated based on sample size $n$.

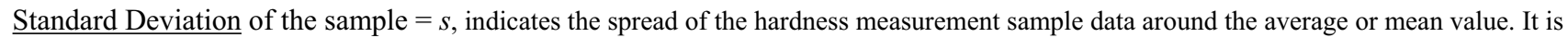
calculated in these tables as Standard Deviation $=s=\operatorname{SQRT}\left(\left(\Sigma\left(\text { Avg- } \mathrm{H}_{\mathrm{V}}\right)^{2}\right) /(n-1)\right)=(\sigma n) /(n-1)=[\varepsilon \operatorname{SQRT}(n)] n /(n-1)$. It is calculated in Tables E-1 through E-7 based on $\varepsilon$ rather than on the hardness values listed there, to take advantage of the additional accuracy inherent in the $\varepsilon$ number. However, standard deviations given in Table E- 8 are based on the listed hardness values, as values of $\varepsilon$ were not available.

* Hardness measurements were performed per ASTM E384-06 (2006). "Standard test method for microindentation hardness of materials." West Conshohocken, USA: ASTM, 2006. The measurements were performed in the EML using the LECO Hardness Tester, with ConfiDent Software Version 2.5.1 by LECO (c)1998-2002, in conjunction with a Dell PC and a LECO Corp. Model LM-100 hardness tester and optical microscope. (Set at default conditions of 640x480 source, 50X objective, and 100gf load for Vickers indentor). 


\section{APPENDIX E - Friction Bonding Sample Hardness Measurements with Statistics}

\section{$\underline{\text { Tables }}$}

E-1 Vickers Hardness $H_{V}$ measurements across " $8 \mathrm{~K}$ " aluminum sample fabricated with $35.6 \mathrm{kN}$ (8,000 lbf) FB tool load

E-2 Vickers Hardness $H_{V}$ measurements across "10K" aluminum sample fabricated with $44.5 \mathrm{kN}$ (10,000 lbf) FB tool load

E-3 Vickers Hardness $H_{V}$ measurements across " $12 \mathrm{~K}$ ” aluminum sample fabricated with $53.4 \mathrm{kN}$ (12,000 lbf) FB tool load

E-4 Vickers Hardness $H_{V}$ measurements across " $14 \mathrm{~K}$ " aluminum sample fabricated with $62.3 \mathrm{kN}$ (14,000 lbf) FB tool load

E-5 Vickers Hardness $H_{V}$ measurements across aluminum in DU10Mo sample fabricated with Steel-A36 FB tool face

E-6 Vickers Hardness $\mathrm{H}_{\mathrm{V}}$ measurements across aluminum in DU10Mo sample fabricated with Al-Bronze FB tool face

E-7 Vickers Hardness $\mathrm{H}_{\mathrm{V}}$ measurements across aluminum in DU10Mo sample fabricated with Anviloy FB tool face

E-8 Vickers Hardness $\mathrm{H}_{\mathrm{V}}$ measurements along foils in DU10Mo samples fabricated with varying FB tool face alloys

Table E-1. Vickers Hardness $\mathrm{H}_{\mathrm{V}}$ measurements across " $8 \mathrm{~K}$ " aluminum sample fabricated with $35.6 \mathrm{kN}$ (8,000 lbf) FB tool load.

\begin{tabular}{|c|c|c|c|c|c|c|c|c|c|c|c|c|}
\hline Location & $\frac{\text { FB Side }}{1}$ & 2 & 3 & 4 & 5 & 6 & 7 & $\frac{\text { Non-FB }}{\frac{\text { Side }}{8}}$ & $\begin{array}{l}\text { Average } \\
\text { ("Mean") }\end{array}$ & $\begin{array}{c}\text { Standard } \\
\text { Error } \\
\varepsilon^{\mathrm{a}}\end{array}$ & $\begin{array}{c}\text { Standard } \\
\text { Deviation } \\
s^{\text {a }}\end{array}$ & Date \\
\hline $\begin{array}{l}\text { Advancing } \\
\text { Edge }\end{array}$ & 73 & 66 & 68 & 74 & 77 & 75 & 74 & 75 & 72.8 & 1.25 & 4.04 & $5-29-07$ \\
\hline Center & 69 & 69 & 70 & 69 & 72 & 73 & 72 & 71 & 70.6 & 0.5449 & 1.76 & $5-22-07$ \\
\hline $\begin{array}{l}\text { Retreating } \\
\text { Edge }\end{array}$ & 64 & 62 & 70 & 73 & 70 & 75 & 72 & 74 & 70.0 & 1.5512 & 5.01 & $5-29-07$ \\
\hline
\end{tabular}

a. Standard Error $\varepsilon$ was reported by ConfiDent software, and used here to calculate the Standard Deviation, $s$. 
APPENDIX E - Friction Bonding Sample Hardness Measurements with Statistics

Table E-2. Vickers Hardness $H_{V}$ measurements across “10K” aluminum sample fabricated with $44.5 \mathrm{kN}$ (10,000 lbf) FB tool load.

\begin{tabular}{|c|c|c|c|c|c|c|c|c|c|c|c|c|}
\hline Location & $\frac{\text { FB Side }}{1}$ & 2 & 3 & 4 & 5 & 6 & 7 & $\frac{\text { Non-FB }}{\frac{\text { Side }}{8}}$ & $\begin{array}{c}\text { Average } \\
\text { ("Mean") }\end{array}$ & $\begin{array}{c}\text { Standard } \\
\text { Error } \\
\varepsilon^{\mathrm{a}} \\
\end{array}$ & $\begin{array}{c}\text { Standard } \\
\text { Deviation } \\
s^{\mathrm{a}} \\
\end{array}$ & Date \\
\hline $\begin{array}{l}\text { Advancing } \\
\text { Edge }\end{array}$ & 71 & 68 & 74 & 70 & 67 & 71 & 70 & 71 & 70.3 & 0.7071 & 2.29 & $6-11-07$ \\
\hline Center $^{b}$ & 75 & 72 & 72 & 72 & 78 & 76 & 79 & & 74.9 & 1.04 & 3.36 & 5-29-07 \\
\hline "A" & 70 & 72 & 70 & 73 & 78 & 76 & 74 & 74 & 73.4 & 0.927 & 3.00 & $5-23-07$ \\
\hline $\begin{array}{l}\text { Retreating } \\
\text { Edge }\end{array}$ & 60 & 67 & 70 & 72 & 77 & 74 & 73 & 73 & 60 & 1.7321 & 5.60 & 6-11-07 \\
\hline
\end{tabular}

a. Standard Error $\varepsilon$ was reported by ConfiDent software, and used here to calculate the Standard Deviation, $s$.

b. The "Center" location on this sample had only 7 hardness measurements taken across its full thickness: $n=7$ for this location.

Table E-3. Vickers Hardness $H_{V}$ measurements across "12K" aluminum sample fabricated with 53.4 kN (12,000 lbf) FB tool load.

\begin{tabular}{|c|c|c|c|c|c|c|c|c|c|c|c|c|}
\hline Location & FB Side & 2 & 3 & 4 & 5 & 6 & 7 & $\frac{\text { Non-FB }}{\frac{\text { Side }}{8}}$ & $\begin{array}{l}\text { Average } \\
\text { ("Mean") }\end{array}$ & $\begin{array}{c}\text { Standard } \\
\text { Error } \\
\varepsilon^{\mathrm{a}} \\
\end{array}$ & $\begin{array}{c}\text { Standard } \\
\text { Deviation } \\
s^{\text {a }} \\
\end{array}$ & Date \\
\hline $\begin{array}{l}\text { Advancing } \\
\text { Edge }\end{array}$ & 12 & 73 & 75 & 72 & 70 & 70 & 74 & 70 & 73 & 1.1319 & 3.66 & $6-11-07$ \\
\hline Center & 77 & 71 & 74 & 72 & 74 & 71 & 73 & 71 & 72.9 & 0.696 & 2.25 & 5-23-07 \\
\hline $\begin{array}{l}\text { Retreating } \\
\text { Edge }\end{array}$ & 70 & 70 & 72 & 68 & 72 & 71 & 71 & 72 & 70.8 & 0.4677 & 1.51 & 6-11-07 \\
\hline
\end{tabular}

a. Standard Error $\varepsilon$ was reported by ConfiDent software, and used here to calculate the Standard Deviation, $s$. 
APPENDIX E - Friction Bonding Sample Hardness Measurements with Statistics

Table E-4. Vickers Hardness $H_{V}$ measurements across "14K" aluminum sample fabricated with $62.3 \mathrm{kN}$ (14,000 lbf) FB tool load.

\begin{tabular}{|c|c|c|c|c|c|c|c|c|c|c|c|c|}
\hline Location & $\frac{\text { FB Side }}{1}$ & 2 & 3 & 4 & 5 & 6 & 7 & $\frac{\text { Non-FB }}{\frac{\text { Side }}{8}}$ & $\begin{array}{l}\text { Average } \\
\text { ("Mean") }\end{array}$ & $\begin{array}{c}\text { Standard } \\
\text { Error } \\
\varepsilon^{\mathrm{a}} \\
\end{array}$ & $\begin{array}{c}\text { Standard } \\
\text { Deviation } \\
s^{\mathrm{a}} \\
\end{array}$ & Date \\
\hline $\begin{array}{l}\text { Advancing } \\
\text { Edge }\end{array}$ & 79 & 76 & 76 & 78 & 78 & 78 & 78 & 78 & 77.6 & 0.515 & 1.66 & $6-13-07$ \\
\hline Center & 71 & 72 & 73 & 77 & 77 & 77 & 75 & 74 & 74.5 & 0.8101 & 2.62 & $5-22-07$ \\
\hline $\begin{array}{l}\text { Retreating } \\
\text { Edge }\end{array}$ & 66 & 71 & 71 & 73 & 74 & 72 & 72 & 74 & 71.6 & 0.857 & 2.77 & $6-13-07$ \\
\hline
\end{tabular}

a. Standard Error $\varepsilon$ was reported by ConfiDent software, and used here to calculate the Standard Deviation, $s$.

Table E-5. Vickers Hardness $H_{V}$ measurements across aluminum in DU10Mo sample fabricated with Steel-A36 FB tool face.

\begin{tabular}{|c|c|c|c|c|c|c|c|c|c|c|c|c|}
\hline Location & $\underline{\text { Side } 1}$ & 2 & 3 & 4 & 5 & 6 & 7 & $\frac{\text { Side } 2}{8}$ & $\begin{array}{c}\text { Average } \\
\text { ("Mean") }\end{array}$ & $\begin{array}{c}\text { Standard } \\
\text { Error } \\
\varepsilon^{\mathrm{a}} \\
\end{array}$ & $\begin{array}{c}\text { Standard } \\
\text { Deviation } \\
s^{\mathrm{a}} \\
\end{array}$ & Date \\
\hline Top & 68 & 74 & 80 & 71 & 77 & 81 & 80 & 83 & 76.8 & 1.75 & 5.66 & $10-3-07$ \\
\hline Center & 75 & 71 & 72 & 72 & 75 & 80 & 78 & 82 & 75.6 & 1.3405 & 4.33 & $10-3-07$ \\
\hline Clip End & 71 & 74 & 76 & 75 & 73 & 75 & 81 & 83 & 76 & 1.3346 & 4.31 & $10-3-07$ \\
\hline
\end{tabular}

a. Standard Error $\varepsilon$ was reported by ConfiDent software, and used here to calculate the Standard Deviation, $s$. 
APPENDIX E - Friction Bonding Sample Hardness Measurements with Statistics

Table E-6. Vickers Hardness $\mathrm{H}_{\mathrm{V}}$ measurements across aluminum in DU10Mo sample fabricated with Aluminum-Bronze FB tool face.

\begin{tabular}{|c|c|c|c|c|c|c|c|c|c|c|c|c|}
\hline Location & $\underline{\text { Side } 1} 1$ & 2 & 3 & 4 & 5 & 6 & 7 & $\frac{\text { Side } 2}{8}$ & $\begin{array}{c}\text { Average } \\
\text { ("Mean") }\end{array}$ & $\begin{array}{c}\text { Standard } \\
\text { Error } \\
\varepsilon^{\mathrm{a}} \\
\end{array}$ & $\begin{array}{c}\text { Standard } \\
\text { Deviation } \\
s^{\mathrm{a}} \\
\end{array}$ & Date \\
\hline Top & 60 & 77 & 74 & 77 & 80 & 75 & 81 & 83 & 75.9 & 2.3485 & 7.59 & $9-26-08$ \\
\hline Center & 74 & 76 & 73 & 73 & 79 & 78 & 79 & 79 & 76.4 & 0.91 & 2.94 & $9-26-07$ \\
\hline Clip End & 74 & 70 & 74 & 80 & 78 & 77 & 80 & 80 & 76.5 & 1.1859 & 3.83 & $9-26-07$ \\
\hline
\end{tabular}

a. Standard Error $\varepsilon$ was reported by ConfiDent software, and used here to calculate the Standard Deviation, $s$.

Table E-7. Vickers Hardness $\mathrm{H}_{\mathrm{V}}$ measurements across aluminum in DU10Mo sample fabricated with Anviloy FB tool face.

\begin{tabular}{|c|c|c|c|c|c|c|c|c|c|c|c|c|}
\hline Location & $\underline{\text { Side } 1}$ & 2 & 3 & 4 & 5 & 6 & 7 & $\frac{\text { Side } 2}{8}$ & $\begin{array}{l}\text { Average } \\
\text { ("Mean") }\end{array}$ & $\begin{array}{c}\text { Standard } \\
\text { Error } \\
\varepsilon^{\mathrm{a}} \\
\end{array}$ & $\begin{array}{c}\text { Standard } \\
\text { Deviation } \\
s^{\mathrm{a}} \\
\end{array}$ & Date \\
\hline Top & 77 & 77 & 80 & 80 & 78 & 80 & 84 & 80 & 79.5 & 0.7706 & 2.49 & $10-1-08$ \\
\hline Center & 73 & 73 & 77 & 75 & 78 & 78 & 80 & 84 & 77.2 & 1.2247 & 3.96 & $10-1-07$ \\
\hline Clip End & 75 & 75 & 73 & 76 & 73 & 71 & 74 & 71 & 73.5 & 0.6374 & 2.06 & $10-1-08$ \\
\hline
\end{tabular}

a. Standard Error $\varepsilon$ was reported by ConfiDent software, and used here to calculate the Standard Deviation, $s$. 
APPENDIX E - Friction Bonding Sample Hardness Measurements with Statistics

Table E-8. Vickers Hardness $\mathrm{H}_{\mathrm{V}}$ measurements along foils in DU10Mo samples fabricated with varying FB tool face alloys.

\begin{tabular}{|c|c|c|c|c|c|c|c|c|c|c|}
\hline $\begin{array}{l}\text { Sample FB } \\
\text { with Tool } \\
\text { Face Alloy }\end{array}$ & $\frac{\text { Top }}{1}$ & 2 & 3 & $\frac{\text { Center }}{4}$ & 5 & 6 & $\frac{\text { Clip End }}{7}$ & $\begin{array}{c}\text { Average } \\
\text { ("Mean") }\end{array}$ & $\begin{array}{c}\text { Standard } \\
\text { Deviation } \\
s^{\mathrm{a}} \\
\end{array}$ & Date \\
\hline Steel-A36 & 289 & 276 & 286 & 292 & 286 & 284 & 295 & 287 & 6.12 & 10-3-07 \\
\hline Al-Bronze & 282 & 286 & 288 & 286 & 264 & 262 & 258 & 275 & 13.2 & 9-26-07 \\
\hline Anviloy & 290 & 290 & 284 & 288 & 284 & 282 & 279 & 285 & 4.19 & $10-3-07$ \\
\hline
\end{tabular}

